\title{
WestVirginiaUniversity
}

THE RESEARCH REPOSITORY @ WVU

Graduate Theses, Dissertations, and Problem Reports

2000

\section{Encoding of periodic skin stimuli by spinal dorsal horn neurons}

Jeffrey J. Lawson

West Virginia University

Follow this and additional works at: https://researchrepository.wvu.edu/etd

\section{Recommended Citation}

Lawson, Jeffrey J., "Encoding of periodic skin stimuli by spinal dorsal horn neurons" (2000). Graduate Theses, Dissertations, and Problem Reports. 1210.

https://researchrepository.wvu.edu/etd/1210

This Dissertation is protected by copyright and/or related rights. It has been brought to you by the The Research Repository @ WVU with permission from the rights-holder(s). You are free to use this Dissertation in any way that is permitted by the copyright and related rights legislation that applies to your use. For other uses you must obtain permission from the rights-holder(s) directly, unless additional rights are indicated by a Creative Commons license in the record and/ or on the work itself. This Dissertation has been accepted for inclusion in WVU Graduate Theses, Dissertations, and Problem Reports collection by an authorized administrator of The Research Repository @ WVU.

For more information, please contact researchrepository@mail.wvu.edu. 


\title{
Encoding of Periodic Skin Stimuli by Spinal Dorsal Horn Neurons
}

\author{
by \\ Jeffrey J. Lawson \\ Dissertation submitted to the School of Medicine \\ at West Virginia University \\ in partial fulfillment of the requirements \\ for the degree of \\ Doctor of Philosophy \\ in \\ Medical Physiology
}

\author{
Approved by \\ Paul Brown, Ph.D., Committee Chair \\ James Culberson, Ph.D. \\ J. Vernon Odum, Ph.D. \\ H. Richard Koerber, Ph.D. \\ Ronald Millecchia, Ph.D. \\ Department of Physiology \\ Morgantown, West Virginia \\ 2000
}

Keywords: dorsal horn, cutaneous, periodic, neuron Copyright 2000 Jeffrey J. Lawson 


\section{ABSTRACT \\ Encoding of Periodic Skin Stimuli by Spinal Dorsal Horn Neurons Jeffrey J. Lawson}

The encoding of vibrotactile pitch by neurons of the somatosensory system has been investigated at many levels of the neuraxis. These studies, however, have not extended to the spinal dorsal horn, which is known to process low threshold mechanoreceptive input. The studies presented here were designed to assess the possible role of the dorsal horn in the encoding of vibrotactile pitch. To accomplish this task, dorsal horn single unit extracellular recordings were obtained during periodic electrical and sinusoidal mechanical stimulation of their receptive fields. Techniques used in classifying vibrotactile pitch encoding were similar to those used in other somatosensory nuclei, including analyses of firing rate and periodicity. Firing rate profiles, phase vectors, histograms and power spectra were used to quantify the responses. The analyses indicate that for most cells, firing rate is adequate to encode stimuli at $50 \mathrm{~Hz}$ and below, but insufficient at 100 and $200 \mathrm{~Hz}$. A small number of neurons were able to encode stimulus frequencies of $50 \mathrm{~Hz}$ and higher in their firing rates. Periodicity is present in most cell discharges at all frequencies. This leads to the conclusion that at least some dorsal horn neurons are able to encode vibrotactile pitch in a way similar to that found in other somatosensory areas, at least with regard to periodicity and firing rate. 


\section{Table of Contents}

\section{TOPIC}

PAGE

Abstract........................................................................

Table of Contents.......................................................... iv

List of Figures.................................................................................. vi

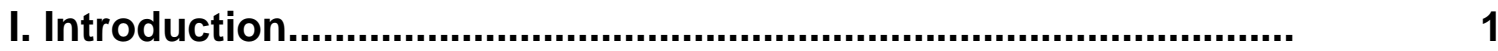

II. Review of Literature....................................................................

A. Psychophysics of vibrotaction..........................................

B. Neurophysiology: study of peripheral receptor

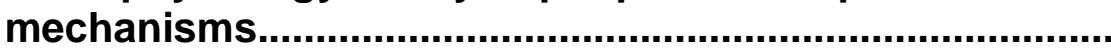

C. Neurophysiology: Studies of subcortical frequency following.

D. Neurophysiology: Studies of cortical neuronal responses.

III. Methods

A. Project I: Transdermal electrical stimulation of DHC RFs.

1. Surgical Procedures....................................................

2. Transdermal stimulation of DHC RFs...................... 23

3. Recording of responses..........................................

4. Analysis of data obtained from electrical stimulation of DHCs.

a. Histogram generation..................................... 26

b. Analysis of interval files..................................

c. Vector strength analyses............................... 28 
d. Power spectra................................................. 30

e. Measures of firing rates in cell discharges.... 31

f. Adaptation......................................................... 31

g. Population response to electrical stimuli....... 32

B. Project II: Responses of DHCs to sinusoidal mechanical stimuli............................................ $\quad 34$

1. Surgical procedures...................................................

2. Sinusoidal cutaneous stimulation............................ 34

3. Recording of responses............................................. 36

4. Data analysis methods...........................................

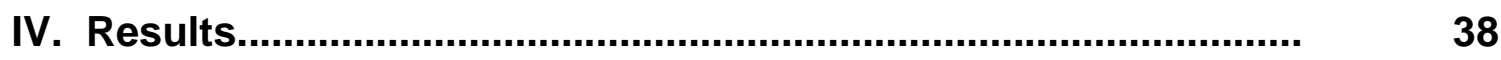

A. Electrical stimulation of DHC RFs...................................... 39

1. Histogram analysis............................................... $\quad 39$

2. Vector strength analysis.........................................

3. Analysis of power spectra.......................................... 48

4. Analysis of firing rates..........................................

5. Population responses............................................ 61

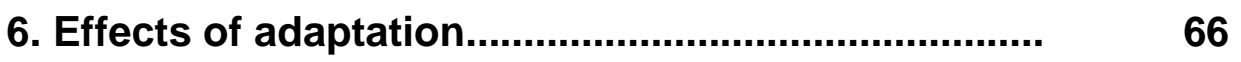

B. Mechanical stimulation of DHC RFs................................... $\quad 70$

1. Histogram analysis............................................... 70

2. Vector strength analysis.........................................

3. Analysis of power spectra........................................ 88

4. Analysis of firing rates........................................... 
5. Population responses................................................ 103

6. Effects of adaptation................................................ 108

VI. Discussion................................................................................

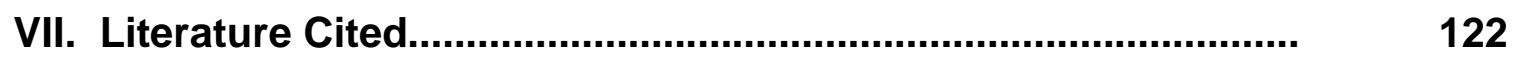




\section{List of Figures}

FIGURE 1. Stimulation paradigm for delivery of electrical stimuli... 26

FIGURE 2. Generation of phase vectors............................ 31

FIGURE 3. Mechanical stimulation paradigm....................... 39

FIGURE 4. RFs of DHCs characterized electrically ................. 42

FIGURES 5-8 PSTHs of 3 DHCs stimulated electrically at four stimulation rates................................... 43-46

FIGURE 9. Phase vectors of DHC responses during four rates of Electrical stimulation.................................... 48

FIGURE 10. Summary histograms of phase vectors during four rates of electrical stimulation.

FIGURE 11. Mean phase vector strengths at four rates of electrical Stimulation.......................................................... 50

FIGURE 12. Rao's spacing test for phase vectors................... 51

FIGURE 13. Autocorrelograms of responses to 4 rates of electrical Stimulation.............................................. 53

FIGURE 14. Phase histograms of cells in fig. $13 \ldots \ldots \ldots \ldots \ldots \ldots \ldots . \ldots . \ldots . \ldots 54$

FIGURE 15. Power spectra of one cell at four stimulation rates..... 55

FIGURE 16. Principal frequency components of responses at four stimulation rates...................................................... 56 
FIGURE 17. Coefficients of variation for principal frequency components at four rates of stimulation.

FIGURE 18, Summary histograms for firing rates......................... 58

FIGURE 19. Firing rates of all cells at four rates of stimulation....... 59

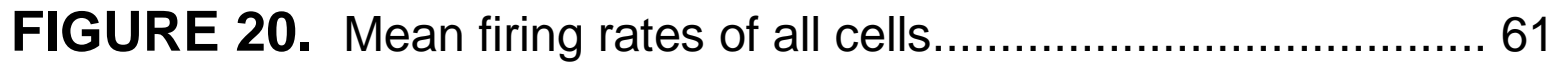

FIGURE 21. Percentage of cells with bursting activity.................... 62

FIGURE 22. Relationship between firing rate and vector strength at four stimulation rates............................................ 63

FIGURE 23. DHC population autocorrelograms for responses at four stimulation rates............................................... 64

FIGURE 24. Power spectra for population responses..................... 65

FIGURE 25. Phase vectors for responses to ten stimulus pulses... 68

FIGURE 26. Vector strength summary histograms for responses to ten stimulus pulses.............................................. 69

FIGURE 27. Mean vector strengths for responses to ten stimuli... 70

FIGURE 28. Firing rate summary histograms, ten stimuli................ 71

FIGURE 29. Mean firing rates during ten stimuli............................ 72

FIGURE 30. RF locations in mechanical study............................ 73

FIGURES 31-35. PSTHs of three DHC responses to 5 frequencies of sinusoidal stimulation at four amplitudes........... 75-79 
FIGURES 36-40. Phase vector diagrams for DHC responses to 5 frequencies of stimulation at four amplitudes.... 80-84

FIGURES 41-45. Vector strength summary histograms for DHC responses to 5 frequencies of stimulation... 85-89

FIGURE 46. Vector strength vs. amplitude at 5 stimulus frequencies. 90

FIGURE 47. Vector strength vs. frequency at four stimulus amplitudes 91

FIGURES 48-52. Principal frequency components of power spectra for 5 frequencies of stimulation at four amplitudes $92-96$

FIGURES 53-57. Summary firing rate histograms for 5 frequencies of stimulation at four amplitudes 98-102

FIGURE 58. Firing rate vs. amplitude at five frequencies of frequencies of stimulation 103

FIGURE 59. Firing rate vs. frequency at four amplitudes of stimulation. 104

FIGURE 60. Mean firing rates at maximal amplitude for five frequencies of stimulation 105

FIGURES 61-62. Population autocorrelograms for 5 frequencies of stimulation at maximal amplitude.................. 107-108

FIGURES 63-64. Population power spectra for 5 frequencies of stimulation at maximal amplitude. $109-110$

FIGURE 65. Effect of increasing amplitude on firing rate during 10 cycles of stimulation at four frequencies. 
FIGURE 66. Effect of increasing amplitude on vector strength during 10 cycles of stimulation at five frequencies.. 112

FIGURE 67. Effect of increasing frequency on vector strength during 10 cycles of stimulation at four amplitudes.. 113

FIGURE 68. Effect of increasing frequency on firing rate during 10 cycles of stimulation at four amplitudes.............. 114 


\section{Introduction}

One of the central issues in sensory neurobiology is the elucidation of candidate neural codes that can be used by an organism to perform discriminations. Specifically, patterns of action potentials are examined in single neurons or groups of neurons, and hypotheses are made regarding the information contained in discharge patterns in response to a controlled stimulus. The neurons that are studied must be candidates to contribute to the pathways carrying information about the sensation to be examined. If possible, it must be demonstrated that the hypothesized criterion information carried in a spike train changes in a systematized way to a changing stimulus. That information, when combined with psychophysical experiments, can lead to a description of how the nervous system encodes a sensory event, and how those codes are used to perform sensory discriminations.

The somatosensory system is an ideal system in which to study sensory coding. The organization of its components have been well studied, and many previous studies have examined spatial and temporal coding of skin stimuli by individual neurons. Many studies have examined encoding at all levels of the somatosensory neuraxis, including peripheral receptors, spinal cord, dorsal column nuclei, thalamus, and several areas of the somatosensory cortex. The studies can be broken down into two main classes: encoding of spatially varying stimuli, and encoding of temporally varying stimuli.

The responses of somatosensory neuronal elements to skin vibration have been studied in primary afferents, dorsal column nuclei, thalamus and cortex. The first three of those areas include elements that convey vibration information to the brain. 
Interestingly, there is one potential pathway missing from previous experiments. Cells of the spinal dorsal horn are known to convey information to higher levels of the somatosensory system, through a number of ascending tracts. It seems reasonable to ask if this system is capable of producing candidate neural codes for encoding vibrotactile pitch in the same way that other ascending systems (e.g. dorsal column/medial lemniscal system) do. Spinal dorsal horn cells, like those of the dorsal columns and the trigeminal sensory nuclei, receive primary afferent input directly, so it is possible that these cells respond in a similar fashion to those in similar nuclei.

One possibility for the neural coding of vibration is frequency following. That is, the temporal discharge pattern of a sensory neuron is directly related to the frequency of vibration. This can be manifested as a one-to-one following (one spike per vibration cycle), or it can be more subtle, as in a phase locked response. Both of these types of coding have been demonstrated in other areas of the somatosensory system.

Alternatively, neurons may respond in an aperiodic fashion to periodic stimuli and still convey information about vibration frequency if they are frequency tuned, i.e. they respond over a restricted range of stimulus frequencies. The auditory system contains neurons of this type. This possibility is more difficult to assess and has never been studied in the somatosensory system.

It is the purpose of this research to determine if there are neurons present in the spinal dorsal horn that are able to encode vibratory information by means of firing rate and periodicity. 


\section{REVIEW OF LITERATURE}

The study of vibrotaction has proceeded along several lines in the past century, and numerous experiments have been performed in the areas of psychophysics, anatomy and neurophysiology. As research methods become more sophisticated, we are moving closer to the ability to correlate aspects of consciousness with neuronal events.

\section{A. Psychophysics of vibrotaction}

Many vibratory psychophysical experiments have been carried out in both humans and non-human primates. One thing the human studies seem to agree on is that there are different psychophysical 'channels' responsible for carrying information to consciousness. Each of these channels carries a particular portion of the vibration frequency spectrum. Verillo's group has concluded that in humans there are four psychophysical channels: one P (Pacinian) channel and three non-Pacinian (NPI, NPII, and NPIII) channels in glabrous skin (Bolanowski et al., 1988). The Pacinian channel, named after the receptor type (Pacinian corpuscle), handles the highest frequency vibrations, and the NPI, NPII and NPIII handle vibration outside the Pacinian frequency range $(<70 \mathrm{~Hz})$. The NP channels can be separated under various masking conditions. The perception of vibration is also temperature sensitive (Verrillo et al. 1985). In hairy skin, there appear to be 3 channels, one Pacinian and two non-Pacinian (Bolanowski et al. 1994). There is the possibility that discrete groups of neurons could carry 
information from each pathway, but this it not necessary for the pathways to exist. As long as the information reaches the correct neurons in the cortex, the can reach their destination via many different routes.

Microneurography has also shed some light on the percepts elicited by stimulation of cutaneous receptors. These experiments allowed for recording and stimulation of single afferent fibers in peripheral nerves. Studies by Torebjork (Torebjork et al. 1980; Torebjork et al. 1984; Torebjork 1987) and Vallbo (Vallbo and Johansson, 1984) showed that stimulation of the receptive field (RF) of a single cutaneous afferent was sufficient to elicit a percept in test subjects. Slowly adapting type I (SAI) RF stimulation produced a sensation of pressure, rapidly adapting (RA) stimulation produced a sensation of flutter, and Pacinian corpuscle (PC) stimulation produced a sensation of vibration. Slowly adapting type II (SAII) RF stimulation failed to cause a percept in test subjects. Interestingly, these experimenters were also able to stimulate the fibers electrically, and they found that increasing frequency of stimulation of the fibers caused an increasing sense of pressure for SAl fibers, an increased tapping or flutter frequency in RA receptors, and increasing vibrational frequency in PC fibers. These results suggest that in humans the RA and PC fibers are responsible for the sensations of flutter and vibration, as originally defined by Mountcastle (Mountcastle et al. 1967).

Many other groups have done psychophysical testing in humans, and Mountcastle's group did comparative studies between monkeys and humans (Mountcastle et al., 1990). These were performed to show the similarity of vibration discrimination ability in humans and neurophysiology gathered in nonhuman primates. 
Their psychophysical findings showed that vibratory discriminability was very similar for monkeys and humans, and this served as the groundwork for their and others' studies of the neural correlates of vibrotaction through examination of central and peripheral neuronal periodicities. Most of the psychophysical testing of vibrotactile ability has been done in primates, although much of the neurophysiological information has been gathered in other species.

Cohen et al. (1999) recently looked at the relationship between sinusoidally driven stimulus probes and the skin surface to show the degree of coupling between the two. This group used stroboscopic illumination and video microscopy to verify the relationship between the skin and probe tip. At amplitudes of $.25 \mathrm{~mm}$ and frequencies up to $100 \mathrm{~Hz}$, they found that no significant decoupling occurred, and they conclude that sinusoids of these frequencies and amplitudes are appropriate for testing.

Lloyd et al. (1999) looked at the effects of cross-modal directed attention on performance of a vibration detection task at $100 \mathrm{~Hz}$. When human subjects focus visually on a body part that is receiving skin stimulation, the perception of vibration appears to be facilitated.

\section{B. Neurophysiology: studies of peripheral receptor}

\section{mechanisms}

The response characteristics of low threshold cutaneous mechanoreceptors have been determined in many different species, including humans (using the microneurography technique), under different stimulus control paradigms. Most of the 
studies of cutaneous mechanoreceptors are in agreement across species with regard to the submodality classification of each, even if the nomenclature has differed from laboratory to laboratory. For the purposes of this review, only mammalian mechanoreceptors will be discussed.

While specific mechanoreceptor responses to stimuli are somewhat conflicting in the literature, a broad classification scheme exists. Receptors have been studied in rat (Fitzgerald 1987), raccoon (Pubols 1983), rabbit (Brown and lggo, 1967; Brown and Hayden 1971), cat (e.g. Burgess at al. 1968; Burgess et al. 1974; Chambers et al. 1972; Horch et al. 1977; Iggo and Ogawa 1977; Janig 1971a and 1971b; Whitehorn 1973; Tapper 1972), monkey (Lindblom, 1965; Harrington and Merzenich, 1970; Johnson, 1974; Talbot et al. 1968; Werner 1965) and human (Cohen and Vierck 1993a and 1993b; Hulliger et al. 1979; Johansson and Vallbo 1979; Knibestol 1973; Knibestol 1975; Vallbo and Hagbarth, 1968). All of these studies taken together have shown the relationships between anatomy, physiology and percept for each receptor. SAI receptors are Merkel cells and respond to pressure. SAll receptors are Ruffini endings and respond to skin stretch, usually in a preferred direction. RA receptors are Meissner's corpuscles and respond to flutter. PCs are named after their receptor structure, and respond to vibration. There are three types of hair receptors, G1 (tylotrich), G2 (guard) and D (down) hair. The field receptor (F) responds to skin stretch. All low threshold receptor types are innervated by $A \alpha$ or $A \beta$ myelinated axons, except D-hair receptors, which are innervated by $A \delta$ axons.

The responses of the SAI and SAll receptors are a sustained discharge to a constant applied pressure to its RF. RA and hair follicle receptors show a phasic 
response to an applied stimulus. Subcutaneous Pacinian receptors' lamellated structures serve as high pass filters for cutaneous vibration. Many Pacinian receptors are also found in deeper tissues (e.g. mesentery, Pacinian corpuscle function and anatomy reviewed in Bell, 1994). The details of the frequency following characteristics to vibration vary from study to study, but the convention put forth by the Mountcastle group (Talbot et al.1968) has become the standard by which receptors' roles in transmitting different parts of the vibratory spectrum have been classified. This classification has been used not only at the receptor level, but at higher levels of the somatosensory system as well. In this scheme, SA receptors respond to vibratory stimuli of low frequencies, less than about $20 \mathrm{~Hz}$. RA receptors transduce vibrational stimuli from 20 to approximately $80 \mathrm{~Hz}$ ('flutter'), and PC receptors transduce vibrational frequencies above $80 \mathrm{~Hz}$ to at least $250 \mathrm{~Hz}$. These classifications describe what part of the vibrotactile spectrum each receptor responds to best. Some exceptions to this scheme are apparent. The tuning curves of Tapper et al. (1972) showed that SAI receptors can follow vibrational frequencies well in excess of $200 \mathrm{~Hz}$. Gynther et al. (1992) found that SAll receptors respond to low amplitude $(<100 \mu)$ vibration with a tightly phase-locked, regular 1:1 discharge over a bandwidth that extends to $600 \mathrm{~Hz}$, and responses remained phase-locked up to $1000 \mathrm{~Hz}$. The receptors most likely to be responsible for transmission of all but the lowest vibratory frequencies would be the RAs and PCs (from combined psychophysical and receptor data), but other receptor types are able to transduce vibration as well. The apparent discrepancy between studies could be caused by different pre-indentations of the stimuli, size of the stimuli, or even species differences. 


\section{Neurophysiology: studies of subcortical frequency}

\section{following}

Since the studies of mechanoreceptors showed that there are at least some types of receptors in the skin that could preserve frequency of vibration in their afferents' firing patterns, neurophysiologists looked to central nervous system cells to see if these temporal patterns were preserved at the postsynaptic level. The somatosensory nuclei that process somesthetic information include, but are not limited to, the spinal dorsal horn, the dorsal column nuclei, the lateral cervical nucleus (most extensively developed in carnivores), the sensory trigeminal nucleus, the thalamus and the somatosensory cortex (areas SI and SII). The cortex and the dorsal column nuclei have been studied in the greatest detail, and the spinal cord in the least. The sentiment expressed in most of the literature is that the dorsal column pathway is dominant in transmission of temporally patterned vibrotactile information. This is probably because a large amount of information is known about the dorsal column-medial lemniscal system, where much is known about the transmission of vibrotactile information.

The dorsal column/medial lemniscal pathway is by far the most extensively studied with regard to vibrotactile information transfer. The studies of this ascending system fall into two broad categories: studies of single unit discharges in the dorsal column nuclei, and psychophysical studies demonstrating functional deficits apparent in animals and humans with dorsal column pathway lesions. 
Rowe's group has collected a large amount of single unit dorsal column nuclei data. They have found that cells of the dorsal column nuclei are able to follow vibratory stimuli at frequencies up to $200 \mathrm{~Hz}$ (Ferrington 1987). Their measure of frequency following capacity was percent entrainment, in which the half-cycle of the sinusoid containing the largest number of responses (from a phase histogram) was divided by the total number of responses and multiplied by $100 \%$. They classified gracile and cuneate nucleus cells by using peripheral receptor terminology, defining cells as having slowly adapting, rapidly adapting, or Pacinian response profiles. The implication from that classification scheme is that there are submodality specific neurons in the dorsal column nuclei that probably receive only one type of functionally dominant afferent input. This information was used to reinforce the labeled line idea of segregation of somatosensory submodalities in the ascending pathways. One reason this classification was done was because submodality specific cortical cells had been described decades earlier (e.g. Talbot et al. 1968).

Another interesting aspect of the dorsal column pathway that Rowe and colleagues found was that there is a high degree of synaptic security between PC afferents and dorsal column nucleus cells. Ferrington et al. (1987b) studied the relationship between firing patterns in Pacinian afferents and discharges in postsynaptic dorsal column nucleus cells. When one Pacinian fiber was activated, the cell it was connected to responded in a phase-locked fashion, and there was a one-to-one response to input for frequencies from $200-400 \mathrm{~Hz}$. Phase locking was observed at $500 \mathrm{~Hz}$ as well. This led this group to conclude that dorsal column nucleus neurons are capable of transmitting information in a secure fashion from inputs as small as one 
peripheral afferent's discharge. To further examine this idea, they studied dorsal column nucleus neurons and stimulated two Pacinian afferents sequentially (Ferrington, 1987c). They stimulated one Pacinian afferent and recorded the postsynaptic response, then introduced a second Pacinian input at the same frequency. The cell remained phase-locked, as one would expect. They then changed the phase and frequency of the second input to see what would happen in the temporal discharge of the postsynaptic neuron. Their conclusion was that a dorsal column nucleus cell tends to phase lock to its first input, and even the introduction of a second antagonistic input doesn't change its discharge pattern. Thus, dorsal column nuclei cells are probably driven by just a few primary afferents so that temporal integrity of vibrotactile frequency information can be maintained. This research showed that relay nuclei in the dorsal column/medial lemniscal pathway were able to preserve the firing patterns of primary afferents to vibration.

Rowe's group also looked at the effects of stimulating other receptor types on the firing patterns of dorsal column nucleus cells. Vickery et al. (1994) looked at the response of cuneate cells to vibratory stimulation of single SAI receptors. They monitored a peripheral nerve to show they were stimulating only one SAI, and they found that SAl fibers could follow vibration up to $500 \mathrm{~Hz}$ in a 1:1 fashion. The cuneate cells they were connected to could only follow in a 1:1 fashion out to approximately 150 $\mathrm{Hz}$. They concluded that the transmission characteristics of SAI-cuneate cell pairs are as secure as Pacinian-cuneate cell pairs. Slowly adapting type II fibers were examined in the same way (Gynther et al., 1995). The conclusions from the studies of these 
relationships is that security of synaptic transmission in the dorsal column nuclei is very tight.

Insight into the function of the dorsal column pathway has also been gained through the study of perceptual deficits of animals and humans who have some sort of dorsal column system disruption. Vierck (1974) showed that the dorsal columns were not necessary for spatial localization, as this perceptual ability was unaffected by dorsal column section. This group then turned their attention to the necessity of the dorsal column pathway in temporal discrimination. They studied the ability of monkeys to do vibratory discriminations of stimuli at 10 and 35 pulses per second (non-sinusoidal) (Vierck et al. 1985) following disruption of the ipsilateral dorsal columns, contralateral anterolateral column, and ipsilateral dorsolateral column. The lesions to the dorsolateral and anterolateral columns produced no deficits in vibratory discrimination following recovery, but the dorsal column section did affect discrimination. This group concluded that the ipsilateral dorsal columns are necessary for the discrimination paradigm they were testing. It is interesting to note that neither of these frequencies of stimulation falls into the submodality of vibration, but actually are in the domain of flutter, which presumably is carried by rapidly adapting afferents, not Pacinians. Vierck's group also studied the evoked potentials from spinal pathways, and found that the anterolateral column and dorsolateral column potentials were attenuated more than the dorsal column evoked potential at 50 pulses per second stimulation (relative to the potential recorded with 1.5 pps stimulation) (Makous et al. 1996). In that same study, they looked at the amplitude of cortical evoked potentials in response to a $10 \mathrm{pps}$ stimulus, and found that there was a less pronounced response in dorsal column 
sectioned animals than there was in the same animals before lesion. The authors concluded that the dorsal columns were important for information transfer at this frequency.

Other studies have focused on trying to describe the importance of the dorsal columns with regard to other perceptual events. It has been found that the dorsal columns are important in tasks which require working memory for serial order acts (Dubrovsky et al. 1971). There is also an implication for the importance of the dorsal columns in kinesthesia and proprioception, such as that seen in a finger movement task (Cooper et al. 1993). In summary, the established theory is that the dorsal column/medial lemniscal pathway is the part of the ascending somatosensory system responsible for transmission of temporal skin information.

One very rare human clinical case has shed some light on the function of ascending somatosensory pathways. In this case, first reported by Noordenbos and Wall (1976), a woman suffered a catastrophic spinal lesion from a stab wound to the back, transecting all but one anterolateral quadrant at the level of the third thoracic vertebra. The experimenters used this case as an opportunity to study the information that was still able to reach the woman's consciousness through the remaining white matter. They tested several submodalities, including temperature, pinprick, localization of pressure and touch, passive movement detection, and vibration (100 Hz tuning fork). Amazingly, most of the tests came out nearly normal, with the exception of vibration, in which the patient was found to be unresponsive. An electrical stimulus of 10 and 100 $\mathrm{Hz}$ was used for frequency discrimination. The patient was unable to perform the discrimination on either side, and was unable to detect a $50 \mathrm{~Hz}$ mechanical sinusoid as 
well. These results show that, in this patient, the anterolateral quadrant (which contains the spinothalamic tract) is at least sufficient to subserve the submodalities tested, with the exception of vibration. There was a follow-up on this patient 20 years later (Danziger et al. 1996), and there were a few long term plastic changes, as assessed with MRI and PET scans, as well as psychophysical testing. Interestingly, vibration sense was the only submodality that appeared to have improved at all in that time. Other psychophysical testing has been done on human patients (e.g. Nathan et al. 1986). The conclusions of those studies were based on patients who had lesions of various tracts due to disease or surgical treatment. Unfortunately, the exact extent of about half of the lesions was never verified postmortem, making interpretation of function of partially disrupted ascending pathways very difficult, if not impossible.

The thalamus has also been studied with regard to following of temporal somatosensory stimuli. Poggio and Mountcastle (1963) and Mountcastle et al. (1964) examined the characteristics of cell firing patterns in the VPL nucleus of the primate thalamus, and this work was followed up many years later by Ghosh et al. (1992). These studies showed that there are thalamic neurons that are able to preserve frequencies of stimulation well within the range of vibration $(>200 \mathrm{~Hz})$ in their discharge patterns. This is not surprising, since in cat it has been shown that the thalamus serves as a relay for Pacinian input to the cortex (Herron and Dykes 1986). Neurons in the thalamus show response characteristics similar to those in the dorsal column nuclei, even though they are one synapse further away from the periphery.

The responses of trigeminal sensory cells to vibration have been examined, but not in nearly the exhaustive manner of the thalamus, dorsal column nuclei, or cortex. 
Mosso and Kruger (1973) and Kirkpatrick and Kruger (1975) looked qualitatively at the response characteristics of cells in the trigeminal sensory nucleus, and came to the conclusion that the different 'receptor types' were represented in this sensory nucleus, i.e. SA, RA, and PC. Oscilloscope traces of responses are shown, and the authors report 'entrainment of one or more impulses per cycle was observed up to $700 \mathrm{~Hz}$ '. Donevan and Abrahams (1993) examined cat trigeminal neurons with RF in the planum nasale, and they found that neurons could be entrained at frequencies below $80 \mathrm{~Hz}$. They concluded that the upper frequency limit was due to the absence of the Pacinian corpuscles in the planum nasale. They also found slowly adapting neurons in this area. The trigeminal sensory nucleus is another example of a somatosensory nucleus that contains neurons capable of encoding vibrotactile pitch.

Studies of the role of the spinal cord transferring vibrotactile information are much less prevalent than studies conducted in other somatosensory nuclei and the periphery. Oswaldo-Cruz and Kidd (1964) stimulated RFs of lateral cervical nucleus cells electrically, and showed that responses declined starting at $50 \mathrm{~Hz}$. A.G. Brown's group $(1969,1987)$ studied the responses of spinocervical tract cells, and found cells that responded to light touch, although responses to systematized vibration was not studied. P. B. Brown (1969) looked quantitatively at the response of dorsal horn cells to variations in intensity, area and location of stimuli within their RFs. The effects of vibration were not studied, and in fact cells that were encountered that could follow $100 \mathrm{~Hz}$ dorsal column volleys were excluded from analysis. Salter and Henry (1990) looked at spinal wide dynamic range cells, and found that firing rates in these cells were attenuated by 120 and $240 \mathrm{~Hz}$ vibration, although the temporal pattern of the responses 
was not analyzed. De Konnick and Henry (1992) followed up that study by looking at nociceptive dorsal horn cells, and found that vibration of $80-250 \mathrm{~Hz}$ caused a hyperpolarization in these cells. Edgley and Jankowska (1988) looked at dorsal spinocerebellar tract cells, and found that they could be monosynaptically excited by stimulation of cutaneous receptors, but no specific reference is made to vibration. One interesting finding about dorsal horn cells is that there are no reports of cells that have 'receptor-like' qualities to their responses, i. e. no RA or PC dorsal horn cells. In fact, Tapper et al. (1973) reported that there is a submodality convergence onto dorsal horn cells.

\section{Neurophysiology: studies of cortical neuronal responses}

The cortex has been examined more thoroughly than any other component of the somatosensory system with regard to the study of neuronal responses to vibratory stimuli. Following their studies of frequency following characteristics in primary afferents (Talbot 1968), the Mountcastle group looked at monkey somatosensory cortex to see if there are comparable discharge patterns in postsynaptic elements (Mountcastle et al. 1969; Mountcastle et al. 1990a; Mountcastle 1990b). Their intent was to show that the psychophysics obtained in humans and monkeys are adequately described by the periodicities of firing of SI neurons. These recordings were made while the monkeys were performing a vibration discrimination task, so there was an attentive component present. Monkey and human vibrational discrimination were compared, and the sensibility of the two primate species was found to be very similar. 
SA and RA neurons were studied, and their periodicities measured via harmonic analysis and construction of autocorrelograms. Their conclusion was that overall firing frequency couldn't be the discriminandum, since easily discriminated frequencies produce no statistically significant changes in firing frequency. However, the discrimination is based on a system in which the same sets of neurons in the postcentral gyrus receive two applications of a vibratory stimulus, each eliciting an ensemble firing pattern. To make a discrimination, the animal must compare a second stimulus with one preserved in memory. The explanation is that the representations of the two frequencies differ in period length sufficiently to arrive at a 'different' judgment. Since memory is involved as well as the neurons being studied, the key element of the cortical cell discharge pattern used for discrimination is hard to determine. There definitely is entrainment in cortical neurons in this study, at least in the range of flutter.

There have been other studies of cortical responses to skin vibration showing that elements of the somatosensory cortices are able to entrain to higher frequencies. Rowe's group (Bennett et al. 1980) studied SII neurons and classified their responses in a paradigm that was similar to their work in the dorsal column nuclei. They found different receptor type submodality classifications in these cortical neurons, although the distribution of types seemed different. Burton and Sinclair (1991) studied the response of monkey SII cells to vibration ranging from 10 to $300 \mathrm{~Hz}$. They found that most cells were unable to follow frequencies of greater than $10 \mathrm{~Hz}$, although they found smaller subpopulations of cells that responded well to different parts of the vibrotactile spectrum: $50-75 \mathrm{~Hz}$ and over $100 \mathrm{~Hz}$. Cells that followed $50-75 \mathrm{~Hz}$ displayed a precisely time locked firing pattern, and cells that responded to $>100 \mathrm{~Hz}$ had decreased probability 
of firing with successive stimulus cycles. The cells that responded to $>100 \mathrm{~Hz}$ also preferentially responded to low amplitudes, leading the authors to conclude that SII may be involved in processing vibrotactile information from Pacinian afferents.

Lebedev and Nelson (1996) recorded monkey SI cortical neurons during a vibration-cued wrist flexion task. Vibration was applied at 57 and $127 \mathrm{~Hz}$, and neuronal responses quantified. Neurons were found that entrained well at $127 \mathrm{~Hz}$, although they constituted only $5 \%$ of the total sample. These high frequency vibratory sensitive neurons appeared to be equally distributed across $3 a, 3 b, 1$ and 2 (with the fewest number coming from 3a). The authors concluded that there are cells that receive Pacinian afferent input in SI. Response latencies for neurons in different areas varied, implying hierarchical processing of vibratory input.

The ability of SI and SII to modulate each others' responses was studied by Rowe's group (Turman et al. 1995; Zhang et al 1996). In these studies, coolinginduced reversible deactivation of SI and SII were used to study SII and SI, respectively. They concluded that SI appears to receive its information directly from thalamocortical sources, and its responses are affected little by SII deactivation. A like result was found for the effect of SI deactivation on SII responses, suggesting that the two cortical areas receive independent projections.

Tommerdahl et al. (1996) used 2-deoxyglucose uptake to study the cortical response to $10-25 \mathrm{~Hz}$ brushing stimuli, and the effects of dorsal column section on that response in monkeys. They used the ipsilateral hemisphere as a control during the lesions, and found a decreased response profile in animals following dorsal column section. Tommerdahl et al. (1998) also used an imaging system (Optical Intrinsic 
Signal) to look at distribution of cortical responses to $25 \mathrm{~Hz}$ flutter. Changes in reflectance were found to be strongest in areas $3 b$ and 1 . Another study of mass action in the cortex involved the use of temporal analysis of EEG patterns elicited by vibration (Kelly et al. 1997). They found 'driving' frequencies at the frequency of stimulation by using frequency analysis techniques. Another study by this group (Tommerdahl et al. 1999) looked at the distribution of OIS signal for same site skin flutter $(25 \mathrm{~Hz})$ vs. vibration $(200 \mathrm{~Hz})$ to determine if different cortical areas are excited by different frequencies of skin displacement. Their findings show that there is an increase in absorbance (measure of the OIS signal) in contralateral SI and SII to $25 \mathrm{~Hz}$ central footpad stimulation in cats. The response to $200 \mathrm{~Hz}$ vibration is a transient increase in absorbance in SI followed by a decrease. There is a sustained increase in absorbance in SIl to vibration. The authors conclude that there may be distributed processing of frequencies of skin stimulation in somatosensory cortex. Other techniques like fMRI will certainly be employed in the future, and will become more valuable as their spatial and temporal resolution improve. One problem inherent in all of the imaging studies is that there is not a firm correlation between neuronal activity and the signal being measured. These relationships must be known to evaluate the results of any study using these techniques.

The existence of these responding cortical elements does not directly show their relevance in the process of vibration detection/discrimination. Libet et al. (1964) showed that somatosensory cortex activity occurs in the absence of any perceived sensation. This typically happens when the skin stimulus is applied to the skin at a subthreshold perceptual level, or when elements of the somatosensory cortex are 
stimulated directly by ascending pathways that do not result in a percept. This simply demonstrates that cells exist that are able to entrain to vibration applied to the skin. Several intriguing experiments were designed to answer this question via the techniques of lesioning and intracortical microstimulation. Lamotte and Mountcastle (1979) performed a unilateral removal of the parietal lobe to study its role in vibration detection and discrimination. They found that vibration detection threshold was elevated contralateral to the lesion, and that vibration discrimination was lost. Given greater postoperative time, the amplitude threshold for vibration decreased, but the ability to discriminate was lost throughout the testing period. They concluded that neurons in the parietal lobe were necessary for discriminations within the realm of flutter (defined as frequencies from approximately 20 to $80 \mathrm{~Hz}$ ). Romo et al. (1998) used current pulses injected into SI and tested to see if the monkeys were able to perform pitch discriminations between paired cortical electrical and skin mechanical stimuli. The conclusion they reached was that monkeys were able to use mechanical skin stimulation and cortical electrical stimulation interchangeablyfor the pitch discrimination task. Interestingly, the electrical stimuli need not be periodic to be adequate for discrimination purposes, but their mean frequency must be similar to the simulated mechanical simulation frequency. Romo et al. (1999) also looked for the neurons that would be responsible for the 'hold and compare' feature of the vibration discrimination task (predicted by Mountcastle et al. 1990), and found neurons in the prefrontal cortex that could be responsible for holding the 'memory trace' that occurs in the $1 \mathrm{sec}$ between presented stimuli. 
Another important consideration when examining cortical neuronal responses is the contextual relevance of the stimuli that an animal is receiving. It is possible that neuronal responses could be significantly different in sets of cortical neurons given the attention of the animal to the stimuli being presented. Hyvärinen et al (1980) looked at responses of macaque SI neurons to $40 \mathrm{~Hz}$ vibration of the hand. Animals were either performing a vibration detection task while a light was on, or sitting idly while vibration was applied. This group found that only $16 \%$ of neurons studied had their discharge patterns in response to vibration changed by relevance in the detection task. Nelson et al. (1991) recorded from SI cells that had deep or cutaneous RFs while monkeys did or did not perform a wrist flexion task in response to vibration $(57$ or $127 \mathrm{~Hz})$. They found different effects on activity levels during flexion dependent on RF qualities and cortical areas, stating a combination of variables determine whether attention and context affect SI neuronal responsiveness.

In summary, studies have examined the responses of single neurons to vibrotactile stimuli at most levels of the somatosensory system. These studies have confirmed that these neurons are able to encode vibratory pitch in either the rate or periodicity of their discharges. The point of the studies presented here is to show that neurons exist in the spinal dorsal horn that respond in the same way as neurons in other somatosensory nuclei, and that these neurons may be just as effective at transferring information about vibrotactile stimuli to higher CNS areas as other pathways (e.g. the dorsal columns system, the trigeminal system). 


\section{Methods}

\section{A. Project 1: Responses of dorsal horn cells (DHCs) to transdermal electrical stimuli}

\section{Surgical Procedures}

Cats of either sex $(2.5-5.5 \mathrm{Kg})$ were used. Animals were anesthetized with $15 \mathrm{mg} / \mathrm{kg}$ Telazol. Glycopyrrolate $(.01 \mathrm{mg} / \mathrm{Kg} \mathrm{IM})$ or atropine $(.02 \mathrm{mg} / \mathrm{Kg} \mathrm{IM})$ was administered to minimize cardiodepressant effects of the anesthetic and decrease salivary secretions. The fur of both hindlimbs was clipped to a uniform length of approximately $1 \mathrm{~mm}$. The external jugular vein and carotid artery were cannulated for administration of intravenous drugs and blood pressure monitoring, respectively. Once the venous cannula was in place, alpha chloralose administration was begun. A total dose of $70 \mathrm{mg} / \mathrm{kg}$ was administered in two $1 / 5$ doses twenty minutes apart, followed by $1 / 10$ dose twenty minutes later. $1 / 10$ dose supplements were given every three hours

for the duration of the experiment. A rectal thermister probe was used to measure core body temperature, and temperature was maintained at $38^{\circ} \mathrm{C}$ by a feedback controlled heating pad and heat lamps. Expired $\mathrm{CO}_{2}$ was held at $3.5-4.0 \%$ by controlling respiratory rate.

The deeply anesthetized animal was mounted on a rigid frame, and placed in traction. A lumbar laminectomy was performed to expose the spinal cord at the bottom 
of a mineral oil pool, and all cut bone surfaces were sealed with bonewax. The dura was opened and reflected, and segments $L_{4}-S_{2}$ were identified by tracing dorsal roots. Markers were placed in the musculature adjacent to the spinal canal at Rostrocaudal $(\mathrm{RC})$ levels corresponding to segmental boundaries. This allowed for easy segmental identification during recording.

After the recording was completed, the deeply anesthetized animals were perfused transcardially with $2 \mathrm{~L} 39^{\circ} \mathrm{C}$ saline followed by $2 \mathrm{~L} 10 \%$ formalin at room temperature. Relevant blocks of spinal cord were stored at least overnight in a $5 \%$ sucrose solution. Blocks were mounted on a freezing microtome and sectioned at $50 \mu$. The sections were mounted and stained using a Kluver Barrera stain, and then coverslipped.

\section{Transdermal electrical stimulation of DHC RFs}

Trains of pulses at $5,10,50$ and 100 per second $\left(\mathrm{s}^{-1}\right)$ were generated by laboratory automation software. They were routed to a stimulus isolation unit, which delivered the pulses transdermally via a small (.2mm diameter) pin. The stimulus pin was inserted just far enough to pierce the skin. Pulse duration was 0.1 millisecond, and amplitude was adjusted between .1 and $10 \mathrm{v}$ at a strength that was able to produce DHC responses at $5 s^{-1}$. If the cell was unable to respond to $5 s^{-1}, 10 s^{-1}$ and $50 s^{-1}$ were attempted. If there were no responses that followed these stimuli within the voltage range, the cell was judged unentrainable. Once a stimulus voltage was established, 10 applications of 10 s of pulses at $5,10,50$, and $100 \mathrm{~s}^{-1}$, for a total stimulation time of 
$500 \mathrm{~s}$. There was an interstimulus period of $10 \mathrm{~s}$, so the total time for each characterization was 16.6 minutes. The order of the stimuli was not randomized (i.e. all of the $5 \mathrm{~s}^{-1}$ stimuli were presented, then $10 \mathrm{~s}^{-1}$. etc.). The stimulation paradigm can be found in fig. 1 .

10 Seconds Electrical Pulses Repeat 10 Times

$5 \mathrm{~Hz}$
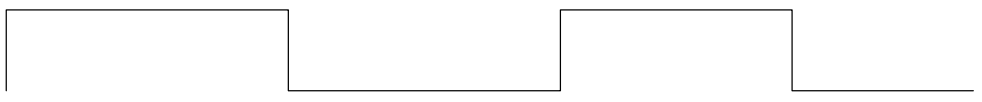

10 Second Intertrial Interval
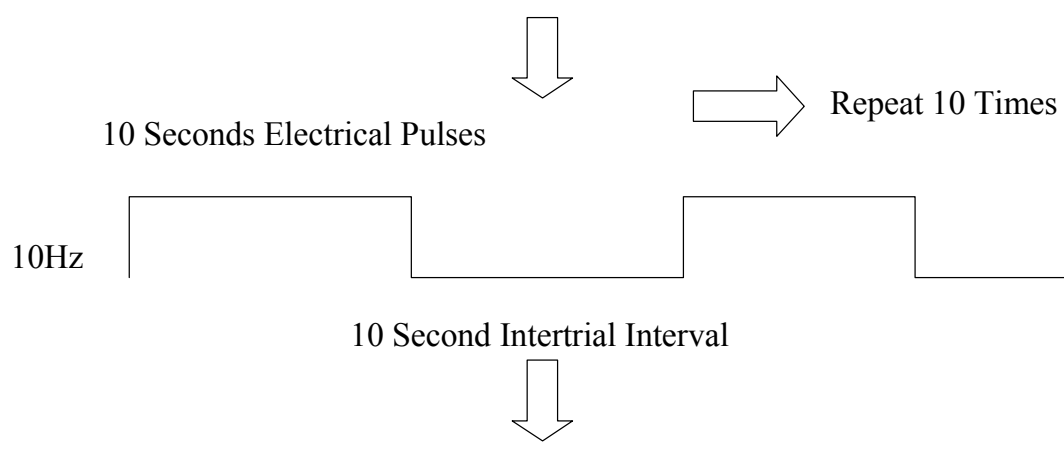

Repeat for $50,100 \mathrm{~Hz}$

Fig. 1. Stimulation paradigm for delivery of electrical stimuli.

\section{Recording of responses}

Responses of DHCs were recorded extracellularly. Stainless steel electrodes were advanced dorsoventrally into the spinal cord with a stepping microdrive. At each rostrocaudal level which was sampled, the first track was just lateral to the dorsal root entry zone (DREZ) and each subsequent track was placed $200 \mu$ medial to the previous 
one (where the overlying vasculature permitted). The procedure was repeated for both sides of the cord, and RC levels were spaced .25 segments apart for ease of electrode track reconstruction. Segmental boundaries were avoided when possible to minimize the loss of tracks when blocking the cord for histological sectioning. DHC responses were recorded in segments L4-S1, the cat lumbosacral enlargement (which contains the hindlimb representation). DHCs with cutaneous RFs were found by manually tapping or stroking the skin while advancing the electrode into the cord. When a cell was isolated as a single unit, its excitatory low-threshold mechanoreceptive RF was drawn on one of three standardized views of the cat hindlimb. The transdermal needle for the electrical stimulus was placed at the center of the identified cell's RF. No variation of stimulus location was attempted in any of these studies.

Signals recorded from the DHCs during electrical stimulation were amplified and routed to a discriminator. The discriminator produced TTL pulses when the level of the microelectrode amplifier output rose above a level specified by the experimenter. The output of the discriminator was used to trigger an oscilloscope showing the microelectrode amplifier output, so that the precise part of the waveform the discriminator was triggering on could be verified. This was done to ensure that only spikes from a single cell were above the discriminator threshold. The output of the discriminator was also routed into a pulse interval timer, which recorded intervals on two channels at a resolution of 10 microseconds (IDAS, Interval Data Acquisition System). The response signal and phase markers were recorded on digital audio tape (DAT) in the last few experiments, to perform offline analyses if necessary, and to serve as an archive of each cell's responses. 
In this series of experiments, only cells which had complete characterizations were used for analysis. A cell was judged to be completely characterized if, after the full characterization, it responded to manual probing of its $\mathrm{RF}$ in a fashion similar to its precharacterization state. This was done to eliminate cells whose responses deteriorated over time.

\section{Analysis of data obtained from electrical stimulation of DHCs}

Analysis methods in these procedures focused on information contained within DHC spike discharge that is available to higher levels of the CNS during periodic stimulation of the skin. The two qualities of the spike train examined in these studies were rate coding (firing rate of cells), and periodicity of discharge. Both have been suggested as possible coding strategies in other parts of the somatosensory system. Another possibility is a place coding system, such as that found in the auditory system. This type of analysis was not possible in these studies, since a sufficient number of stimulus amplitude-frequency pairs was not collected.

\section{a. Histogram generation}

The intervals recorded were used to produce various measures of the degree to which DHCs can follow different frequencies of stimulation. The IDAS used a universal histogram algorithm to generate histograms of several different types (Brown et al., 1985). The three types of histograms chosen for analysis were autocorrelograms, 
phase histograms and post stimulus time histograms (PSTHs). Autocorrelograms can be analyzed to show periodicity in a cell's discharges, PSTHs can show the time course of responses during the entire stimulation period, and phase histograms can be used to look at timing of responses relative to each stimulus presentation.

In this series of experiments, the electrical stimulus produced a large artifact that was much greater than the amplitude of $\mathrm{DHC}$ action potentials; the discriminator produced a pulse for every stimulus artifact. The counts for the artifact had to be removed to avoid contaminating the sample of real action potential events. This was accomplished by examining the phase histogram, and editing it such that all response events falling within the first millisecond or two following the phase marker were removed. This may remove some activity from the overall histogram, as the stimulus artifact in principle could be concurrent with neural events. However, latencies of the stimulus artifact were well below those of the cell responses (due to synaptic delay and conduction distance), so very little of the 'driven' response was removed. The stimulus artifact would have skewed the data analysis significantly, considering that it is perfectly locked to the phase marker in a 1:1 fashion.

\section{b. Analysis of interval files}

Interval files (files containing channel number and time since last incoming event) were used for other data analysis methods as well. Analysis of the responses fell into two broad classes: periodicity analyses and firing rate analyses. 
Both analyses can give insight into how the response is related to the stimulus frequency, and the two types of information are not necessarily related (i.e. a cell that responds with a high firing rate may have very little periodicity in its discharge).

Periodicity in neuronal responses was examined in two ways: phase vector analysis and power spectra. Phase vectors provide a means of looking at how well the response is entrained by the stimulus, and power spectra show which frequencies are prevalent in spike trains. The CNS has no knowledge about the stimulus other than that provided by sensorineural discharge patterns.

\section{c. Vector strength analyses}

Phase vectors are calculated by converting the timing of each response (action potential) into a unit vector with a phase angle determined by the time of response during the stimulus period; these are summed.

$$
\mathrm{r}=1 / \mathrm{n}\left[\left(\sum_{\mathrm{i}=1}^{\mathrm{n}} \sin 2 \pi \mathrm{ft}_{\mathrm{i}}\right)^{2}+\left(\sum_{\mathrm{i}=1}^{\mathrm{n}} \cos 2 \pi \mathrm{ft}_{\mathrm{i}}\right)^{2}\right]^{1 / 2}
$$

Where $r=$ average vector strength for all action potentials over all stimulus cycles $\mathrm{n}=$ number of action potentials $f=$ frequency of stimulation (inverse of period) $t_{l}=$ time of action potential relative to previous zero phase marker

The resultant vector has a magnitude $r$ and a phase angle. The phase angle is given by: 


$$
\begin{aligned}
& \mathrm{x}_{\mathrm{i}}=\cos \left(2 \pi \mathrm{ft}_{\mathrm{i}}\right) \\
& \mathrm{y}_{\mathrm{i}}=\sin \left(2 \pi \mathrm{ft}_{\mathrm{i}}\right) \\
& \Theta=\arctan \left(\sum \mathrm{y}_{\mathrm{i}} / \sum \mathrm{x}_{\mathrm{i}}\right)
\end{aligned}
$$

where $\mathrm{x}=\mathrm{x}$ component of each action potential

$$
\begin{aligned}
& y=y \text { component of each action potential } \\
& \Theta=\text { Phase angle for resultant vector }
\end{aligned}
$$

A vector strength of one indicates that all responses fall at the same time relative to the phase marker. A vector strength of zero indicates that the distribution of times for all responses is even throughout the stimulus cycle period. An example of all of the unit vectors and the resultant vector for a cell's response at $5 \mathrm{~s}^{-1}$ stimulation are shown in fig. 2.

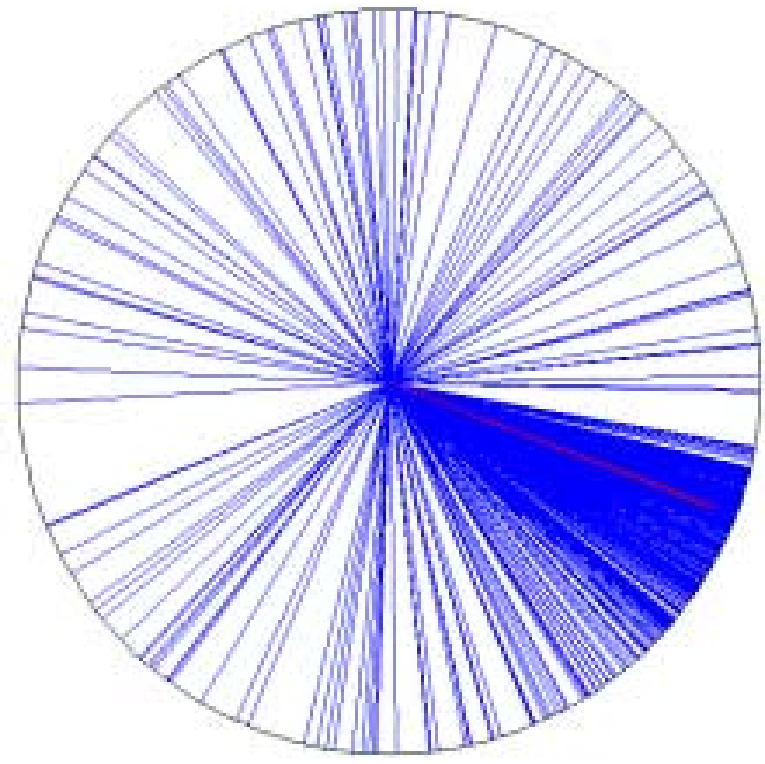


Fig. 2. Action potential unit vectors (blue) and resultant phase vector (red) for one DHC response during $5 p p s$ stimulation.

Vector strengths were calculated for the responses of every cell at each stimulus frequency $(5,10,50$ and 100pps). Plots of vector strengths for all cells at all frequencies were generated. Mean vector strengths and standard deviations were calculated for responses to each frequency of stimulation. A statistical test commonly employed to look at the coherence of vectors is Rao's spacing test (Batschelet, 1981). This test assigns a level of significance on the dispersion of the vectors in a given sample. A significant result means that the sample is not evenly distributed, or that some directedness exists. This test was performed on all of cells responses at each frequency.

\section{d. Power Spectra}

The second measure of periodicity performed was construction of power spectra of $\mathrm{DHC}$ responses. The first step was to construct an autocorrelogram for the responses of each cell to each frequency. Fast Fourier transform software was used to calculate the power spectrum periodogram of the autocorrelogram. This shows the spectral density of all frequencies present in the autocorrelogram. The power of each

bin was tabulated, and a highest power-frequency (principal frequency component) was found for each power spectrum. The power of the principal frequency component was 
divided by the power of all the frequency bins to yield a percent power for the principal frequency in the response. This measure gives a value for the principal frequency component of each responding cell, and this value can be compared with the frequency of stimulation to determine whether or not there is a relationship.

\section{e. Measures of firing rates in cell discharges}

Firing rates of DHCs were determined by summing the total number of spikes recorded and dividing that number by the total stimulation time, which was 100 s in this series of experiments. Thus, the term 'firing rate' refers to the average firing rate of one cell during its entire stimulus period. 'Mean firing rate' refers to the average of all cells' firing rates.

Firing rates of cells in response to electrical stimuli were characterized in several ways. Line plots of firing rates for each cell vs. frequency and mean firing rate vs. frequency were generated. Distributions of cell firing rates were also plotted as histograms for each frequency. Percentages of cells that respond at a rate greater than the frequency of stimulation were also plotted. This analysis was done since DHCs often respond with a burst of action potentials to single pulsatile stimuli applied to their RFs.

An attempt was made to examine the relationship between firing rate and periodicity. Plots of firing rate vs. frequency were used to show the correlation (correlation coefficient) between the two variables. 


\section{f. Adaptation}

It is possible that these cells are able to follow the stimuli well for a limited time during the beginning of a stimulation period. If the cells exhibit adaptation, then the ability of the cell to follow the stimulus will be lost when data for an entire stimulus period is analyzed. To look at the early response of the cells, the phase locking and firing rates were calculated for the first ten cycles of the applied stimulus. This gives the response characteristics during the very early portion of the stimulus. Firing rates were calculated by multiplying the number of spikes during the first ten cycles, and dividing that number by the total time of stimulation, e.g. for $5 \mathrm{pps}$, the number of spikes was divided by 2 seconds, since 10 cycles of a $5 p p s$ stimulation has a period of 2 seconds. Analysis methods for firing rate and phase vectors were identical to those used for the full 10 s characterization.

\section{g. Population responses to electrical stimuli}

In these studies it is important to examine the responses of the population of neurons to each stimulus frequency. The responses of all cells to each frequency of periodic electrical stimulation were combined in order to model the afferent inflow the CNS receives during this type of stimulation. The responses of all cells were combined by creating a combined interval file in which the first phase marker in each individual cell file was taken as time zero, and all subsequent action potentials in all cells' interval files were added to the combined file. This file thus contained all spikes in all cells in response to one frequency of electrical stimulation. It is important to note 
that no correction was made for conduction path (i.e. distance of recording site on the skin to the recording electrode). Autocorrelograms of the combined responses were generated, and FFT software was used to generate power spectra of the autocorrelograms. Mean conduction velocity was calculated by creating a scattergram of latency vs. RF distance from tips of toes. 


\section{B. Project II: Responses of DHCs to sinusoidal mechanical stimuli}

\section{Surgical Procedures}

Surgical procedures used for this series of experiments were very similar to those described for the electrical stimulation study. One exception is that the hindlimbs of each animal were secured to a point further away from the stereotaxic frame. This was done to minimize the angle between the table and the hindlimb, and allow access to more of the dorsal surface of the leg with the mechanical stimulator. Even with this compensation, many areas of the leg were inaccessible to the stimulus unit.

\section{Sinusoidal cutaneous stimulation}

A programmable waveform generator (Videospectra model 1010 synthesized function generator) generated sine waves of varying amplitude and frequency. The sine waves generated were at frequencies of $5,10,50$ and $100 \mathrm{~Hz}$, at amplitudes that corresponded to $70,150,300$ and $500 \mu \mathrm{m}$ of displacement.

The output of the function generator was routed to a ramp generator that used an analog multiplier to modulate the sine wave with an internally generated trapezoid. Thus, the stimulus onset was gradual, not sharp. The duration of the trapezoid ramp rise time was fixed at $110 \mathrm{msec}$ (total trapezoid duration $=5 \mathrm{~s}$ ). TTL pulses produced 
at the positive zero crossing of the sine wave input served as phase markers for subsequent histogram generation.

The stimulus burst was then routed to a feedback-controlled mechanical stimulation unit (BME Systems Vibrotactile Stimulator). This unit was set in controlled displacement mode, meaning that the displacement waveform was proportional to the control waveform regardless of variations in load. The probe tip was cylindrical, and had a diameter of $5 \mathrm{~mm}$. No attempt was made to examine the effect of probe tip size on responses of DHCs. The stimulator had a monitor output that displayed the movement of the probe tip, and the sine waves were produced faithfully even in the face of considerable resistance (observed by pressing on the tip during a test stimulus and observing the monitor output). The stimulator was unable to produce a $200 \mathrm{~Hz} 500 \mu$ stimulus for the required stimulation period, so applications of $200 \mathrm{~Hz}$ stimuli ranged from $70-300 \mu m$ in amplitude. The stimulator resting position was controlled by an xyz manipulator (Narashige) which was mounted to a heavy base. The mass of the entire apparatus afforded mechanical stability for stimulus placement throughout a characterization.

The paradigm used for the mechanical characterization procedure is described diagramatically in fig. 3. The stimulus duration for each trial was $5 \mathrm{~s}$, followed by an interstimulus interval of $5 \mathrm{~s}$. A total of ten applications of $5 \mathrm{~s}$ of each stimulus-amplitude combination were applied, for a total characterization time of 33.3 minutes. The order in which the stimulus-amplitude pairs were applied was pseudorandomized for each experiment. This was done to minimize any temporal effects during the course of the 
characterization (due to variable physiology of the cell, physiological variation of the animal, long term adaptation, etc.).

\section{Recording of Responses}

The electrophysiological techniques used for recording from DHCs were identical to those used in the electrical stimulation study. Once a single unit was isolated, the mechanical stimulator was positioned so that the probe tip was contacting the center of the DHC RF. The probe tip was positioned so that its surface was as normal to the skin as possible. Due to the curvature of the leg, complete contact between the probe tip and the skin was impossible in some areas. The tip was always positioned to maximize contact with the skin. Test stimuli of $5,10,50$ and $100 \mathrm{~Hz}$ were applied at an amplitude of $500 \mu$ to determine what pre-indentation was necessary for the cell to respond.

Typically, an indentation was necessary to get the cells to respond to the stimulus. The indentations used in this study ranged from 0 to $1.5 \mathrm{~mm}$. The neutral position of the stimulator tip was pressing down on the clipped mat of fur, just touching the underlying skin. The term 'indentation' refers to the amount the tip was advanced after contacting the clipped mat of fur, not the skin itself. Another factor that determined the amount of indentation necessary was the mechanical coupling at the stimulation site. On areas of the leg overlying bone, the force delivered by the stimulator was much greater than at sites overlying muscle or fat. 
Ramp duration:110 msec

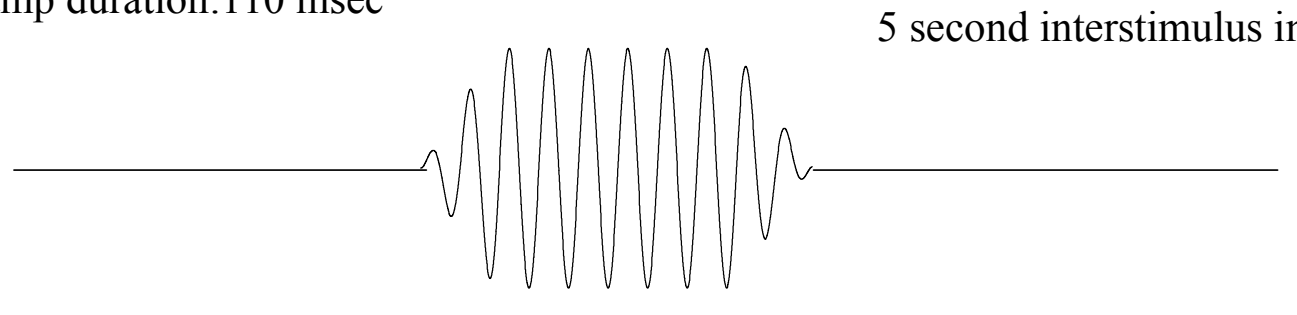

5 seconds sinusoidal stimulation

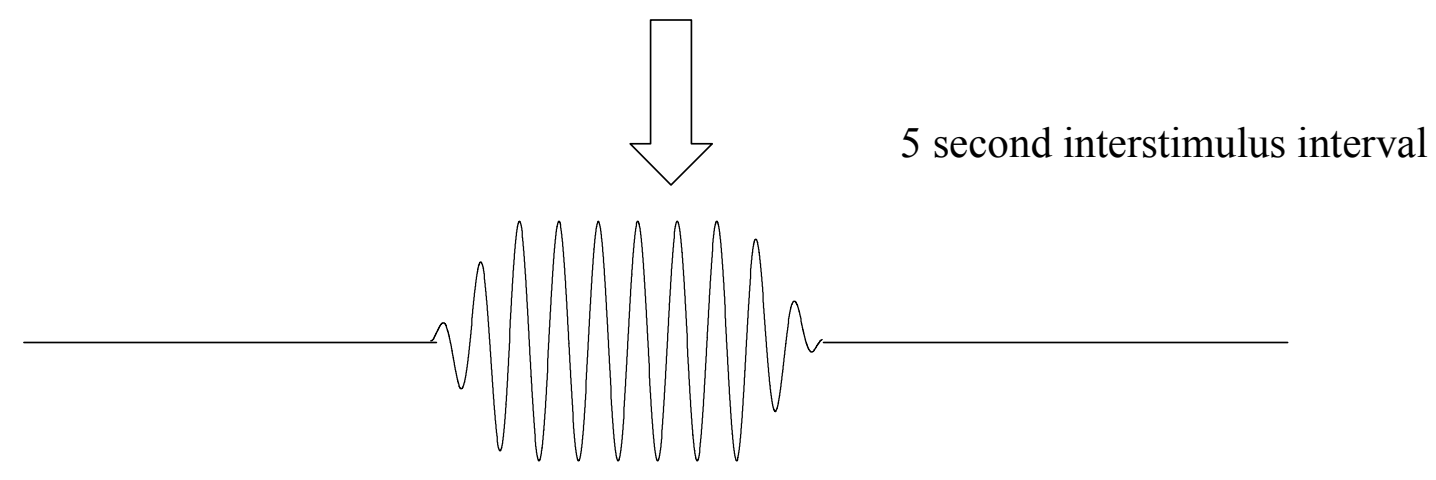

5 seconds sinusoidal stimulation, amplitude and frequency determined by next pairing on pseudorandom

list

Fig. 3. Mechanical stimulation paradigm.

Only cells with complete characterizations were used in this study. Many isolated units had RFs which were inaccessible to the stimulator, so the RFs of the DHCs characterized tended to be on the posterior and lateral surfaces of the hindlimb.

\section{Data analysis methods}

Analysis methods were performed in exactly the same way as in the electrical study, with the exception that stimulus amplitude had to be dealt with as well as stimulus frequency. 


\section{Results}

\section{A. Electrical stimulation of DHC RFs}

Full characterizations of 54 DHCs were obtained in 7 animals. 95\% (19/20) of the reconstructable cells were located in laminae III and IV, where cells are known to have low-threshold mechanoreceptive input (reviewed in Brown, 1981). RF locations of characterized cells are shown on standard leg views in fig. 4.

\section{Histogram analysis}

Phase histograms generated online were used for analysis of firing rate and periodicity calculations. PSTHs for each cell were used to examine the response during the entire stimulus period. Fig. 5 shows the PSTHs of the responses of 3 cells

during a $5 \mathrm{~s}^{-1}$ stimulation. The response remains fairly consistent throughout, suggesting that no adaptation occurs during application of this low stimulus frequency. Figs. 6-8 show the same types of histograms, each for one frequency of stimulation. It is apparent that 


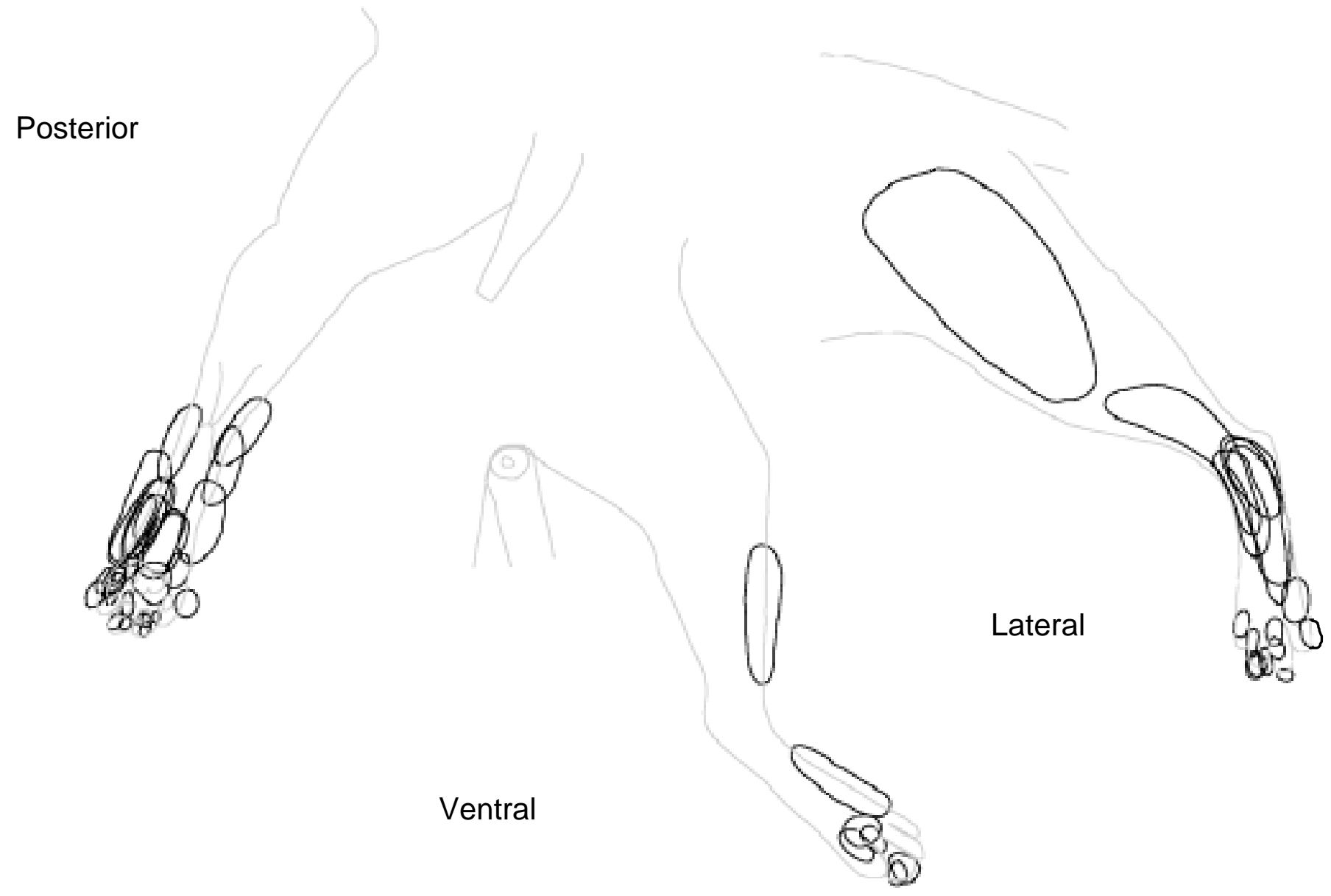

Fig. 4. RFs of DHCs in transdermal electrical stimulation study. 
A

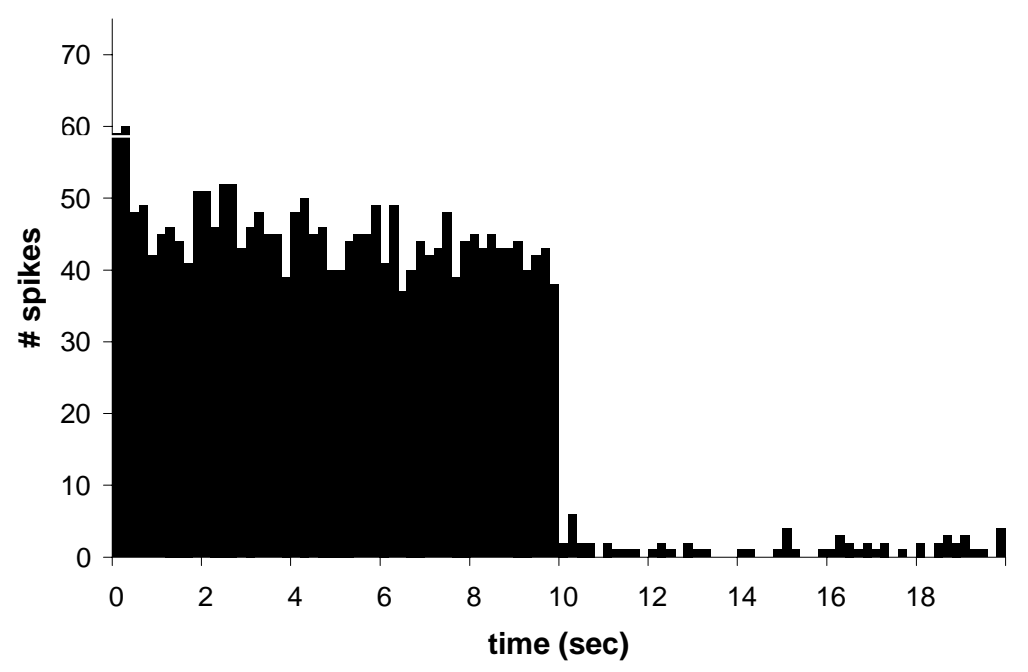

C

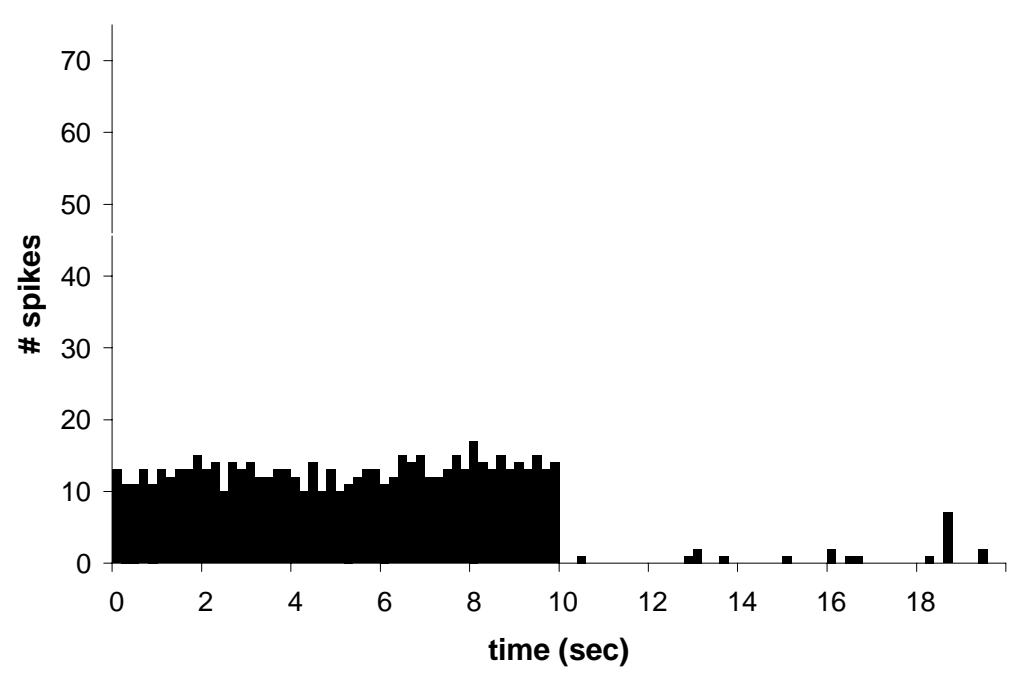

Fig. 5. PSTHs of three DHC responses to $5 \mathrm{~s}^{-1}$ stimulation.

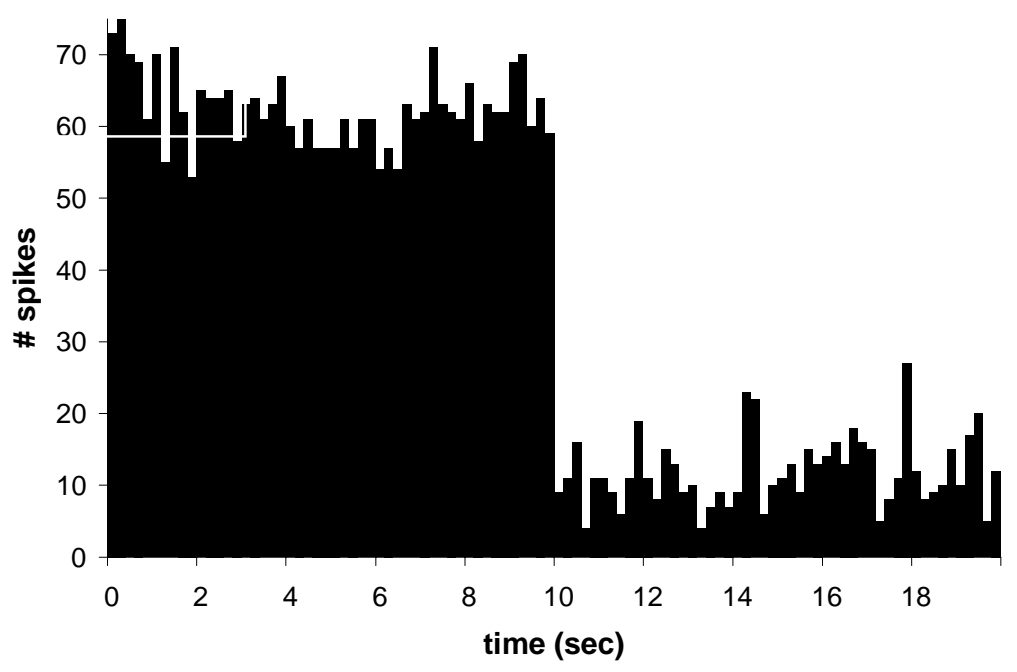


A
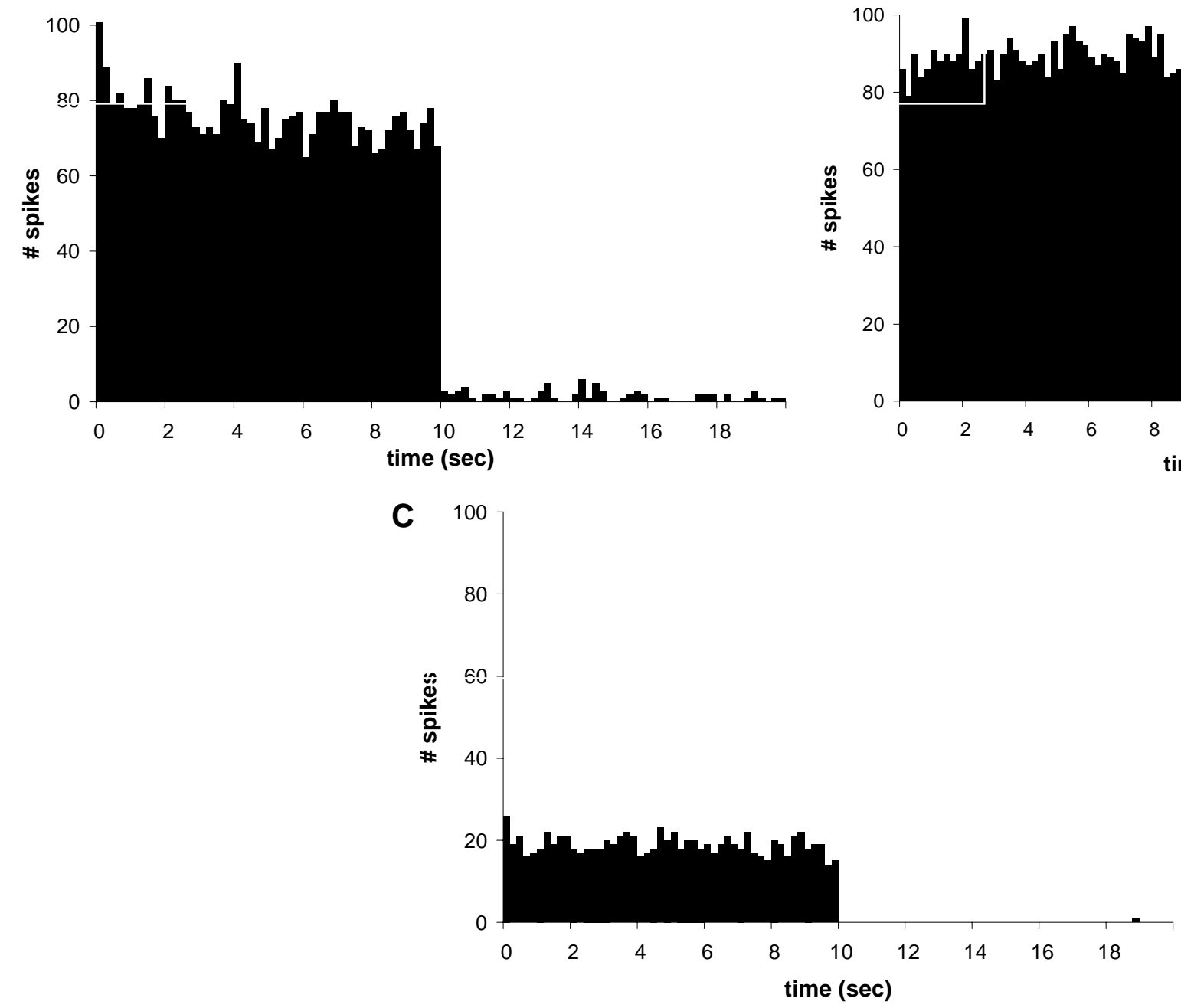

Fig. 6. PSTHs of the same three DHCs responses to $10 \mathrm{~s}^{-1}$ stimulation. 
A

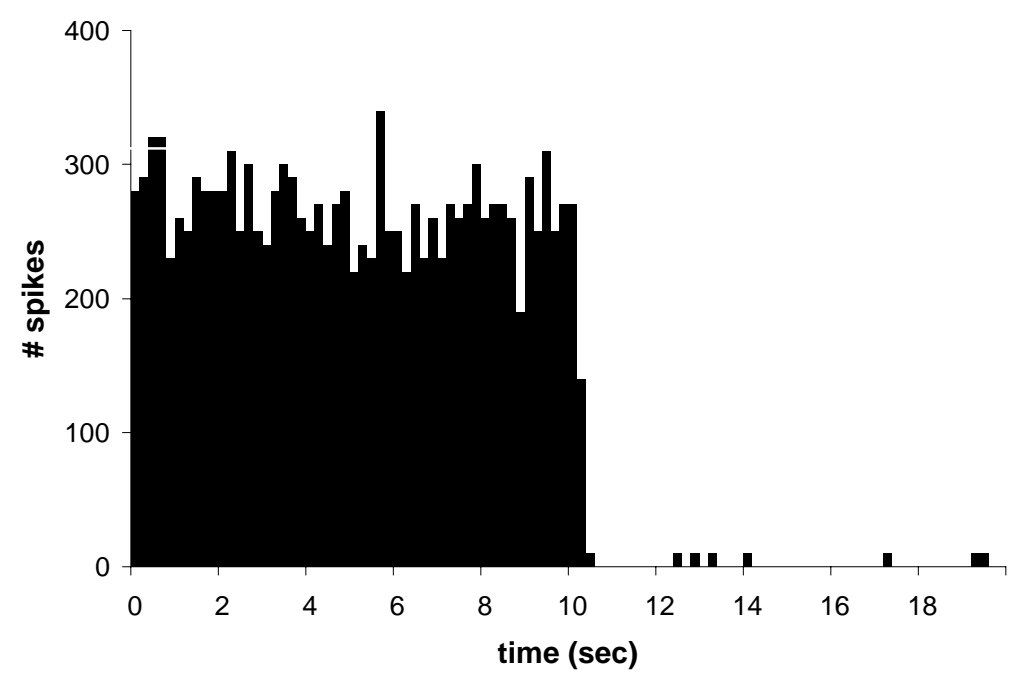

C

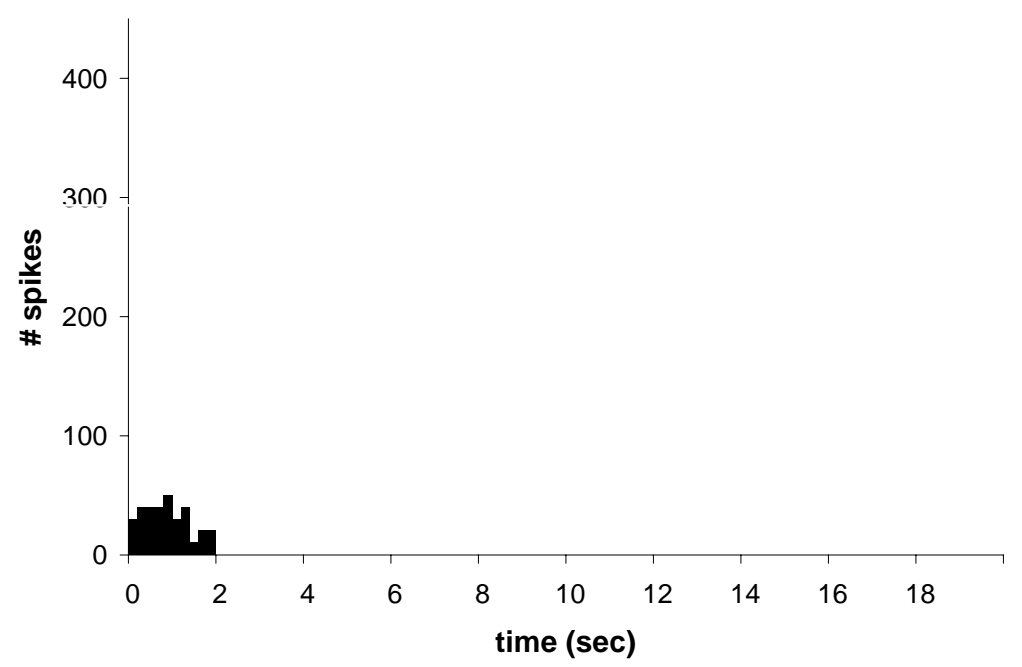

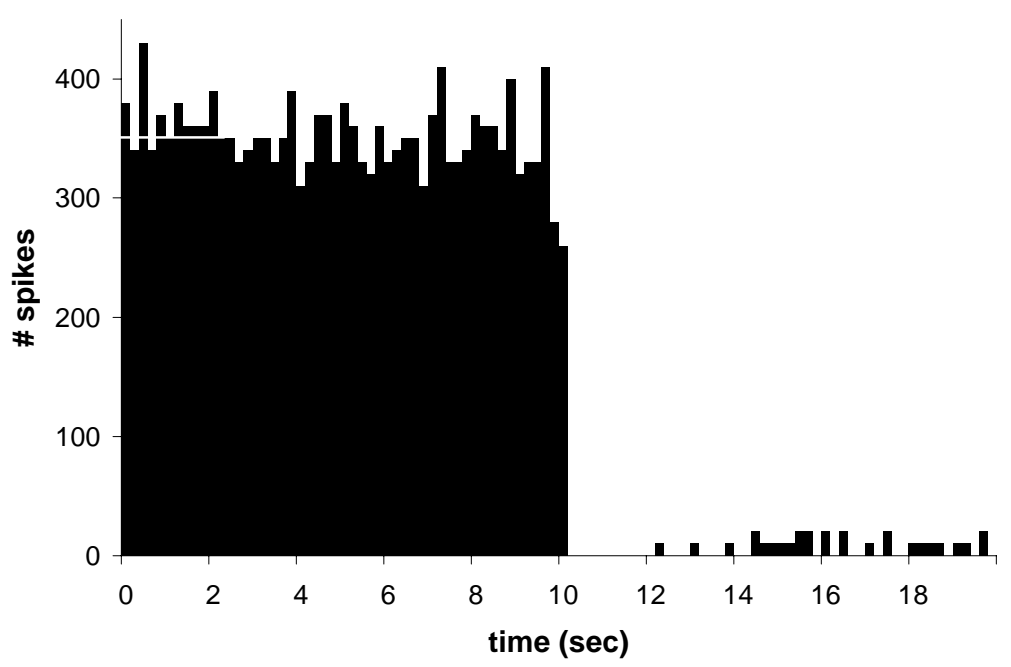

Fig. 7. PSTHs of three DHC responses to $50 \mathrm{~s}^{-1}$ stimulation. 
A

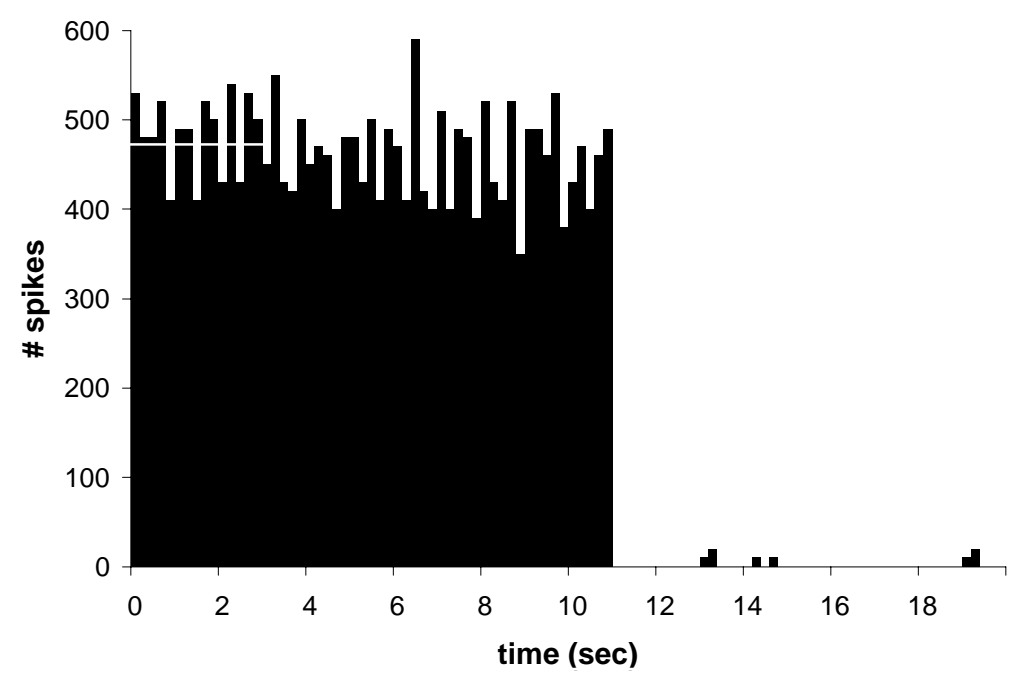

C

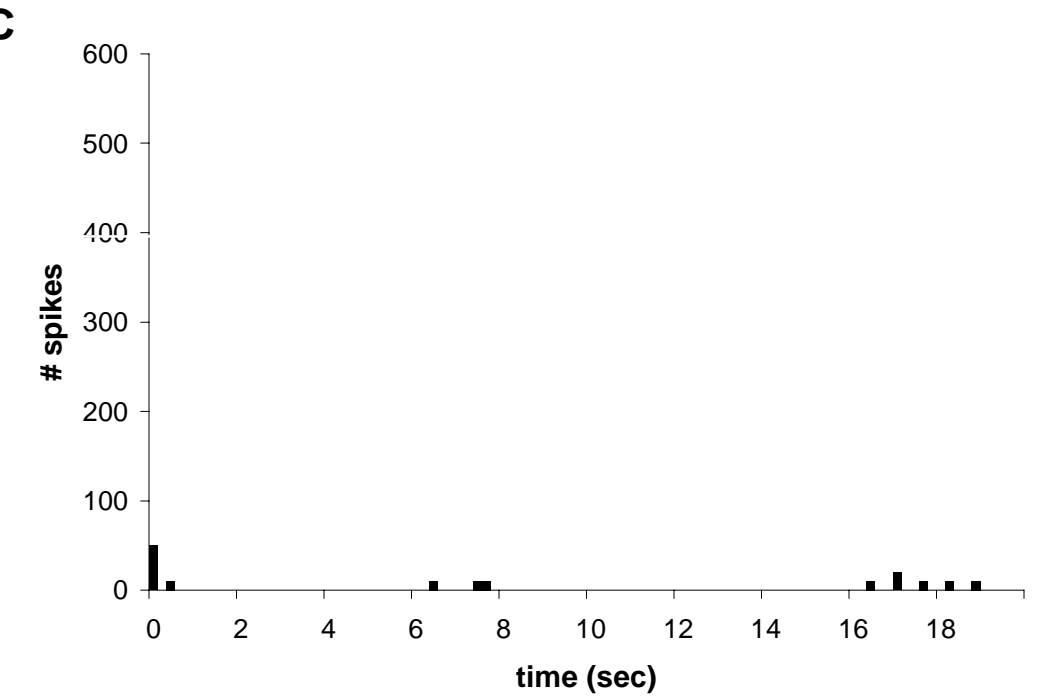

Fig. 8. PSTHs of three DHC responses to $100 \mathrm{~s}^{-1}$ stimulation. 
the degree of attenuation in the response increases as stimulus frequency increases in cell C. However, A-B show another type of cell that was able to respond well throughout the entire stimulation period up to $50 \mathrm{~s}^{-1}$. There was a small number of neurons in this study that were able to follow high frequencies of stimulation very well, and others that were able to follow up to 10 or $50 \mathrm{~s}^{-1}$. There were relatively few cells that did not adapt during $50 \mathrm{~s}^{-1}$ stimulation (18.5\%), and even fewer during $100 \mathrm{~s}^{-1}$ stimulation (7.4\%).

\section{Vector strength analysis}

Fig. 9 shows the phase vectors for all cells at $5,10,50$ and $100 \mathrm{~s}^{-1}$ stimulation. The increasing dispersion in the phase angles of the vectors with increasing stimulus frequency is due to the shortening of the period of the stimulus. The latencies due to conduction velocity differences will be much more apparent in the dispersion of phase vectors at $100 \mathrm{~s}^{-1}$, because the period is only $10 \mathrm{msec}$, whereas at $5 \mathrm{~s}^{-1}$ the period is 200 msec. The length of the individual phase vector shows the phase locking of responses from one cell, and summary histograms of vector lengths across all cells are shown in fig 10. A-D. There is no statistically significant trend, due to the large standard errors.

The results for Rao's spacing test for directedness are shown in figure 12. At the $p<.01$ level, all four frequencies of electrical stimulation produced cell responses whose phase angles were not uniformly distributed. This means that the responses fell into a cohesive time period following the phase marker. 
A

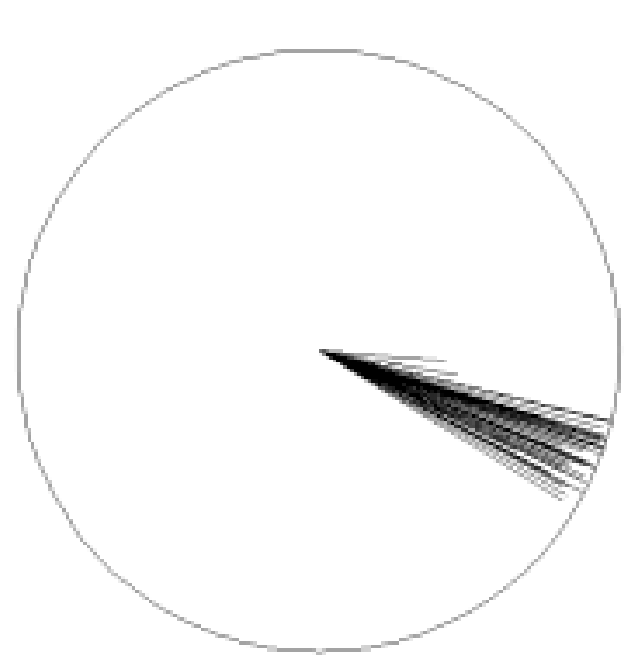

C

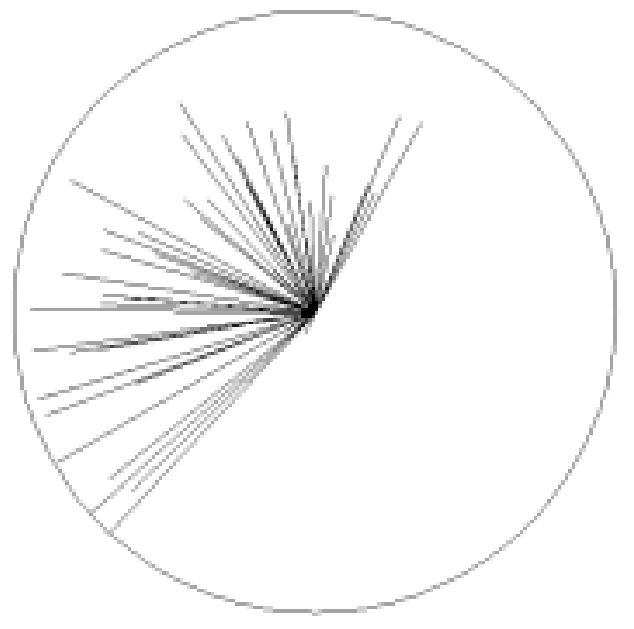

B

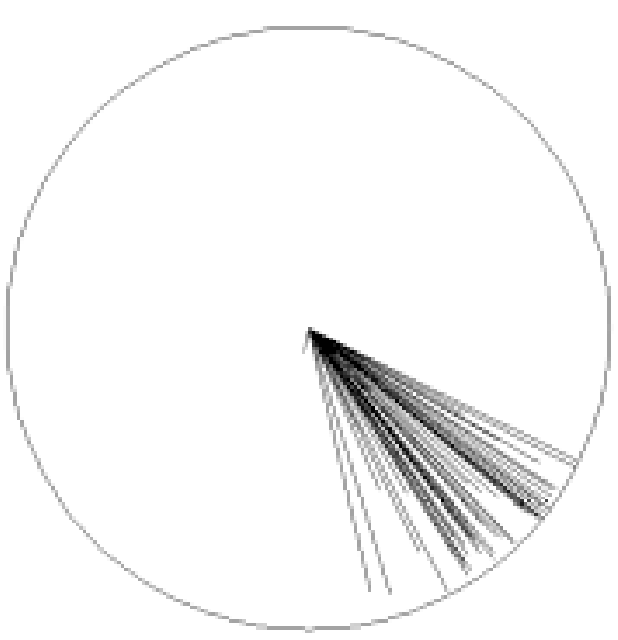

D

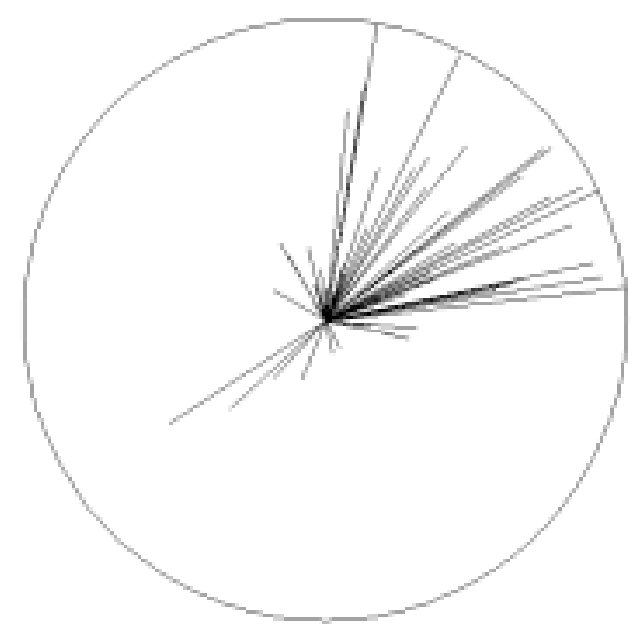

Fig. 9. Phase vector diagrams of $\mathrm{DHC}$ responses to 4 rates of electrical stimulation. A-D: $5,10,50$ and $100 \mathrm{~s}^{-1}$ 

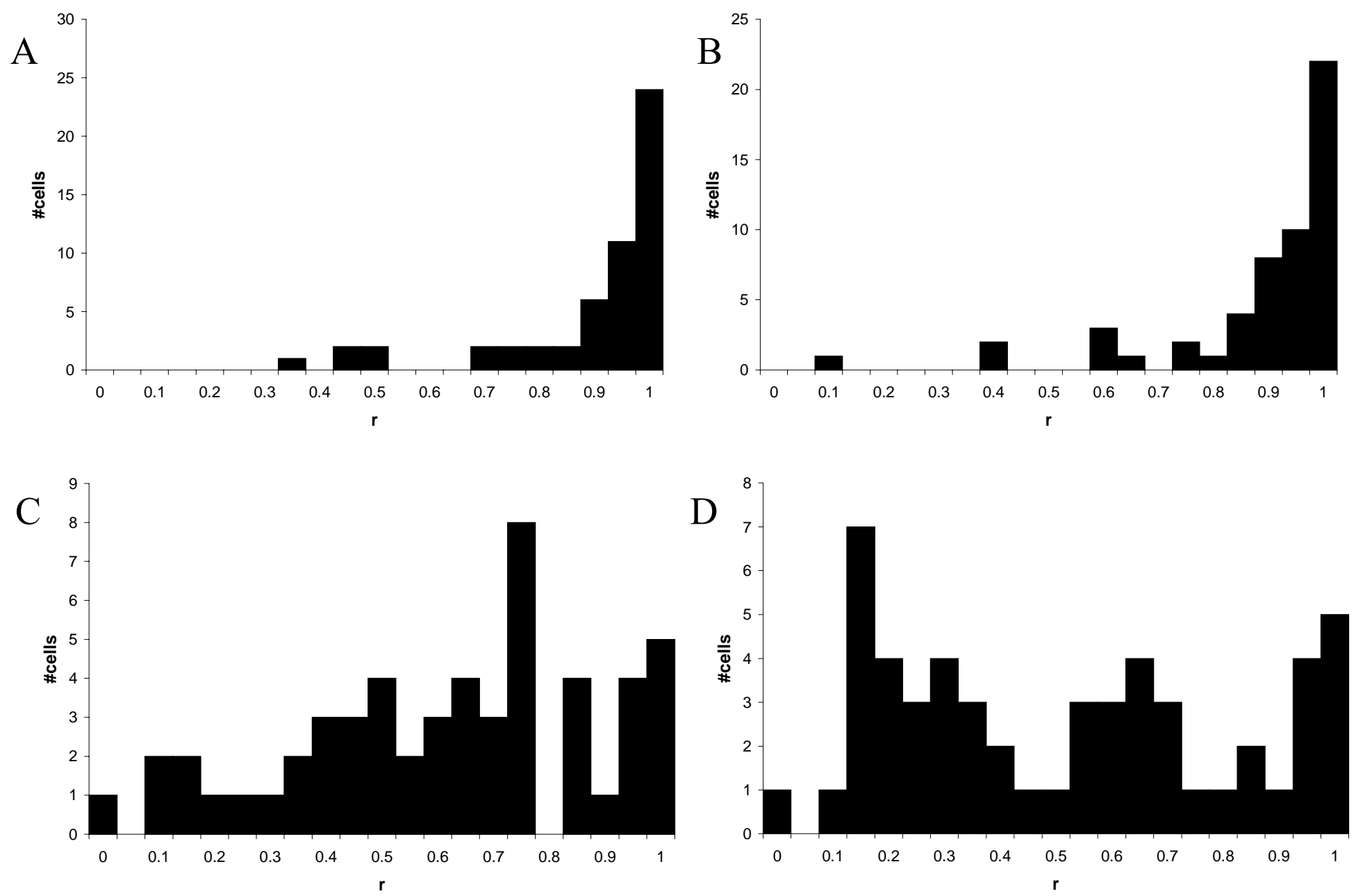

Fig. 10. Summary histograms for vector strengths of $D H C$ responses to (A-D) $5,10,50$ and $100 \mathrm{~s}^{-1}$ stimulation. 


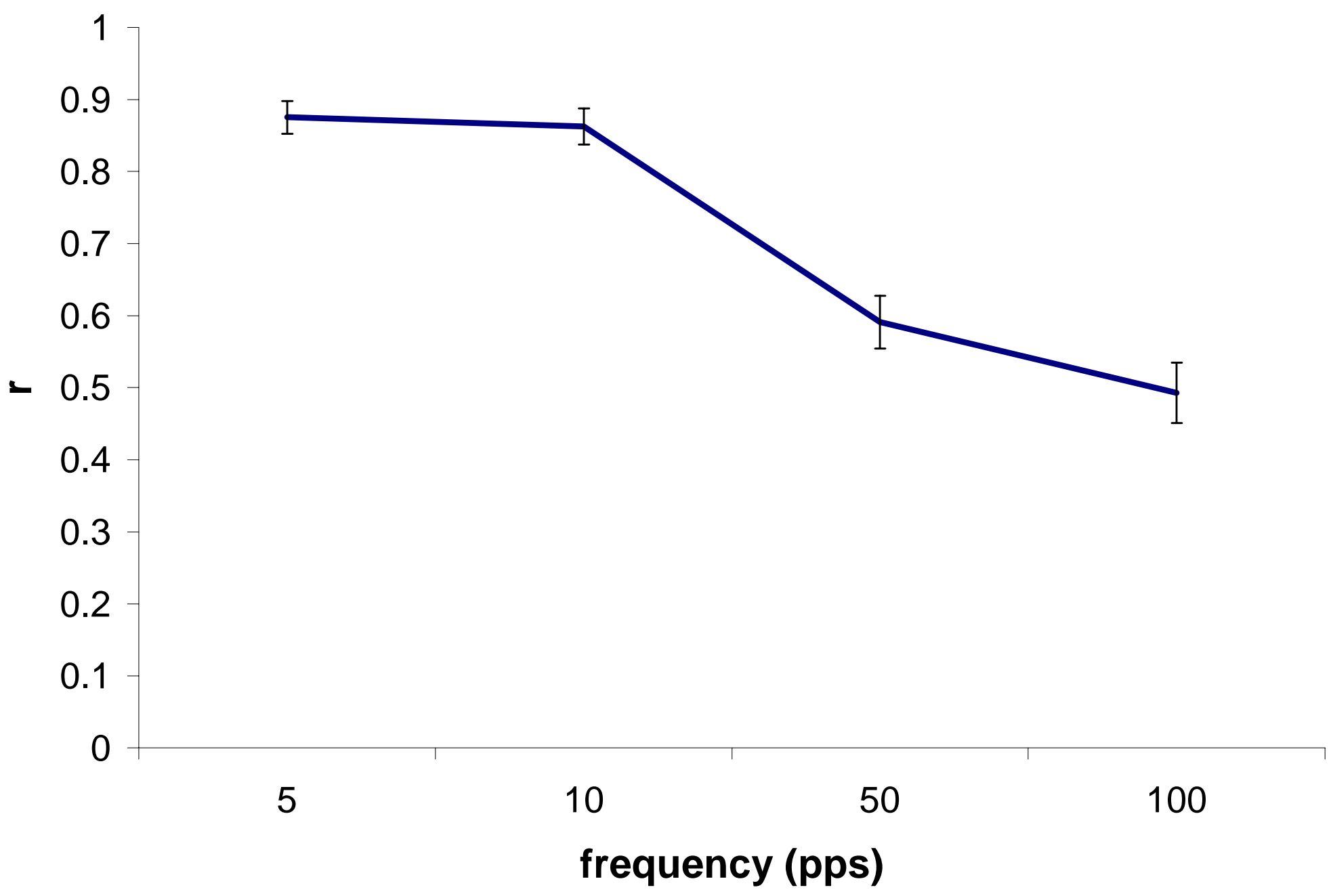

Fig. 11. Mean phase vector strengths for all DHC responses at four frequencies of electrical stimulation. 


\begin{tabular}{|c|c|c|}
\hline Frequency $(\mathrm{Hz})$ & Test Statistic $(U)$ value & Significance Level, $p<$ \\
\hline 5 & 326.95 & 0.01 \\
\hline 10 & 296.24 & 0.01 \\
\hline 50 & 186.87 & 0.01 \\
\hline 100 & 185.78 & 0.01 \\
\hline
\end{tabular}

Fig. 12. Rao's spacing test results for directedness of phase vectors.

The magnitudes of the phase vectors shown in fig. 9 are far more informative than the phase angles associated with each vector. The mean vector strength at each rate of stimulation is shown in fig. 11 . The mean phase vector is maximal at $5 \mathrm{~s}^{-1}$, with a value of .86. The standard error is also lowest at this stimulation rate, showing that most of the cells seem to be both very well phase locked and consistent across cells. There is a drop-off of $r$ and inverse of standard error at $50 \mathrm{~s}^{-1}$ and $100 \mathrm{~s}^{-1}$. During these stimulation rates, the mean vector strength is less than 0.6 and there is a much higher standard error.

\section{Analysis of Power Spectra}

An example set of autocorrelograms for one cell's responses is shown in fig. 13 This cell is responding to transdermal electrical stimulation at 4 frequencies. The responses clearly are falling at intervals commensurate with stimulus frequency (e.g. $200 \mathrm{msec}$ for $5 \mathrm{~s}^{-1}$ stimulation). Phase histograms for this cell are shown in fig. 14 
Corresponding power spectra for these autocorrelograms are shown in fig. 15 The principal frequency components in each graph cluster around the fundamental harmonics and sub-harmonics of the stimulus frequency. This gives an indication that periodicity is being preserved to at least some degree in these cells, and that the periodicity in the spiking patterns is related to the stimulation frequency. The scatter of the peak response frequencies during high $\left(100 \mathrm{~s}^{-1}\right)$ frequency stimulation is higher than that seen in responses during low $\left(5 \mathrm{~s}^{-1}\right)$ frequency stimulation.

Fig. 16 shows the distribution of principal frequency components for the responses of all cells at 4 frequencies of stimulation. At $5 s^{-1}$, the peak response frequencies occur almost exclusively at the frequency of stimulation and its harmonics. As the frequency of stimulation increases, peak responses in the power spectrum show a greater number of non-harmonic frequencies.

Coefficients of variation calculated for the peak frequencies are shown in fig. 17 There doesn't seem to be any real trend that correlates with frequency, as the coefficient rises from $5-10 \mathrm{~s}^{-1}$ stimulation, but subsequently drops from 10 , to 50 , and then $100 \mathrm{~s}^{-1}$. The coefficients of variation do not provide much information. 
A

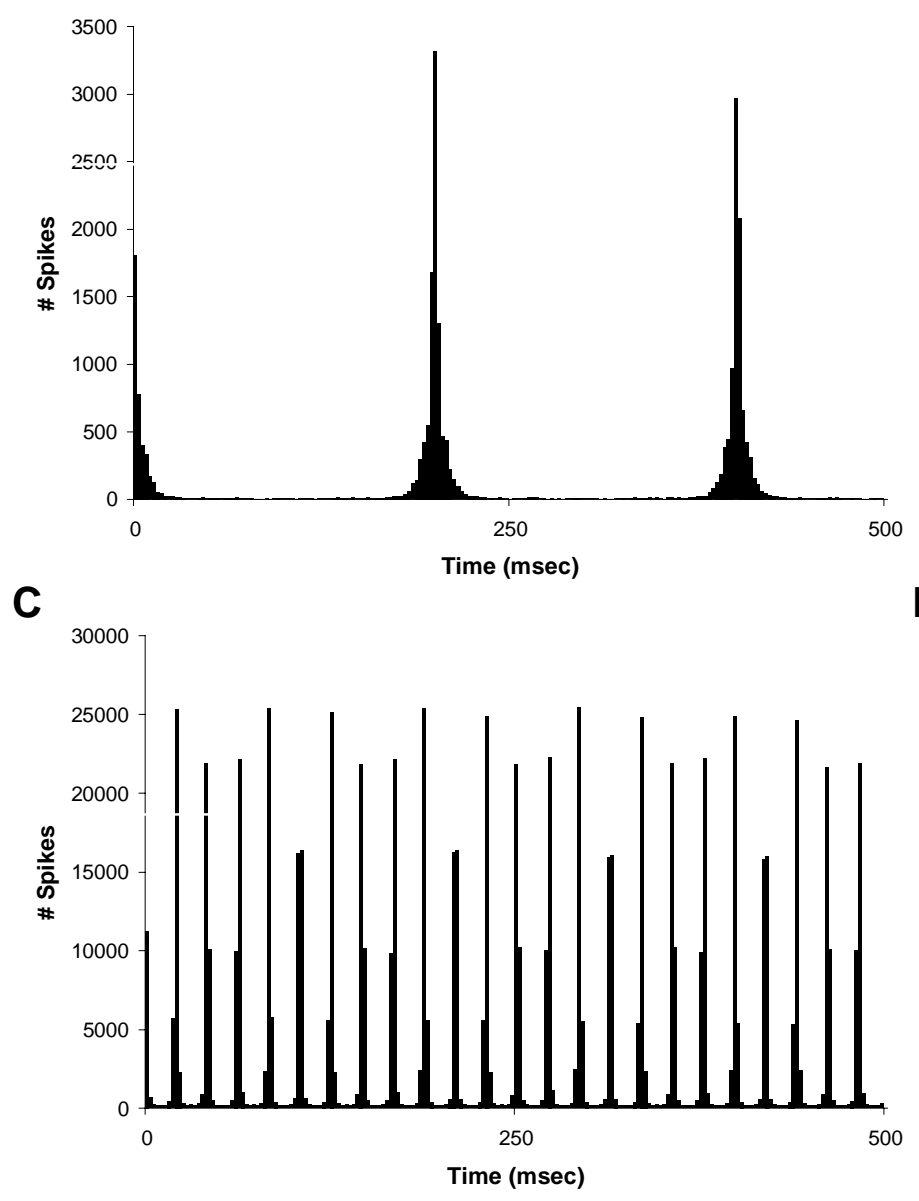

B

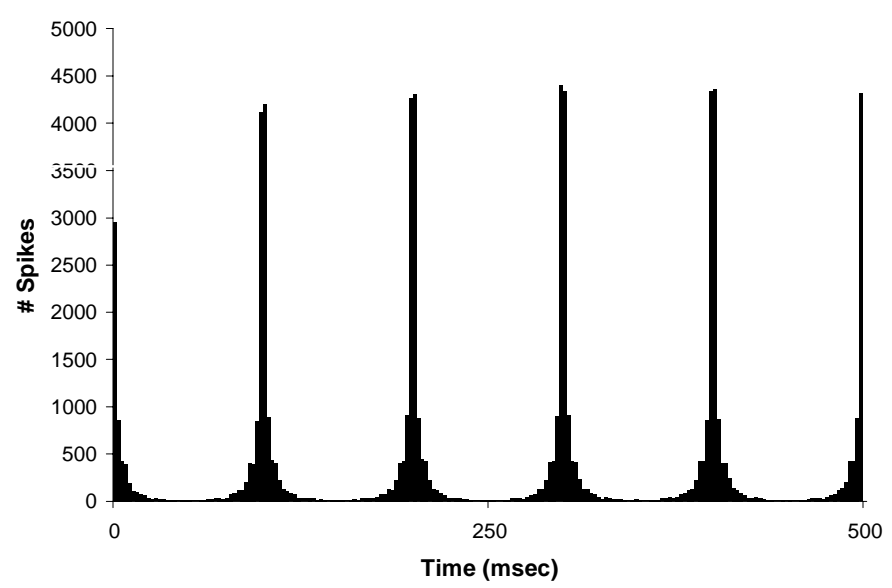

D

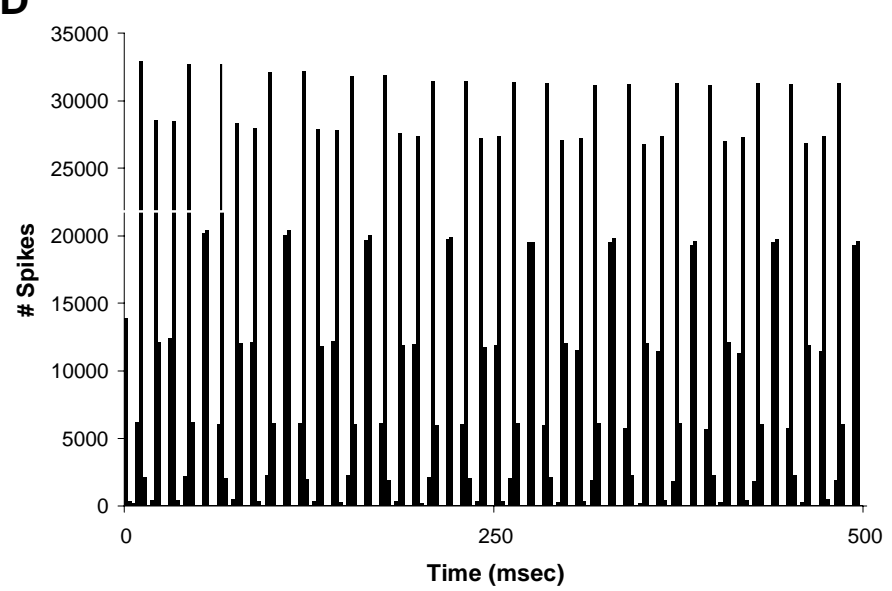

Fig. 13 Autocorrelograms of responses of one $\mathrm{DHC}$ to (A-D) 5, 10, 50 and $100 \mathrm{~s}^{-1}$ stimulation. 
A
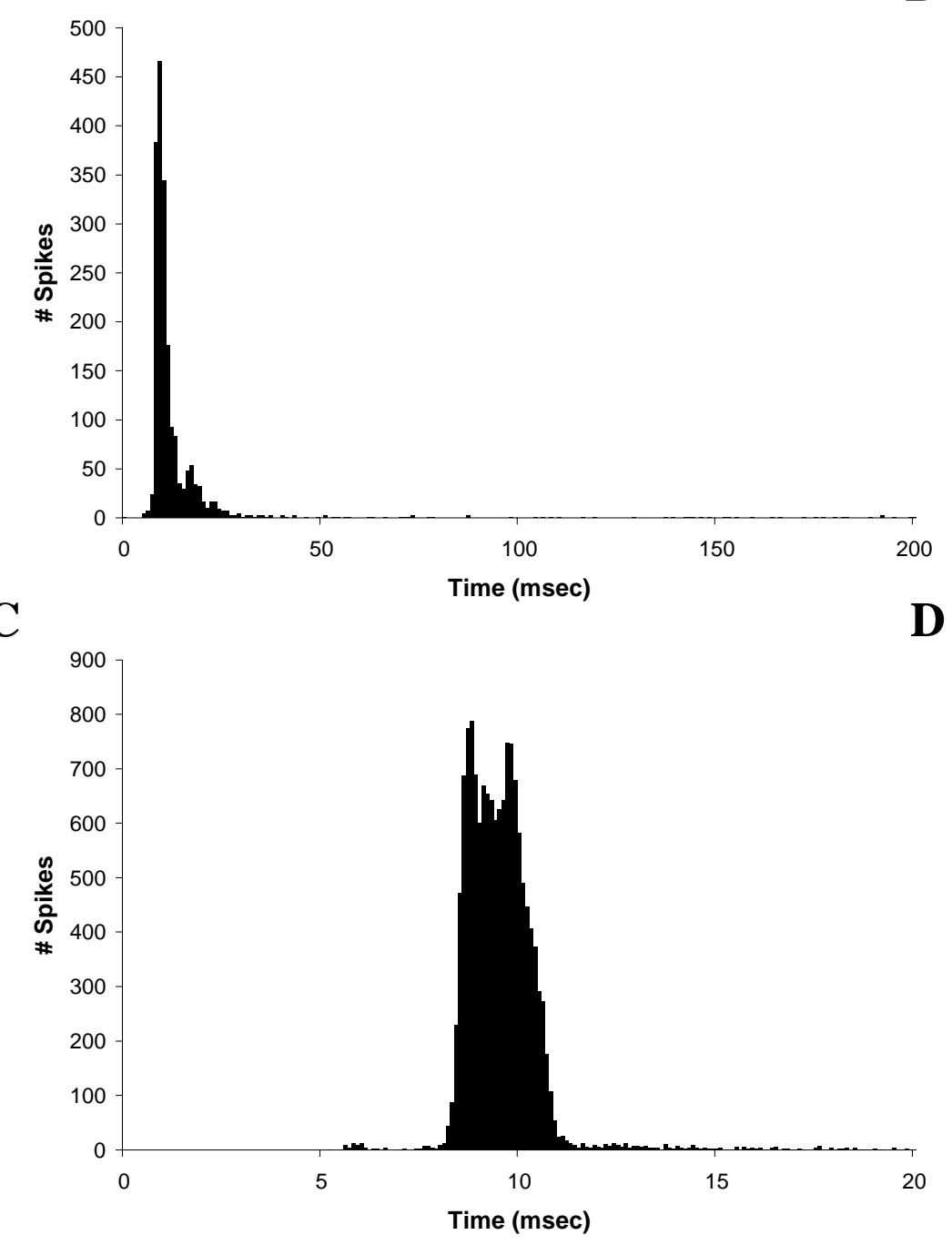

B
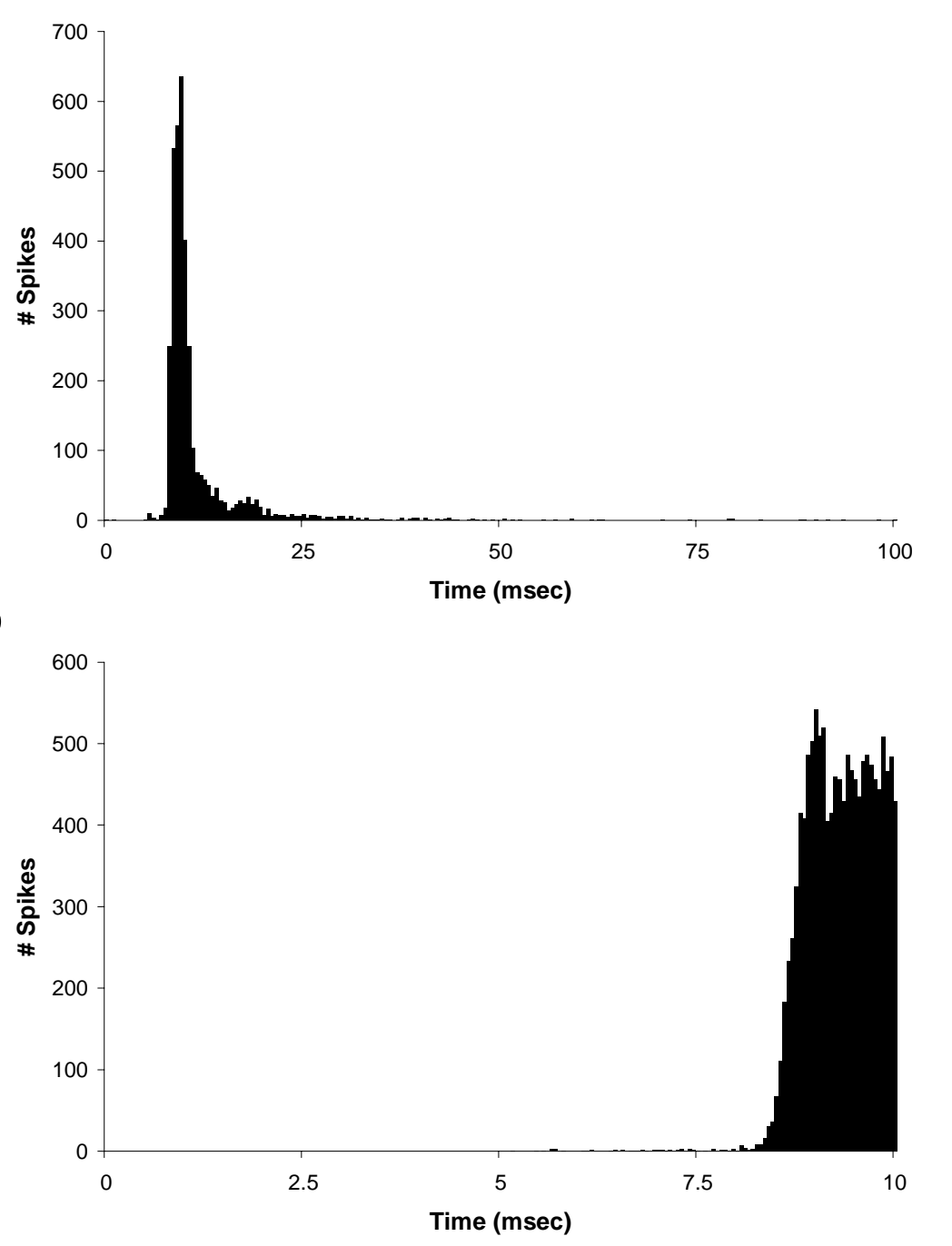

Fig. 14 Phase histograms of DHC responses used to generate autocorrelograms in fig. 15. 
A

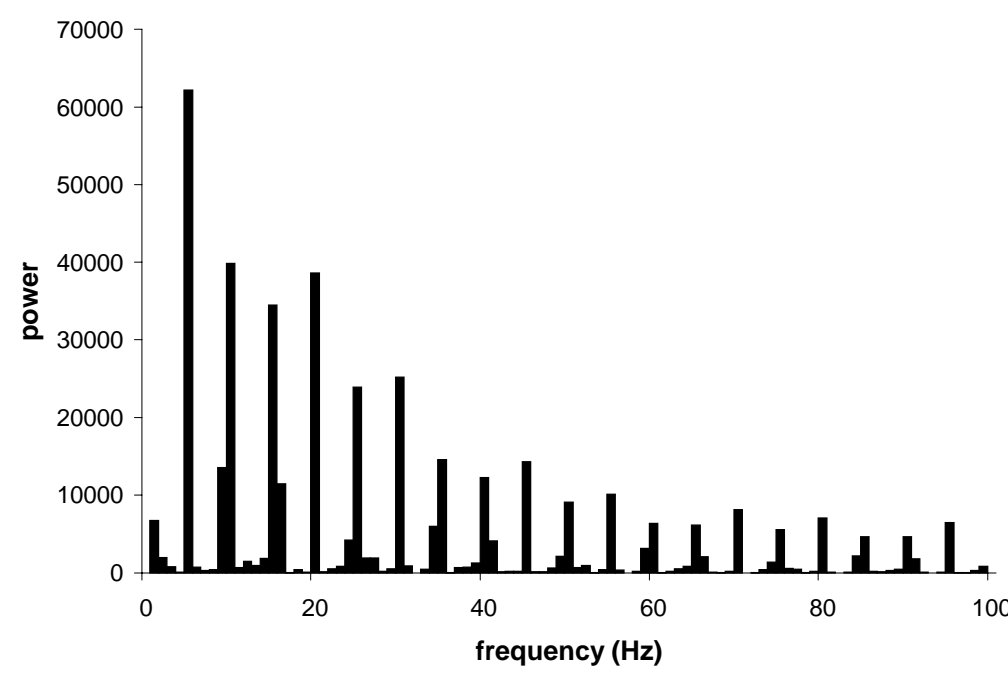

C

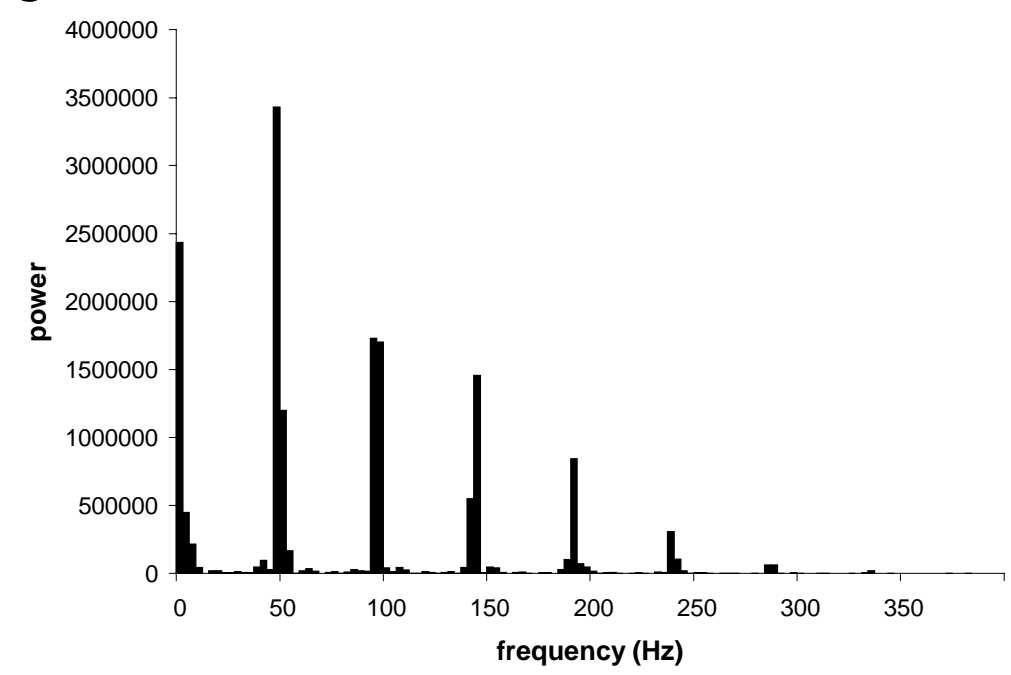

B

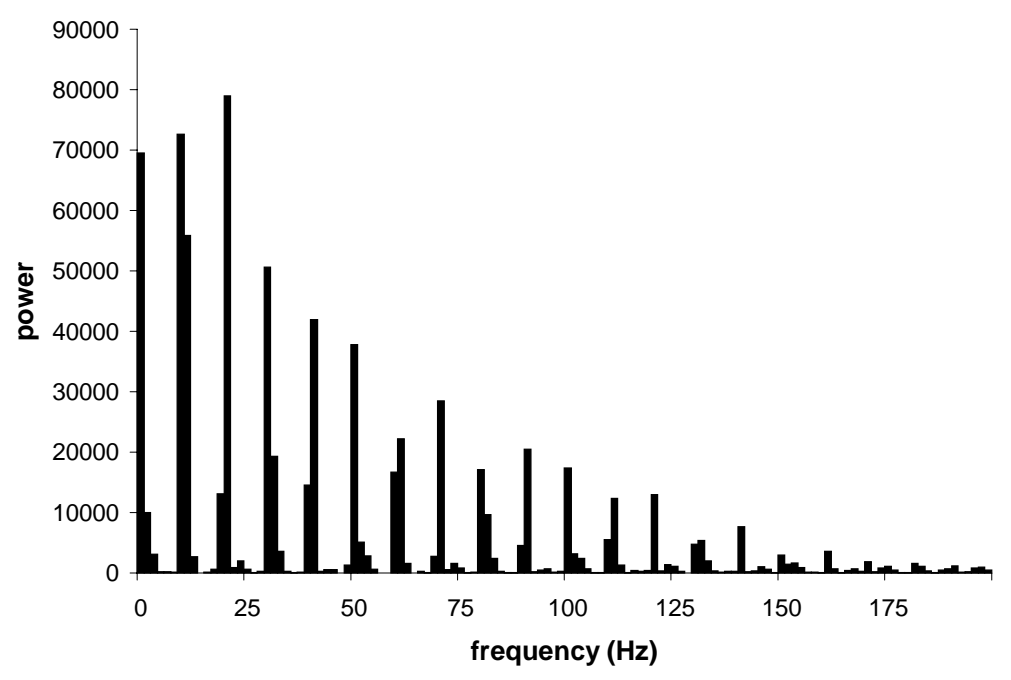

D

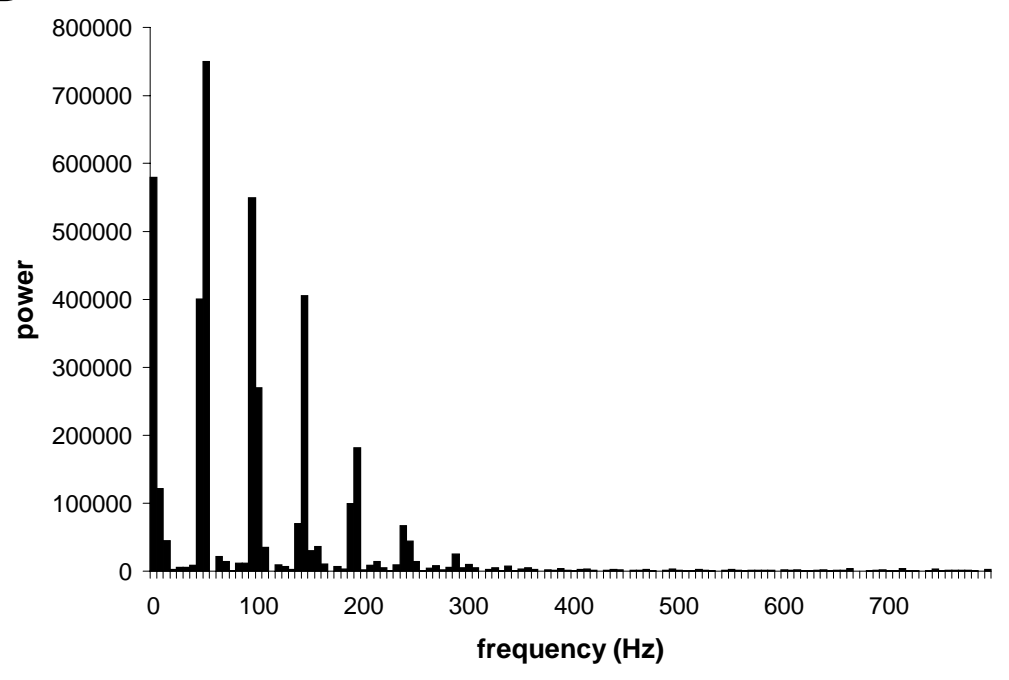

Fig. 15 Power spectra for autocorrelograms of cell responses in fig. 14. A-D, $5,10,50$ and $100 \mathrm{~s}^{-1}$ stimulation. 


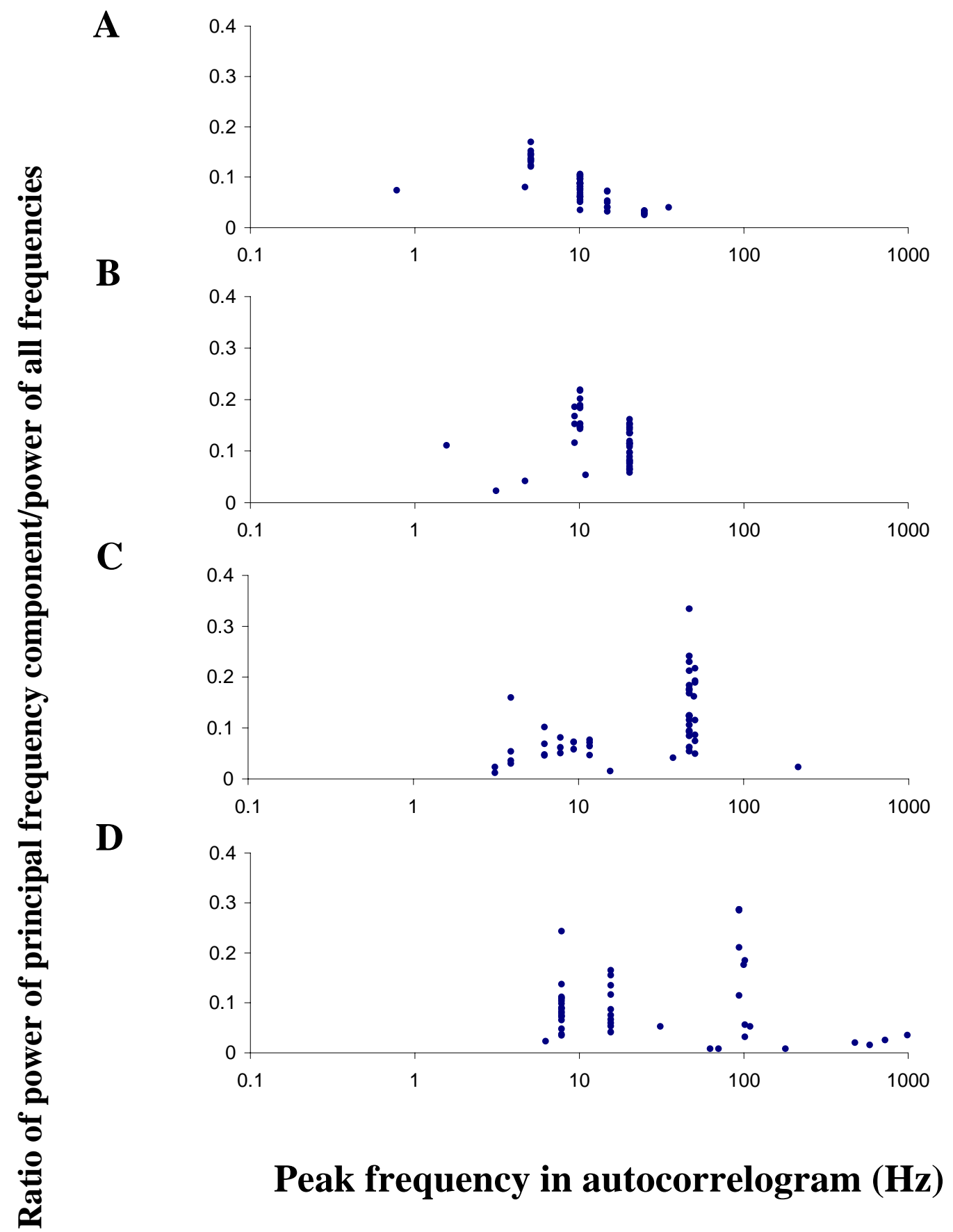

Fig. 16 Magnitude and frequency of principal frequency components of all cells' responses during (A-D) 5, 10, 50 and $100 \mathrm{~s}^{-1}$ stimulation. 


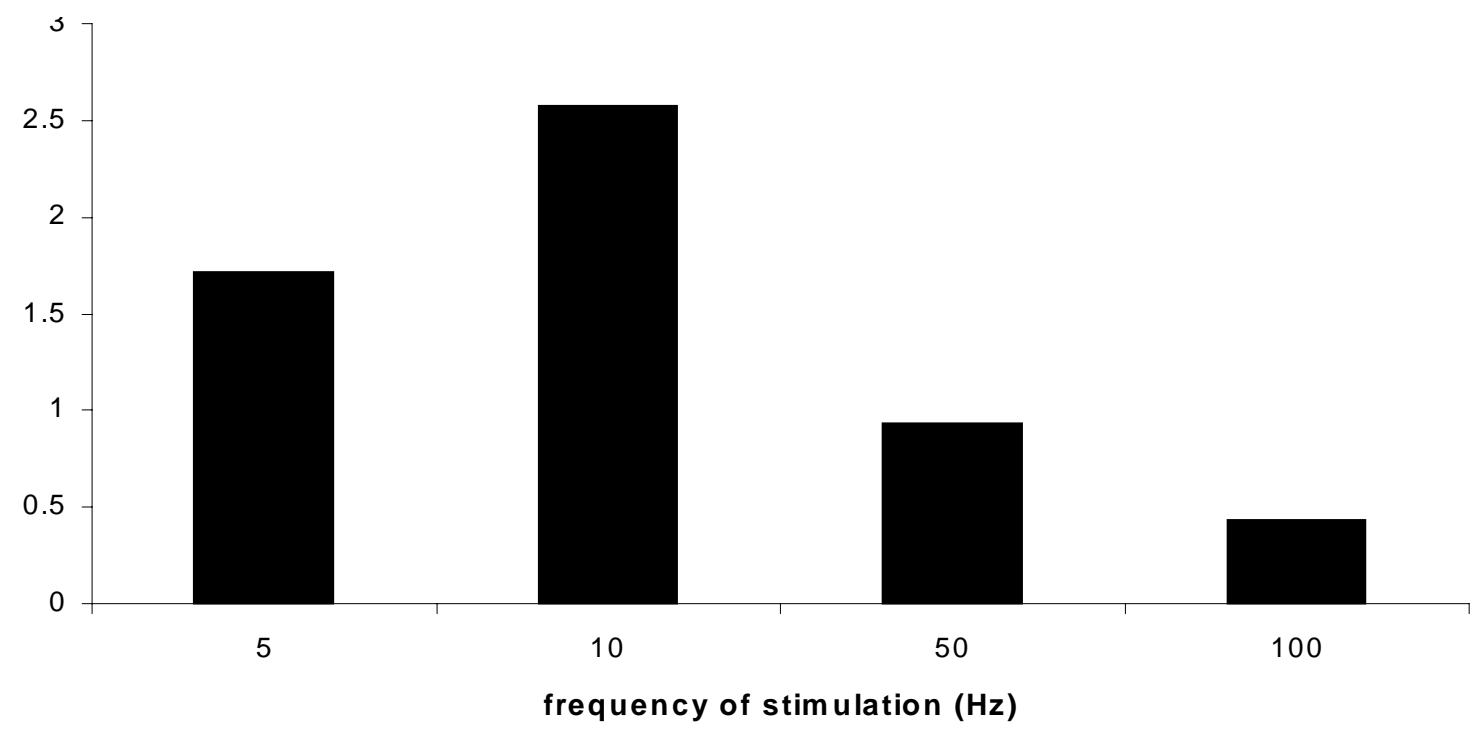

Fig. 17 Coefficients of variation for principal frequency components in the responses to electrical stimuli at four rates.

\section{Analysis of firing rates}

Distributions of firing rates for all cells during the full 100 seconds of stimulation at four stimulus rates are shown in fig. 18 Generally, firing rates increased when the stimulus frequency was increased from 5 to $10 \mathrm{~s}^{-1}$, and then rates displayed varying trends from 10 to $100 \mathrm{~s}^{-1}$. The trends for all cells are shown in fig. 19. The heavy broken line is the trend for all cells (population mean firing rates). There is not a uniform firing rate response to increasing stimulus frequency. Some cells increase, others decrease, still others do a combination of the two depending on the frequency of stimulation. The population mean firing rate increases with increasing stimulus frequency. The mean firing rates for all four frequencies of electrical stimulation are 
A

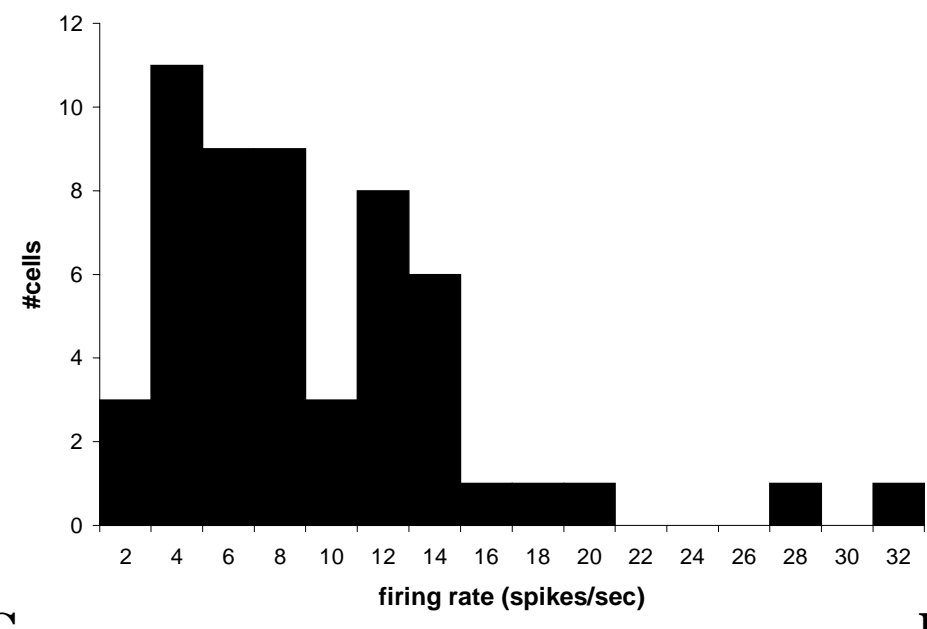

C

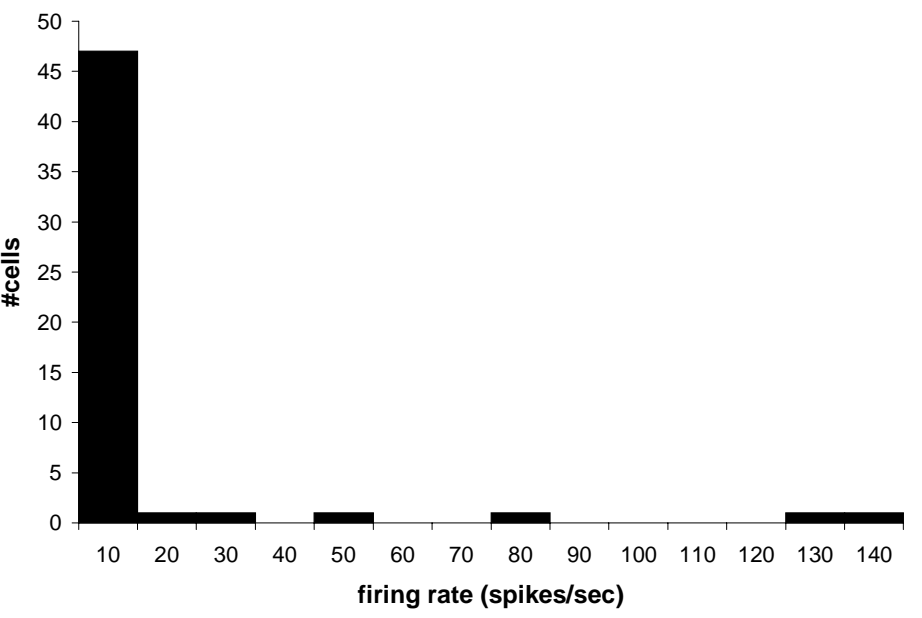

B

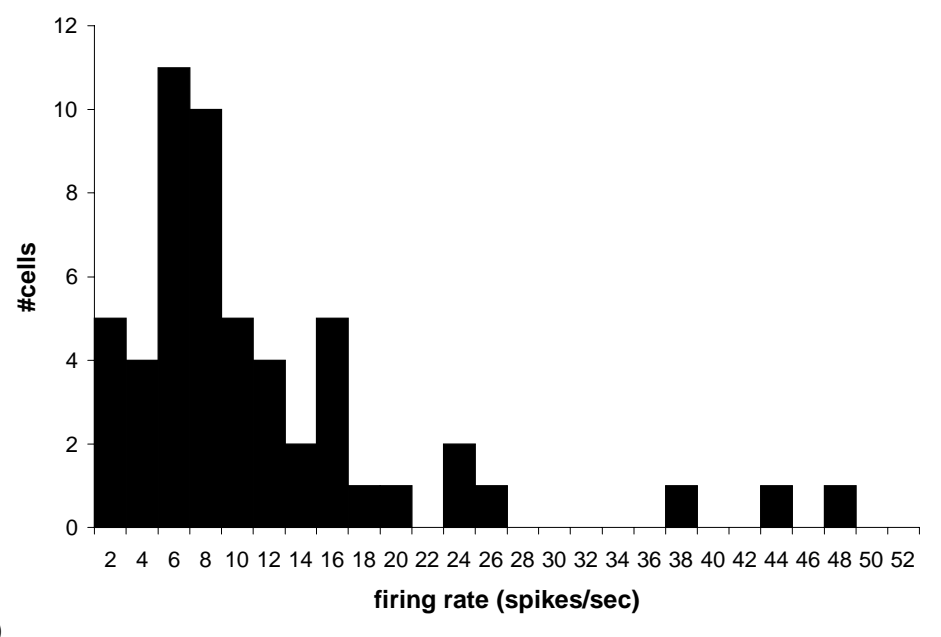

D

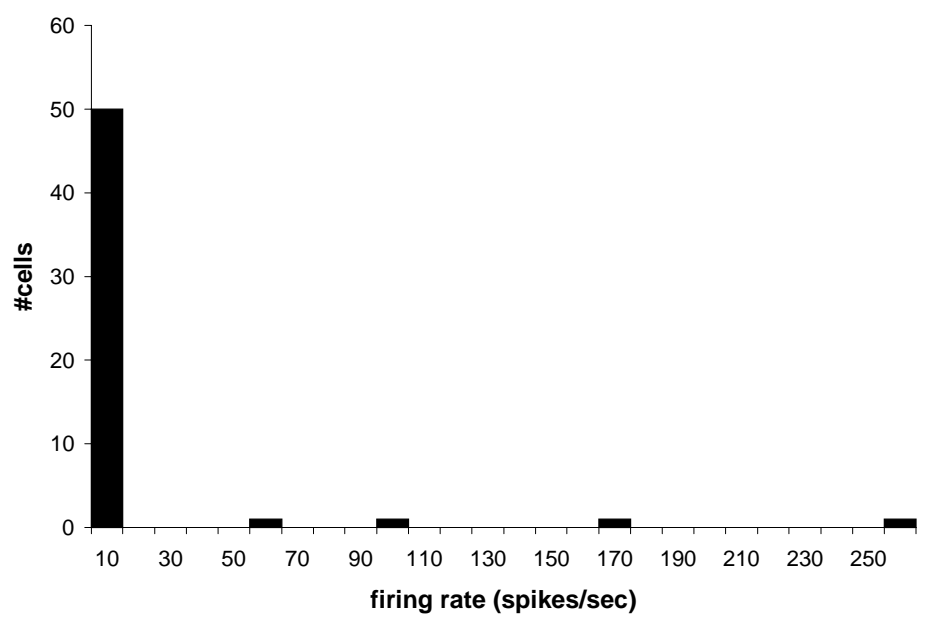

Fig. 18 Summary histograms of firing rates of DHCs during (A-D) 5, 10, 50 and $100 \mathrm{~s}^{-}$ ${ }^{1}$ stimulation. 


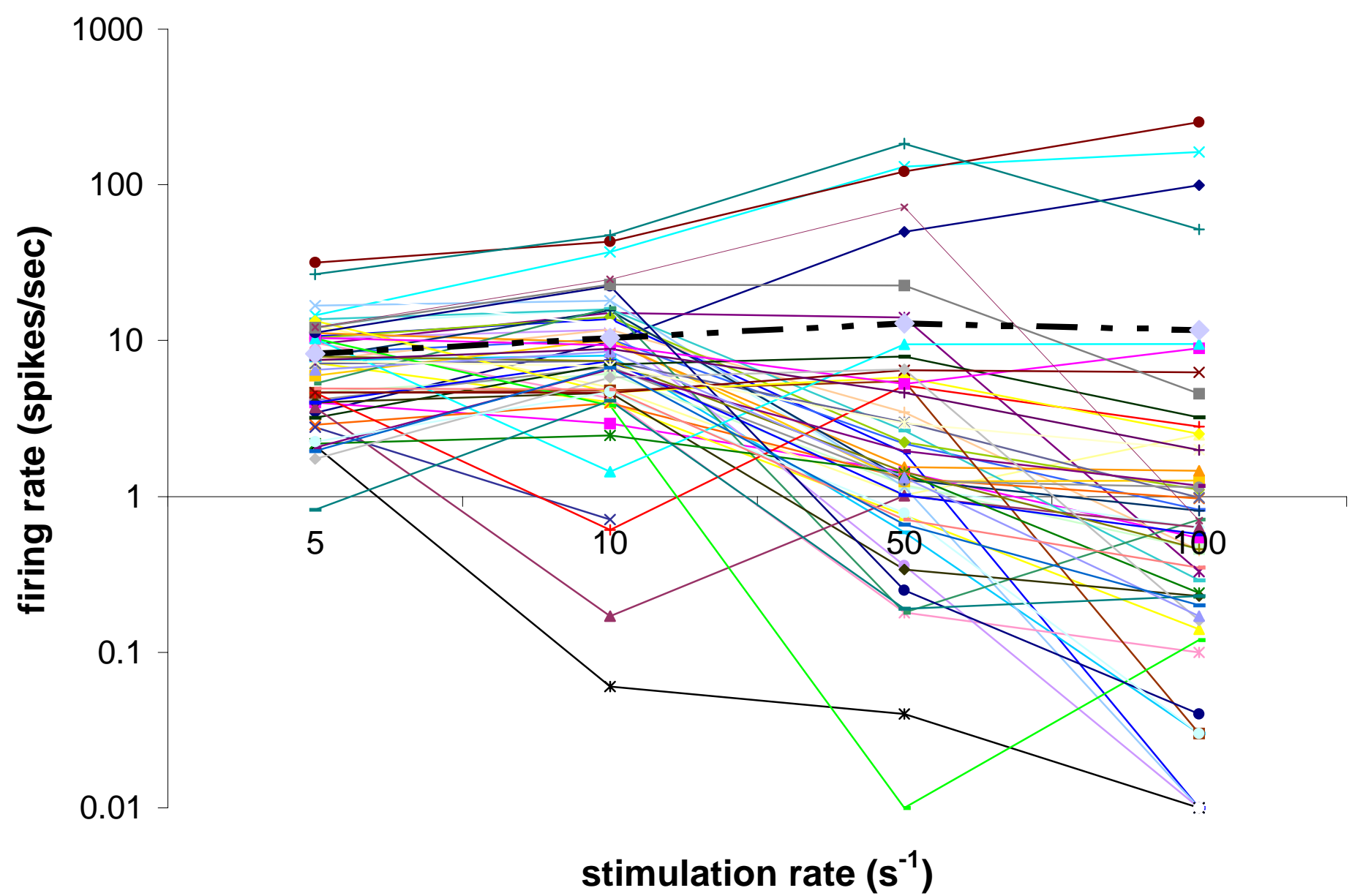

Fig. 19. Firing rates of all DHCs during 4 rates of stimulation. Dark hatched line reflects trend for all cells. 
shown in fig. 20. The standard error bars clearly show that there is no significant trend in firing rate changes.

Some DHCs respond with bursts of action potentials when their RFs are stimulated with single pulsatile stimuli. Cells that responded at a rate higher than the frequency of stimulation were firing more than once per stimulus, on average. The number of cells that exhibited this type of behavior is shown in fig. 21. While a large percentage of the cells respond in a bursting fashion at stimulation frequencies of 5 and $10 \mathrm{~s}^{-1}$ (60 and $34 \%$ respectively), very few (5\% or less) respond in this way at 50 and $100 \mathrm{~s}^{-1}$. This is probably due to fatigue in synaptic transmission, or failure of the afferent fibers to encode high stimulus rates.

Phase vector magnitude was plotted vs. firing rate in fig. 22. The magnitude of the phase vector and the firing rate are not well correlated. In fact, cells with higher firing rates generally have lower phase vector magnitudes. This isn't surprising considering the fact that increasing numbers of spikes add more unit components to the phase vector for a cell's responses, and they will tend to drive the magnitude of the phase vector down. The greater the number of spikes, the more likely it is that the timing of events will be more asynchronous, if the firing rate is greater than 1 spike/cycle. 


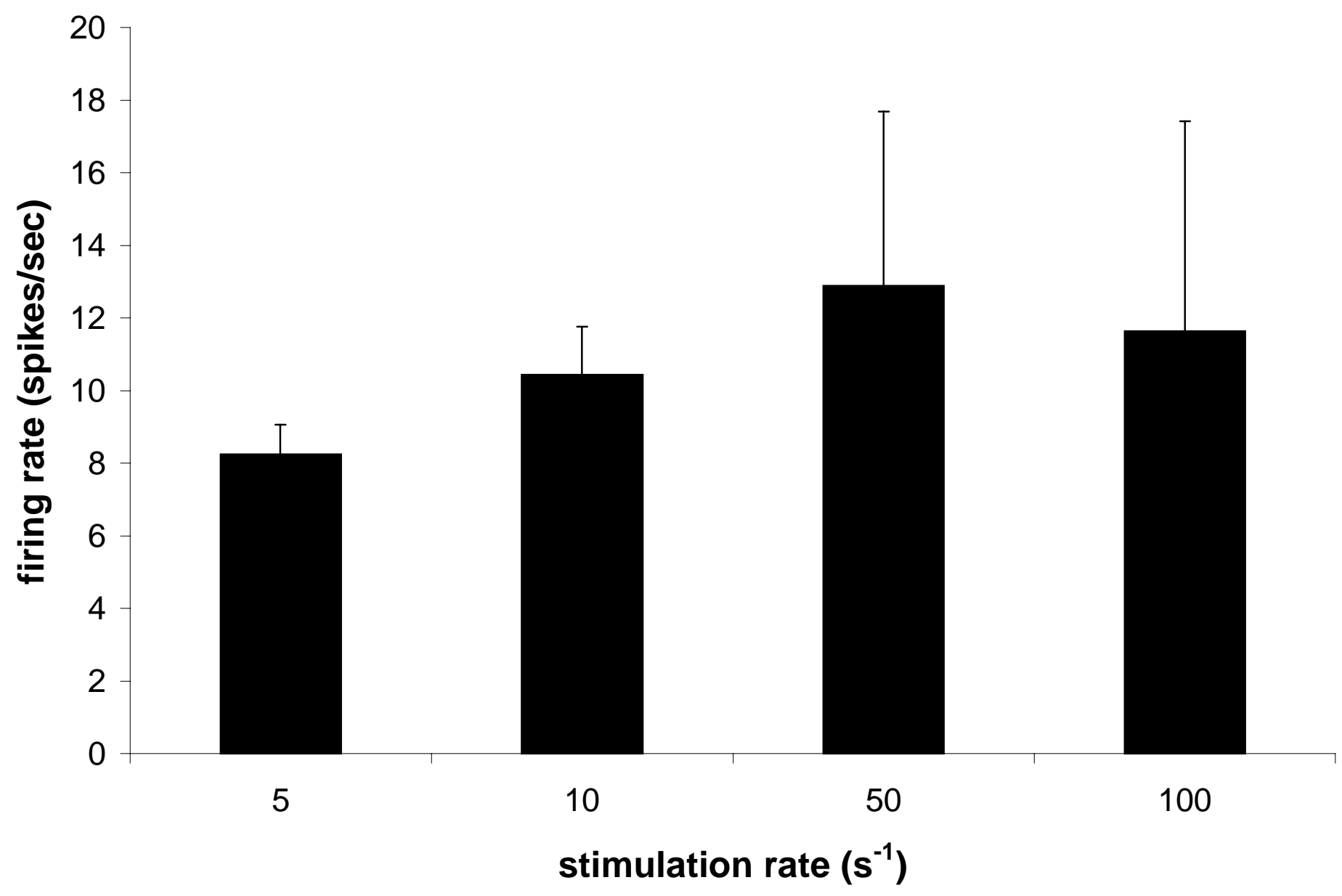

Fig. 20. Mean firing rates of DHCs at 4 stimulation rates. 


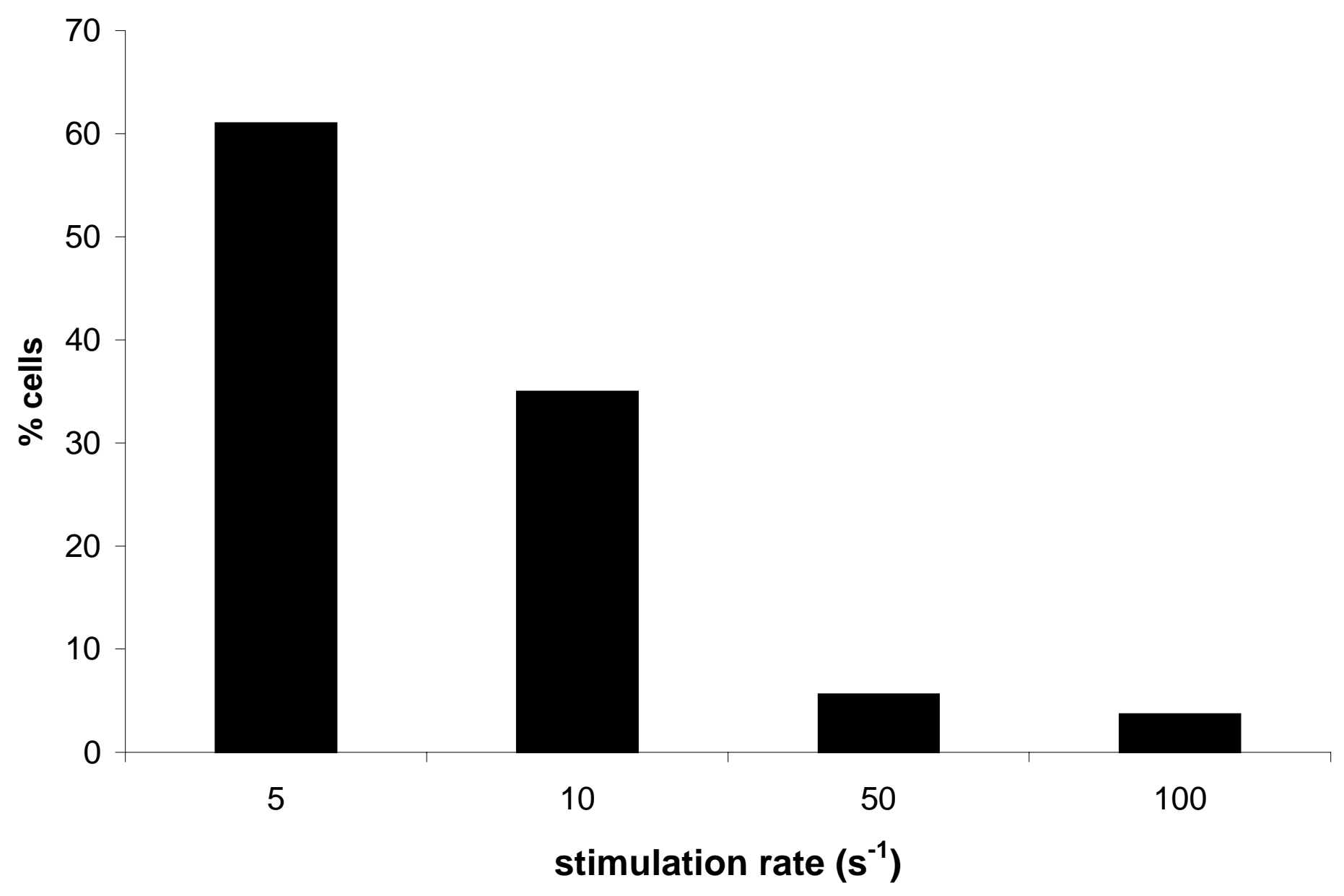

Fig. 21. Percentage of cells with firing rates greater than stimulation rates. 

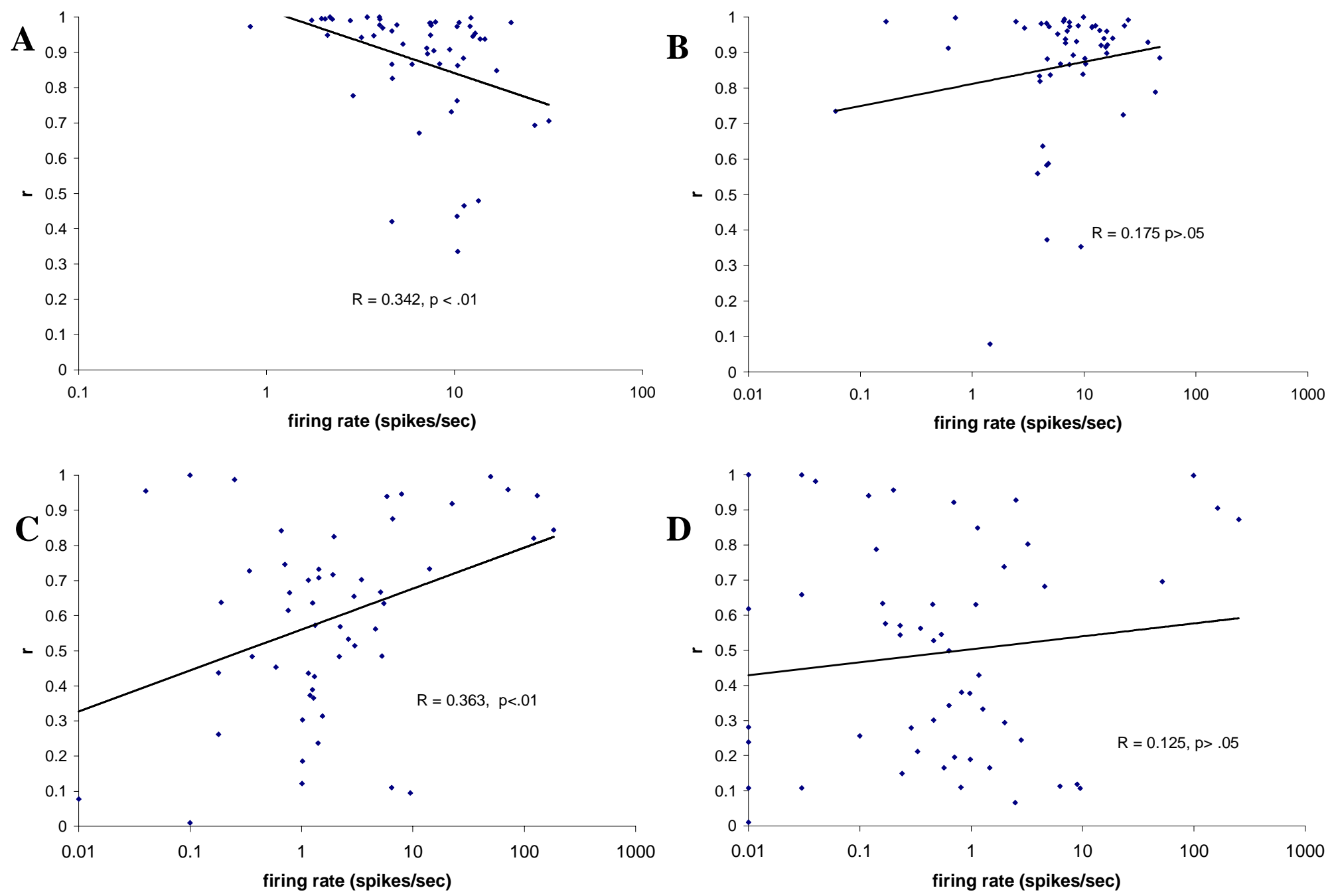

Fig. 22. Phase vector strength vs. firing rate during (A-D) $5,10,50$ and $100 \mathrm{~s}^{-1} \mathrm{~s}$ stimulation. 


\section{Population responses}

The autocorrelograms for the combined responses of all DHCs are found in fig. 23. The amalgamate cell responses show a clear periodicity coinciding with the periodicity of the stimulus. Fig. 24 shows the power spectra for the population response. The principal frequency component for each spectrum falls at or near the corresponding frequency of stimulation. This is interesting considering the fact that no correction is made for the response latencies of each cell due to conduction distance and velocity differences. This effect would be at a maximum where stimulus periods are short, but that is probably compensated by the fact that few cells produced many spikes at high stimulation rates. At the high stimulation rates, the responsive cells dominate the autocorrelogram, resulting in a principal frequency component at or near the frequency of stimulation. 
A

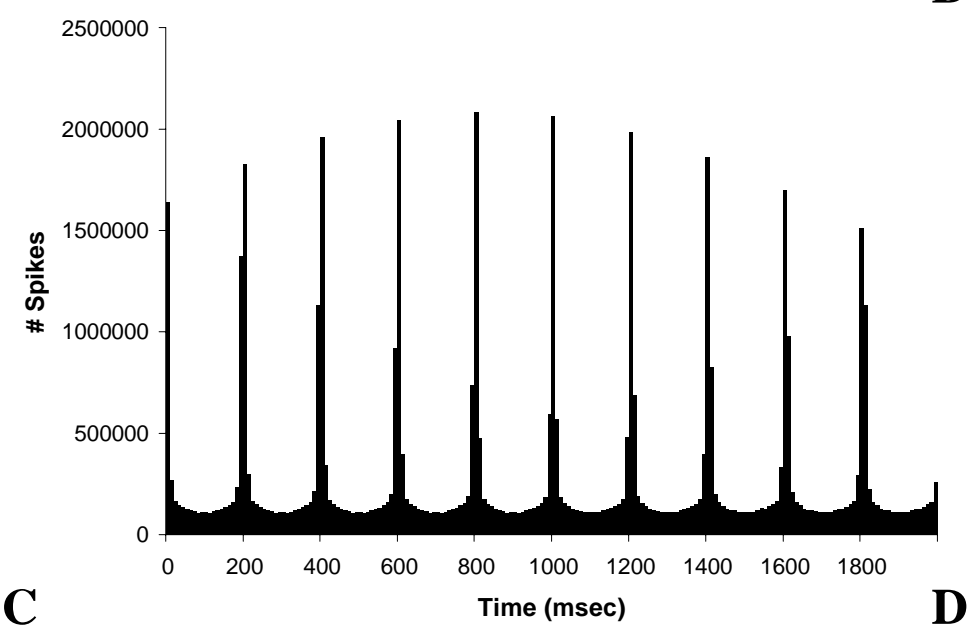

B
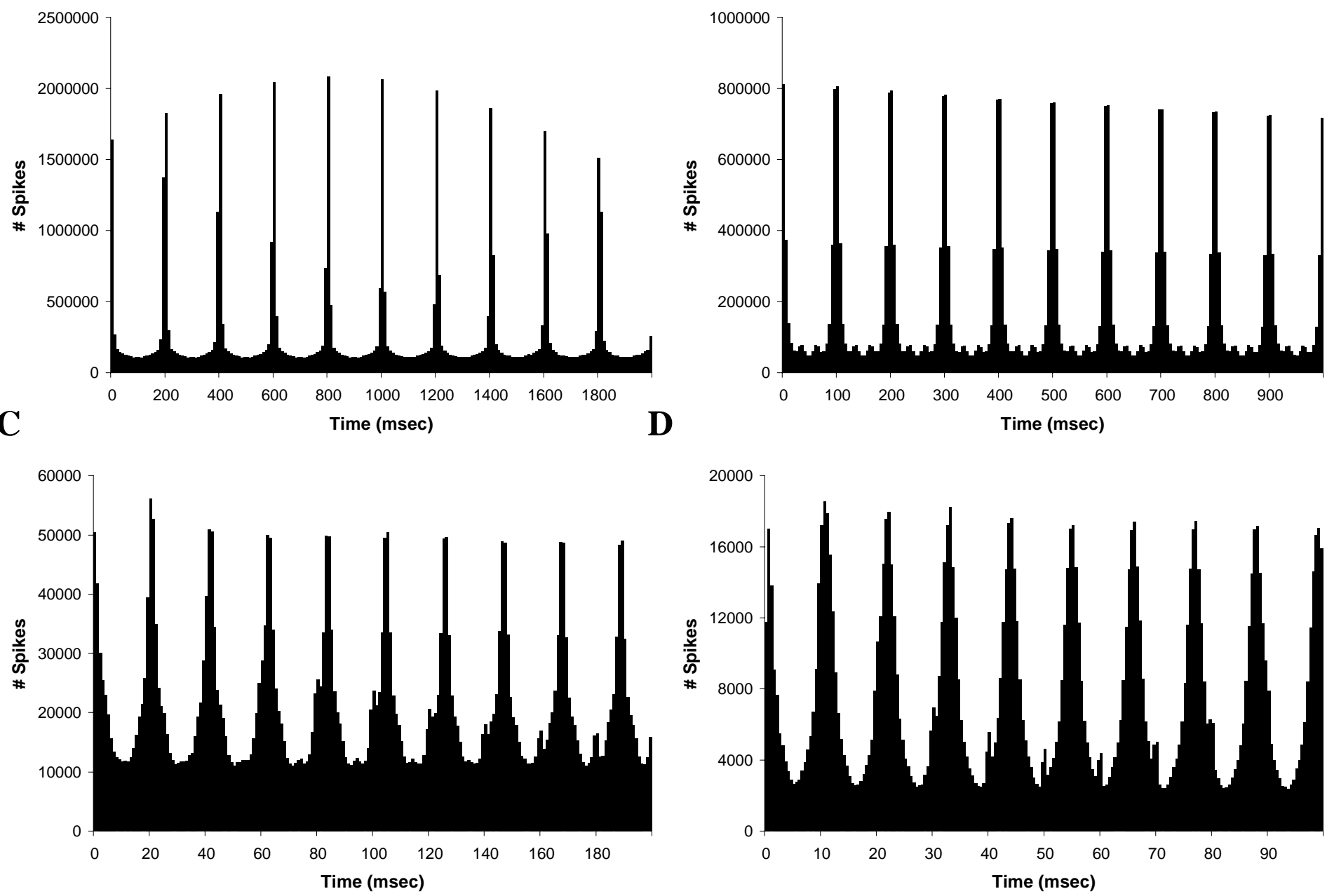

Fig. 23. Autocorrelograms for DHC population responses to four rates of electrical stimulation. A-D, 5, 10, 50 and $100 \mathrm{~s}^{-1}$ 
A

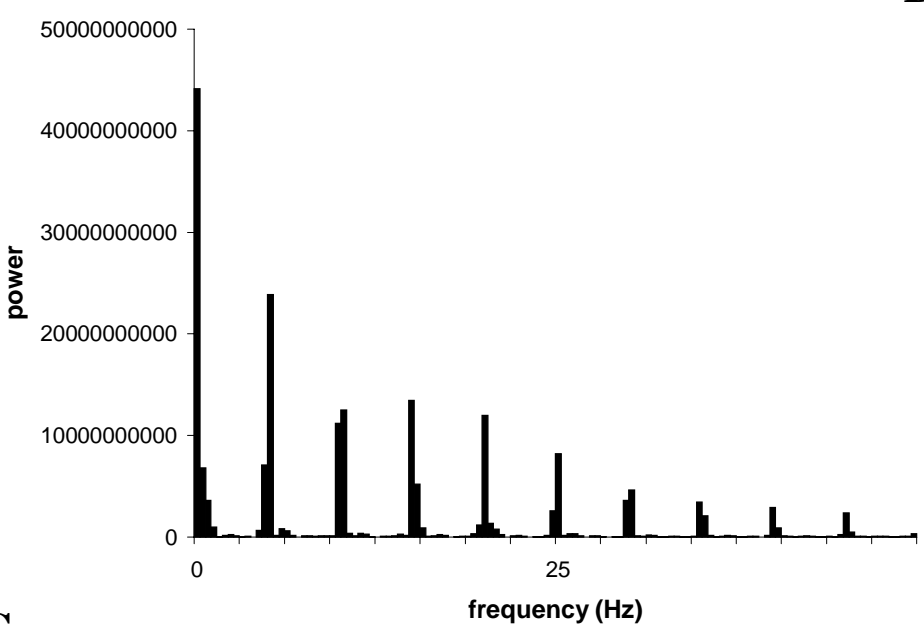

C

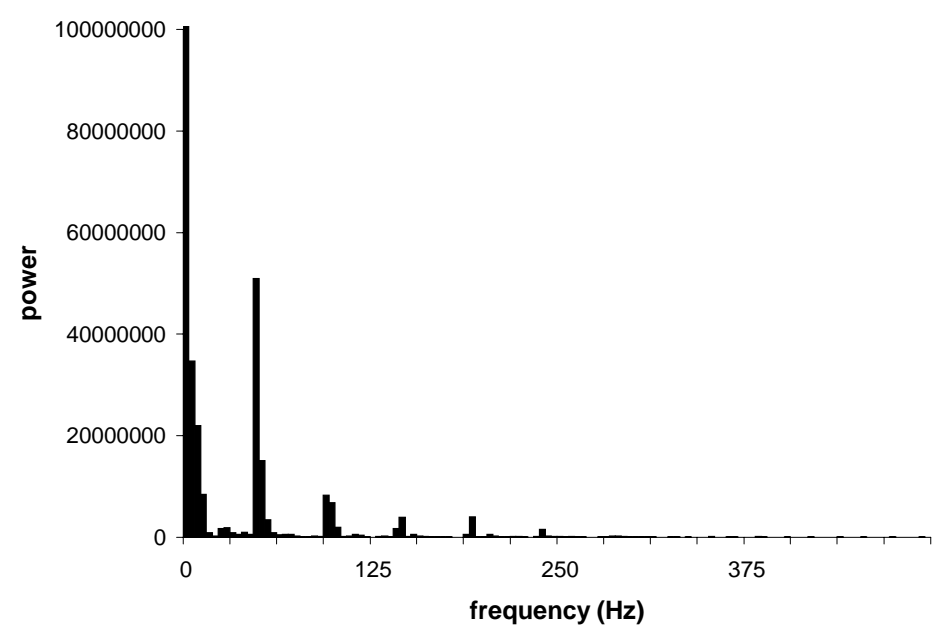

B

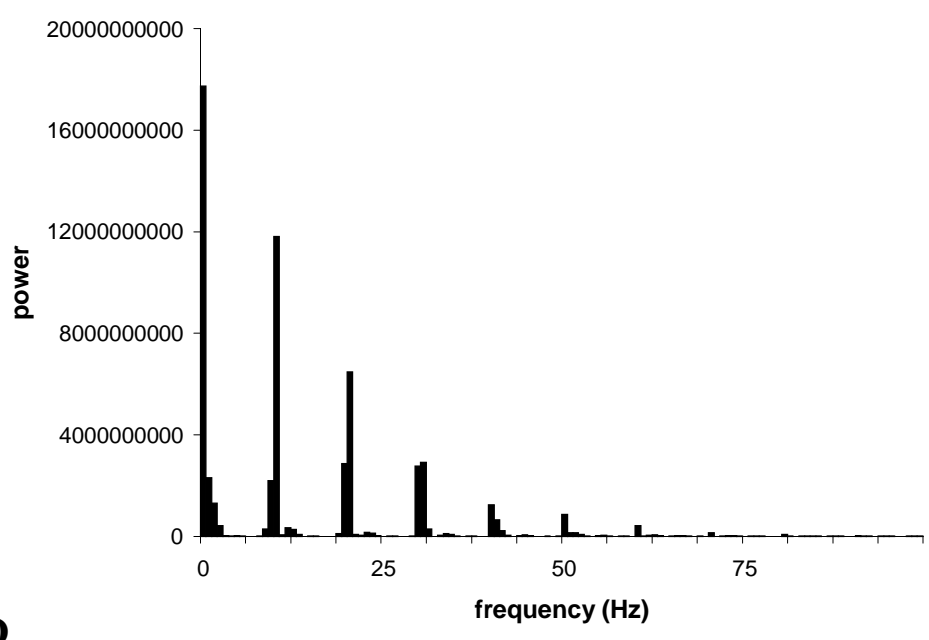

D

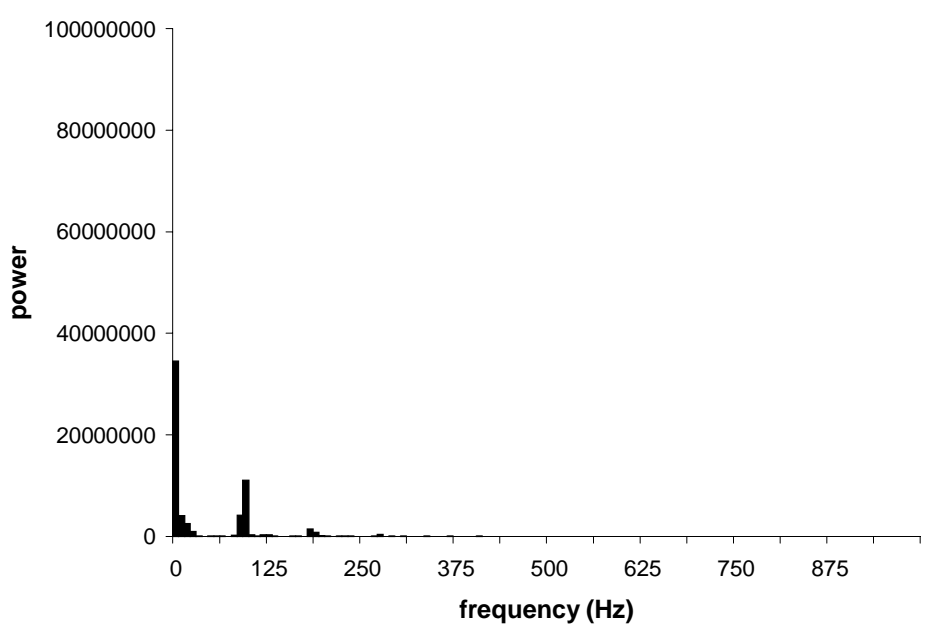

Fig. 24. Power spectra for $\mathrm{DHC}$ population responses to four rates of electrical stimulation. A-D, 5, 10, 50 and $100 \mathrm{~s}^{-1}$. 


\section{Effects of adaptation}

Phase vector and firing rate analyses were performed on the responses of DHCs during the first ten stimuli at each frequency. Fig. 25 shows the distribution of phase vectors at four frequencies of stimulation. Phase vector magnitudes are larger for 10 stimulus presentations than for the 10 second presentations. This indicates that there is some effect of adaptation on phase locking, and the cells are much better at phase locking during the initial part of the stimulus. Fig. 26 shows the distribution of all vector strengths for responses to 10 cycles of electrical stimuli presented at 4 frequencies. Mean phase vectors and standard errors are shown in fig. 27. There are a number of cells that have no phase vector at all, since no responses fell within the period of the first ten cycles of stimulation. This is particularly true of the 50 and $100 \mathrm{~s}^{-1}$ stimuli, where 10 stimuli accounts for 200 and $100 \mathrm{msec}$ of response time, respectively. The probability of a response occurring in the first ten presentations of a stimulus goes down with increasing stimulus rate.

The firing rates of DHCs during the first 10 cycles of stimulation are shown in fig. 28. The rates are higher for the responses to 10 stimuli than for the full ten seconds of stimulus time mean firing rates are higher than those for the full 10 seconds of stimulation. They also have greater variability, which can be seen in the error bars at 50 and $100 \mathrm{~s}^{-1}$ in fig. 29. The standard errors are slightly higher than for the ten second responses. 
A

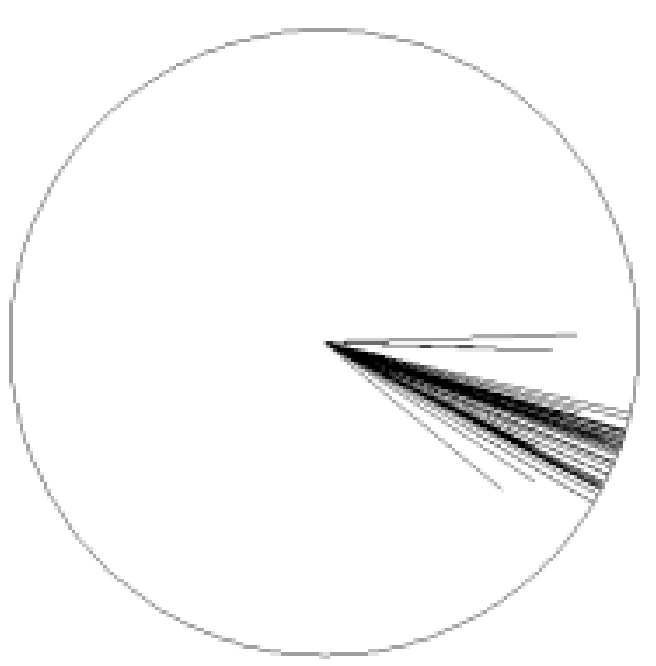

C

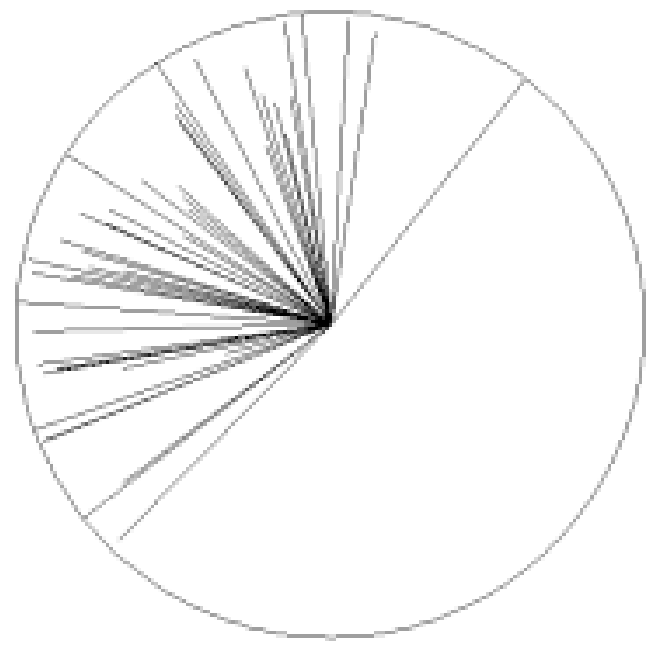

B

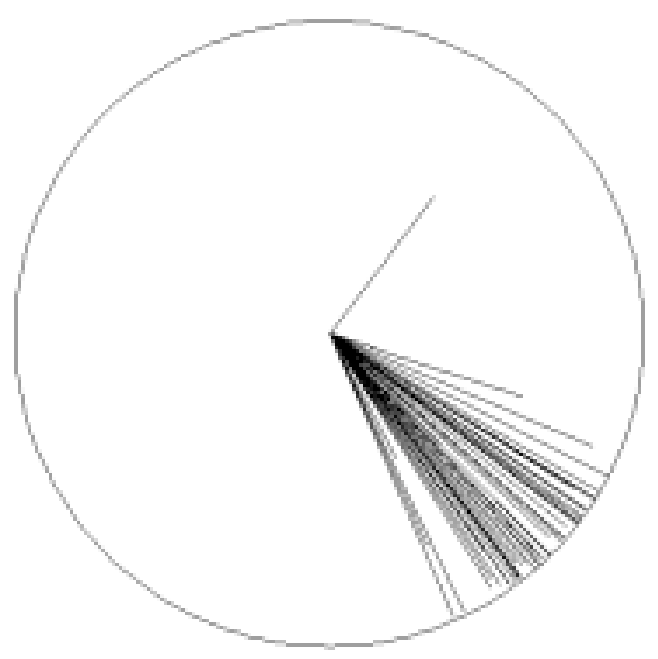

D

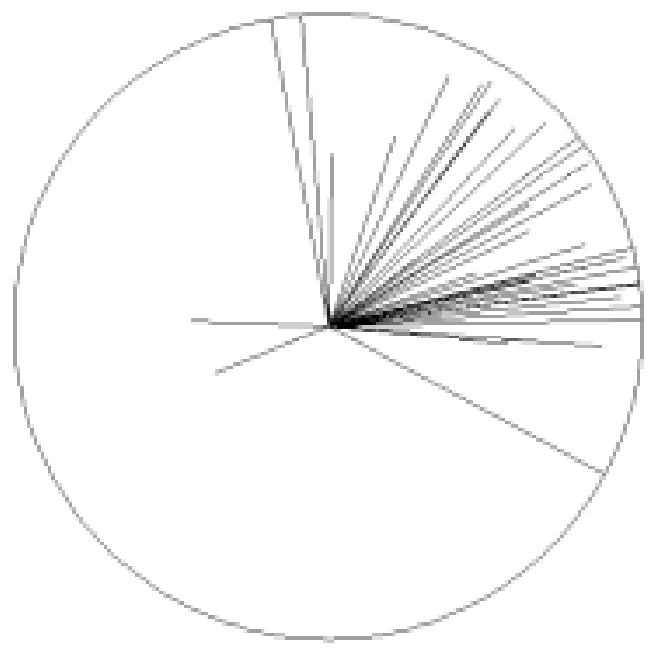

Fig. 25. Phase vectors for all DHCs for the first ten stimuli of each stimulus trial. A-D, $5,10,50$ and $100 \mathrm{~s}^{-1}$ stimulation. 
A

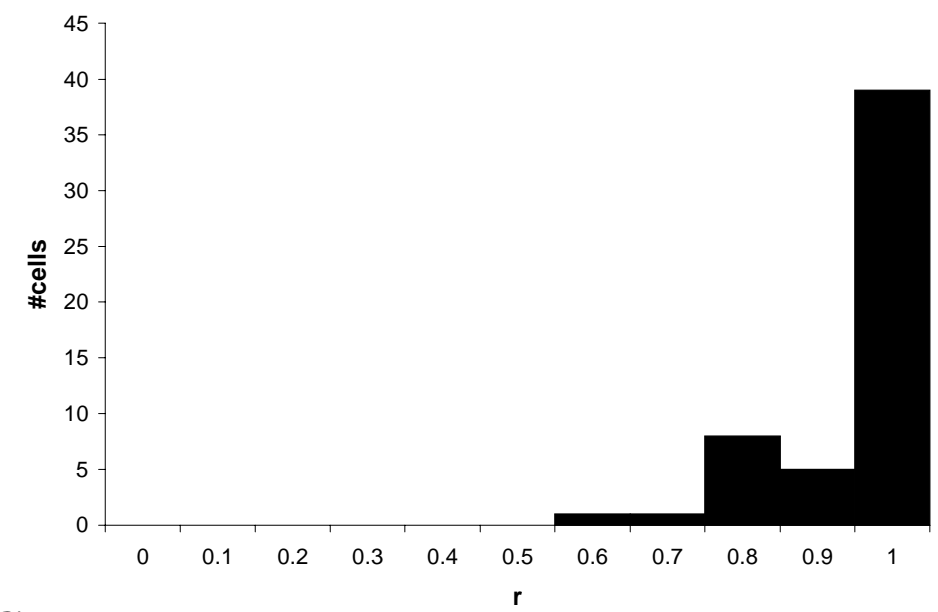

C

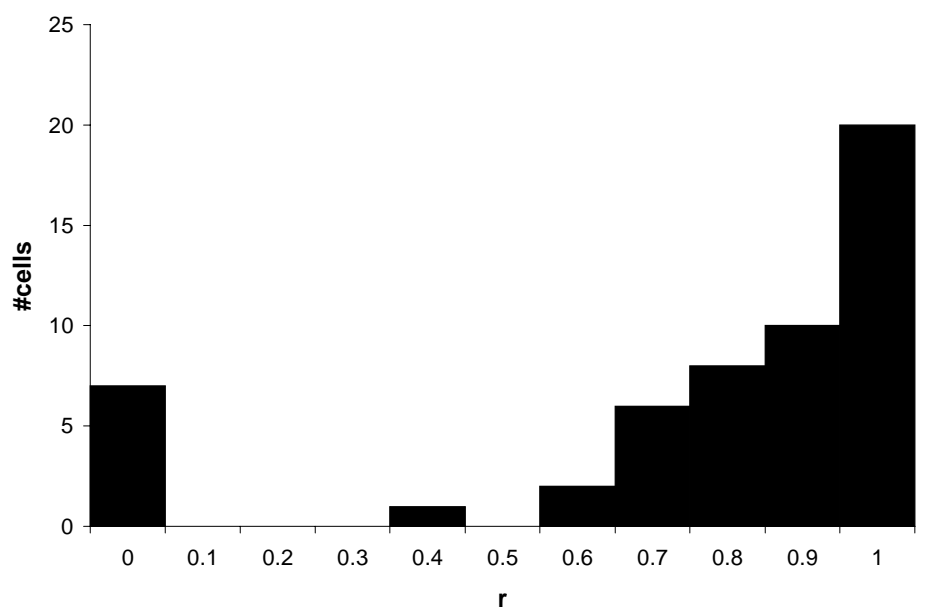

B

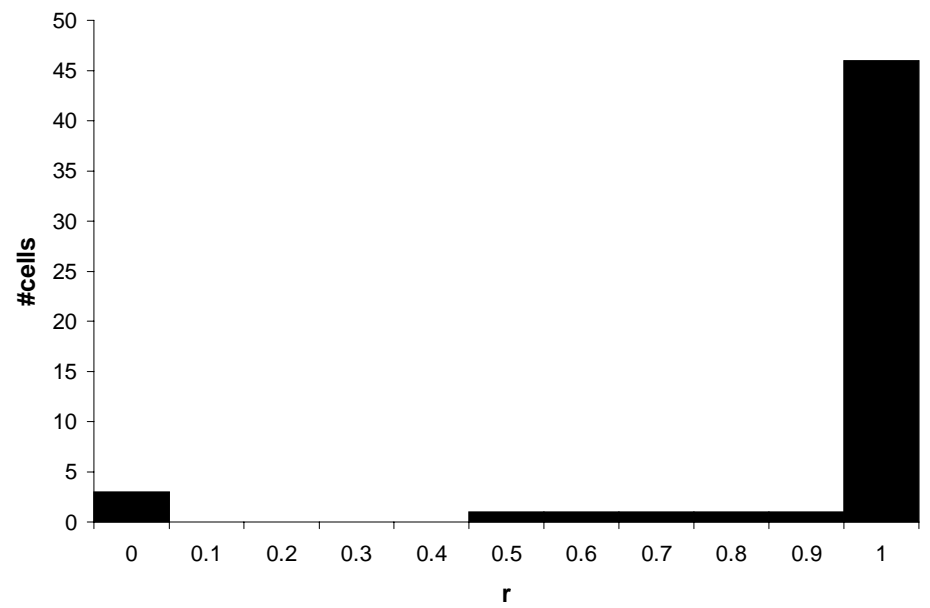

D

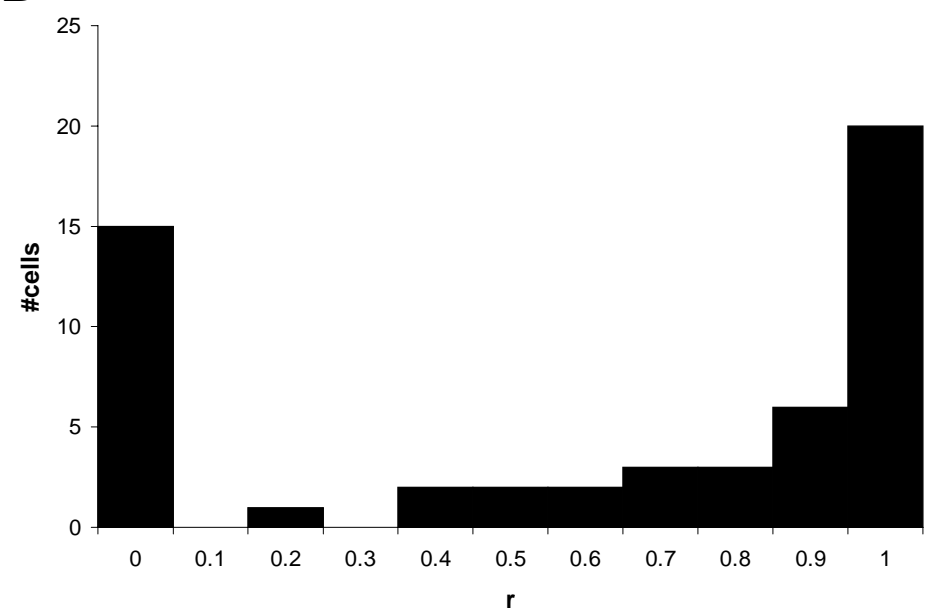

Fig. 26. Summary histograms of vector strengths for $\mathrm{DHC}$ responses during the first ten stimuli of each stimulus trial for (A-D) $5,10,50$ and $100 \mathrm{~s}^{-1}$ 


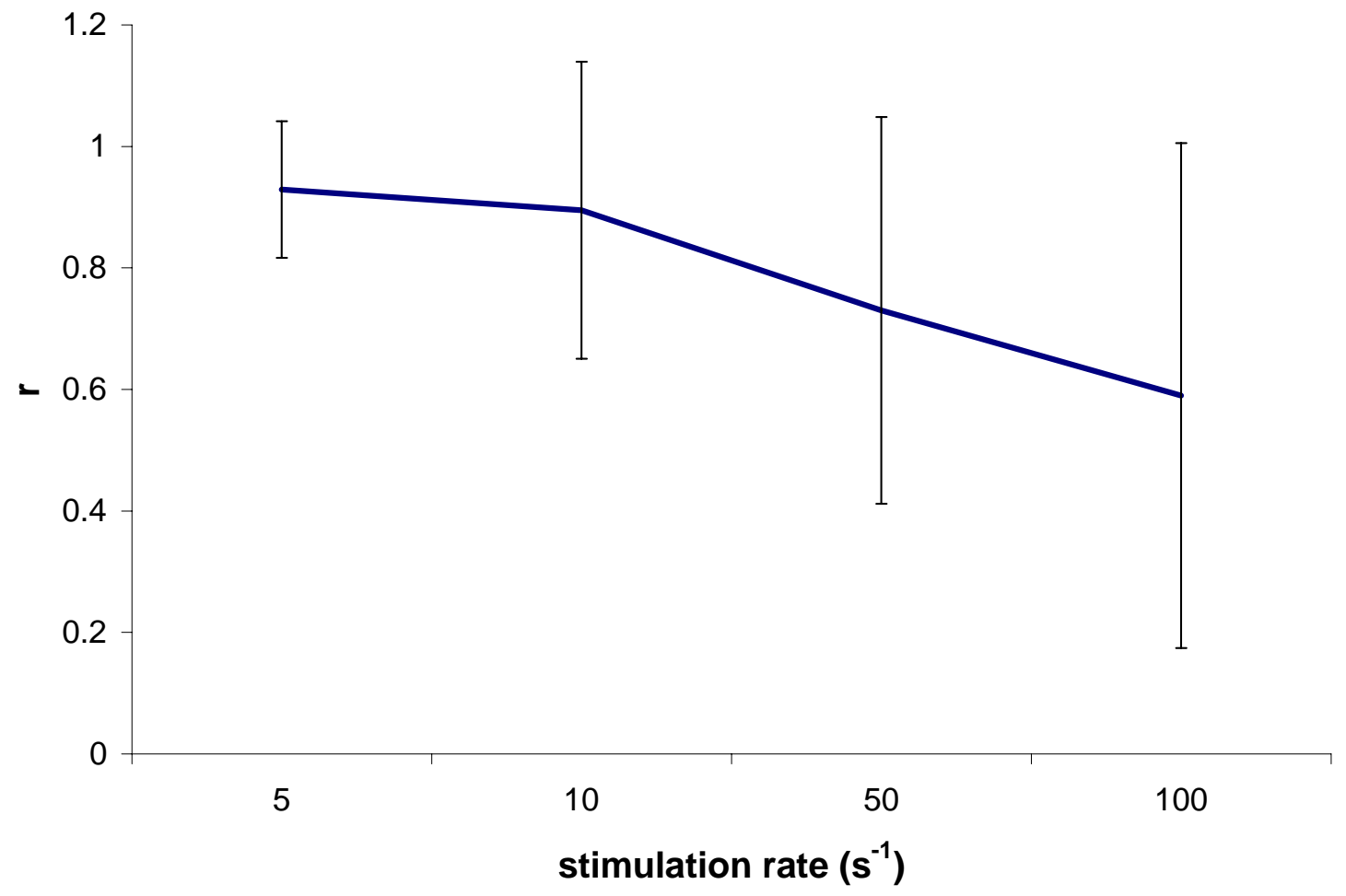

Fig. 27. Mean vector strengths for $\mathrm{DHC}$ responses during the first ten stimululi of each stimulus trial, for four stimulation rates. 
A

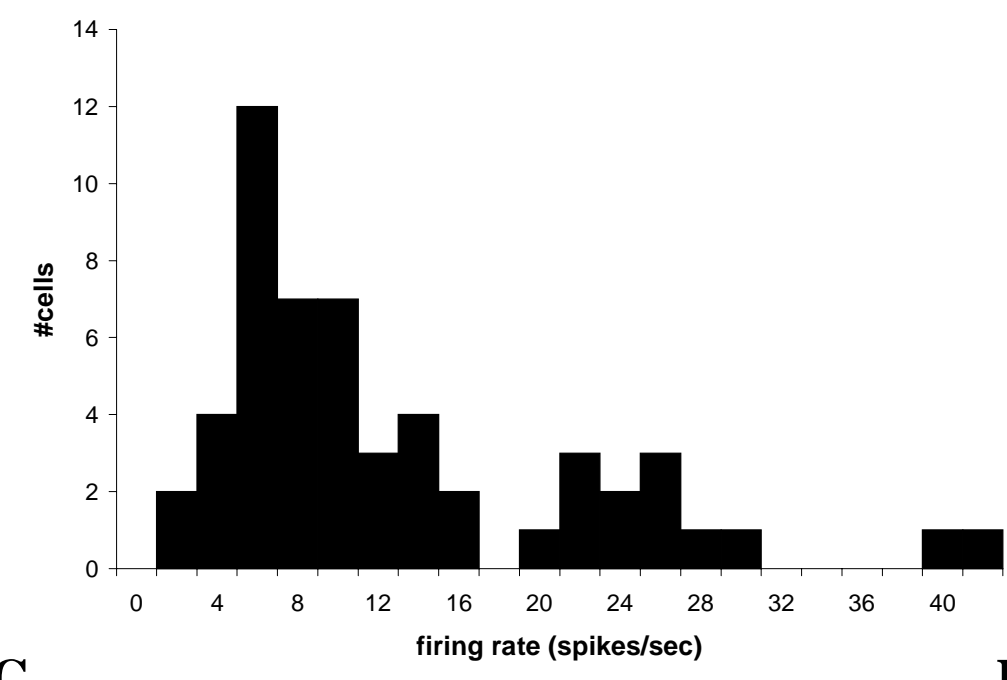

C

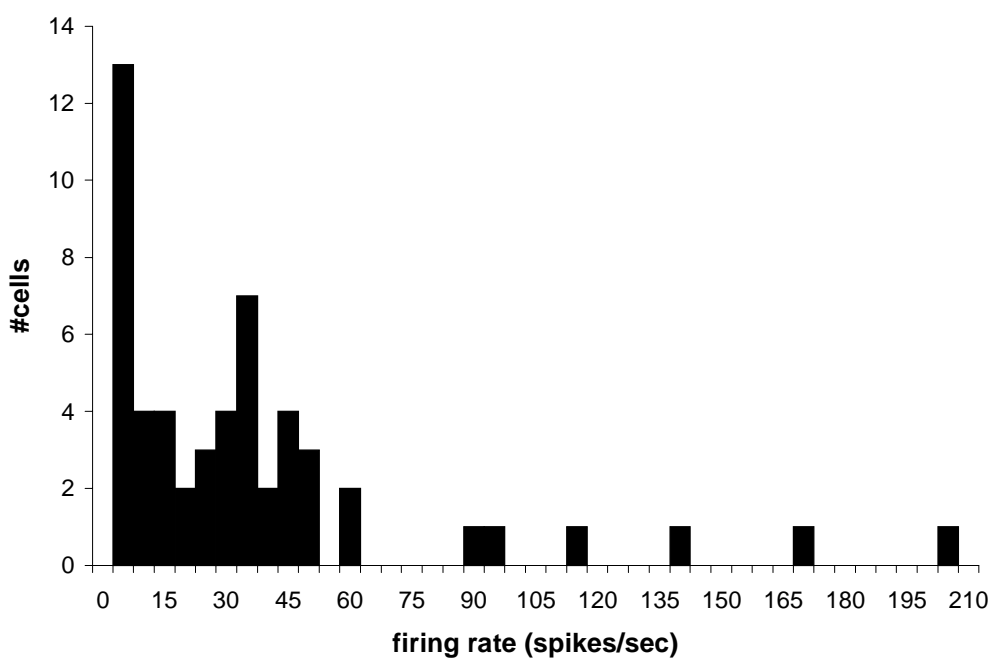

B

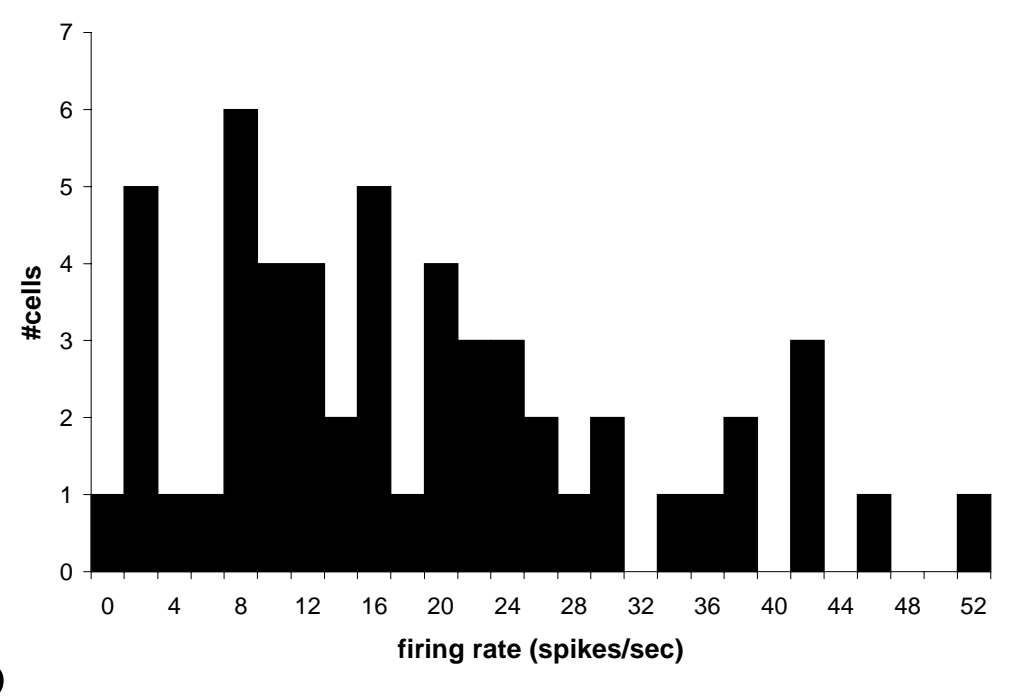

D

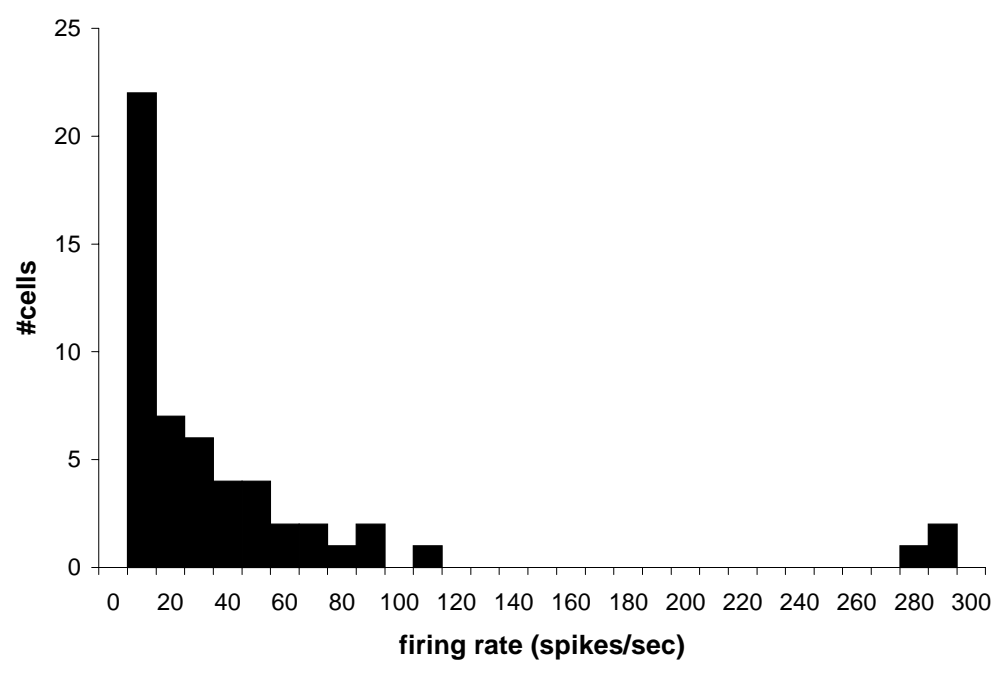

Fig. 28. Summary histograms of DHC firing rates for the first ten stimuli of each stimulus trial, at (A-D) 5, 10, 50 and $100 \mathrm{~s}^{-1}$ stimulation rates. 


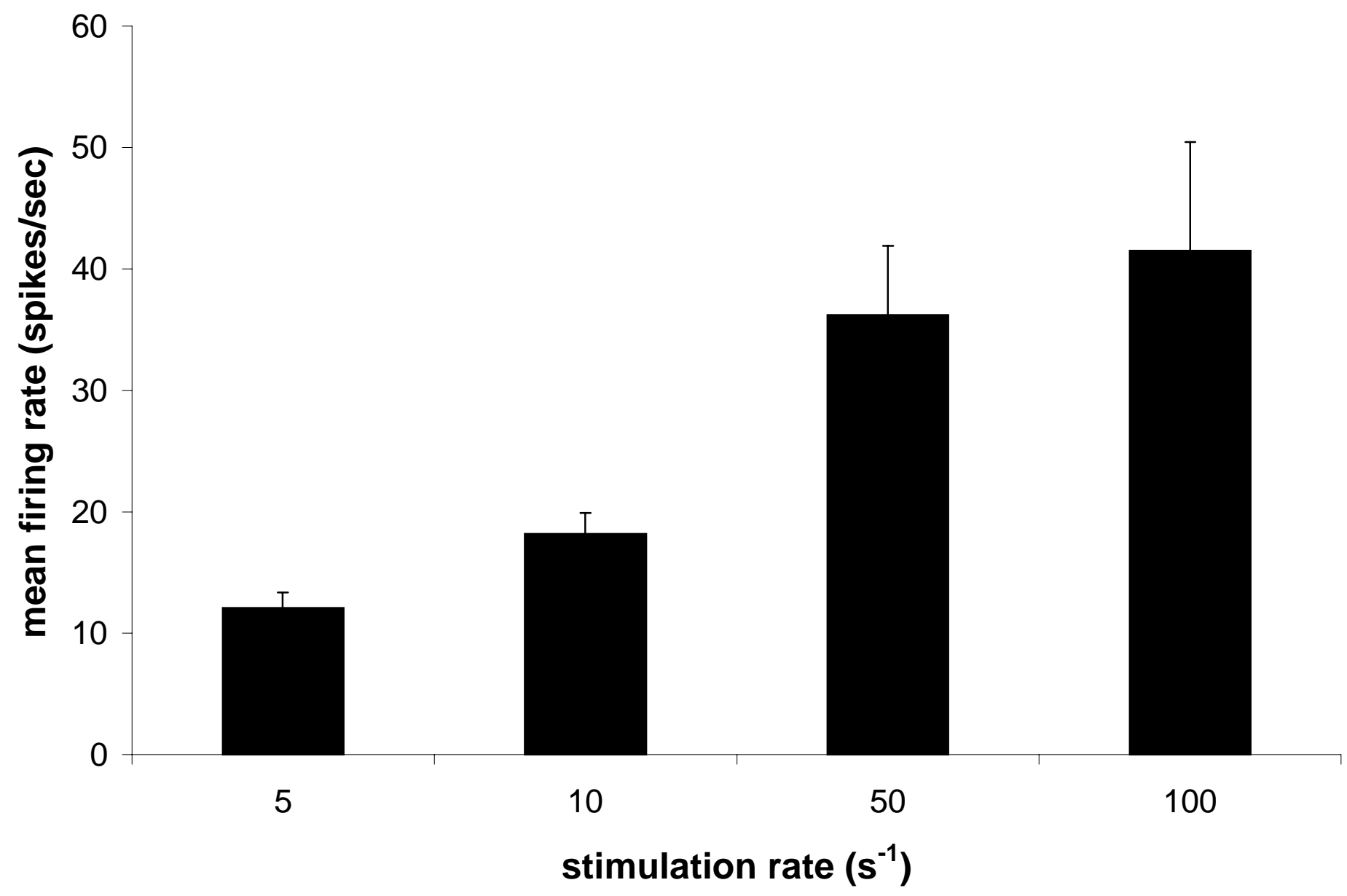

Fig. 29. Mean firing rates for DHCs during the first ten stumuli of each stimulus trial, at four stimulation rates. 


\section{B. Mechanical stimulation of DHC RFs}

Full characterizations of 24 cells were obtained in 4 animals. All 10 cells that were reconstructed were located in laminae III-IV. RFs are shown in fig. 30.

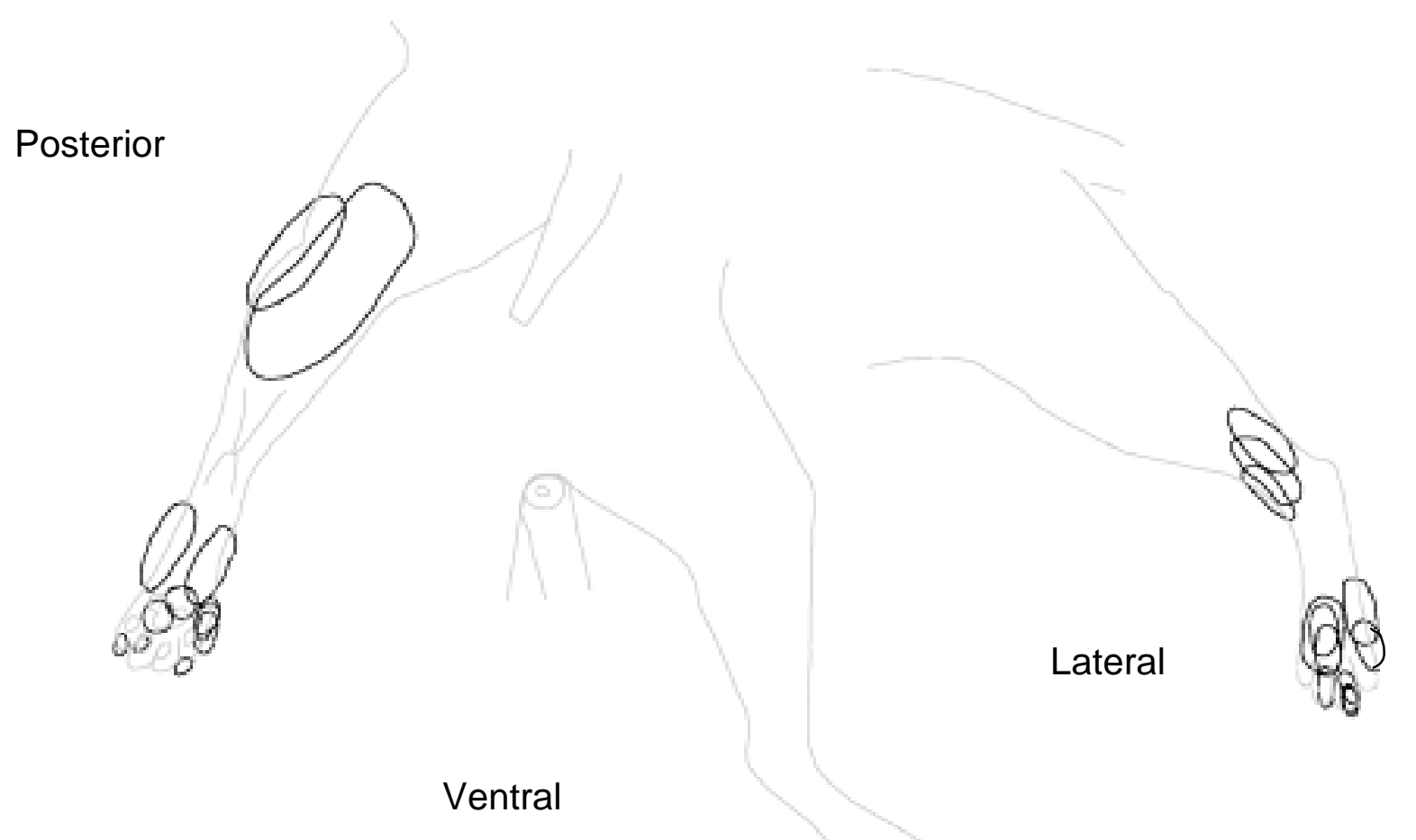

Fig. 30. RF locations of mechanically characterized cells.

\section{Histogram analysis}

PSTHs for two typical and one atypical cell are shown in figs. 31-35. PSTHs of responses during $5,10,50,100$, and $200 \mathrm{~Hz} 500 \mu \mathrm{m}(300 \mu \mathrm{m}$ for $200 \mathrm{~Hz})$ sinusoidal stimulation show the two broad classes of responses for the tested neurons. The 
highest amplitude of stimulation is shown since $\mathrm{DHC}$ responses to sinusoidal stimulation improves dramatically with increasing stimulus amplitude. In figs. 31-35, A and $B$ represent the most common type (87.5\%) of cell encountered in this study. These cells respond well throughout the stimulus period at 5 and $10 \mathrm{~Hz}$, but adapt at 50 and $100 \mathrm{~Hz}$. The cell shown in $\mathrm{C}$ was unique in that it responded so well at $200 \mathrm{~Hz}$ (fig. $36 \mathrm{C})$. Two other cells were able to keep up with 50 and $100 \mathrm{~Hz}$ well with regard to firing rate and phase locking, but this cell did so at $200 \mathrm{~Hz}$ as well.

\section{Vector strength analysis}

Figs. 36-40 show the phase vectors for all of the $\mathrm{DHC}$ responses during sinusoidal stimuli at 5, 10,50, 100 and $200 \mathrm{~Hz}$, at amplitudes of $70,150,300$ and $500 \mu \mathrm{m}$. Only $200 \mathrm{~Hz}$ does not have a $500 \mu$ stimulation amplitude. Vector strength increases with increasing amplitude, as is evident in the phase vector diagrams as well as the summary histograms in figs. 41-45. Vector strengths tend to increase with

increasing amplitude, although this effect is not as apparent at frequencies greater than $50 \mathrm{~Hz}$. 
A

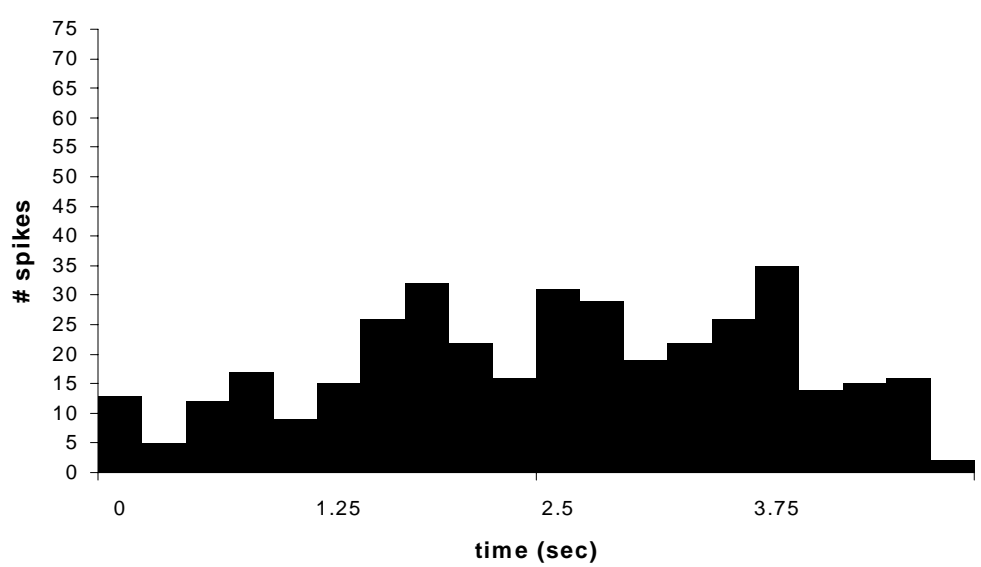

C
B

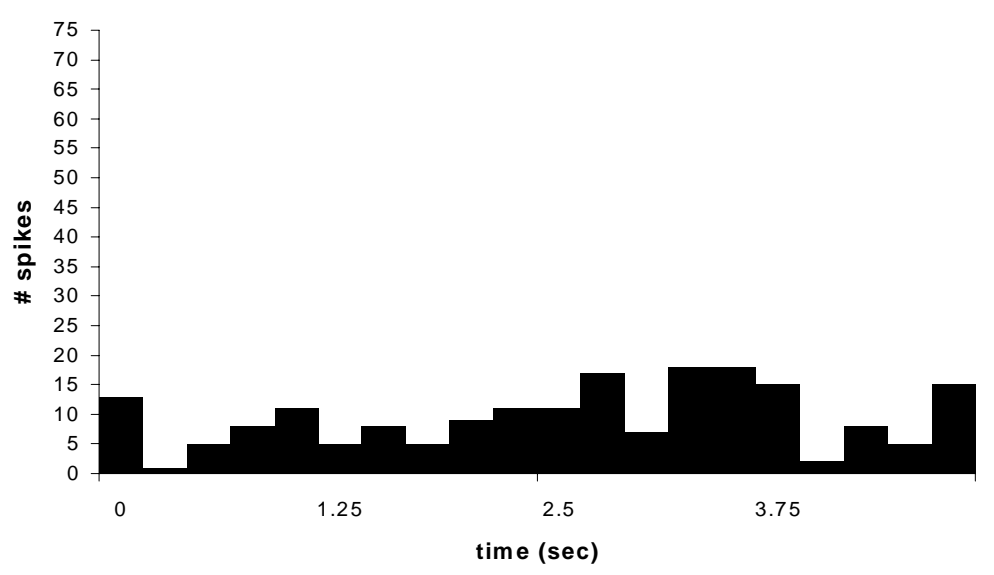

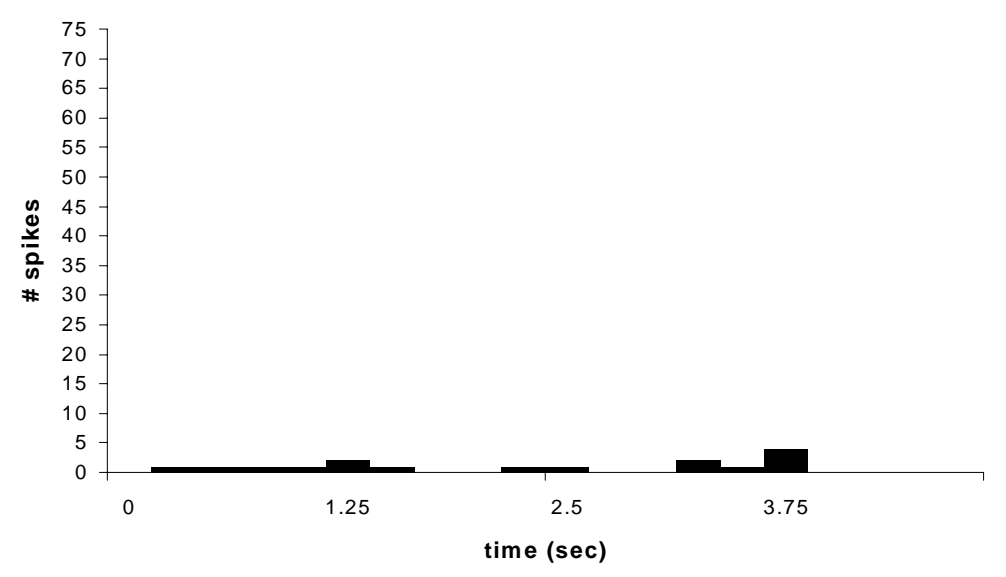

Fig 31. PSTHs of $3 \mathrm{DHC}$ responses during $5 \mathrm{~Hz}, 500 \mu$ sinusoidal stimulation. 
A

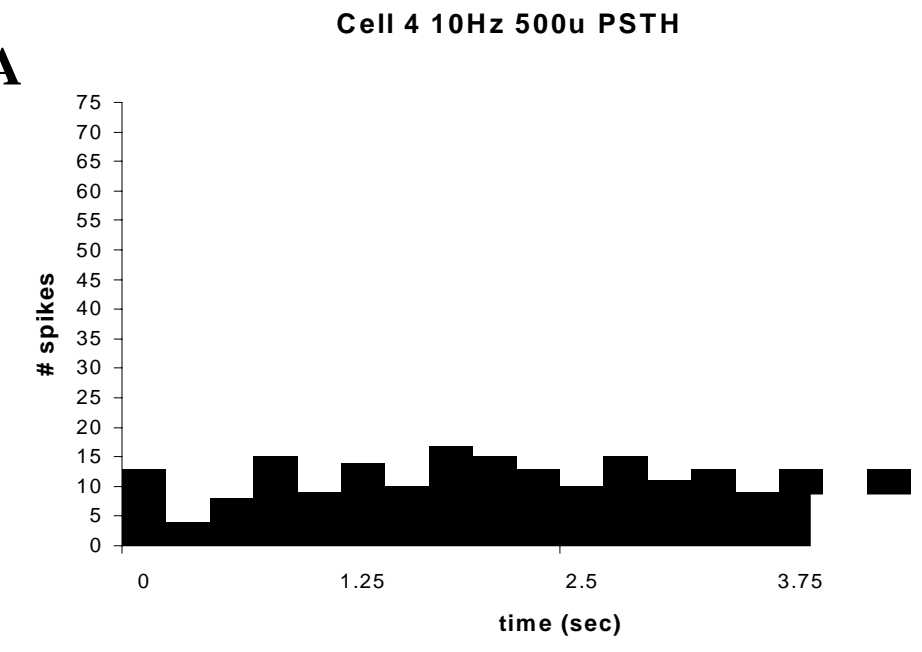

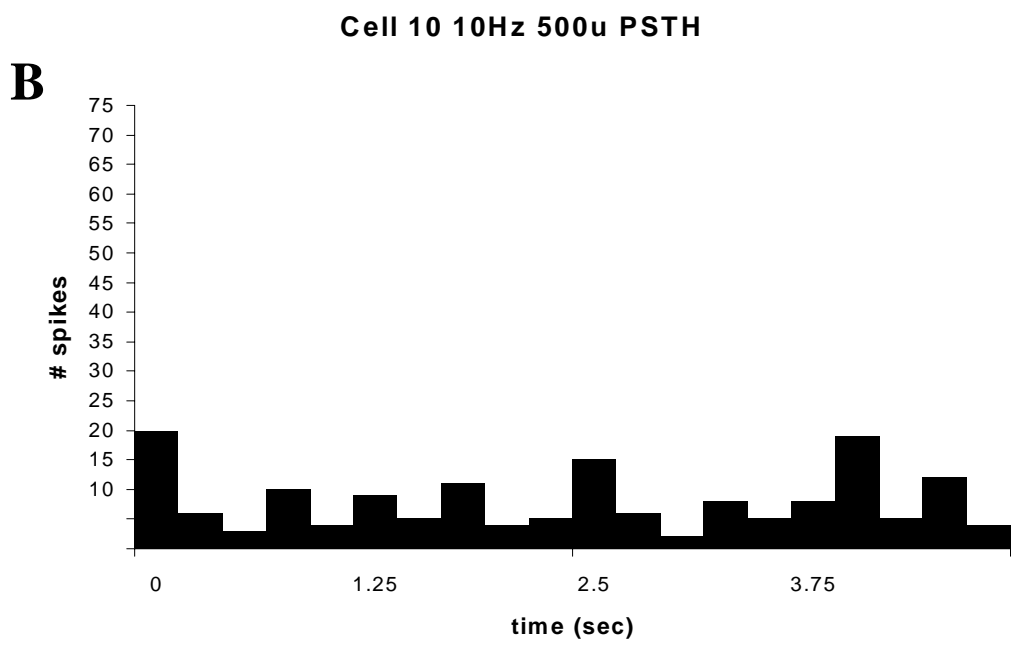

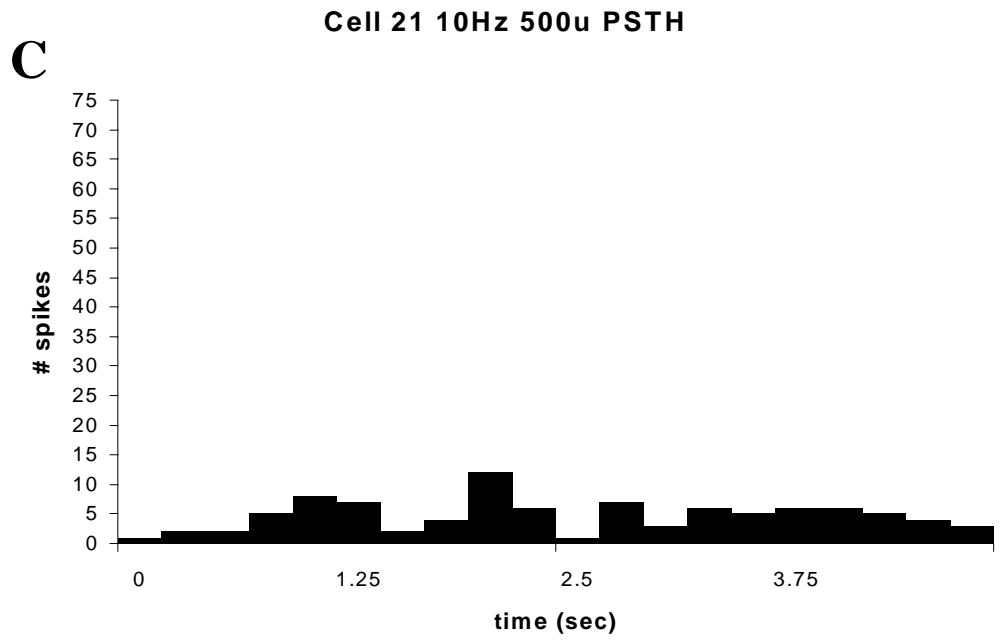

Fig. 32. PSTHs of $3 \mathrm{DHC}$ responses during $10 \mathrm{~Hz}, 500 \mu$ sinusoidal stimulation. 
A

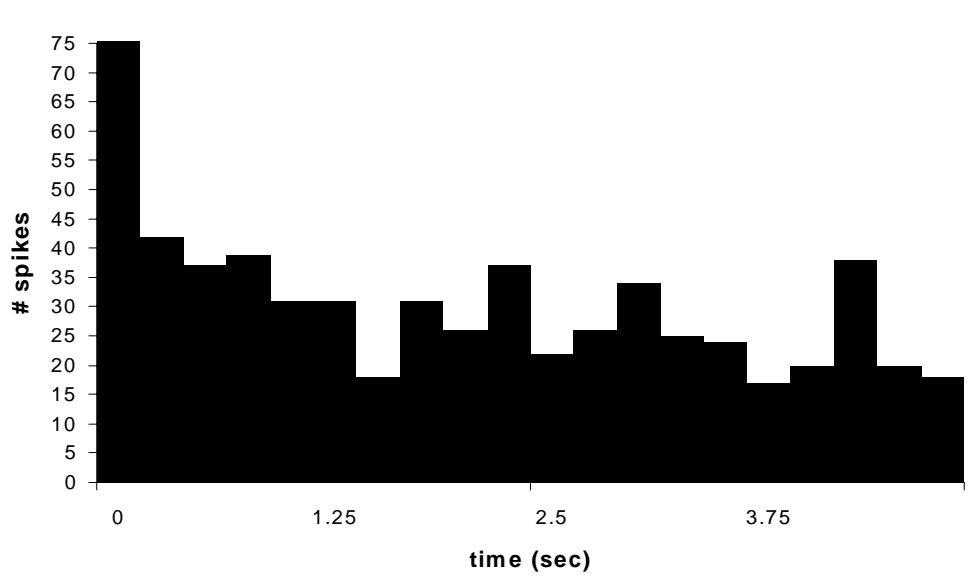

B

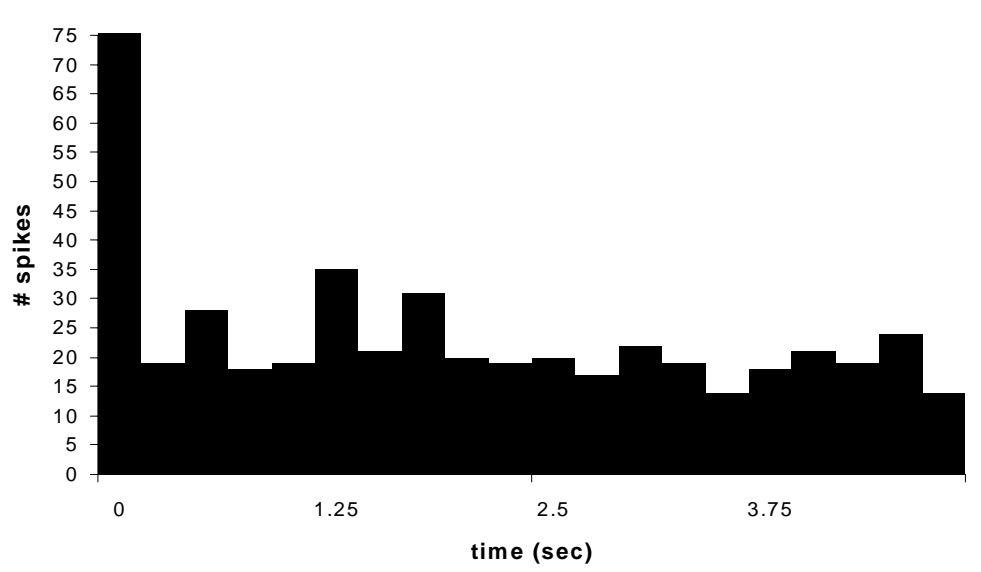

C

Cell $2150 \mathrm{~Hz} 500 \mathrm{u}$ PSTH

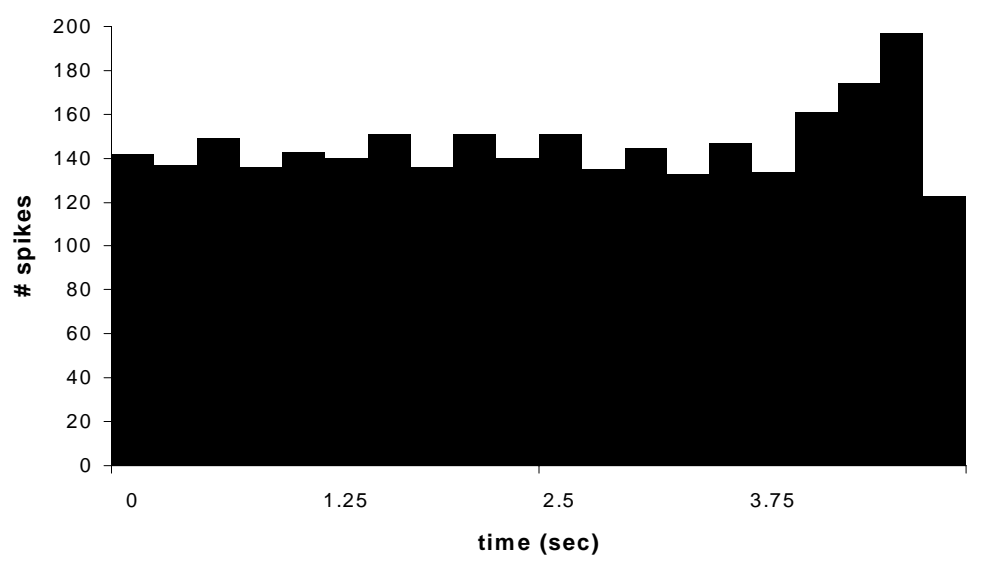

Fig. 33. PSTHs of $3 \mathrm{DHC}$ responses during $50 \mathrm{~Hz}, 500 \mu$ sinusoidal stimulation. 
A

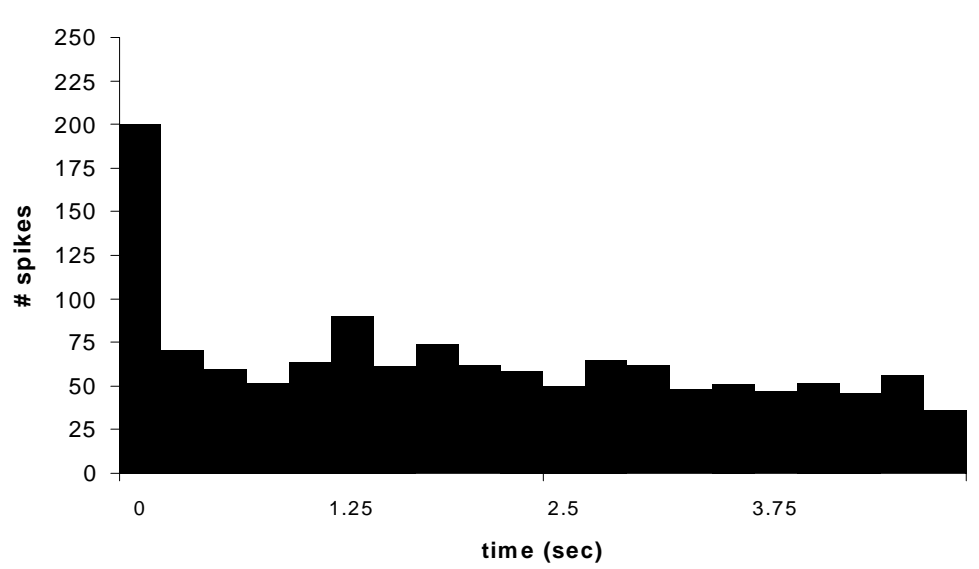

B

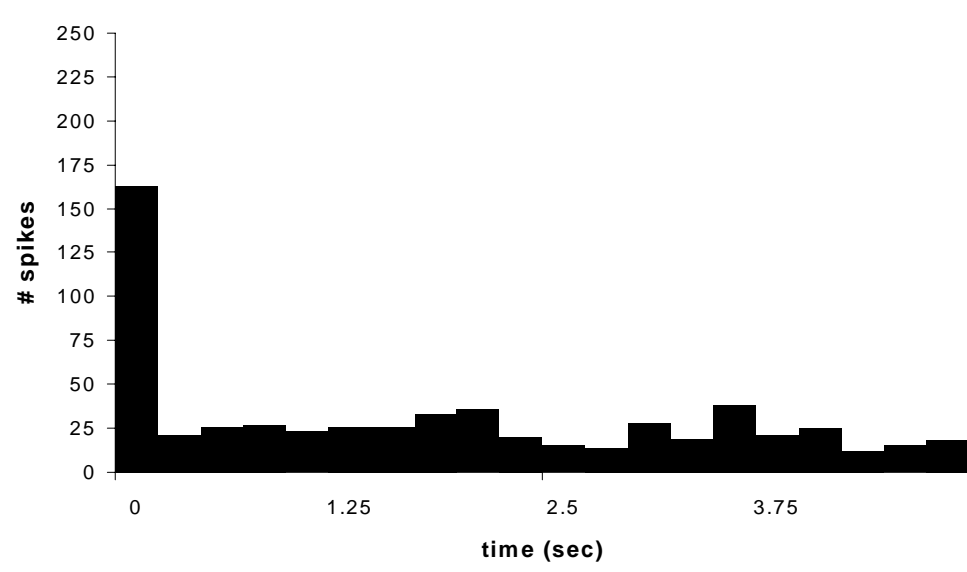

C

Cell $21100 \mathrm{~Hz} 500$ u PSTH

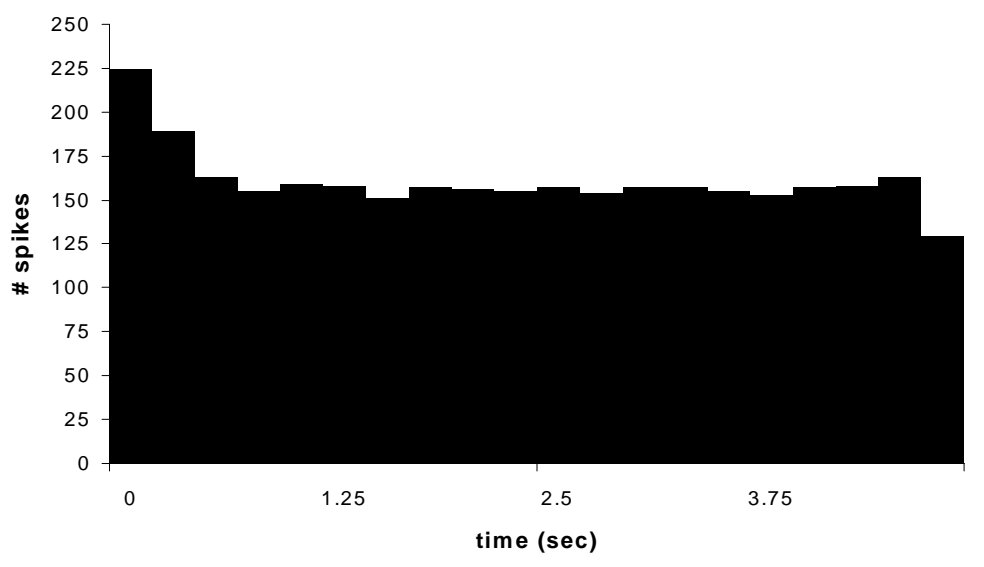

Fig. 34. PSTHs of $3 \mathrm{DHC}$ responses during $100 \mathrm{~Hz}, 500 \mu$ siusoidal stimulation. 
A Cell $4200 \mathrm{~Hz} 300 \mathrm{u}$ PSTH

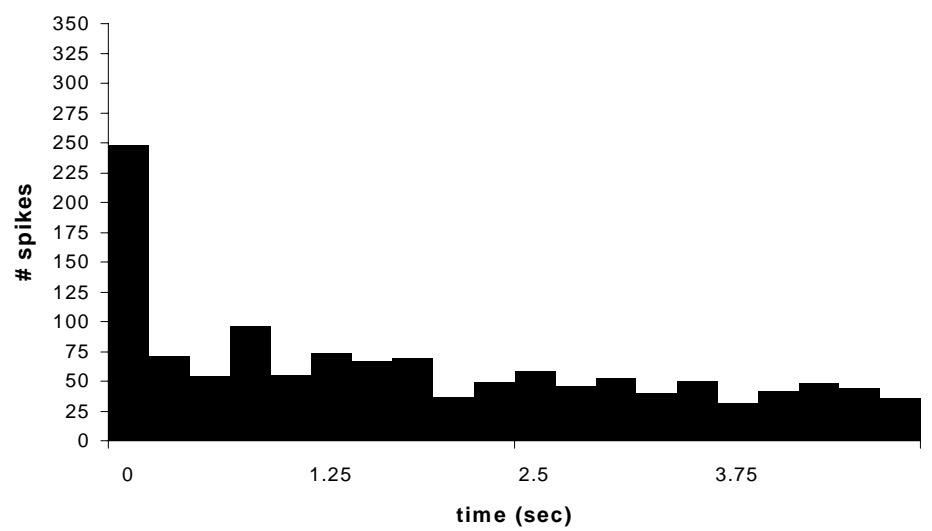

C Cell 21 200Hz 500u PSTH

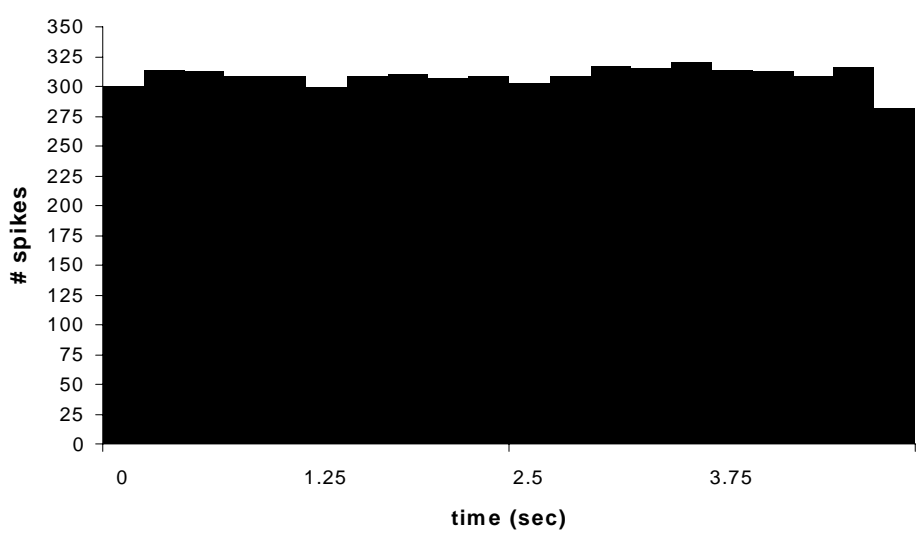

Fig. 35. PSTHs of $3 \mathrm{DHC}$ reponses during $200 \mathrm{~Hz} 300 \mu \mathrm{m}$ sinusoidal stimulation.
B Cell $10200 \mathrm{~Hz} 300 \mathrm{u}$ PSTH

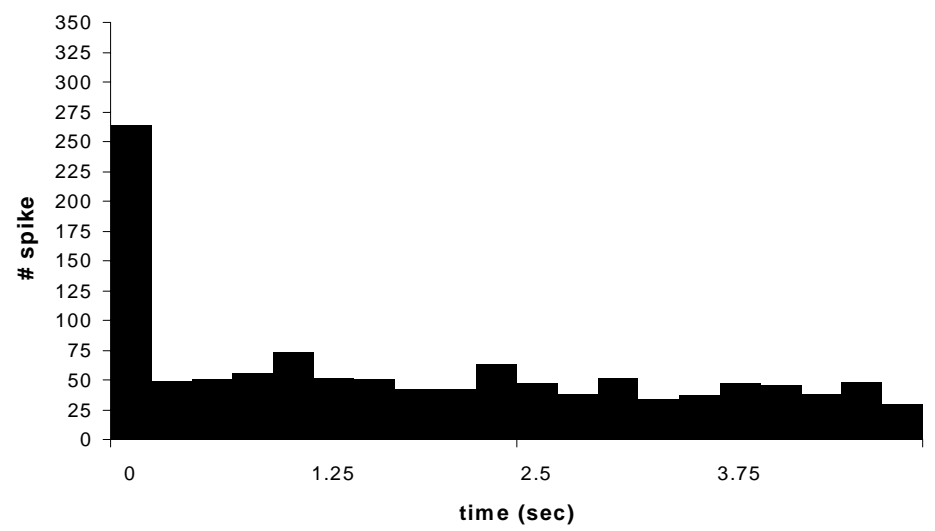


$70 \mu$

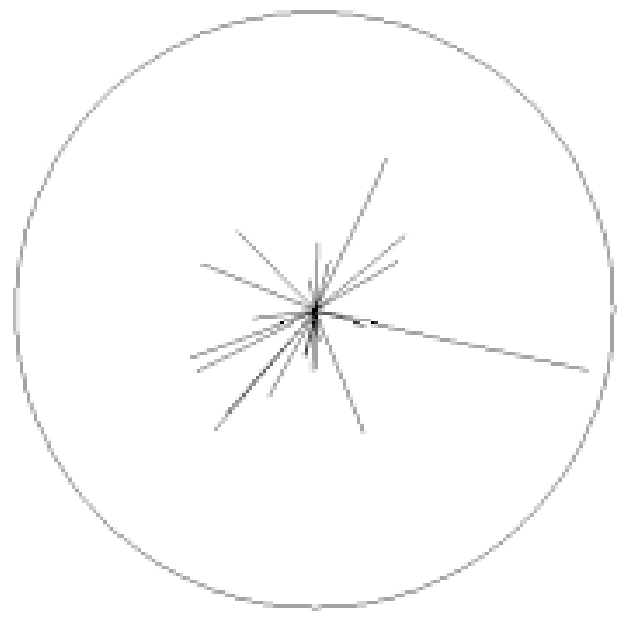

$300 \mu$

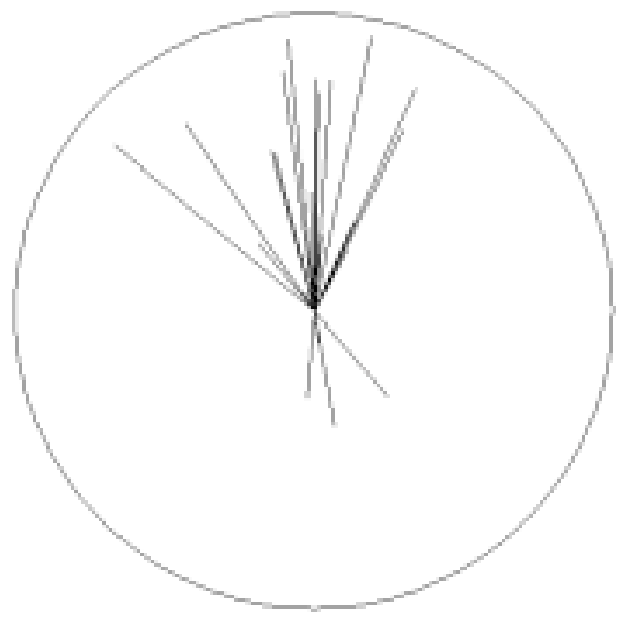

$150 \mu$

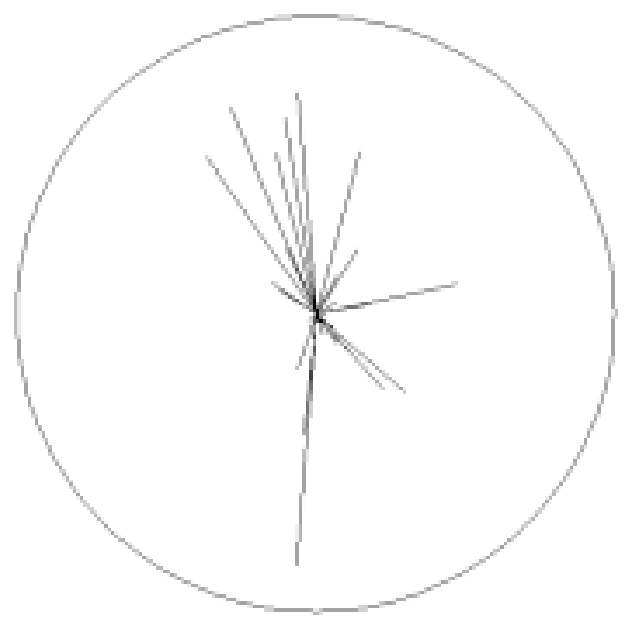

$500 \mu$

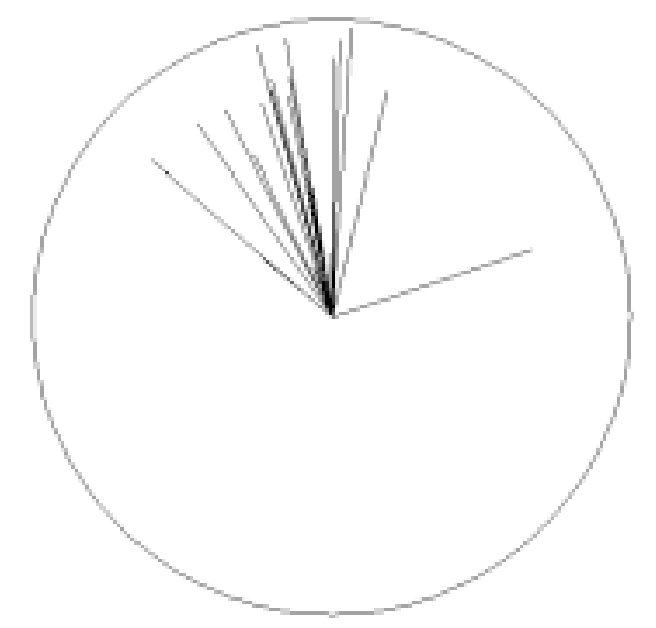

Fig. 36. Phase vectors of $\mathrm{DHC}$ responses to 4 amplitudes of $5 \mathrm{~Hz}$ sinusoidal stimulation. 

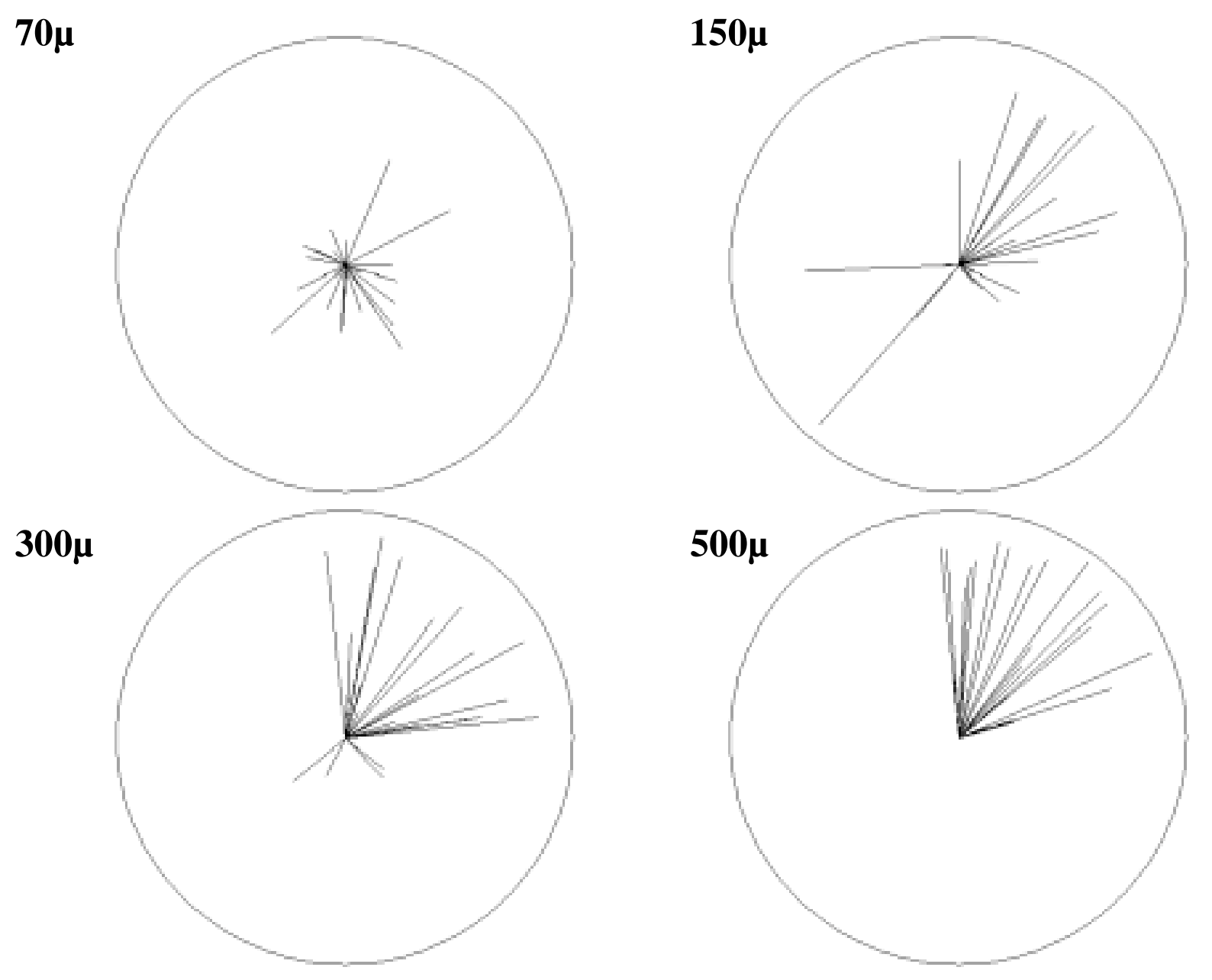

Fig. 37. Phase vectors of $\mathrm{DHC}$ responses to 4 amplitudes of $10 \mathrm{~Hz}$ sinusoidal stimulation. 

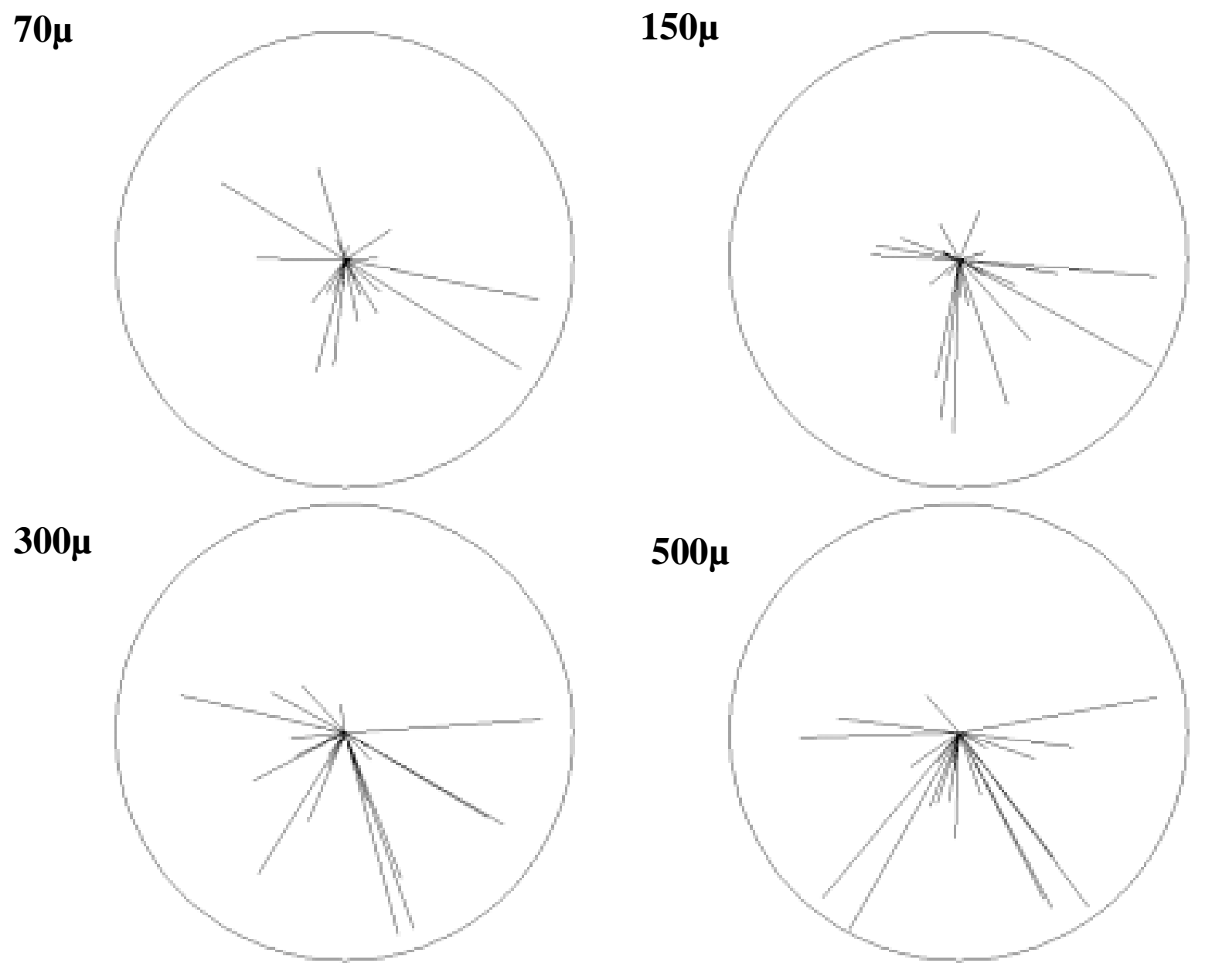

Fig. 38. Phase vectors of DHC responses to 4 amplitudes of $50 \mathrm{~Hz}$ sinusoidal stimulation. 
$70 \mu$

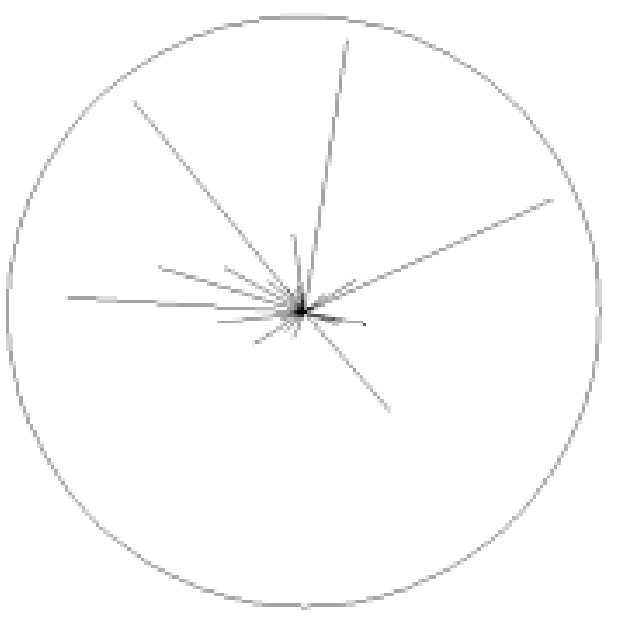

$300 \mu$

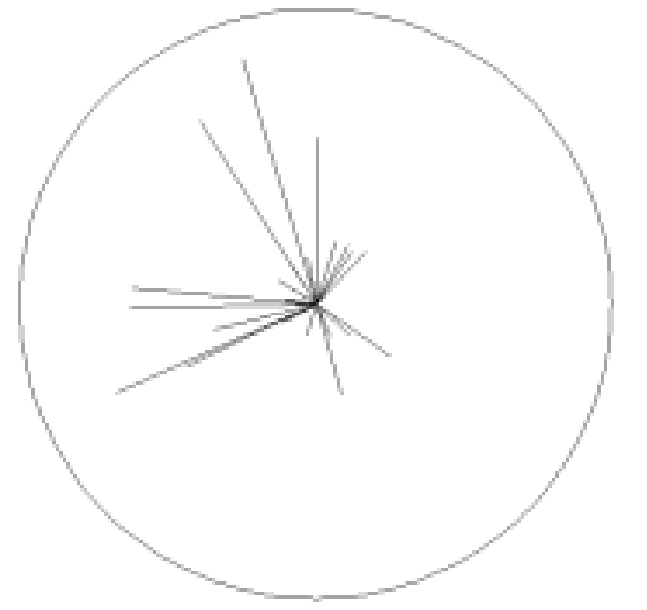

$150 \mu$

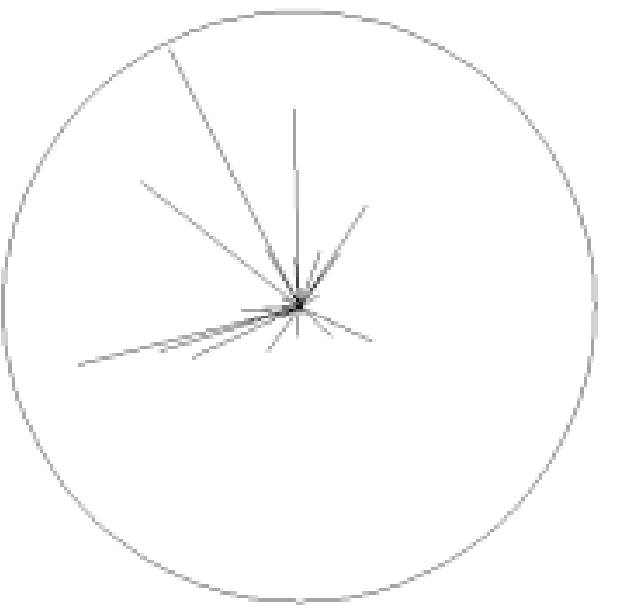

$500 \mu$

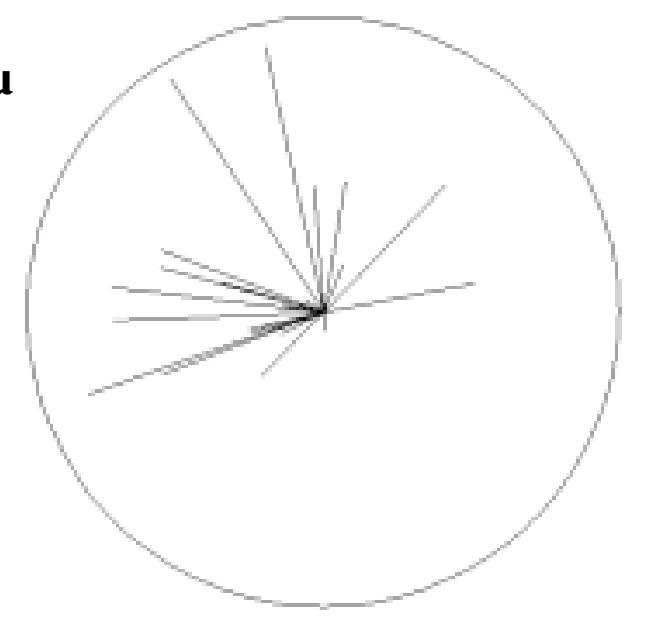

Fig. 39. Phase vectors of DHC responses to 4 amplitudes of $100 \mathrm{~Hz}$ sinusoidal stimulation. 
70u

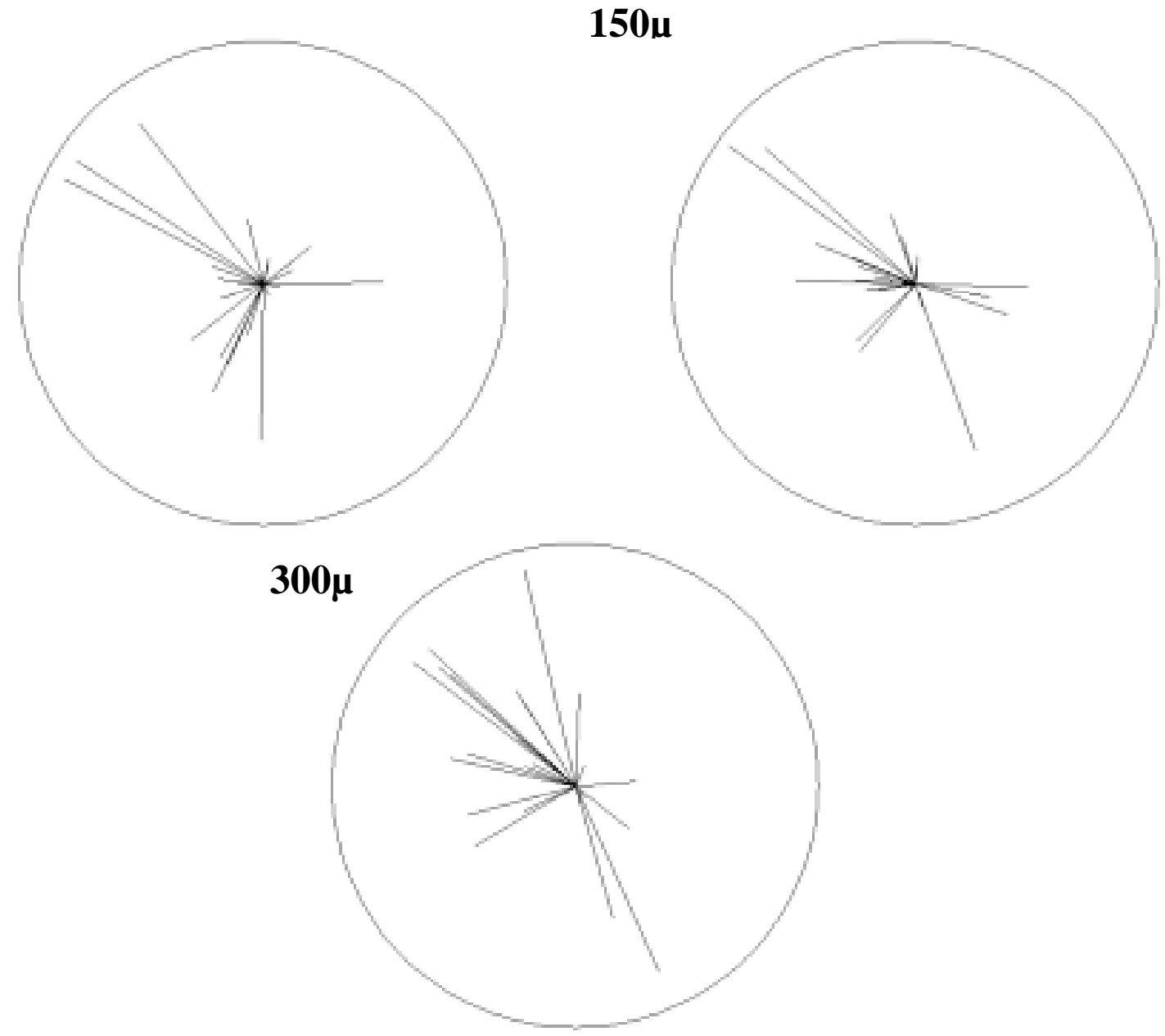

Fig. 40. Phase vectors of $\mathrm{DHC}$ responses to 3 amplitudes of $200 \mathrm{~Hz}$ sinusoidal stimulation. 
A

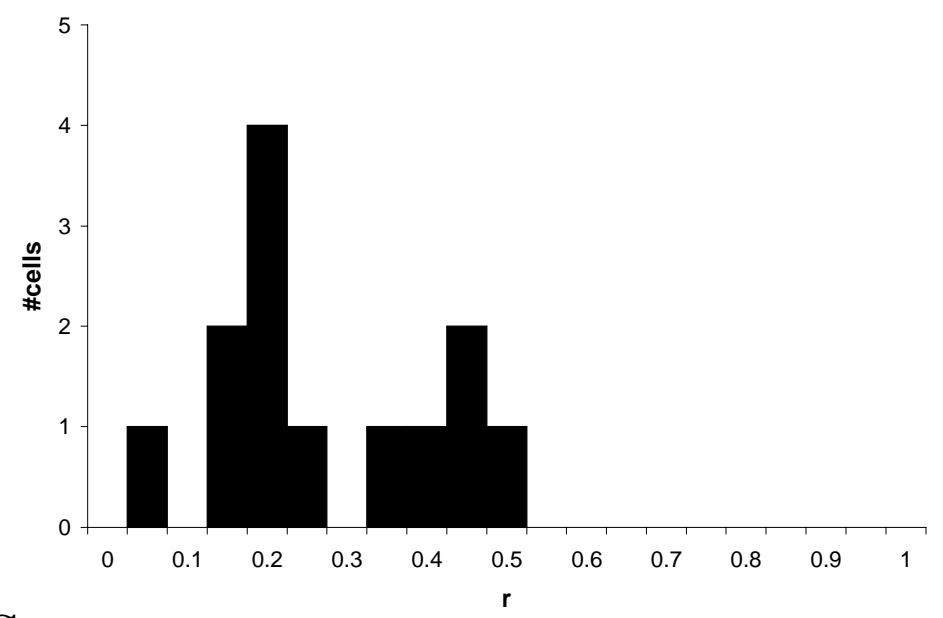

C

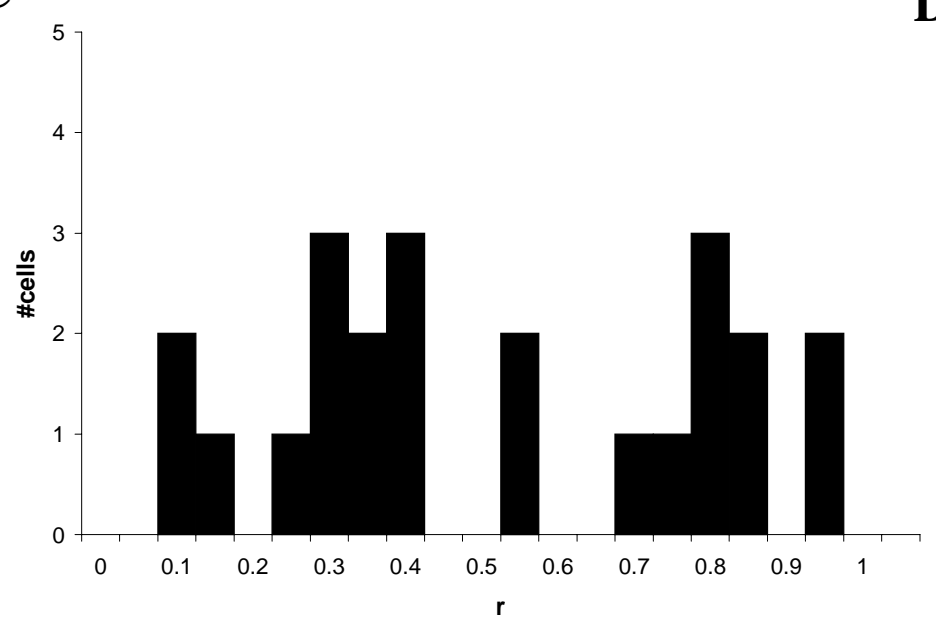

B

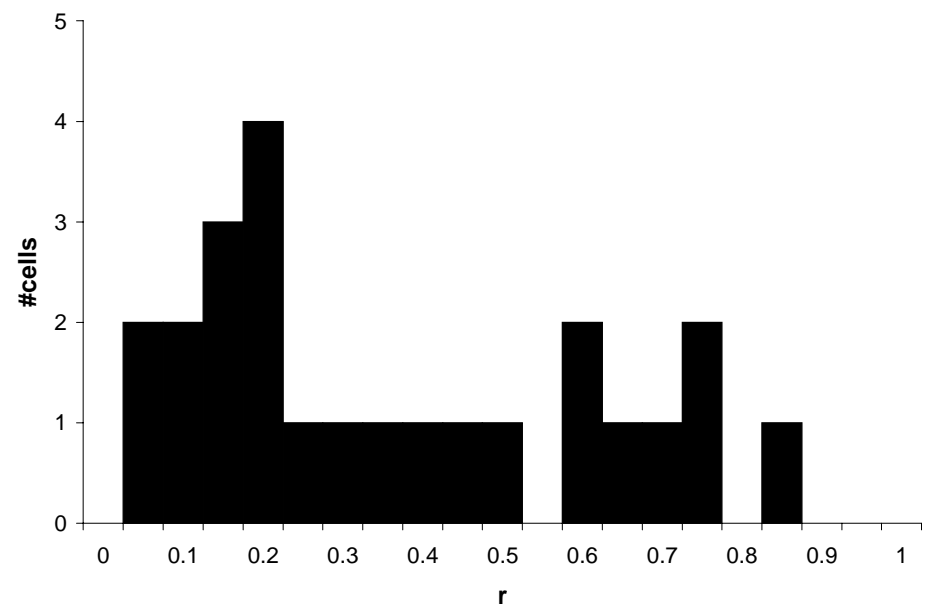

D

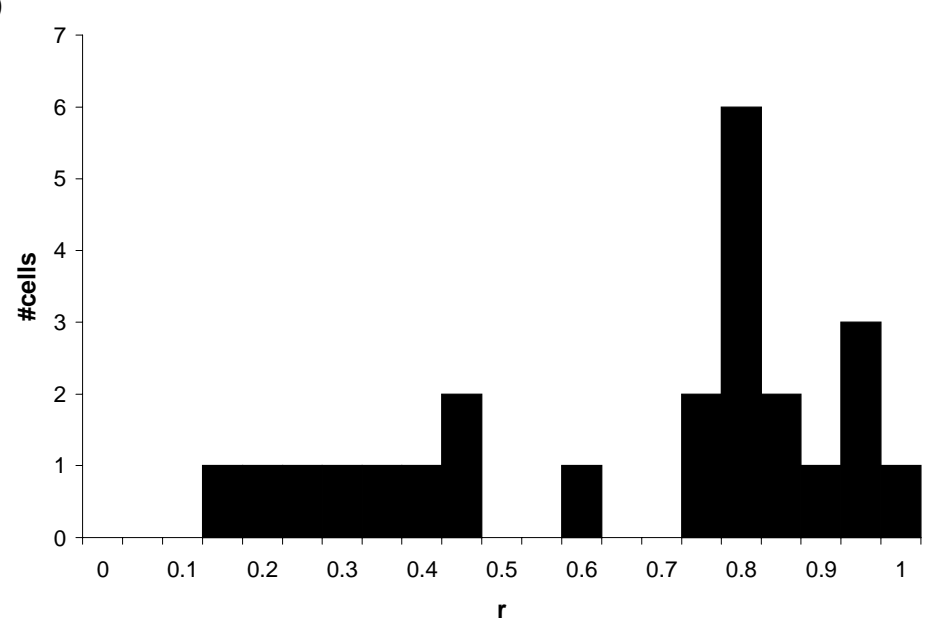

Fig. 41. Summary histograms of vector strengths of $\mathrm{DHC}$ responses to $5 \mathrm{~Hz}$ sinusoidal stimulation at 4 amplitudes: A-D, 70, 150, 300 and $500 \mu \mathrm{m}$. 
A

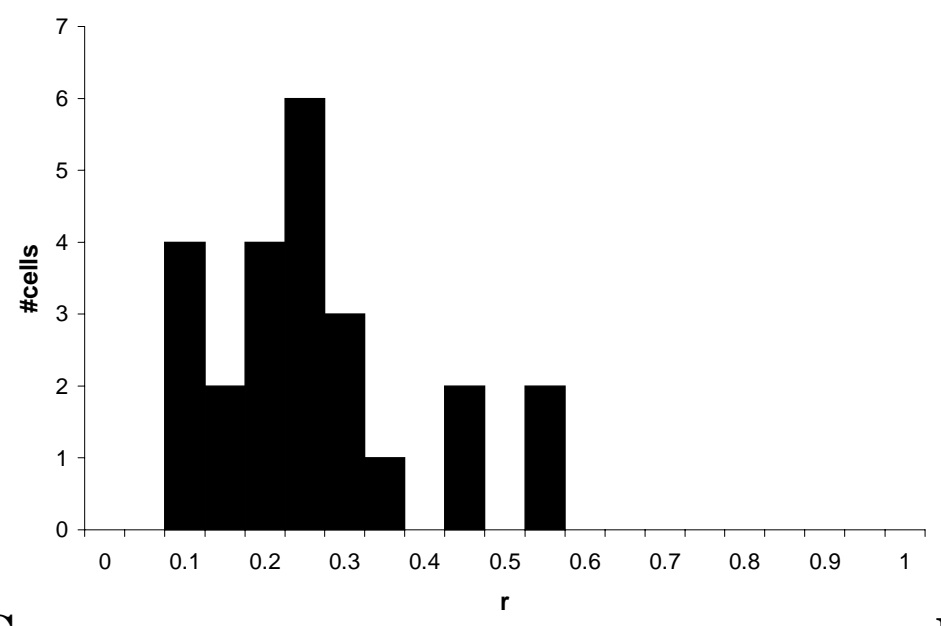

C

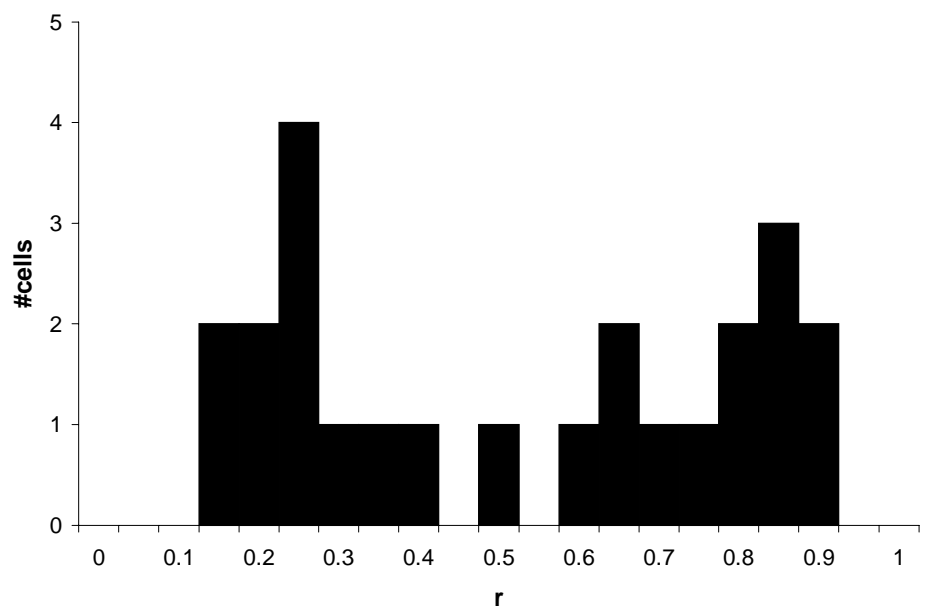

B

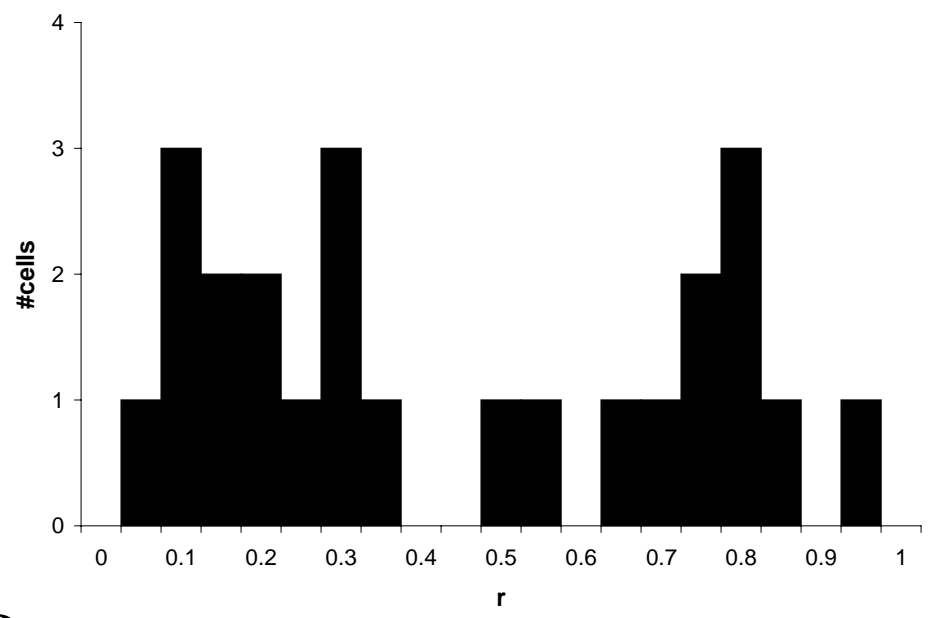

D

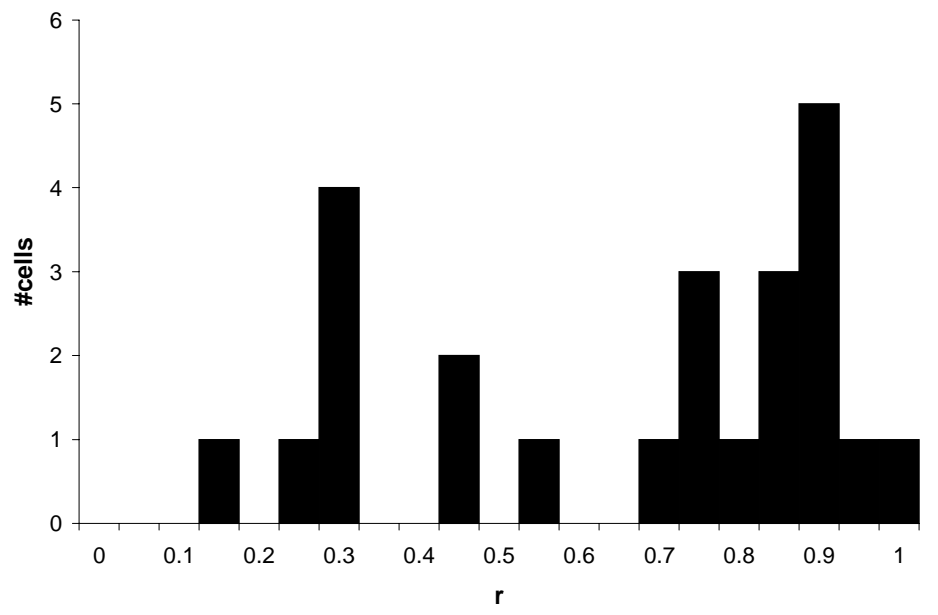

Fig. 42. Summary histograms of vector strengths of $\mathrm{DHC}$ responses to $10 \mathrm{~Hz}$ sinusoidal stimulation at 4 amplitudes: A-D, 70, 150, 300 and 500 $\mu$ m. 
A

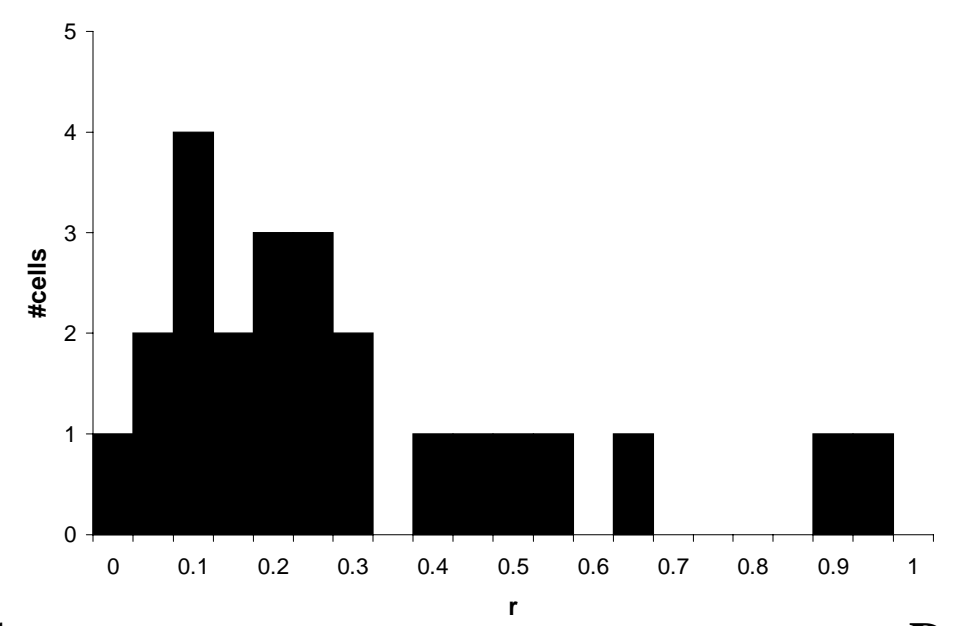

C

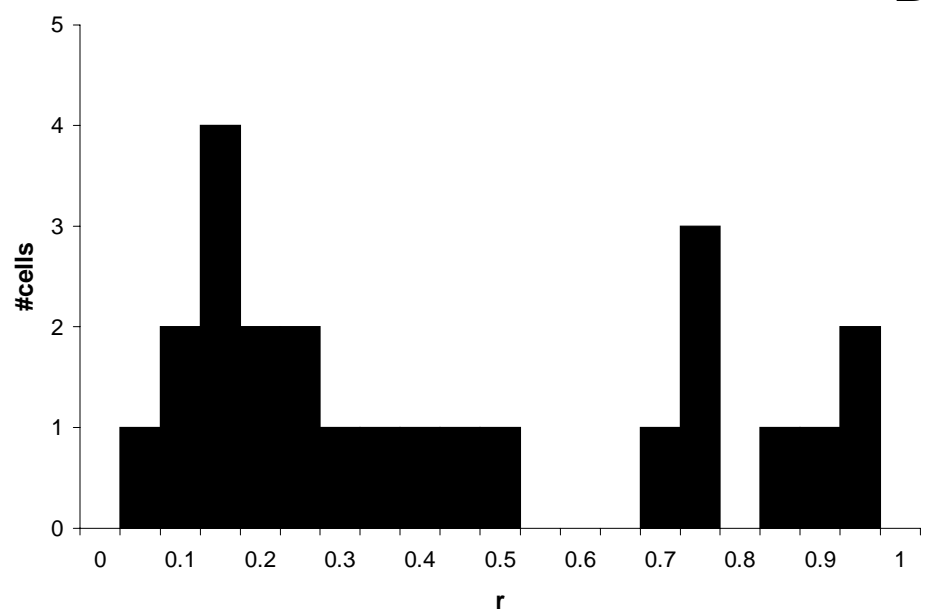

B

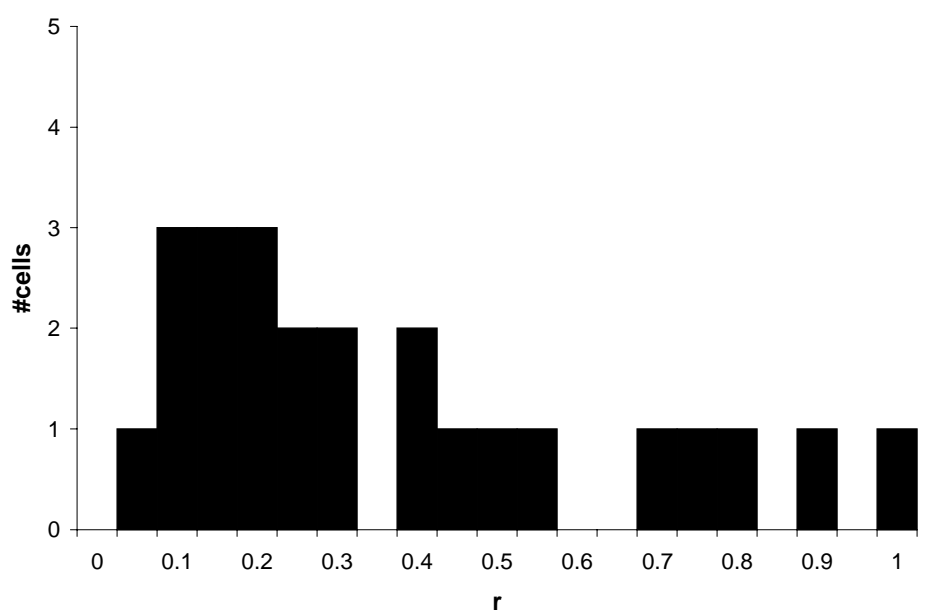

D

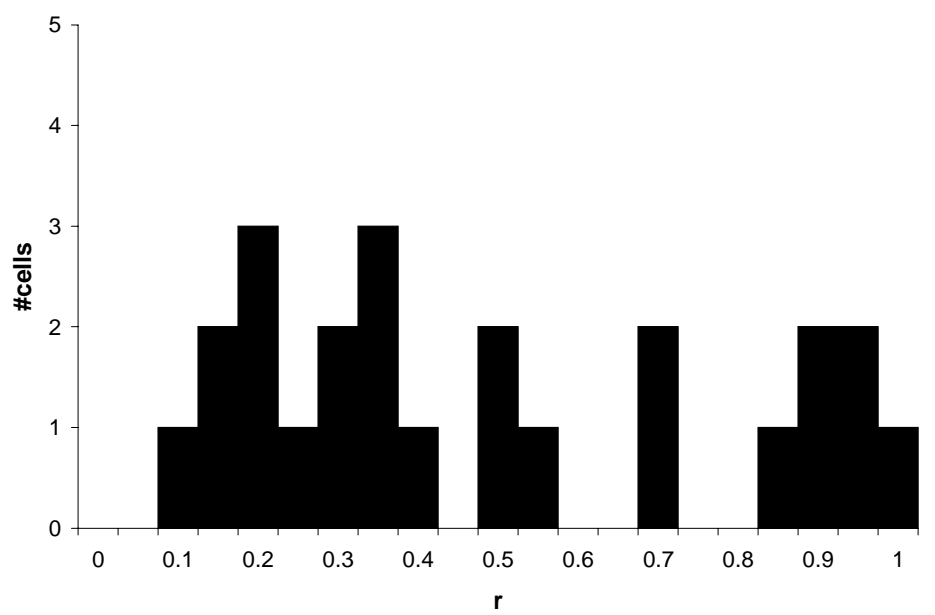

Fig. 43. Summary histograms of vector strengths of $\mathrm{DHC}$ responses to $50 \mathrm{~Hz}$ sinusoidal stimulation at 4 amplitudes: A-D, 70, 150, 300 and 500 $\mu \mathrm{m}$. 
A

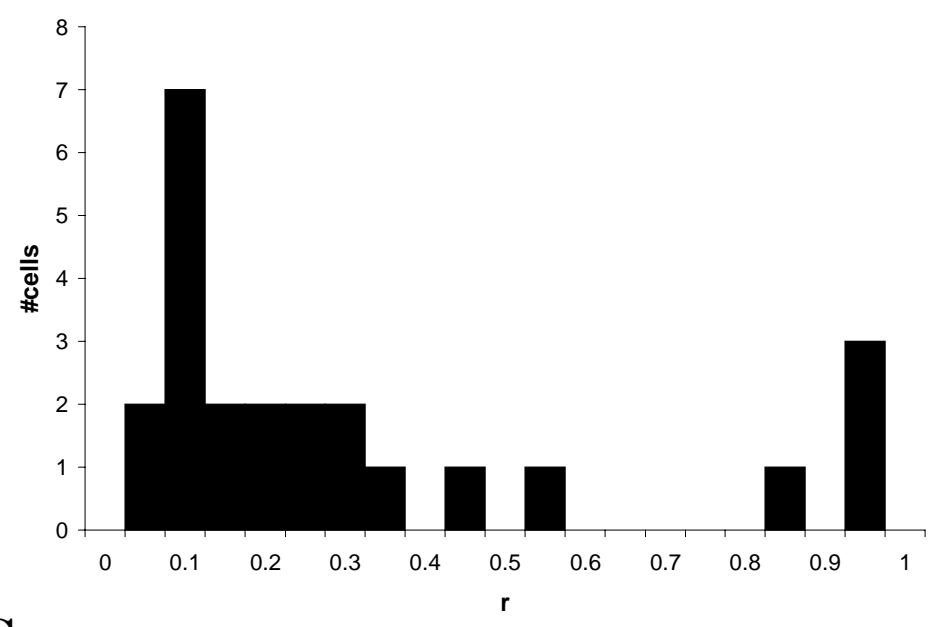

C

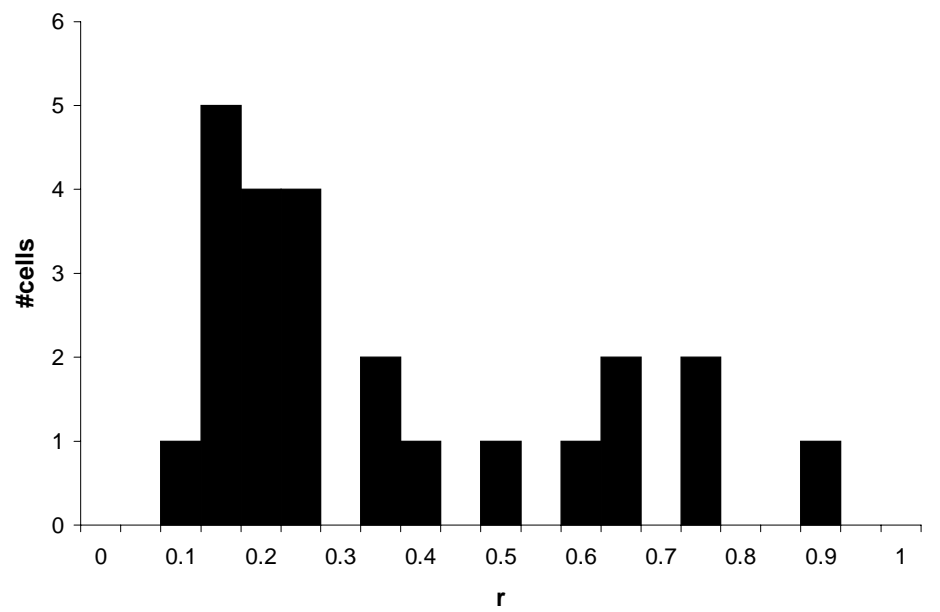

B

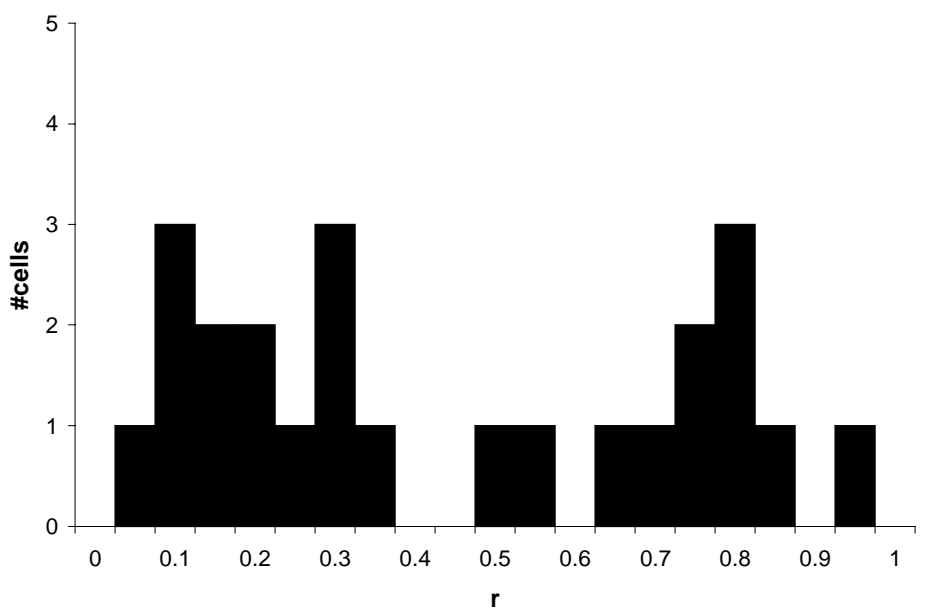

D

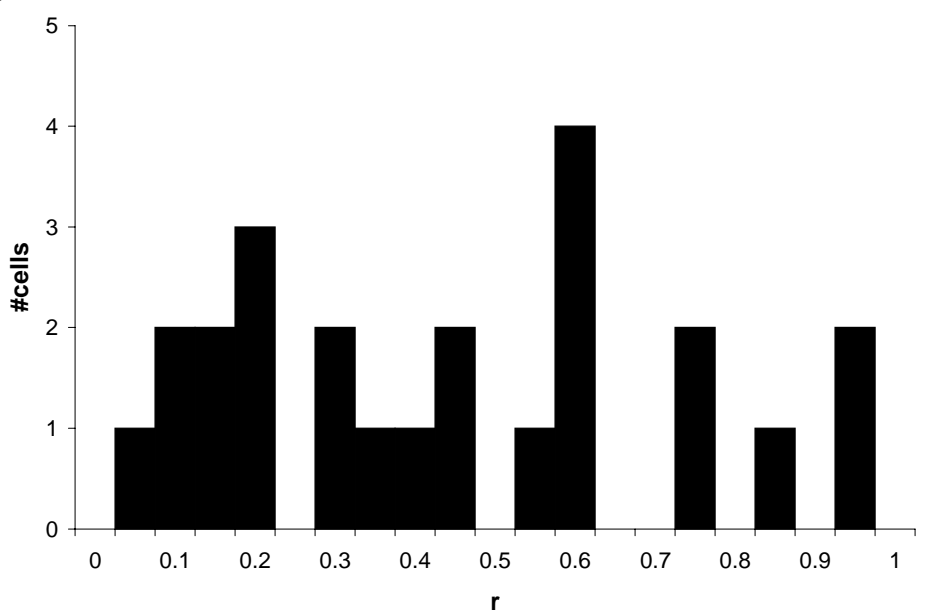

Fig. 44. Summary histograms of vector strengths of $\mathrm{DHC}$ responses to $100 \mathrm{~Hz}$ sinusoidal stimulation at 4 amplitudes: A-D, 70, 150, 300 and $500 \mu$. 
A

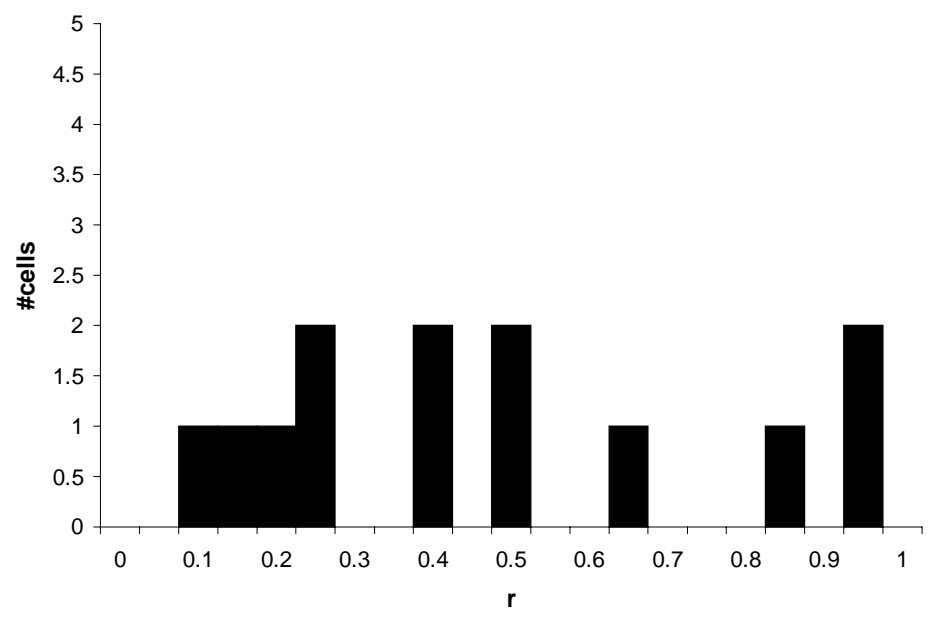

B

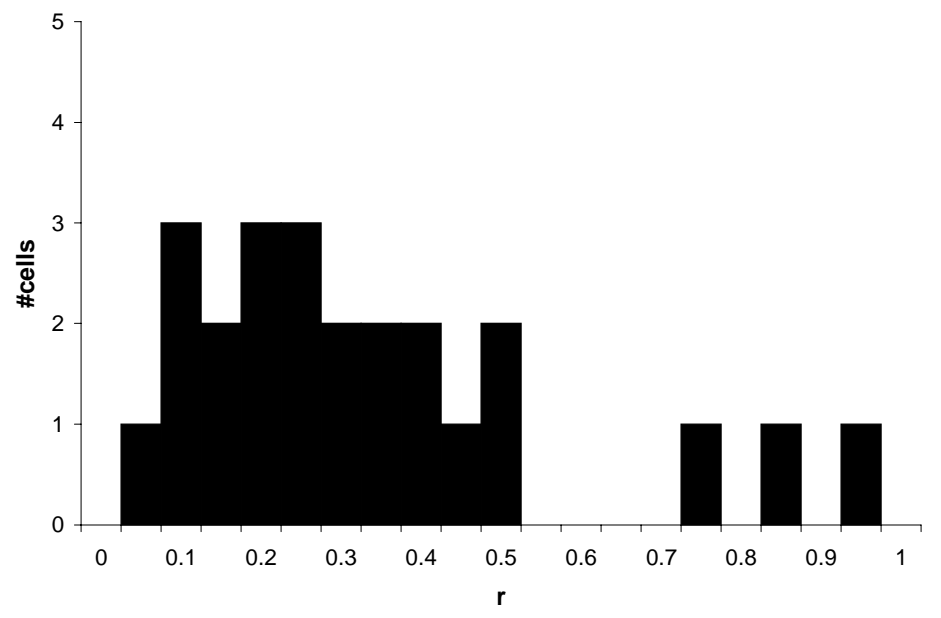

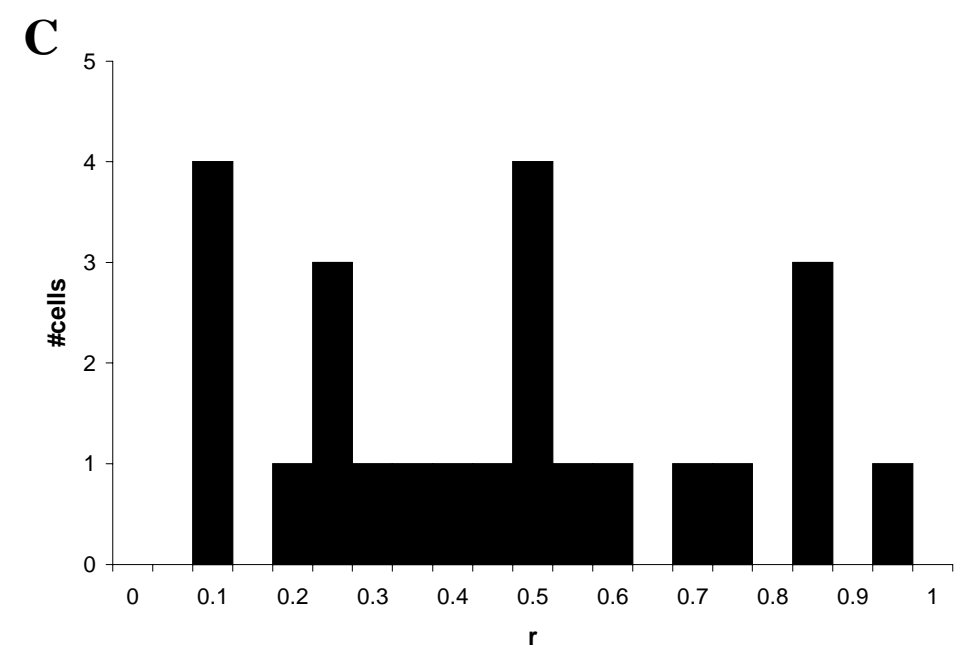

Fig. 45. Summary histograms of vector strengths of $\mathrm{DHC}$ responses to $200 \mathrm{~Hz}$ sinusoidal stimulation at 3 amplitudes: A-C, 70, 150 and $300 \mu \mathrm{m}$. 
The relationship between stimulus amplitude at a given frequency and vector strength is shown in fig. 46. This graph demonstrates that there is definitely a positive relationship between the two. Fig. 47 shows the relationship between frequency at a given amplitude and vector strength. This relationship is less clear, as vector strength goes up after $100 \mathrm{~Hz}$ for all amplitudes except $500 \mu$. In general, the vector strength goes down as stimulus frequency goes up.

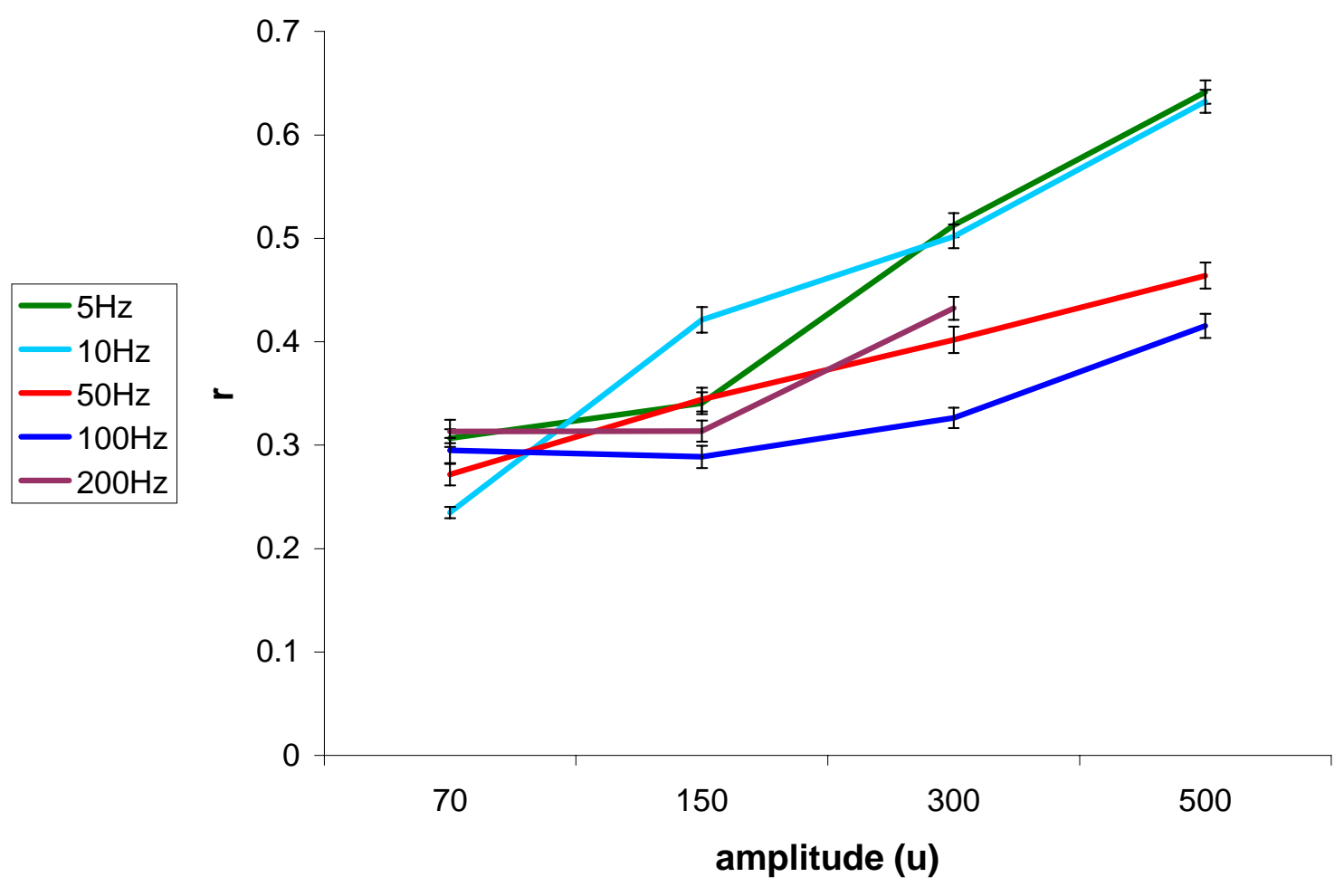

Fig. 46. Vector strength vs. amplitude at 5 frequencies of stimulation. 


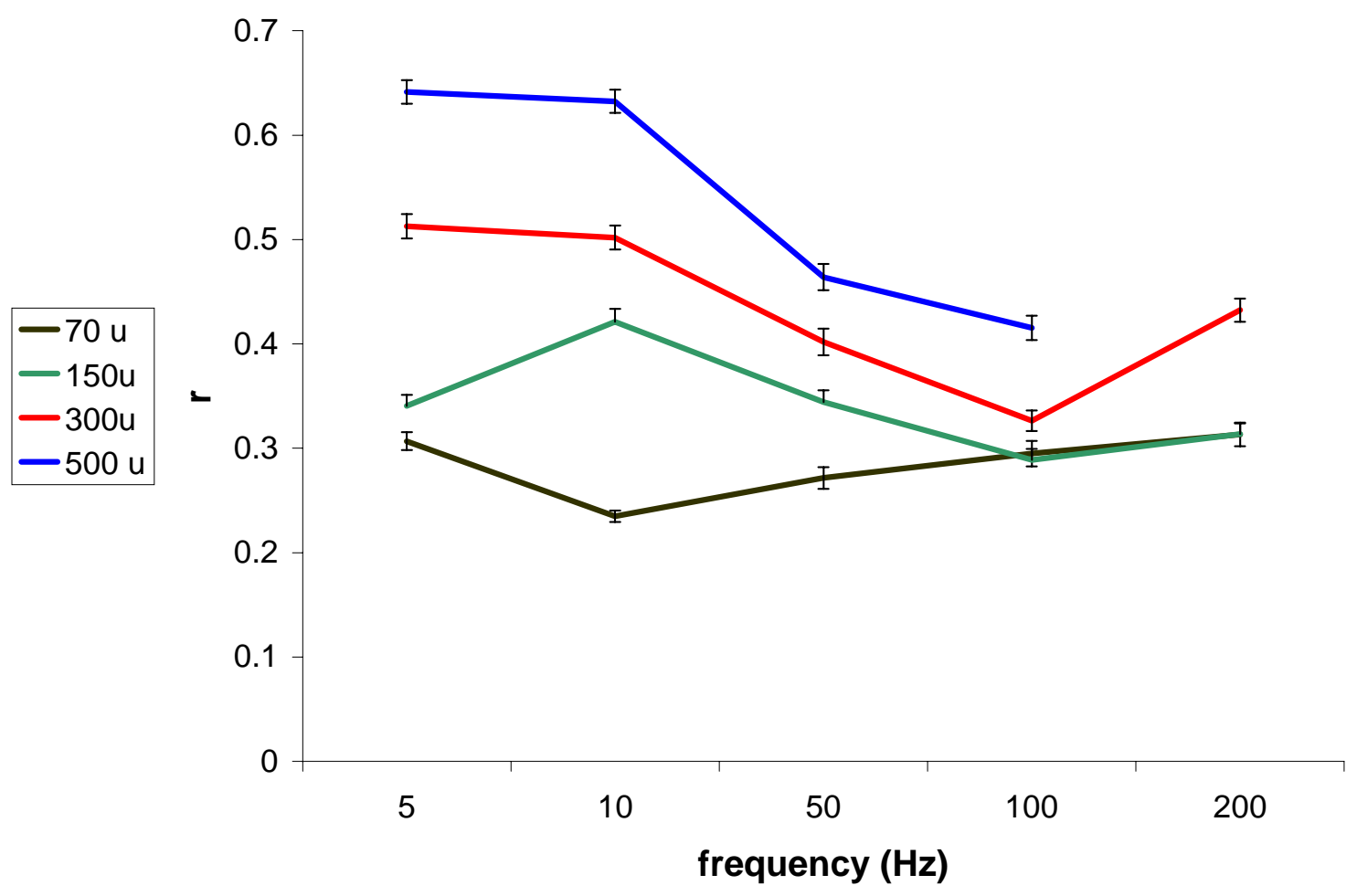

Fig. 47. Vector strength vs. frequency at 4 amplitudes of stimulation.

\section{Analysis of power spectra}

Principal component frequencies as determined by power spectrum analysis are shown in figs. 48-52. As one might expect from the vector strength results, the distribution of principal components is highly dependent on amplitude. As stimulus amplitude increases (from $A$ to $D$ in these figures), the principal frequency components of the responses tend to cluster at the stimulus frequency and its harmonics. This is true even at higher frequencies. 


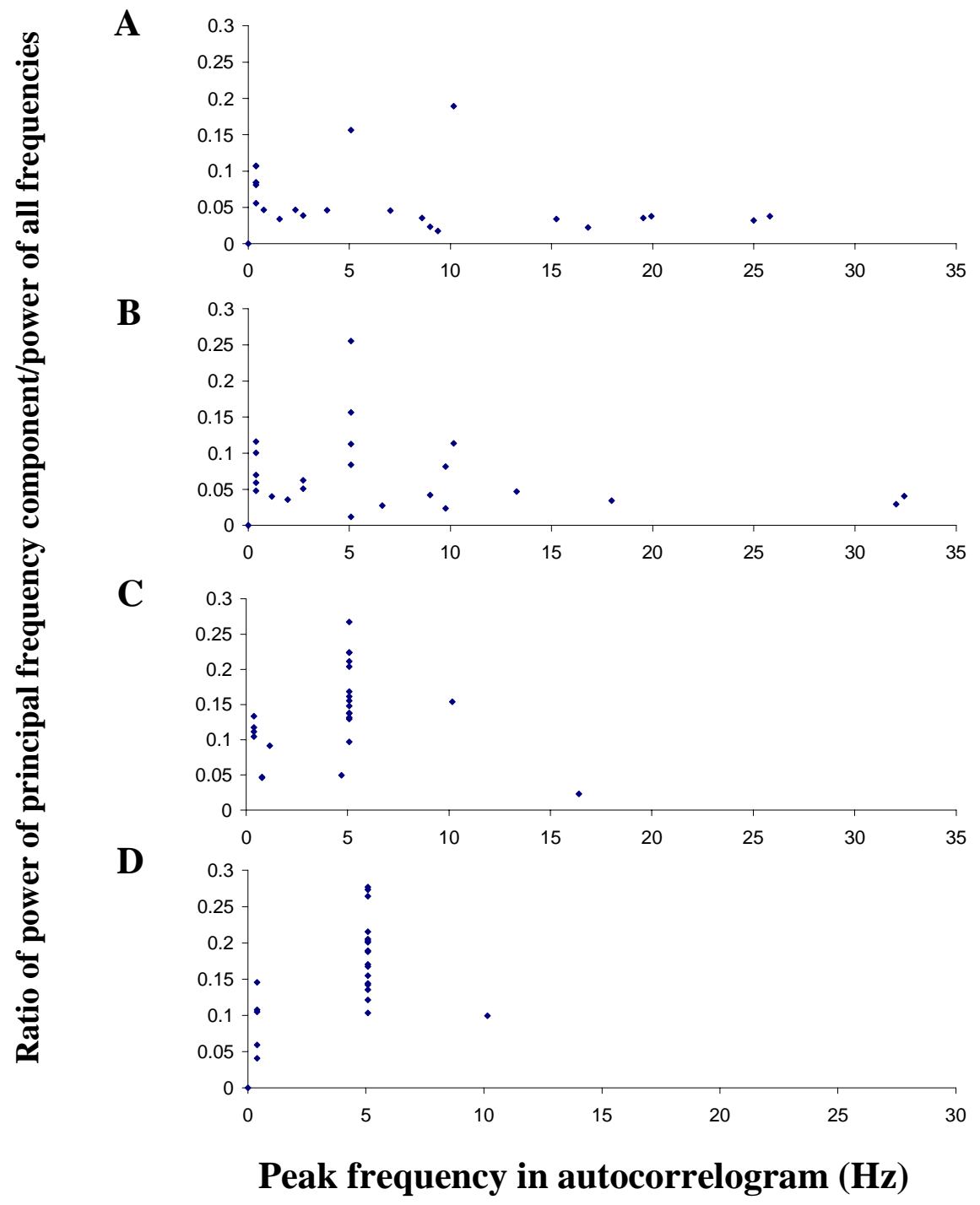

Fig. 48. Principal frequency components of $\mathrm{DHC}$ responses to $5 \mathrm{~Hz}$ sinusoidal

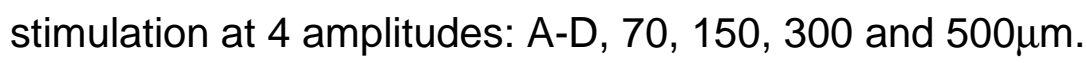




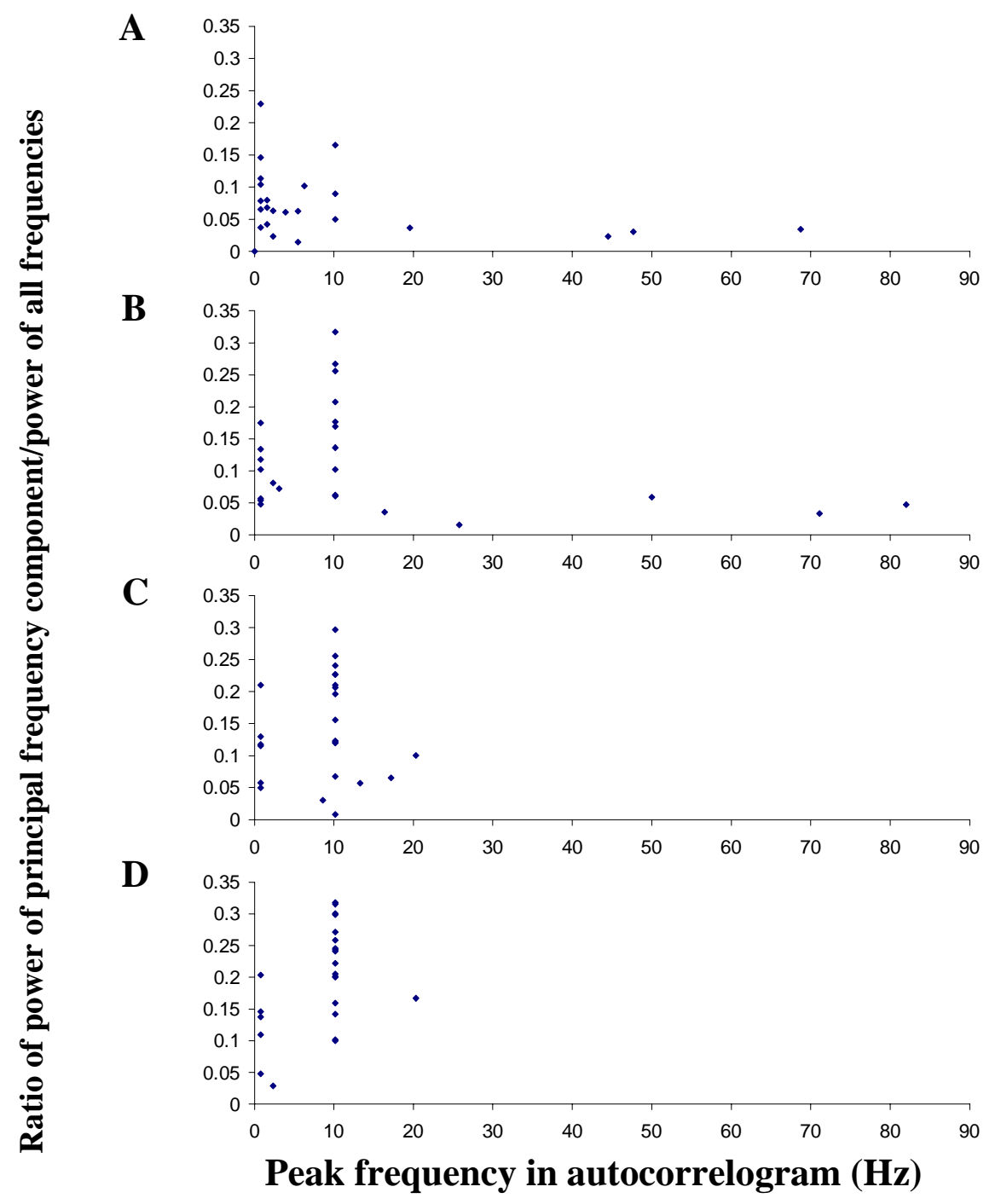

Fig. 49. Principal frequency components of $\mathrm{DHC}$ responses to $10 \mathrm{~Hz}$ sinusoidal stimulation at 4 amplitudes: A-D, 70, 105, 300, and 500 $\mu \mathrm{m}$. 


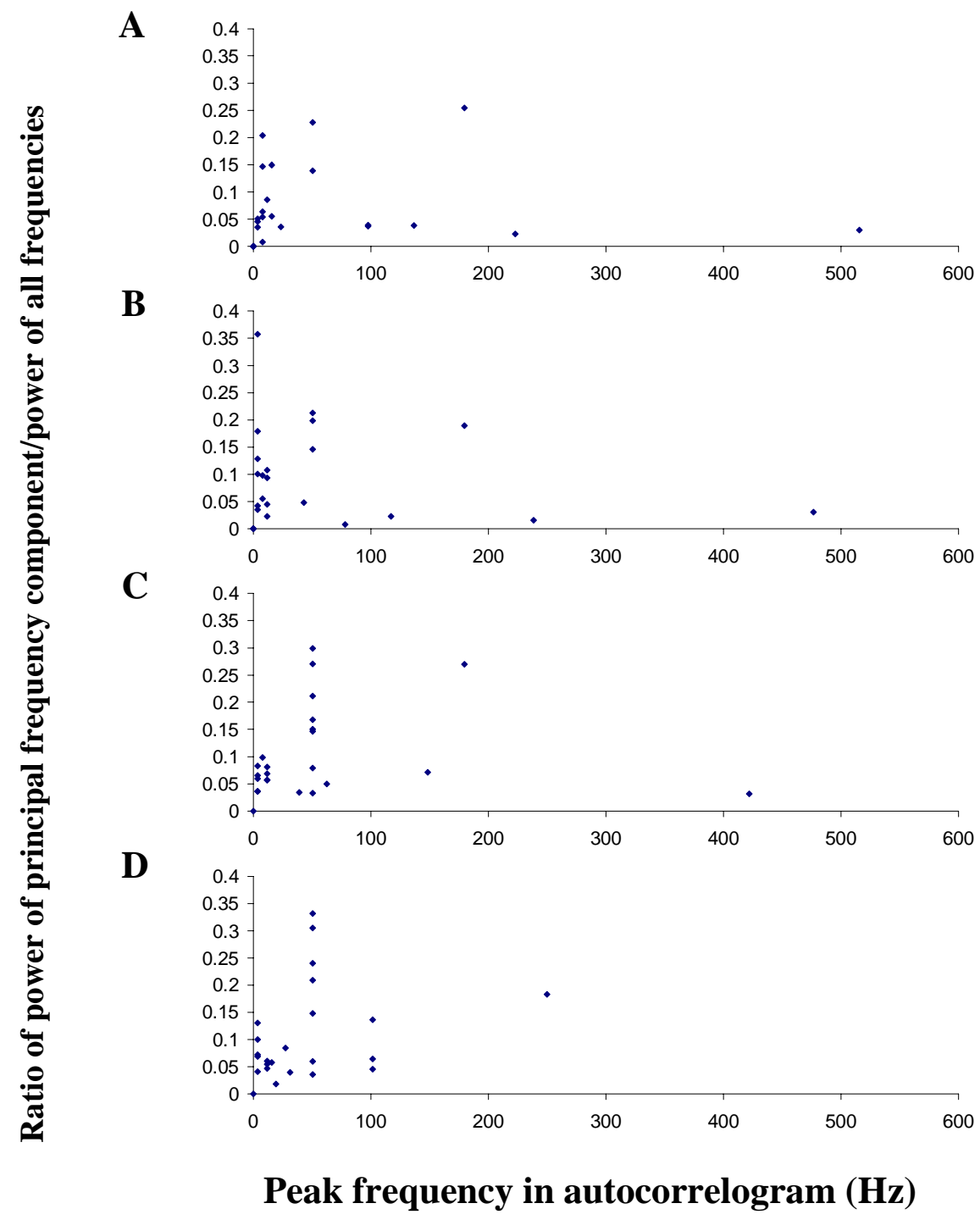

Fig. 50. Principal frequency components of $\mathrm{DHC}$ responses to $50 \mathrm{~Hz}$ sinusoidal

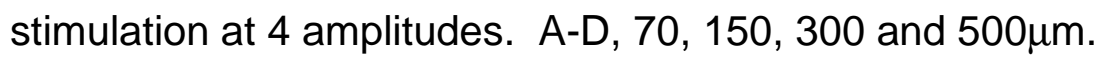




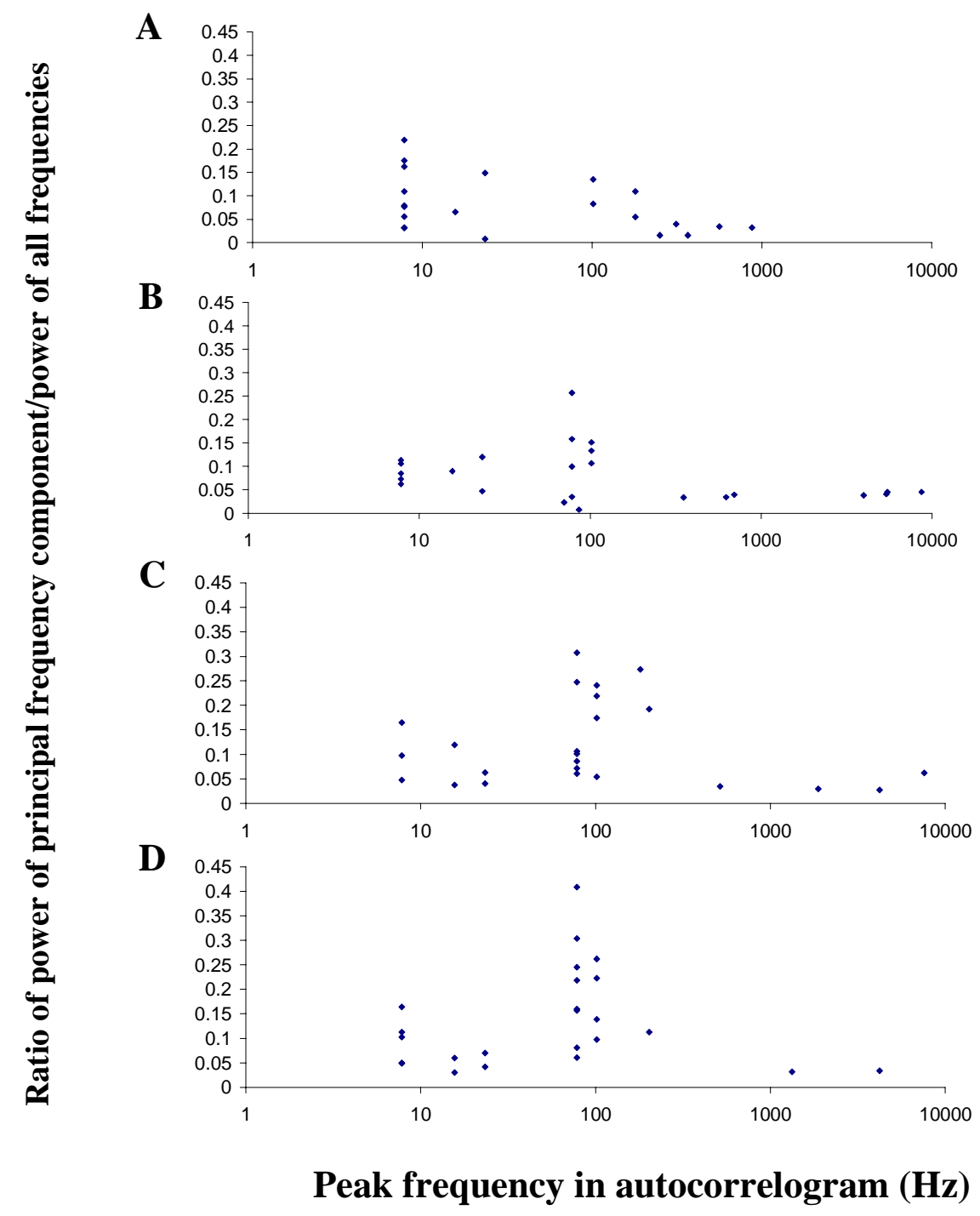

Fig. 51 Principal frequency components of DHC responses to $100 \mathrm{~Hz}$ sinusoidal

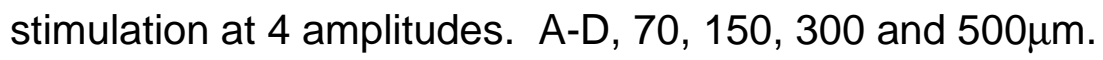




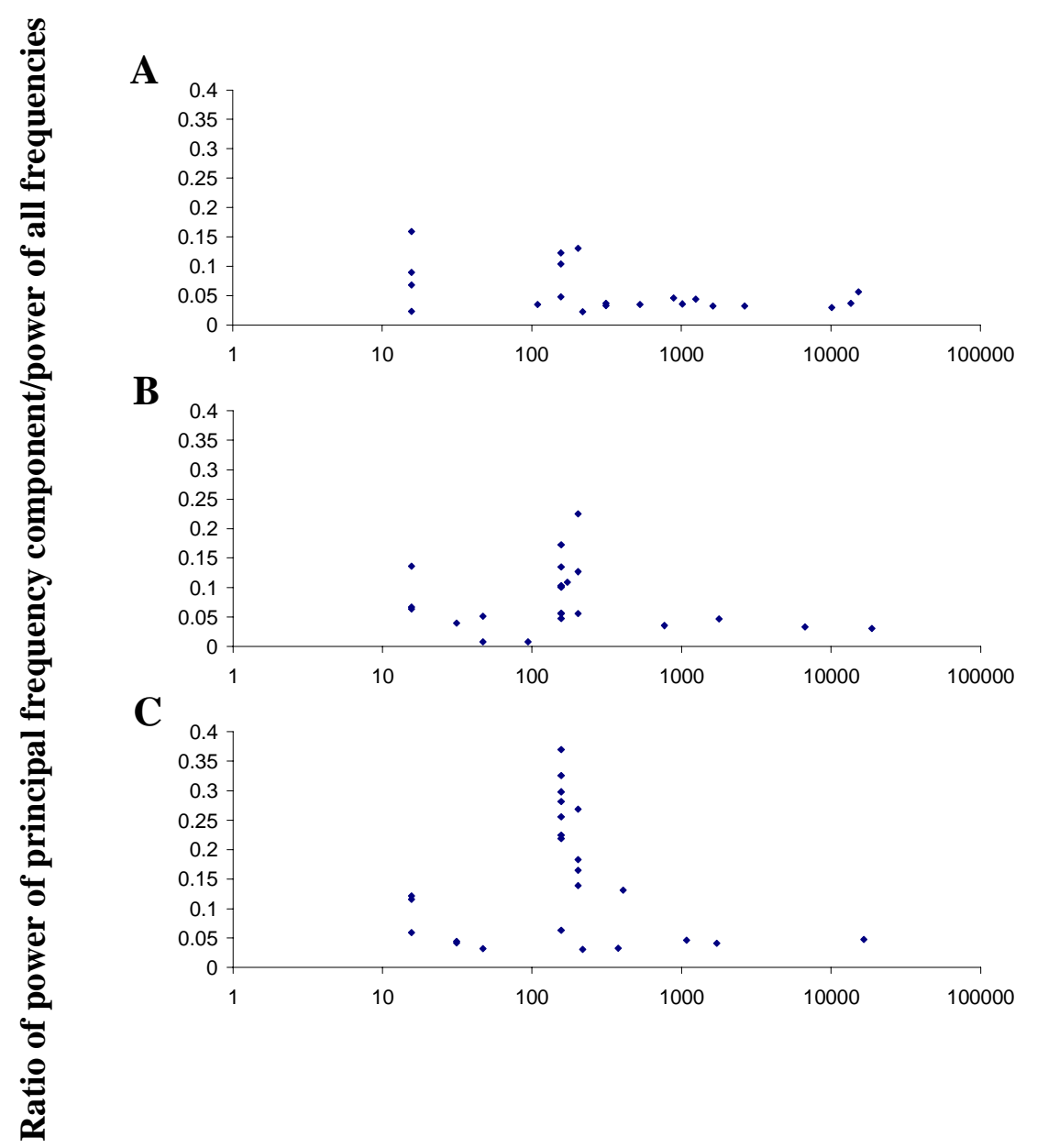

Peak frequency in autocorrelogram $(\mathrm{Hz})$

Fig. 52. Principal frequency components of $\mathrm{DHC}$ responses to $200 \mathrm{~Hz}$ sinusoidal stimulation at 4 amplitudes. A-D, 70, 150, 300 and 500 $\mu \mathrm{m}$. 
Thus, to some degree, the stimulus frequency is reflected in the frequency components of the responding DHCs.

\section{Analysis of firing rates}

Distributions of firing rates for all cells during all combinations of stimulus frequency and amplitude are shown in figs. 53-57. Generally, firing rates increase as a function of both frequency and amplitude, but amplitude has the biggest effect on firing frequency. This is most noticeable at lower frequencies of stimulation. For the stimulus parameters of these experiments, it appears that there is a minimum stimulus amplitude below which there is no response. For most cells, that threshold occurs somewhere between 150 and $300 \mu \mathrm{m}$. Most of the responses recorded during stimuli of less than $300 \mu \mathrm{m}$ aren't very useful. The trends in mean firing rates as a function of stimulus frequency and amplitude are shown in figs. 58-59. There is a positive correlation between frequency and firing rate, as well as amplitude and firing rate. Since the cells respond best at the highest amplitude of each frequency, it is useful to look at the population mean firing rates at each frequency, at the maximal amplitude of stimulation. These rates are shown in fig. 61 . As can be seen in this diagram, the firing rates for the population of all DHCs doesn't significantly increase with increasing frequency. The large error bars are due to the three cells that responded well at frequencies of $50 \mathrm{~Hz}$ and above, and the mean firing rate profile would be relatively flat if these cells were excluded. 
A

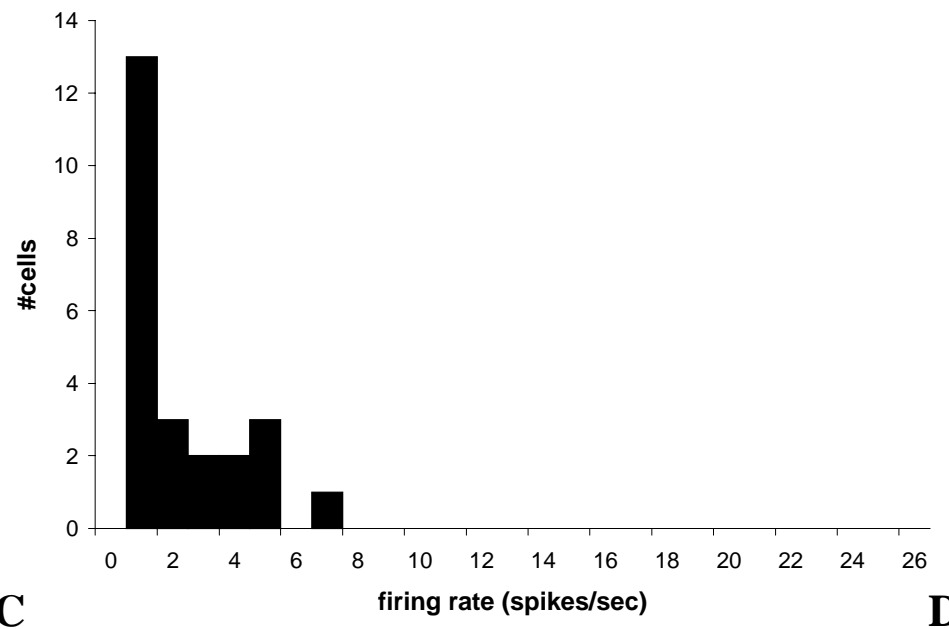

C

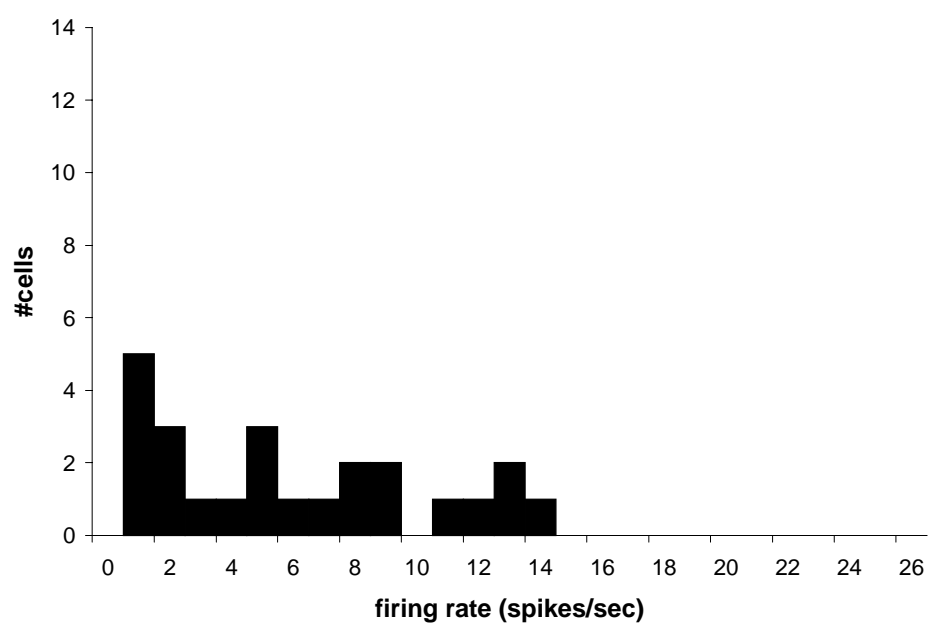

B
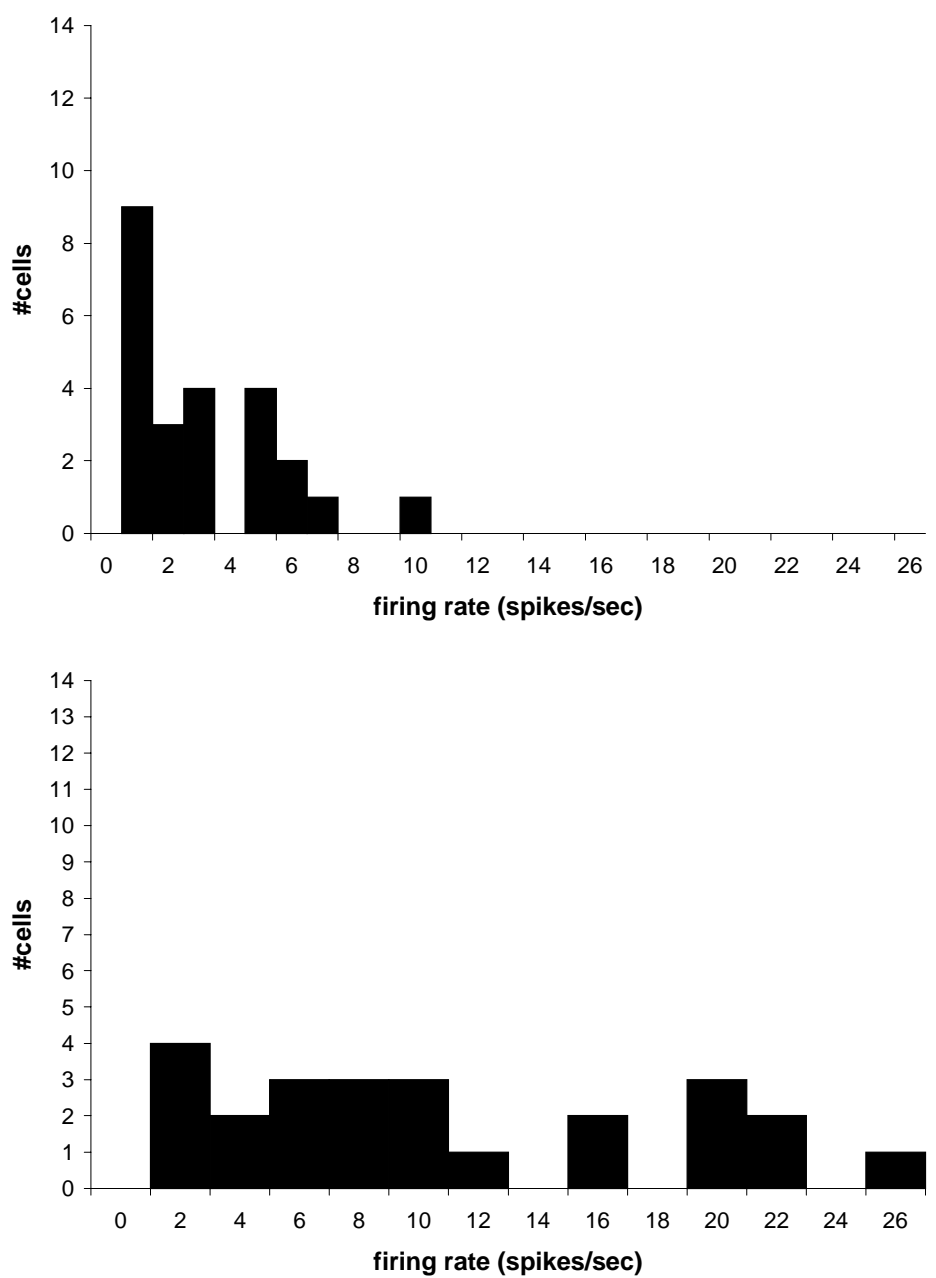

Fig. 53. Summary histograms of $\mathrm{DHC}$ firing rates during $5 \mathrm{~Hz}$ sinusoidal stimulation at 4 amplitudes: A-D, 70, 150, 300 and 500 $\mu \mathrm{m}$. 
A

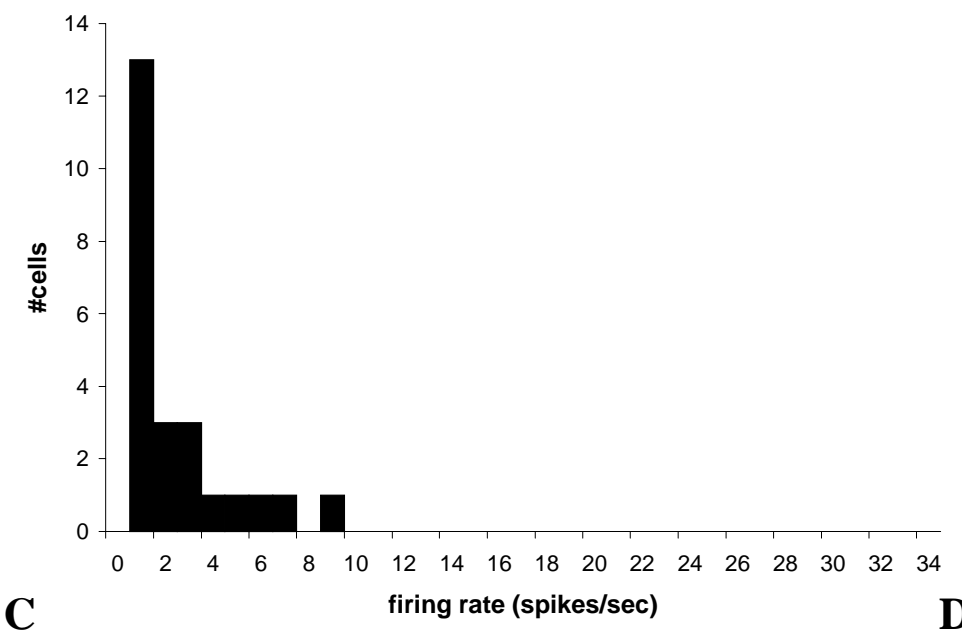

C

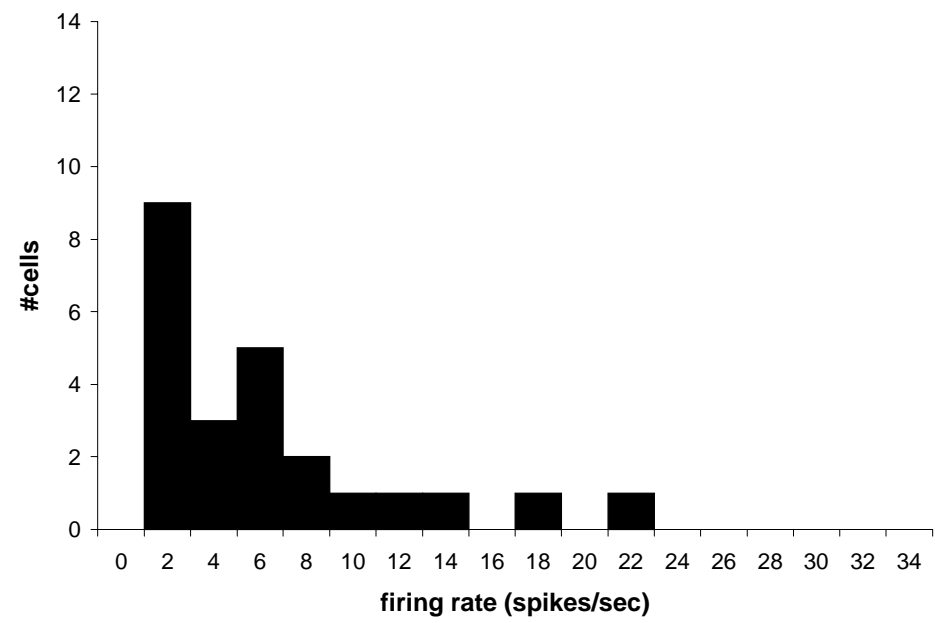

B
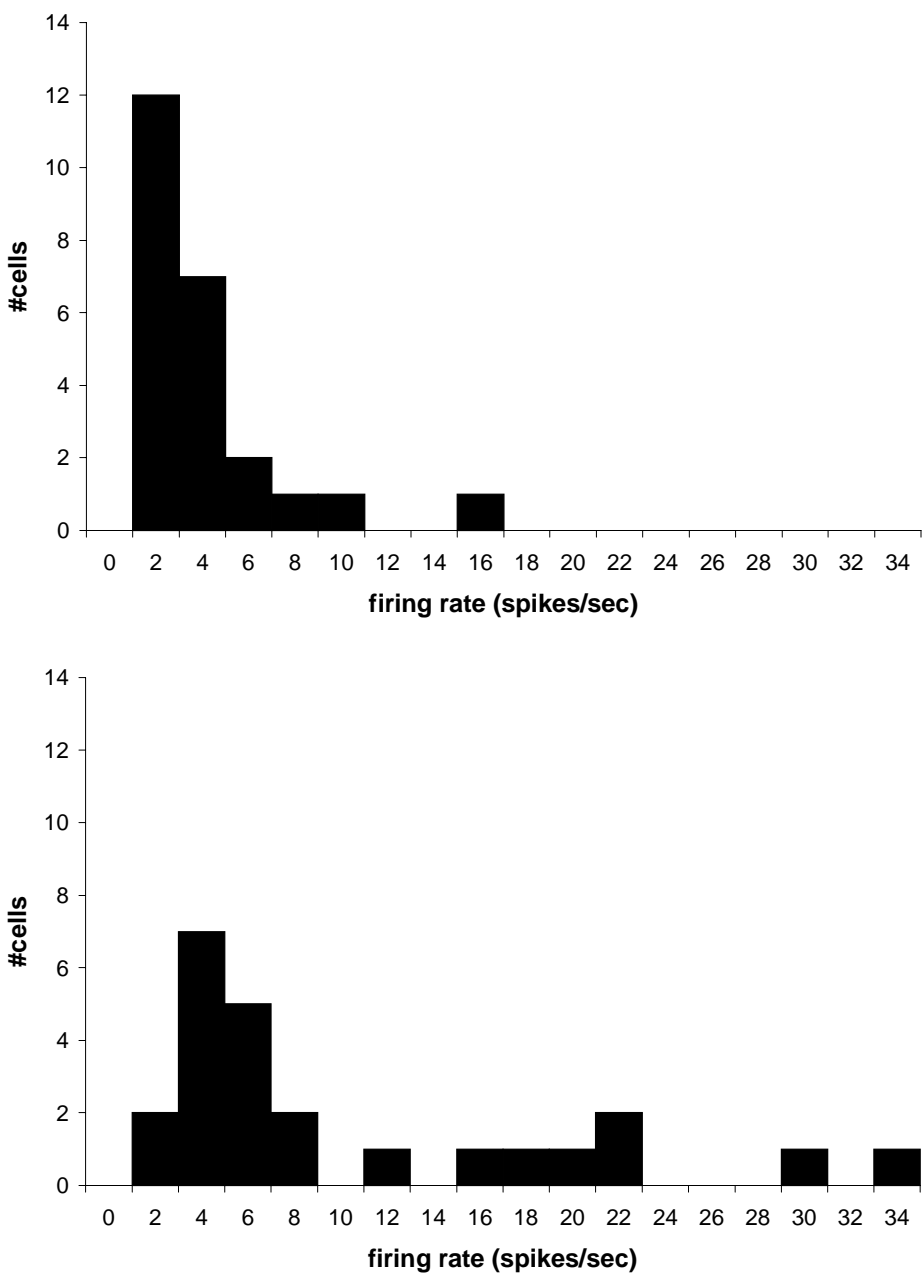

Fig. 54. Summary histograms of $\mathrm{DHC}$ firing rates during $10 \mathrm{~Hz}$ sinusoidal stimulation at 4 amplitudes: A-D, 70, 105, 300 and 500 $\mu \mathrm{m}$. 
A
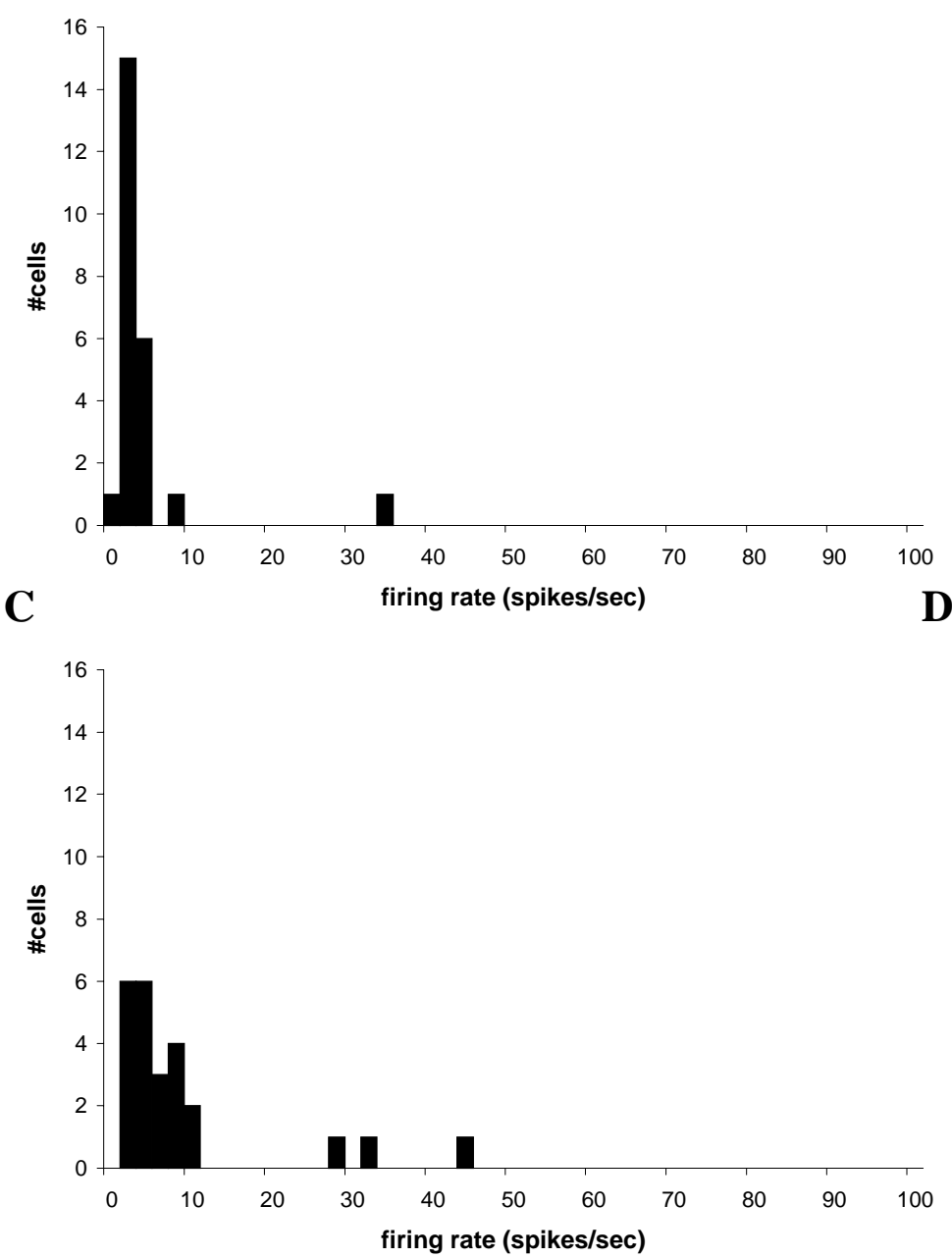

B
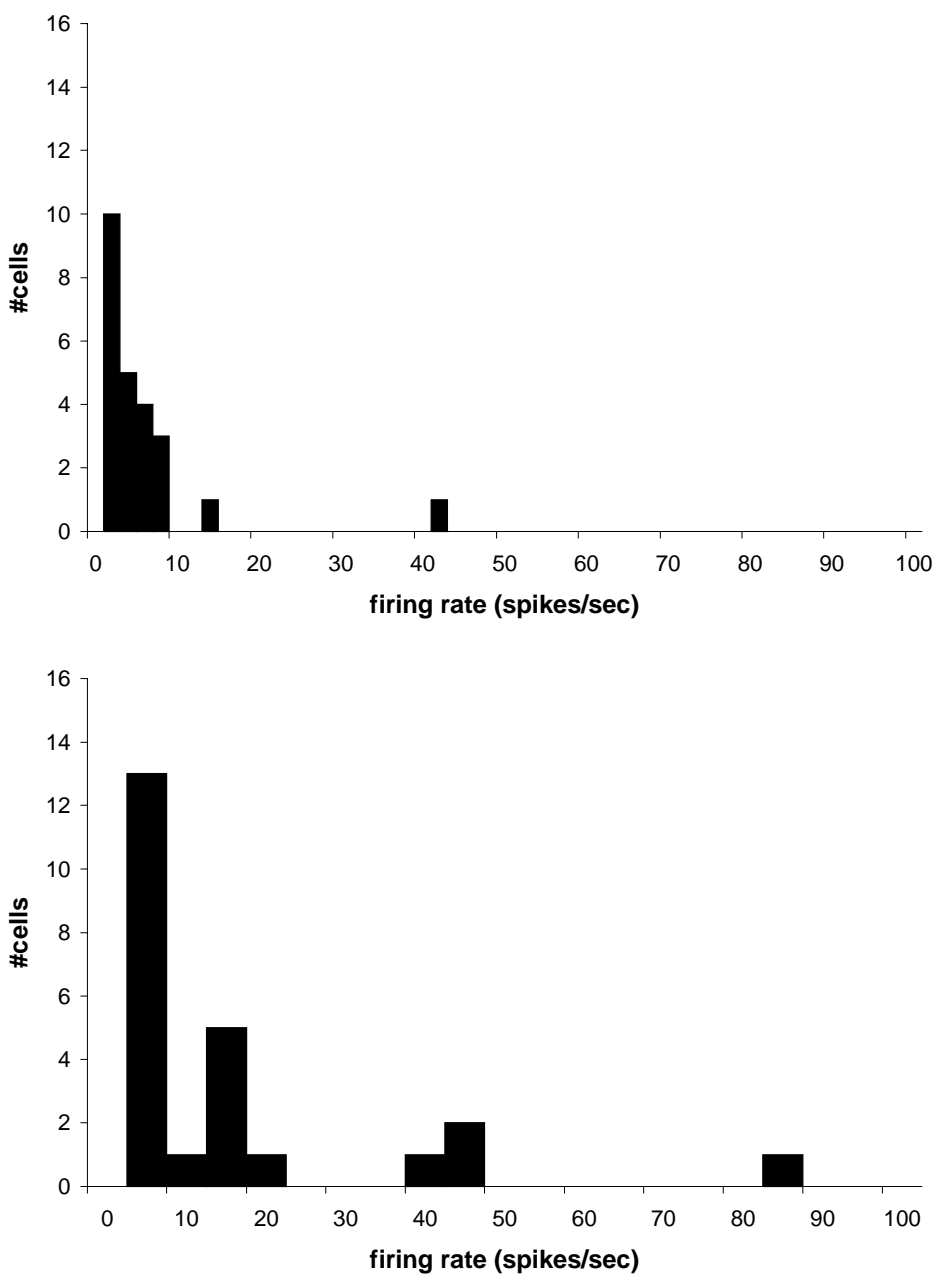

Fig. 55. Summary histogram of $\mathrm{DHC}$ responses to $50 \mathrm{~Hz}$ sinusoidal stimulation at 4 amplitudes: A-D, 70, 105, 300 and 500 $\mu \mathrm{m}$. 
A

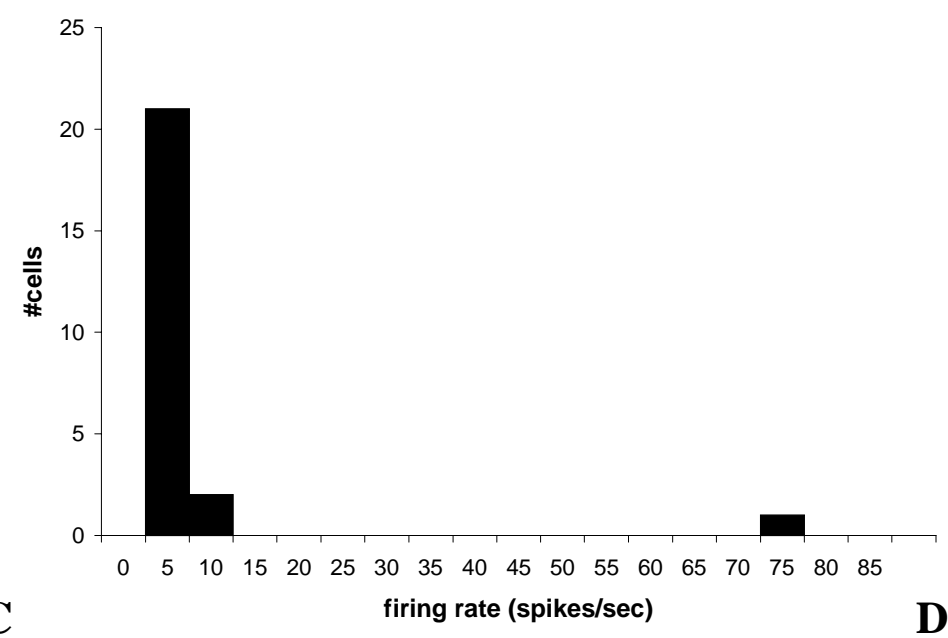

C

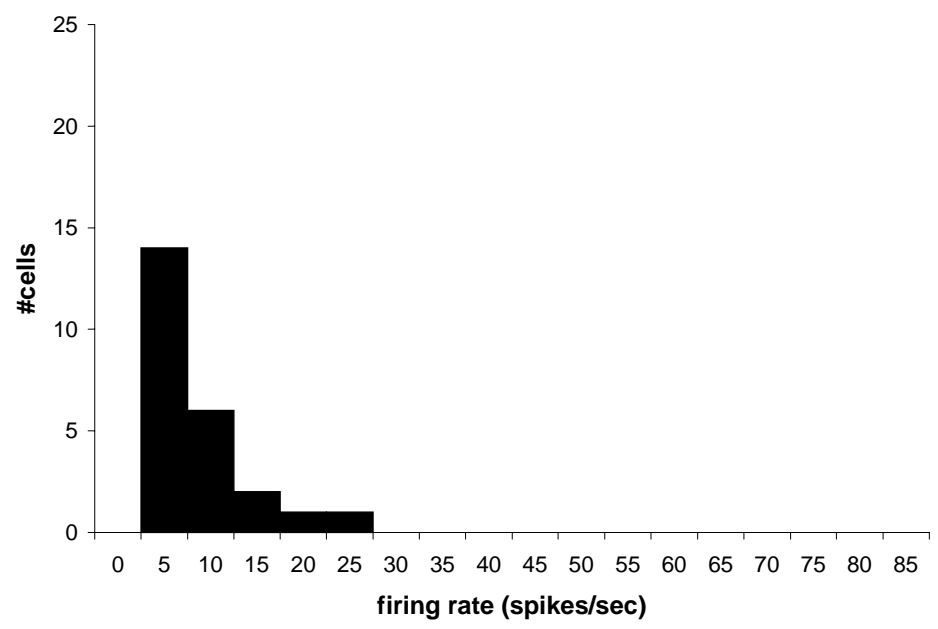

B
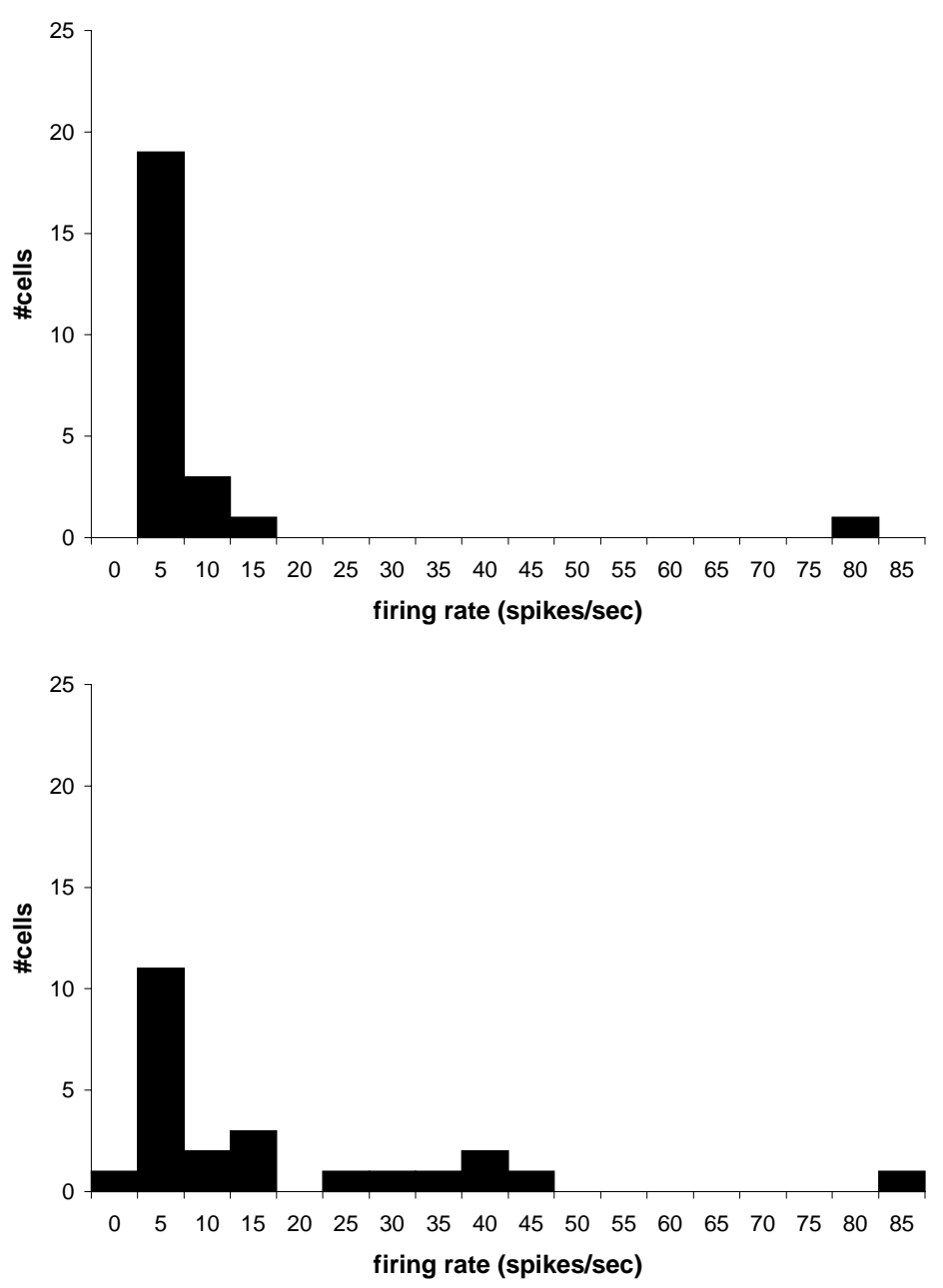

Fig. 56. Summary histogram of $\mathrm{DHC}$ responses to $100 \mathrm{~Hz}$ sinusoidal stimulation at 4 amplitudes: A-D, 70, 150, 300 and 500 $\mu \mathrm{m}$. 
A

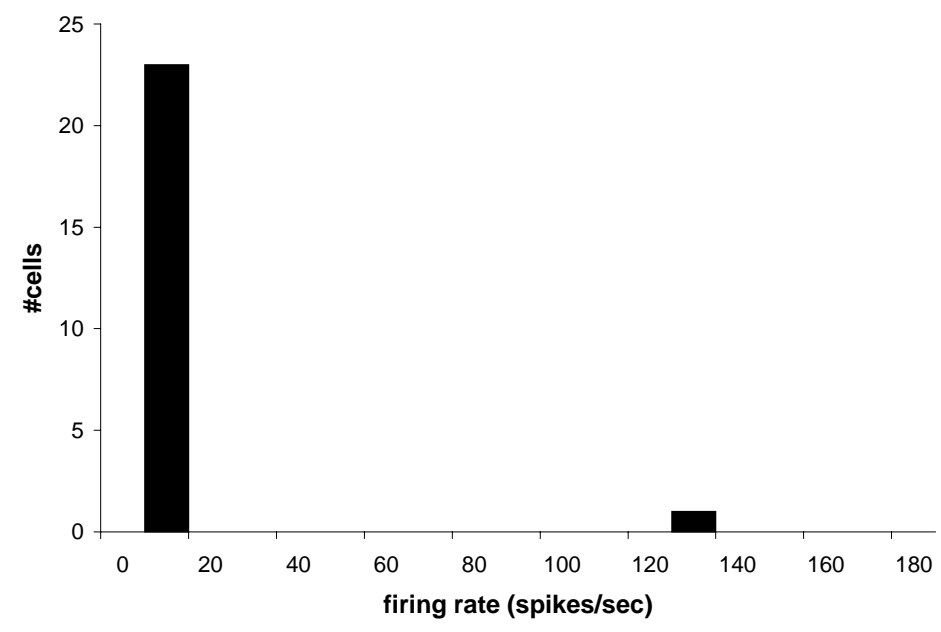

C

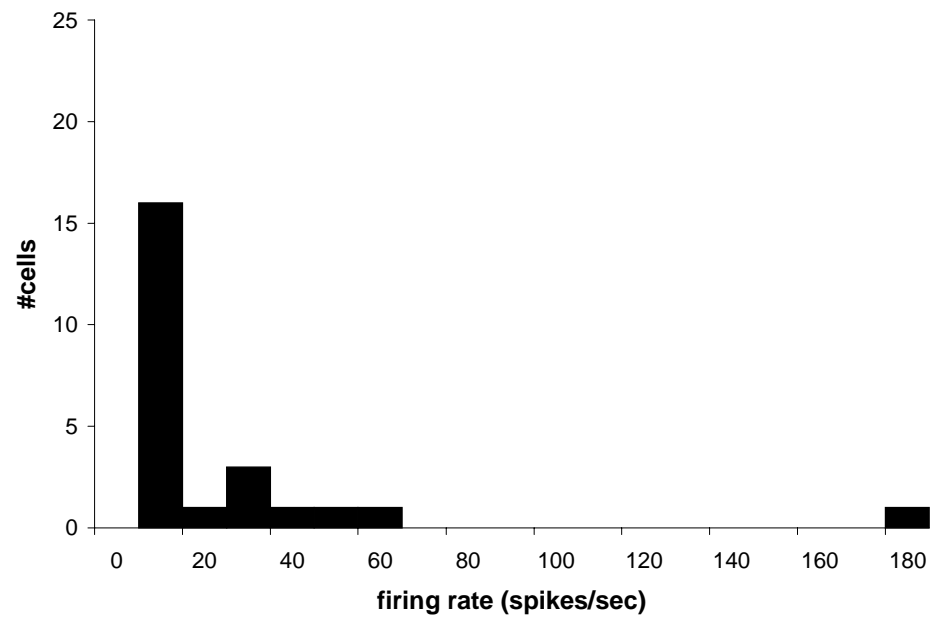

Fig. 57. Summary histograms of $\mathrm{DHC}$ responses during $200 \mathrm{~Hz}$ sinusoidal stimulation at 3 amplitudes: A-C, 70, 105 and $300 \mu \mathrm{m}$.

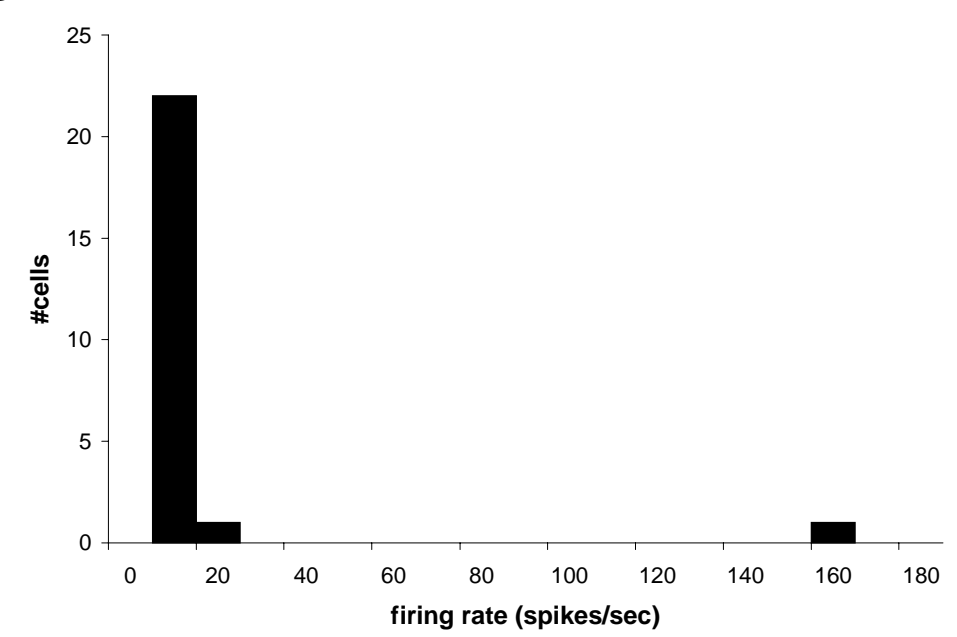




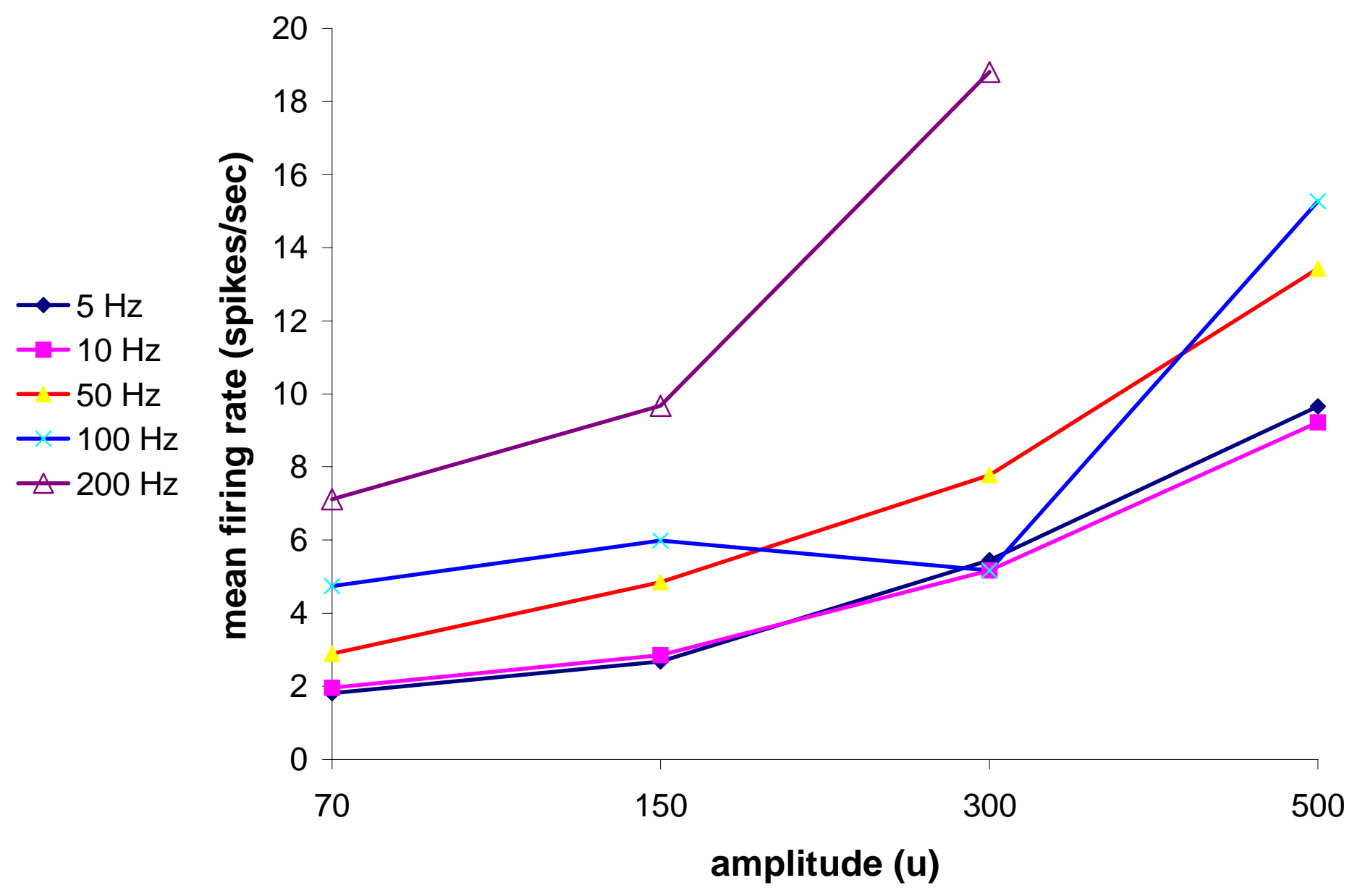

Fig. 58. Firing rate vs. stimulus amplitude at 5 stimulus frequencies. 


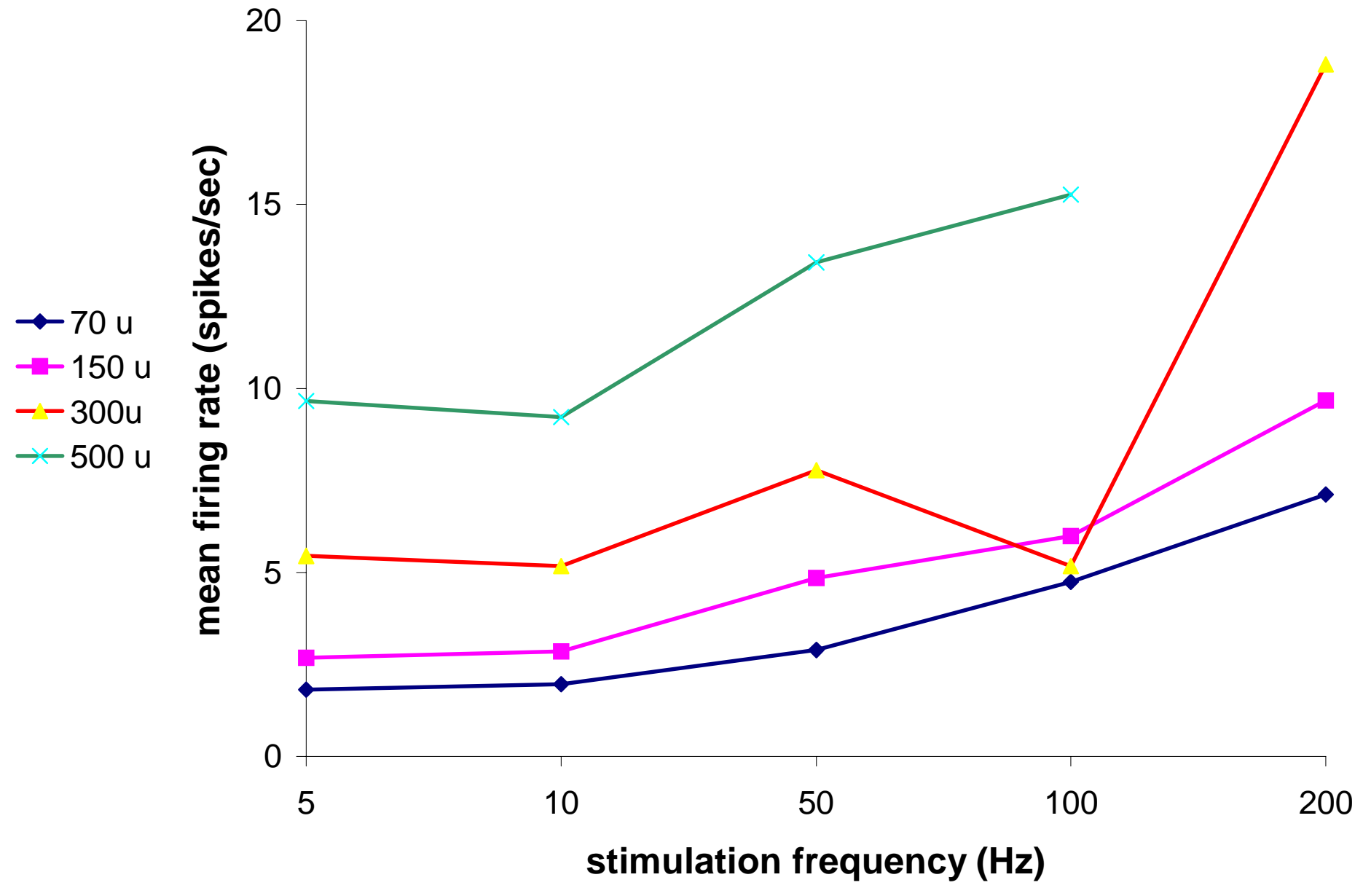

Fig. 59. Mean firing rate as a function of stimulation frequency at 4 amplitudes. 


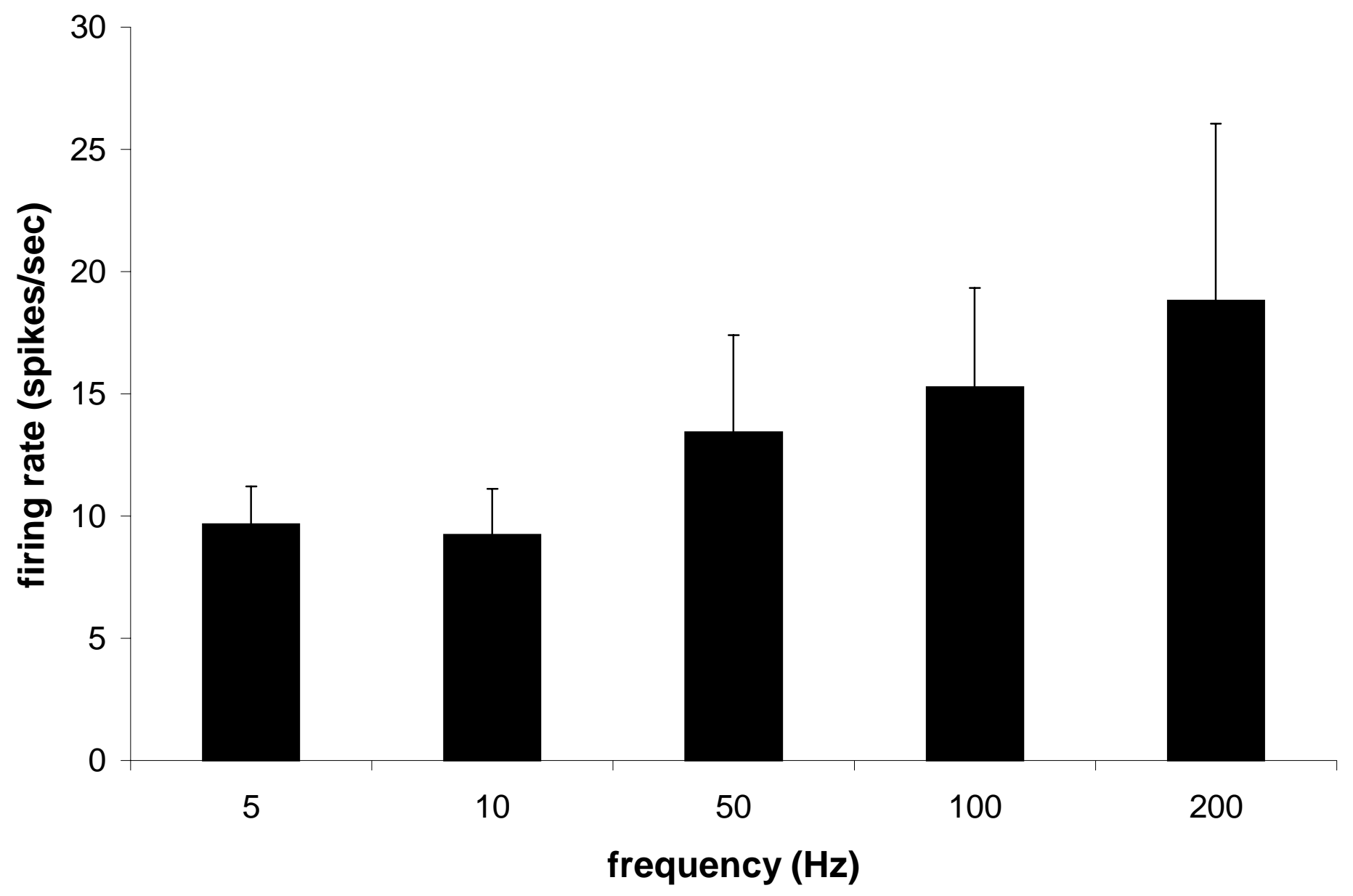

Fig. 60. Mean firing rates at 5 frequencies of highest amplitude stimulation. 


\section{Population responses}

The autocorrelograms of all DHC responses to 5 frequencies of $300 \mu \mathrm{m}$ stimulation are shown in figs. 61-62. The peaks in the autocorrelograms corresponding to the stimulus period are not as pronounced in the mechanically stimulated cell population. This means that there were many more responses that did not correspond with the stimulus period, or that there were not as many responses that occurred at the stimulus period. At 100 and $200 \mathrm{~Hz}$, all but one cell responded with a firing rate appreciably lower than the stimulus frequency. This means that the single cell's responses did not dominate the autocorrelogram in the same way that the several cells did in the electrical stimulation population response. This accounts for the difference in the peak-tobaseline activity in the autocorrelogram. This is reflected in the power spectra shown in figs 63-64. None of the power spectra have principal frequency components that are near the stimulation frequency, even at low frequencies. The frequency of the stimulus and its harmonics are present, but low frequency components dominate. This is probably due to the large constant (DC) component of the combined responses. The principal frequency component of the power spectrum of the mechanically stimulated population is the same as the stimulus frequency when the low frequency peak is removed. Arrows in figs. 63-64 signify the largest component of the response disregarding the low frequencies. 
A

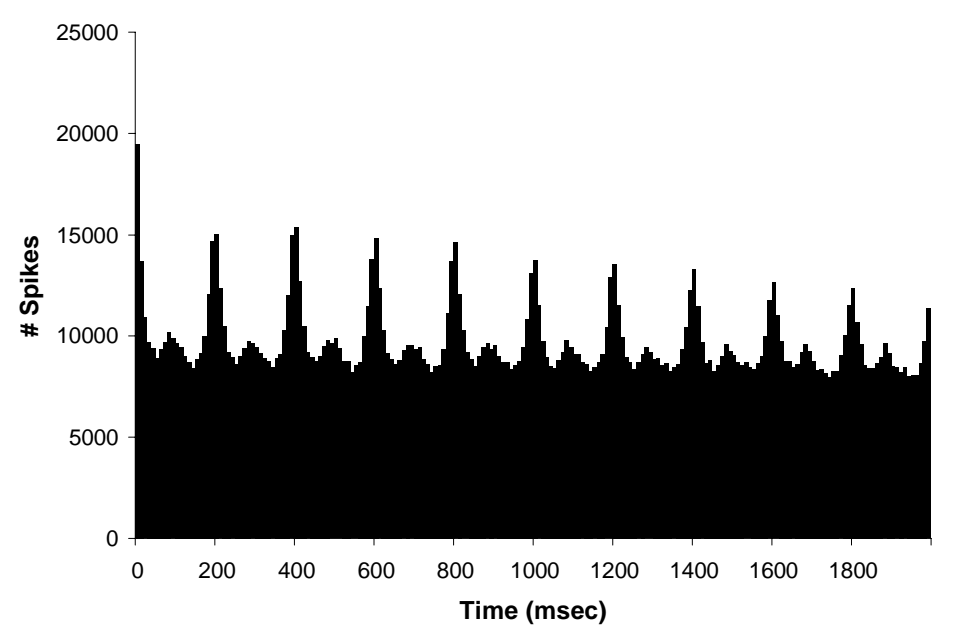

B

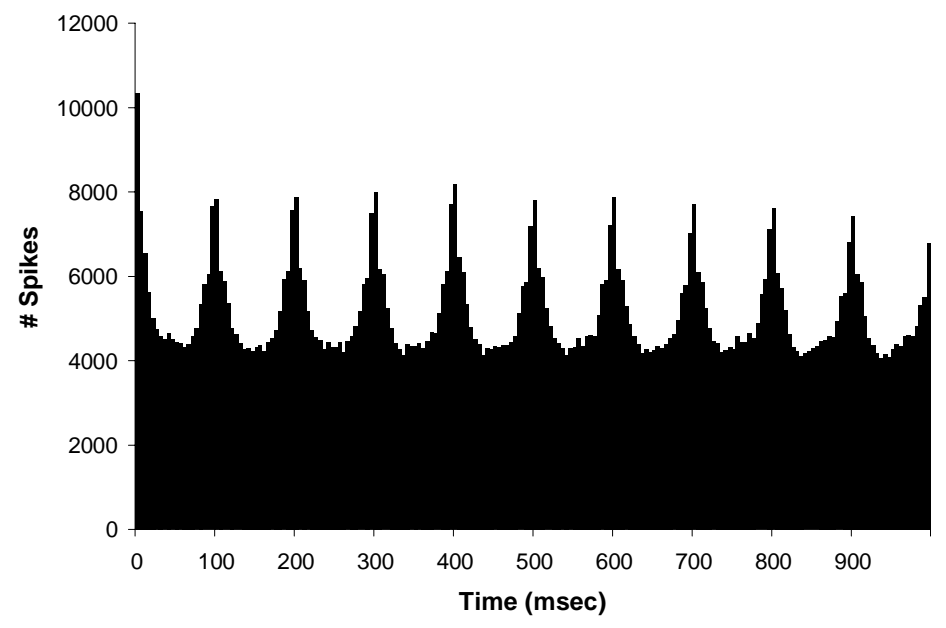

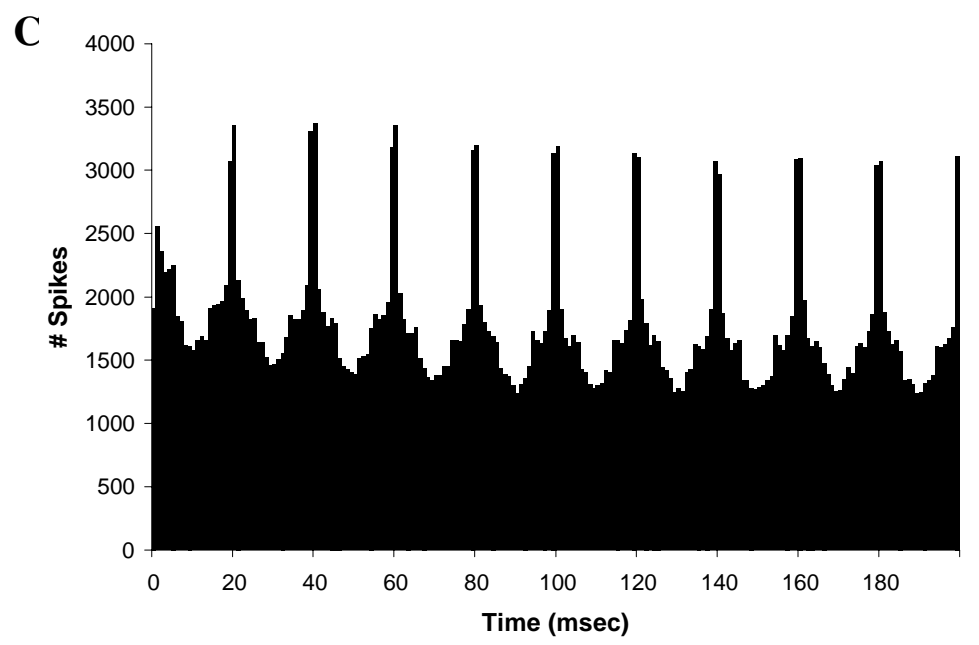

Fig. 61 Autocorrelograms for DHC population responses to 3 frequencies of mechanical stimulation at $300 \mu \mathrm{m}$. A-C, 5,10 and $50 \mathrm{~Hz}$. 
A

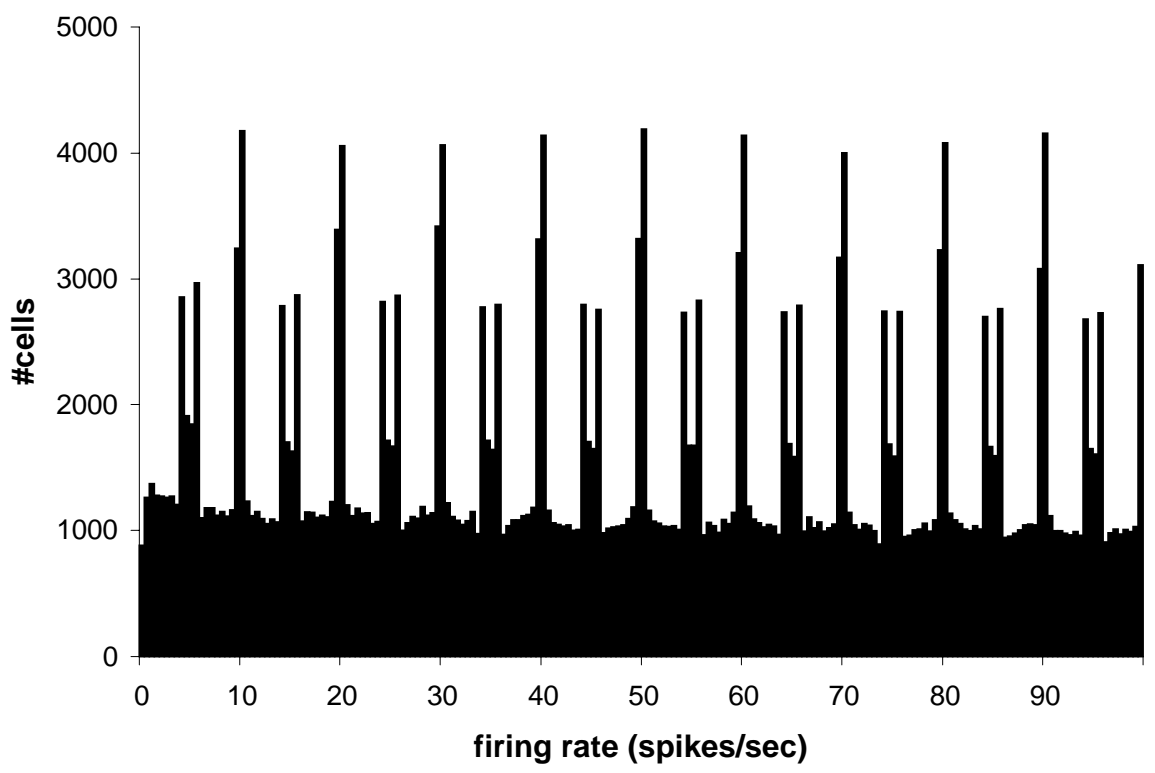

B

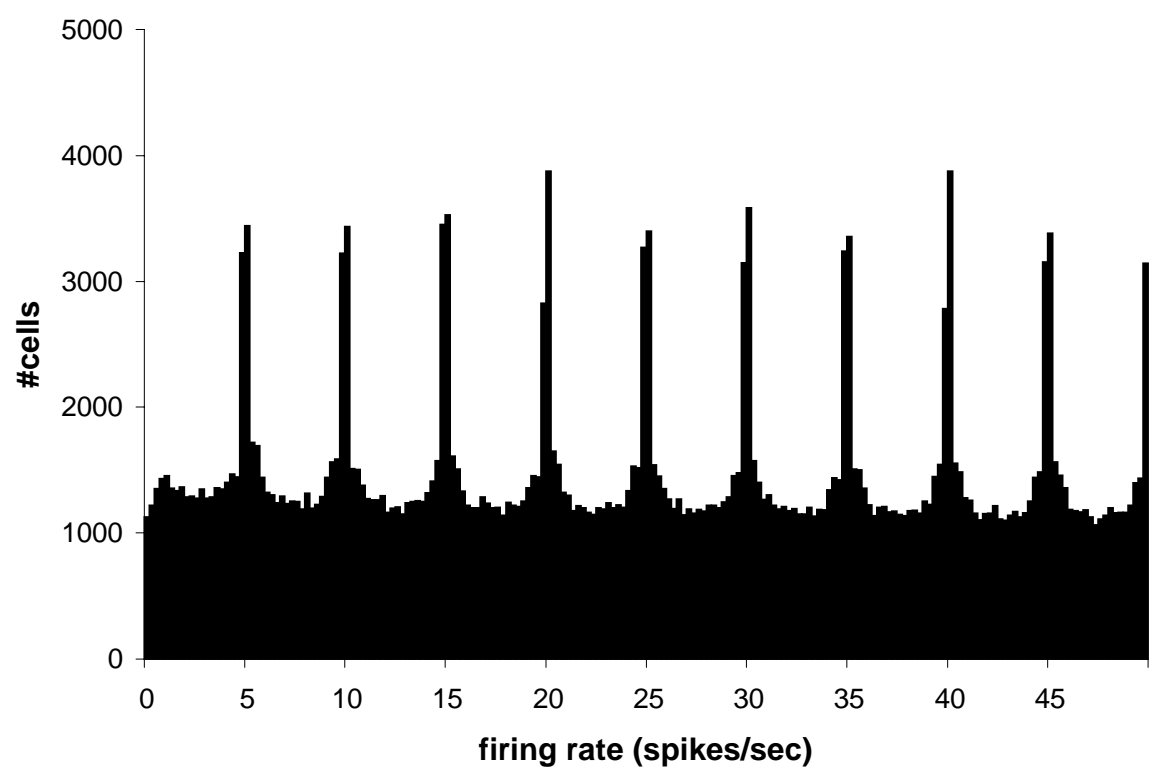

Fig. 62. Autocorrelograms of DHC population responses to two frequencies of $300 \mu \mathrm{m}$ mechanical stimulation at two frequencies. A-B, 100 and $200 \mathrm{~Hz}$. 
A

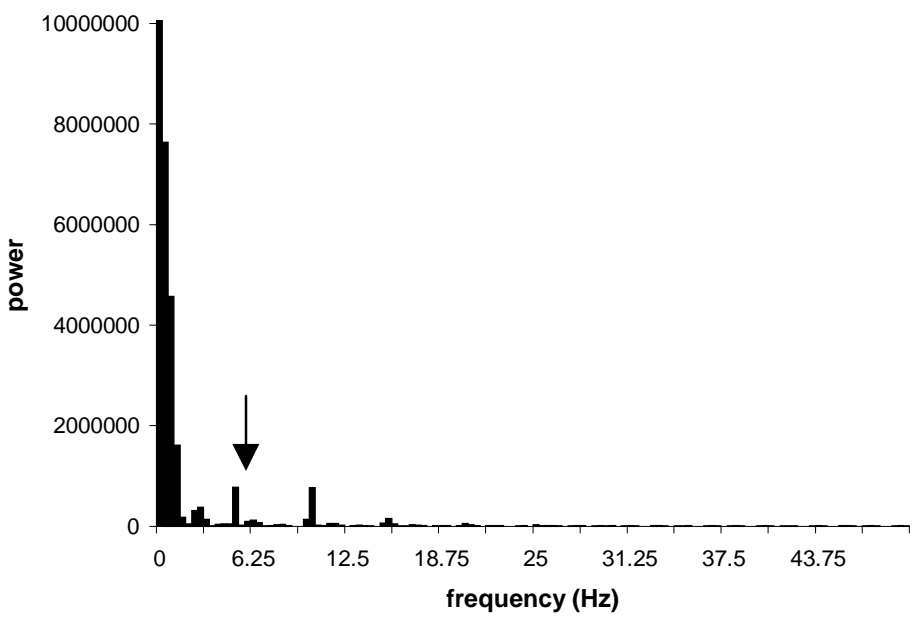

C
B

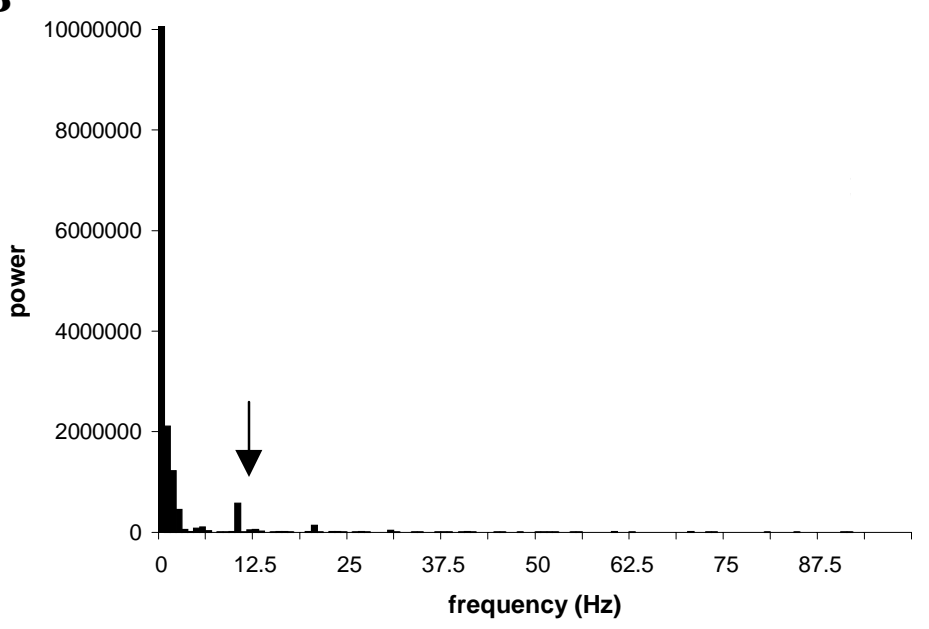

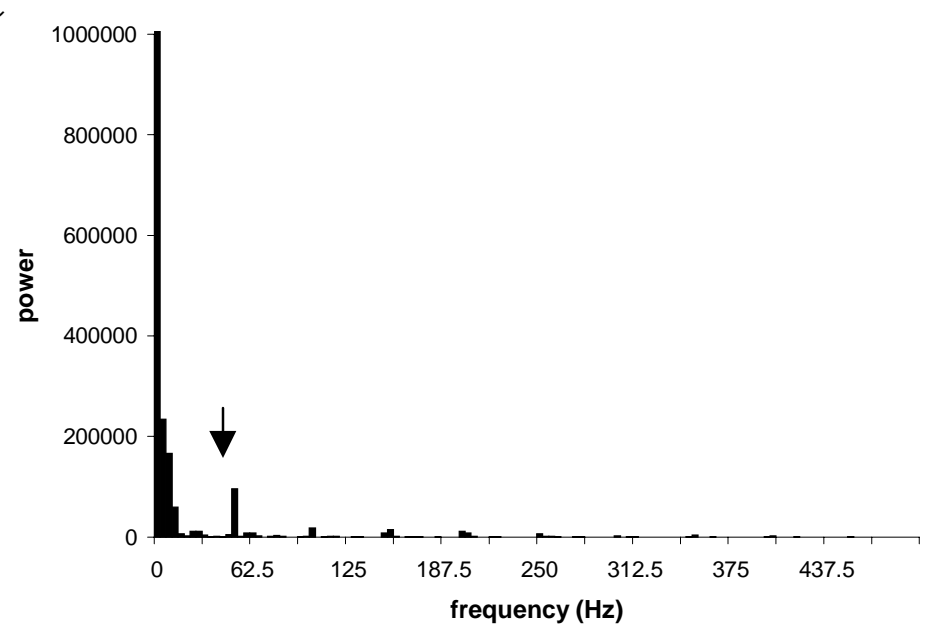

Fig. 63. Power spectra for $\mathrm{DHC}$ population responses to $300 \mu \mathrm{m}$ sinusoidal stimulation at 3 frequencies. A-C, 5,10 and $50 \mathrm{~Hz}$. 


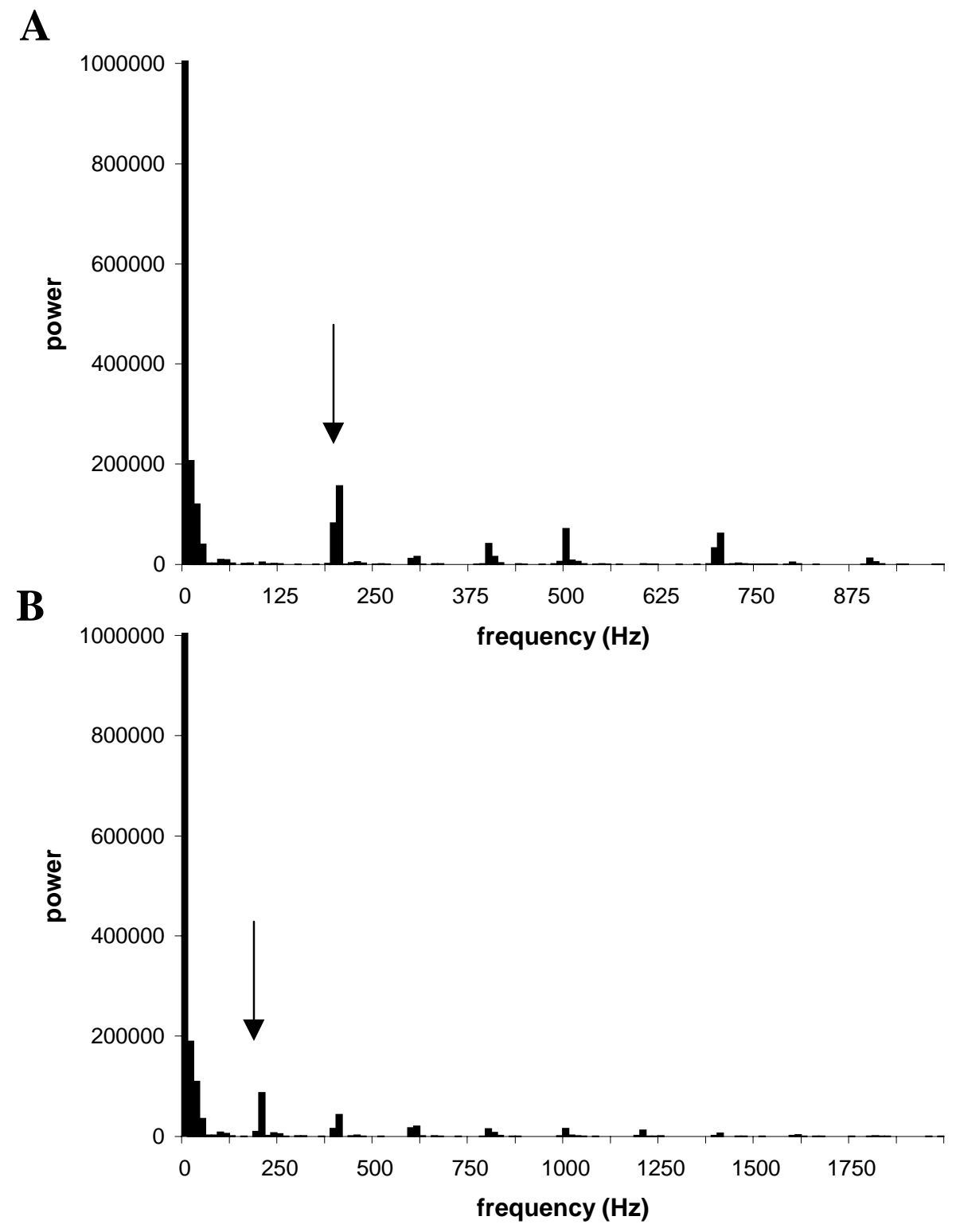

Fig. 64. Power spectra for $\mathrm{DHC}$ population responses to $300 \mu \mathrm{m}$ stimulation at two frequencies. A-B, 100 and $200 \mathrm{~Hz}$. 


\section{Effects of adaptation}

Vector strengths and firing rates during the first 10 cycles of sinusoidal stimulation are shown in figs. 65-68. Vector strengths and firing rates increase with increasing stimulus amplitude. With increasing stimulus frequency, firing rates go up, and vector strengths go down. As the number of spikes goes up with increasing frequency, apparently their timing relative to the phase marker becomes more and more dispersed.

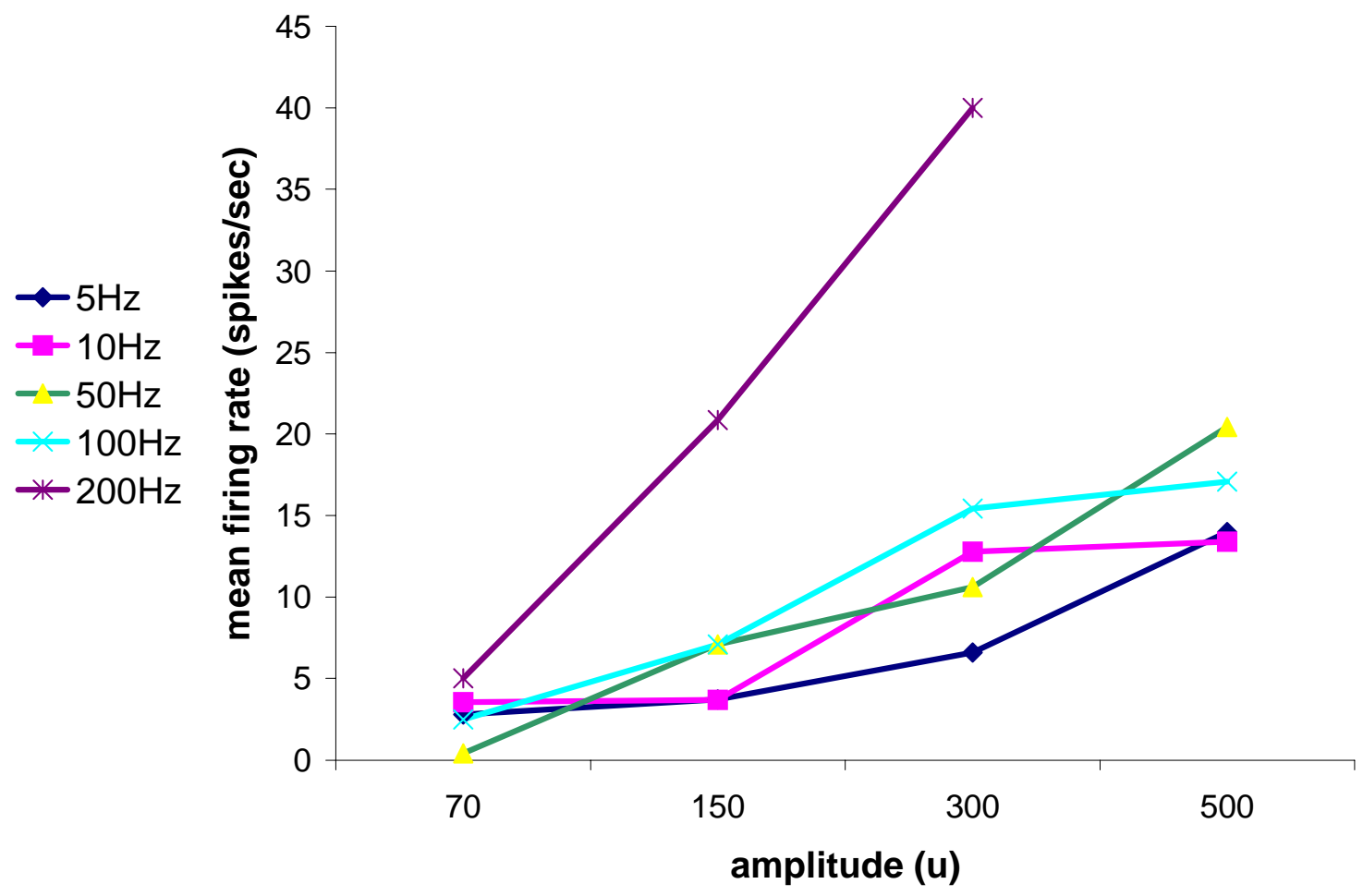

Fig. 65. Effect of increasing amplitude on mean firing rate during the first 10 cycles of stimulation at four amplitudes. 


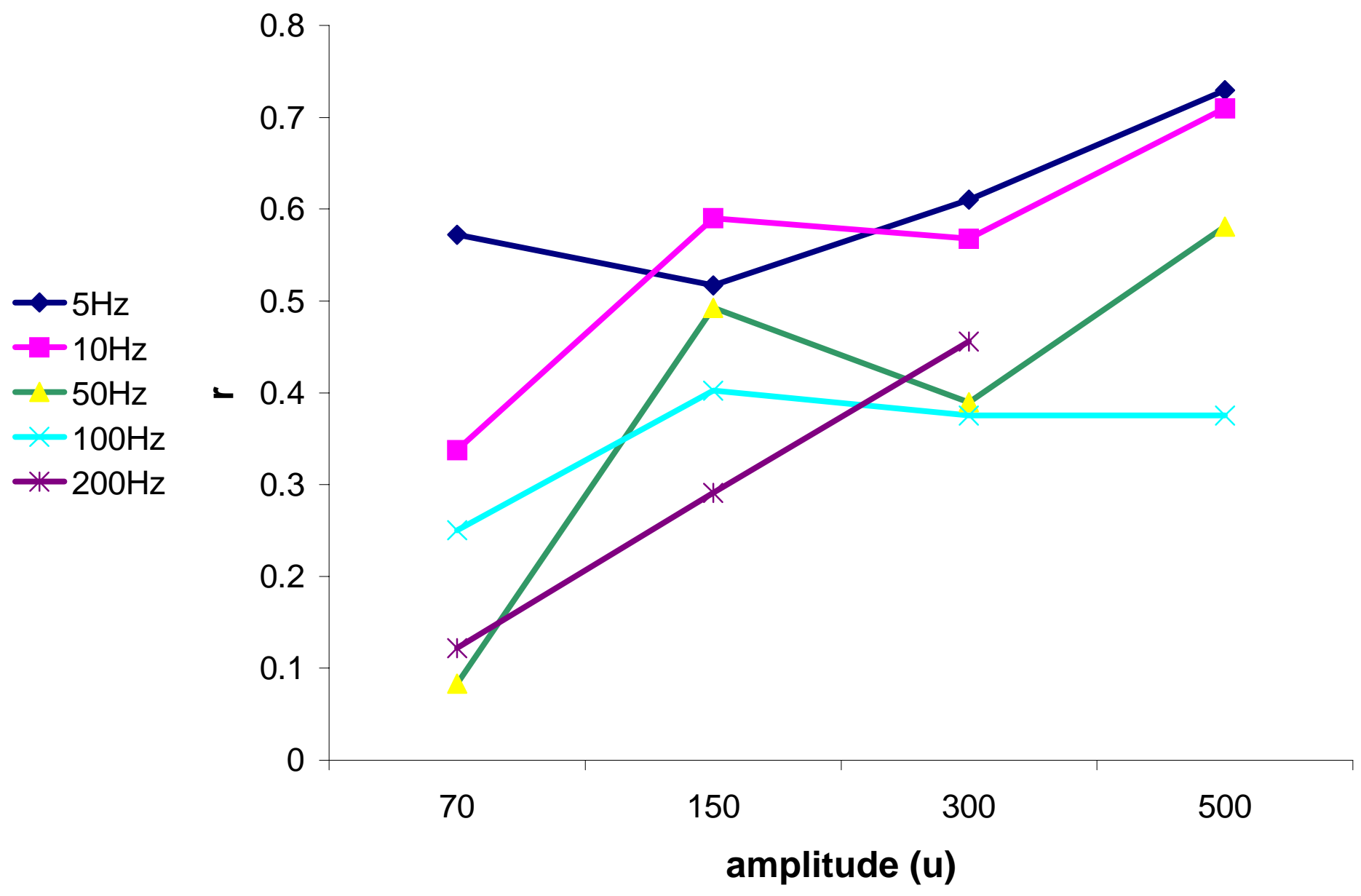

Fig. 66 Effect of increasing stimulus amplitude on vector strength during the first ten cycles of stimulation. 


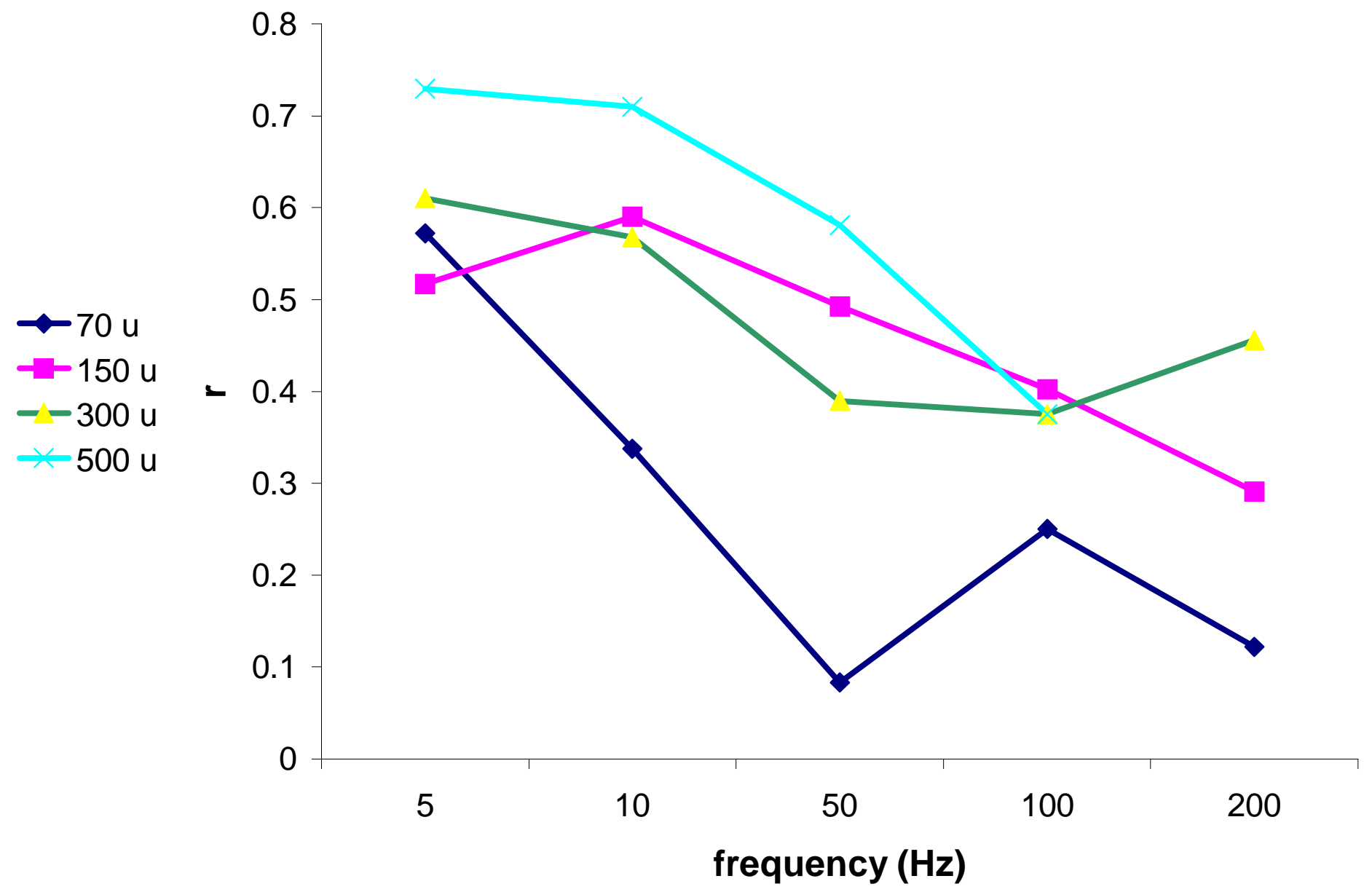

Fig. 67. Effect of increasing stimulus frequency on mean vector strength. 


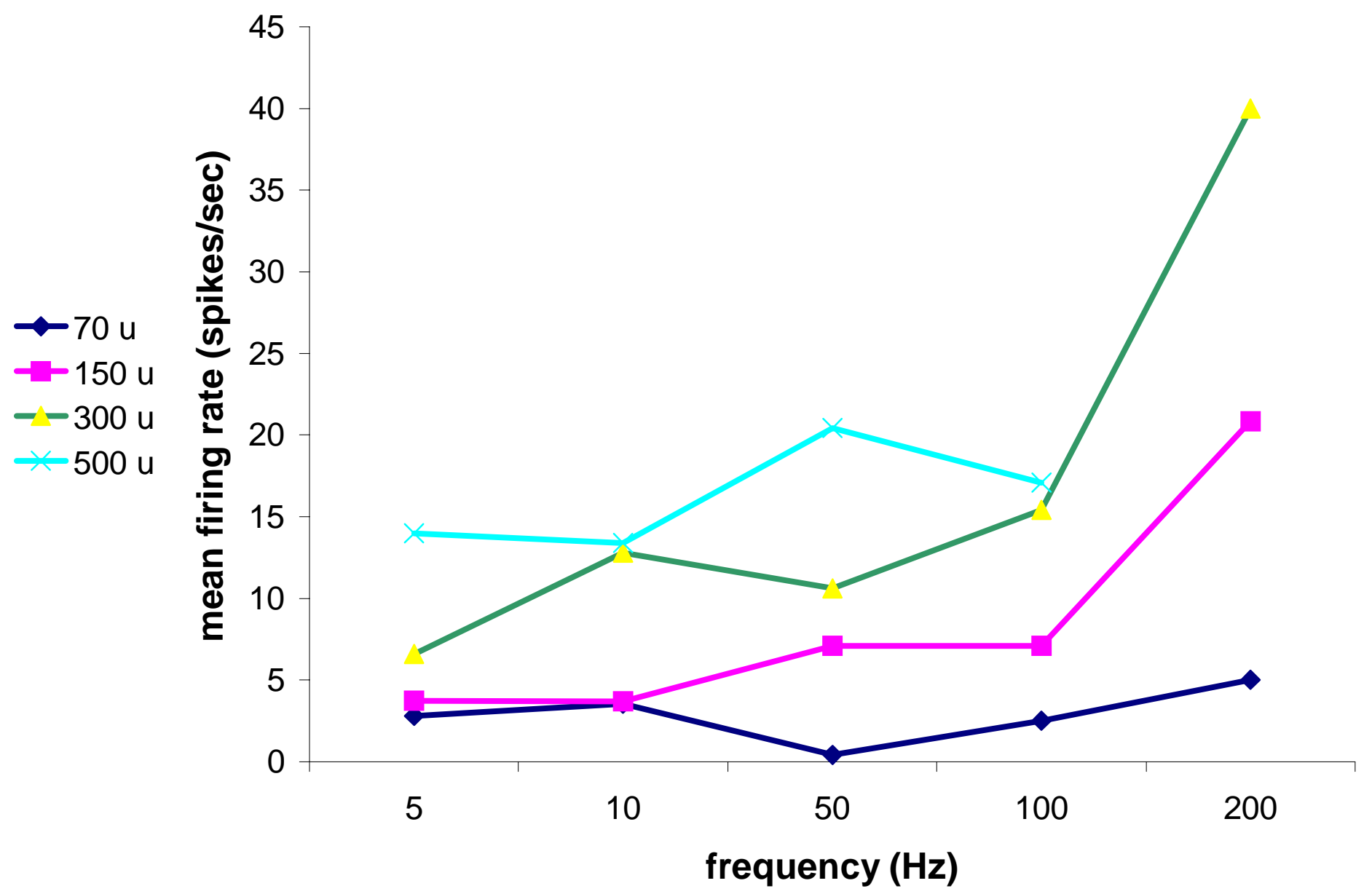

Fig. 68. Effect of increasing stimulus frequency on mean firing rate during the first ten cycles of stimulation. 


\section{Discussion}

In the study of sensory systems, it is important to identify the anatomical structures that contribute information regarding a particular sensory modality to the brain. Once the sensory system is at least partially described, the individual elements of the system can be studied with regard to their encoding of particular aspects of sensory stimuli.

The encoding of vibrotactile pitch has been studied extensively in the somatosensory cortex, and in most non-spinal subcortical somatosensory nuclei. In these studies, aspects of the discharge patterns of single neurons were used to examine possible strategies the CNS uses to encode tactile pitch. Most of the studies focused on two main aspects of the elicited spike trains: firing rate and periodicity. The brain receives no direct information about the stimulus, only the representation of the stimulus embedded in the spike trains of ascending neurons.

Mountcastle (1990) asserted that periodicity, not firing rate, is the important variable in cortical spike discharges to encode vibrotactile pitch, since no significant difference was seen in the firing rates of cortical neurons of monkeys performing a same-different vibrotactile discrimination. It has been suggested that the periodicity of the spike discharge in response to vibrotactile stimuli are important for giving an organism the perception of an even vs. an uneven stimulus (i.e. a periodic discharge in a sensory neuron contributes to a percept of an even stimulus, whereas an aperiodic discharge gives the percept of a 'choppy' stimulus). (Reviewed in Johnson, 2000). 
The significance of firing rate on the perception of vibrotactile stimuli has been examined recently by Romo's group (Romo et al., 1998). In these experiments, current pulses were applied to cortical neurons whose RFs were defined on the skin. The animals had been previously trained in a vibration frequency discrimination task in which two stimuli were applied, and the animals determined whether the second applied stimulus was higher in frequency than the first. During the experiments, the monkeys compared a first mechanical stimulus with a second and decided if the second was higher than the first. Next, the animals were subjected to a mechanical stimulus first, followed by a train of current pulses to the somatosensory cortex in the area corresponding to the area of skin being mechanically stimulated. The current pulses were of the same mean frequency as the mechanical vibration, but were aperiodic. The animals were able to do the discriminations, showing that periodicity is not necessary for the encoding of vibrotactile pitch. Therefore, firing rate may be important in frequency coding of stimuli necessary for same-different vibrotactile discrimination in monkeys.

The objective of these studies was to determine whether or not there are neurons present in the dorsal horn that are able to encode aspects of temporal skin stimuli in either the periodicity or rate of their discharges. Several neurons were found that can encode vibrotactile pitch in their firing rates, and most were able to encode the stimuli in the periodicity of their discharge. Approximately $5 \%$ of all of the neurons studied were able to encode high frequency $(>=100 \mathrm{~Hz})$ stimuli in both the periodicity and rate of their discharges. In a cortical study (Lebedev and Nelson, 1996), the same percentage of cells $(5 \%)$ were able to follow a $127 \mathrm{~Hz}$ mechanical stimulus. Most studies of the 
encoding of vibrotactile pitch are designed to study only those neurons capable of encoding high frequency vibration in their discharges.

In this group of studies, no assumptions were made regarding the importance of dorsal horn in the transmission of vibrotactile pitch information that reaches consciousness. The neurons in these experiments were unidentified as to projection targets, so they could in principle all be local interneurons. These studies were conducted to determine whether or not neurons in the dorsal horn could respond to periodic cutaneous stimuli in a manner similar to that reported in other subcortical somatosensory structures, and in cortical neurons. Romo (1998) has shown that some cortical neurons in the somatosensory cortex contribute directly to perceptions in monkeys performing a vibrotactile discrimination task. These were the first studies that showed the importance of individual central somatosensory neuronal firing patterns in discrimination tasks. In other levels of the somatosensory system (e.g. thalamus and dorsal column nuclei), the role of single neurons with regard to vibrotactile perception is unknown. The subcortical levels of the somatosensory system have been designed to analyze spike trains in a way similar to those used in studying the cortex. The responses of DHCs to periodic electrical and mechanical stimuli were quantified using methods similar to those used in other areas of the somatosensory system.

\section{A. Firing rate as a possible code for vibrotactile pitch}

The majority of cells in these experiments were able to encode stimuli with firing rate changes at low stimulus frequencies of either 5 or $10 \mathrm{~Hz}\left(\mathrm{~s}^{-1}\right)$. In the electrical 
stimulation paradigm, most cells were able to fire at rates greater than the stimulus frequency up to $10 \mathrm{~s}^{-1}$. At 50 and $100 \mathrm{~s}^{-1}$, firing rate is often negatively correlated with stimulation rate. All but 4 cells were unable to respond well to the $100 \mathrm{~s}^{-1}$ stimulus, and most firing rates in response to the $100 \mathrm{~s}^{-1}$ were less than 5 spikes/sec. The average firing rates at this stimulation frequency are dominated by the rates of these four cells. However, this does show that there are at least some DHCs that are able to respond once per stimulus presentation at higher $\left(50 \mathrm{~s}^{-1}\right.$ and higher) stimulation rates. The cells that were able to respond at high stimulus rates did not seem to be 'tuned' to high frequency stimulation, because they responded well at low stimulation rates as well. The RFs of these cells were all on hairy skin, so there appears to be no correlation between the ability to follow high frequency stimuli and the difference between glabrous/hairy skin. Two of these cells were located in lamina III, one in lamina IV and one was not reconstructed, indicating that they do not come from a specific area of the dorsal horn, but could possibly be confined to hairy skin.

$\mathrm{DHC}$ responses to mechanical stimulation differed from responses to electrical stimulation, especially at low mechanical amplitudes. The amplitude of the electrical stimulus was increased until a driven response was obtained, which means that the stimulus probably was eliciting spikes in many primary afferents. This would result from electrotonic spread of the stimulus current away from the stimulus site due to increasing stimulus voltage. Since the fibers are being stimulated directly (not through their peripheral receptor structures), the electrical stimulus provided DHCs with a much larger synchronous input, although conduction velocity differences could theoretically play a role. Since DHCs are connected to more than one type of primary afferent 
(Tapper et al., 1973), it is possible that a DHC will not respond well to a mechanical stimulus of a given frequency and amplitude simply because it is not connected to receptors that are adequate for transducing that stimulus. Although the electrically characterized cells responded more robustly (i.e. higher vector strengths and firing rates) than the mechanically characterized ones, the electrical paradigm is really an artificial situation. The results can be thought of as approaching the upper limit of cells' ability to encode periodic stimuli. In the mechanical stimulation, transduction of skin movement by cutaneous receptors caused spikes in primary afferent fibers, and it is likely that the numbers of stimulated afferents was smaller than those recruited by the electrical stimulus. Electrical stimulation primarily engages hair follicle axons (Brown et al., unpublished), very precisely synchronized with each other, at 1:1 entrainment. Mechanical stimulation engages a variety of mechanoreceptor types much less synchronously, and probably not at 1:1 entrainment for the lower amplitudes and higher frequencies.

Firing rates in mechanically stimulated DHCs were very low at low displacement amplitudes, and increased with increasing stimulus amplitude. This could be due to the recruitment of a larger number of primary afferent fibers. The increase in $\mathrm{DHC}$ firing rate could be due to the fact that the number of fibers responding doesn't change, but each fiber is firing more often as it nears its threshold for mechanical stimulation. It is likely that both occur. It is important to note that nearly all of the cells required a preindentation to respond at all. This is probably because mechanical coupling between the skin and the stimulus was insufficient at the zero indentation position. As the preindentation is increased, the force of the stimulation increases because the receptor 
structures will be mechanically deformed more. The actual amount of force delivered will also depend on the type of tissue underlying the stimulated DHC RF(e.g. skin overlying bone will provide more mechanical coupling that skin overlying muscle). At larger stimulus amplitudes, the DHC responses were qualitatively similar to the electrical responses. There is a positive correlation between firing rate and stimulus frequency at 5 and $10 \mathrm{~Hz}$, but the responses show no uniform trend at higher frequencies. Only one cell was able to follow higher frequencies (100 and $200 \mathrm{~Hz}$ ) with a firing rate that was near the stimulation frequency. Several other cells increased their firing rates during higher stimulation frequencies, but only one had a discharge rate that was greater than half the stimulation frequency. This cell did appear to be tuned, as it responded poorly at low frequencies even at high amplitudes ( 5 and $10 \mathrm{~Hz}$ at $500 \mu)$. At stimulus frequencies of $50 \mathrm{~Hz}$ and up, this cell had firing rates that were not as high as the stimulation frequency, but they were within $10 \%$. Once again, there were not many cells that could encode higher frequencies of vibration, but at least one was able to encode vibrotactile pitch in its discharge rate.

The small numbers of cells that can respond well to high frequencies of vibration could be a manifestation of several possibilities regarding the importance of DHC coding of pitch via firing rate. One possibility is that there is a small group of DHCs that respond in a nearly $1: 1$ fashion to at least $200 \mathrm{~Hz}$. One cell of $24(4 \%$ of the sampled population) in this study responded this way. There is no way to comment on how many of these types of cells exist. Another possibility is that the dorsal horn does not use firing rate to represent vibrotactile pitch. It is also possible that the majority of DHCs simply cannot encode vibrotactile pitch at all. The dorsal horn does have a few 
neurons that can encode high frequency periodic stimuli in their firing rates, and these neurons respond in the same fashion as dorsal column, thalamic, and sensory trigeminal cells, at least to $100 \mathrm{~Hz}(200 \mathrm{~Hz}$ for the mechanical stimulation). This shows that the dorsal horn may be involved in the pathway for transmitting vibrotactile pitch to the brain, although it is not known if these neurons contribute to perception (as is the case for dorsal column nucleus neurons, for example).

An interesting aspect of discharge rate is that firing rate increases need not necessarily encode vibrotactile pitch. In fact, it has been shown that in some primary afferents (SA1), a rate code is used for intensity, not pitch (Vallbo et al., 1984). It is possible that the increase in firing rate could be perceived as pressure intensity. Since DHCs receive input of mixed submodality, it would seem likely that afferents that respond well to higher frequencies of vibration would dominate the input to the responding DHCs.

Firing rate is a viable coding strategy for the majority of neurons in these studies.at stimulus frequencies of less than $10 \mathrm{~Hz}$. At $50 \mathrm{~Hz}$ and above, neuronal firing rate reflects stimulus rate in about $5 \%$ of these cells. Some elements of the dorsal horn are able to encode higher frequencies of vibrotactile pitch in their firing rates, meaning that the dorsal horn may be important in the transmission of this information to the brain in the same way as other classically studied somatosensory nuclei (e.g. dorsal columns, sensory trigeminal nucleus).

\section{B. Periodicity as a possible code for vibrotactile pitch}


Periodicity was studied in DHC discharges to electrical and mechanical stimuli. Overall, the cells preserve the temporal aspects of the stimulus much better in the periodicity of their discharges than in their firing rate.

\section{Phase locking of DHC responses}

Electrically stimulated cells showed a good degree of periodicity in their discharges, especially during 5 and $10 \mathrm{~s}^{-1}$ stimulation. Most cells had vector strengths of greater than .9 , showing that the phase coherence of their responses was very consistent from stimulus to stimulus. At higher stimulation rates, the phase vectors declined in magnitude, but there were many cells that remained well phase locked.

Periodicity in mechanically stimulated $\mathrm{DHC}$ discharges was similar to that observed in the electrically stimulated population. Vector strengths showed a positive correlation with stimulus amplitude, and a negative correlation with stimulus frequency. There were many cells that had high vector strengths at all frequencies of largest amplitude stimulation, indicating that most cells were well phase locked to the sinusoidal stimulation. The phase locking in the DHCs is comparable to that seen in the dorsal column nucleus cells (Ferrington et al., 1987).

\section{Autcorrelograms and power spectra}

Autocorrelograms and power spectra of responses show that most DHCs respond at frequencies that are commensurate with the electrical stimulation 
frequencies, even at 50 and $100 \mathrm{~s}^{-1}$. For most of these cells, the principal frequency component in their autocorrelograms was at the stimulus frequency or one of its harmonics. This means that regardless of how fast the cell was responding (how high its firing rate was), the responses that were produced were in synch with the stimulus.

Analyses of autocorrelograms and power spectra of mechanically stimulated cells showed similar results to the electrical paradigm, although the altering of stimulus amplitude had an effect. The principal frequency components of the power spectra seem to converge to the stimulus frequency or one of its harmonics with increasing amplitude. In general, the $70 \mu \mathrm{m}$ stimulus was not sufficient to drive cells, and the responses increased with greater stimulus amplitude. The power spectra for DHCs at each frequency of stimulation were similar to those obtained with electrical stimulation, when the amplitude of the stimulation was $500 \mu(300 \mu \mathrm{m}$ for $200 \mathrm{~Hz})$.

\section{Analyses of population responses}

The population responses of all sampled cells were examined to determine if their combined responses could encode the pitch of the electrical stimulus. There were no corrections made for conduction distance or velocity, and the power spectra for the responses to all frequencies of electrical stimulation were calculated. The principal frequency components for the power spectra for all four rates of electrical stimulation were near the frequency of the stimulation, and other harmonics of the stimulus frequency were present as well. This suggests that the volley principle from auditory physiology could apply to encoding of vibrotactile pitch in the dorsal horn. The volley 
principle states that each of the neurons responding to a particular stimulus may not respond to every cycle of the stimulus. However, the individual neuronal responses are phase locked to the stimulus. When the responses of all of the responding neurons are summed, all of the stimulus cycles are represented in the aggregate discharge. The population of DHCs whose RFs are being excited by an electrical stimulus may not fire on every cycle of the stimulus, but the information available to higher levels of the CNS provided by that population encodes pitch well in the periodicity of all combined responses.

The population responses for all mechanically stimulated DHCs were not as clear as the population responses from the electrically stimulated cells. This is due to a large low frequency component found in the power spectrum for the responses across all cells. This low frequency component is probably due to the background level of activity across all cells (a DC level introduced by the aperiodic responses of all cells). The autocorrelograms show a periodic discharge riding on a constant background. If this background level is removed, the principal frequency component of the power spectrum corresponds to the stimulus frequency.

\section{Summary}

The results of these experiments show that some DHCs are able to encode vibrotactile pitch, both in the periodicity and rate of their discharges.. On a single neuron basis, these cells appear to be able to encode vibrotactile pitch in the periodicity of their discharge at the frequencies of stimulation used in both the mechanical and electrical stimulation paradigms. However, few neurons in either paradigm had a 
monotonically increasing periodicity and firing rate correlation with increasing frequency of stimulation. This means that very few DHCs are able to follow high frequency ( $>50 \mathrm{~s}^{-}$ ${ }^{1}$ or $>50 \mathrm{~Hz}$ ) stimuli in a 1:1 fashion, such as those reported in the dorsal column nuclei and the thalamus. There were a few neurons that were able to follow electrical stimuli up to $100 \mathrm{~s}^{-1}$, and one that was able to follow $100 \mathrm{~Hz}$ in a greater than $1: 1$ fashion. This shows that there are at least some DHCs that can follow electrical and vibrotactile stimuli in the same way as some other subcortical somatosensory nuclei. The search stimuli in these studies were typically $5 s^{-1}$ or $5 \mathrm{~Hz}$, so cells for study were not selected on the basis of their high frequency following characteristics.

DHCs can receive inputs of several different submodalities, and it is possible that the few cells that responded well to high frequencies of periodic skin stimulation were the ones that were connected to receptors (probably RA and PC) that were able to transduce stimuli of that frequency. Peripheral input was not monitored in these experiments, so the failure of DHCs to respond to high frequency stimulation may simply be a result of the failure of afferents to follow stimuli of a given frequency and amplitude, not the inherent qualities of the DHCs themselves.

There are many questions that are raised by these findings, and much more extensive testing of the coding of vibrotactile pitch is necessary. Study of projection cells (spinothalamic, spinocervical, and postsynaptic dorsal columns) would demonstrate whether or not cells that carry information to the brain in fact are able to encode vibration. Tuning curves of many different combinations of frequency and amplitude would also shed light on how these cells respond, and if they can be classified using the nomenclature described elsewhere (RA, SA, PC). Mechanical tip 
location within the DHC RF may affect the cell's response, as may the diameter of the probe, and pre-indentation. Future studies should focus purely on the response of cells to mechanical stimulation, since that is a natural stimulus for these cells. Factors that affect the responsiveness of these cells could also be examined (e.g. pharmacological agents and descending inhibition).

The DHCs studied in these experiments were able to 1) encode low frequencies of both mechanical and electrical stimulation in their firing rates, but unable to encode higher frequencies, with the exception of a small portion of the cell sample; 2) phase lock to electrical and high amplitude mechanical stimuli, where a negative correlation exists between frequency and vector strength; 3) encode all frequencies of electrical and high amplitude mechanical stimuli in the principal frequency components of their power spectra. This shows that there are some DHCs that respond to periodic stimuli in the same way as cells in other somatosensory nuclei implicated in the transmission of vibrotactile information. There are cells that could be just as important in transmitting vibrotactile pitch information at the level of the spinal dorsal horn level as there are in the dorsal column-medial lemniscal system, which is classically regarded as the important pathway for transmission of this type of information. 


\section{Literature Cited}

1. Bell, J. The structure and function of Pacinian corpuscles: a review. Prog.Neurobiol. 42: 79-128, 1994.

2. Bennett, R. E., Ferrington, D. G., and Rowe, M. Tactile neuron classes within second somatosensory area (SII) of cat cerebral cortex. J.Neurophysiol. 43: 292$309,1980$.

3. Bolanowski, S. J., Gescheider, G. A., and Verrillo, R. T. Hairy skin: psychophysical channels and their physiological substrates. Somatosens.Mot.Res. 11: 279-290, 1994.

4. Bolanowski, S. J., Jr., Gescheider, G. A., Verrillo, R. T., and Checkosky, C. M. Four channels mediate the mechanical aspects of touch. J.Acoust.Soc.Am. 84: 1680-1694, 1988.

5. Brown, A. G. and Iggo, A. A Quantitative Study of Cutaneous Receptors and Afferent Fibres in the Cat and Rabbit. J.Physiol (Lond) 193: 707-733, 1967.

6. Brown, A. G. and Franz, D. N. Responses of spinocervical tract neurones to natural stimulation of identified cutaneous receptors. Exp.Brain Res. 7: 231-249, 1969. 
7. Brown, A. G. and Hayden, R. E. The distribution of cutaneous receptors in the rabbit's hind limb and differential electrical stimulation of their axons. J.Physiol (Lond) 213: 495-506, 1971.

8. Brown, A. G., Koerber, H. R., and Noble, R. An intracellular study of spinocervical tract cell responses to natural stimuli and single hair afferent fibres in cats. J.Physiol (Lond) 382:331-54, 1987.

9. Brown, P. B. Response of Cat Dorsal Horn Cells to Variations of Intensity, Location, and Area of Cutaneous Stimuli. Exp.Neurol. 23: 249-265, 1969.

10. Brown, P. B. and Kanungo, M. Introduction to Microcomputers: A Pulse Interval Timer. J.Electrophysiol.Tech. 12: 81-97, 1985.

11. Brown, P. B., Kanungo, M., and Tapper, D. N. Introduction to Microprocessors: A Universal Interval Histogram Algorithm. J.Electrophysiol.Tech. 12: 249-280, 1985.

12. Burgess, P. R., Petit, D., and Warren, R. M. Receptor types in cat hairy skin supplied by myelinated fibers. J.Neurophysiol. 31: 833-848, 1968.

13. Burgess, P. R., Howe, J. F., Lessler, M. J., and Whitehorn, D. Cutaneous receptors supplied by myelinated fibers in the cat. II. Number of mechanoreceptors excited by a local stimulus. J.Neurophysiol. 37: 1373-1386, 1974. 
14. Burton, H. and Sinclair, R. J. Second somatosensory cortical area in macaque monkeys: 2. Neuronal responses to punctate vibrotactile stimulation of glabrous skin on the hand. Brain Res. 538: 127-135, 1991.

15. Chambers, M. R., Andres, K. H., Duering, M. v., and Iggo, A. The structure and function of the slowly adapting type II mechanoreceptor in hairy skin. Q.J.Exp.Physiol Cogn Med.Sci. 57: 417-445, 1972.

16. Cohen, J. C., Makous, J. C., and Bolanowski, S. J. Under which conditions do the skin and probe decouple during sinusoidal vibrations? Exp.Brain Res. 129: 211217, 1999.

17. Cohen, R. H. and Vierck, C. J., Jr. Population estimates for responses of cutaneous mechanoreceptors to a vertically indenting probe on the glabrous skin of monkeys. Exp.Brain Res. 94: 105-119, 1993.

18. Cohen, R. H. and Vierck, C. J., Jr. Relationships between touch sensations and estimated population responses of peripheral afferent mechanoreceptors. Exp.Brain Res. 94: 120-130, 1993.

19. Connor, K. M., Ferrington, D. G., and Rowe, M. J. Tactile sensory coding during development: signaling capacities of neurons in kitten dorsal column nuclei. J.Neurophysiol. 52: 86-98, 1984. 
20. Connor, K. M., Ferrington, D. G., and Rowe, M. J. Tactile sensory coding during development: signaling capacities of neurons in kitten dorsal column nuclei. J.Neurophysiol. 52: 86-98, 1984.

21. Danziger, N., Remy, P., Pidoux, B., Dormont, D., Samson, Y., Fournier, E., Wall, P. D., and Willer, J. C. A clinical and neurophysiological study of a patient with an extensive transection of the spinal cord sparing only a part of one anterolateral quadrant. Brain 119: 1835-1848, 1996.

22. De Koninck, Y. and Henry, J. L. Peripheral vibration causes an adenosinemediated postsynaptic inhibitory potential in dorsal horn neurons of the cat spinal cord. Neuroscience 50: 435-443, 1992.

23. Donevan, S. D. and Abrahams, V. C. Cat trigeminal neurons innervated from the planum nasale: their medullary location and their responses to mechanical stimulation. Exp.Brain Res. 93: 66-72, 1993.

24. Dubrovsky, B., Davelaar, E., and Garcia-Rill, E. The role of dorsal columns in serial order acts. Exp.Neurol. 33: 93-102, 1971.

25. Edgley, S. A. and Jankowska, E. Information processed by dorsal horn spinocerebellar tract neurones in the cat. J.Physiol (Lond) 397:81-97, 1988. 
26. Ferrington, D. G., Hora, M. O., and Rowe, M. J. Functional maturation of tactile sensory fibers in the kitten. J.Neurophysiol. 52: 74-85, 1984.

27. Ferrington, D. G., Horniblow, S., and Rowe, M. J. Temporal patterning in the responses of gracile and cuneate neurones in the cat to cutaneous vibration. J.Physiol (Lond) 386:277-91, 1987.

28. Ferrington, D. G., Rowe, M. J., and Tarvin, R. P. Integrative processing of vibratory information in cat dorsal column nuclei neurones driven by identified sensory fibres. J.Physiol (Lond) 386:311-331, 1987.

29. Ferrington, D. G., Rowe, M. J., and Tarvin, R. P. Actions of single sensory fibres on cat dorsal column nuclei neurones: vibratory signalling in a one-to-one linkage. J.Physiol (Lond) 386:293-309, 1987.

30. Fitzgerald, M. Cutaneous primary afferent properties in the hind limb of the neonatal rat. J.Physiol (Lond) 383:79-92, 1987.

31. Fitzgerald, M. A physiological study of the prenatal development of cutaneous sensory inputs to dorsal horn cells in the rat. J.Physiol (Lond) 432:473-82, 1991.

32. Ghosh, S., Turman, A. B., Vickery, R. M., and Rowe, M. J. Responses of cat ventroposterolateral thalamic neurons to vibrotactile stimulation of forelimb footpads. Exp.Brain Res. 92: 286-298, 1992. 
33. Gynther, B. D., Vickery, R. M., and Rowe, M. J. Responses of slowly adapting type II afferent fibres in cat hairy skin to vibrotactile stimuli. J.Physiol (Lond) 458:151-69: 151-169, 1992.

34. Gynther, B. D., Vickery, R. M., and Rowe, M. J. Transmission characteristics for the $1: 1$ linkage between slowly adapting type II fibers and their cuneate target neurons in cat. Exp.Brain Res. 105: 67-75, 1995.

35. Herron, P. and Dykes, R. The ventroposterior inferior nucleus in the thalamus of cats: a relay nucleus in the Pacinian pathway to somatosensory cortex. J.Neurophysiol. 56: 1475-1497, 1986.

36. Horch, K. W., Tuckett, R. P., and Burgess, P. R. A key to the classification of cutaneous mechanoreceptors. J.Invest Dermatol. 69: 75-82, 1977.

37. Hulliger, M., Nordh, E., Thelin, A. E., and Vallbo, A. B. The responses of afferent fibres from the glabrous skin of the hand during voluntary finger movements in man. J.Physiol (Lond) 291:233-49, 1979.

38. Hyvarinen, J., Poranen, A., and Jokinen, Y. Influence of attentive behavior on neuronal responses to vibration in primary somatosensory cortex of the monkey. J.Neurophysiol. 43: 870-882, 1980. 
39. Iggo, A. and Ogawa, H. Correlative physiological and morphological studies of rapidly adapting mechanoreceptors in cat's glabrous skin. J.Physiol (Lond) 266: 275-296, 1977.

40. Janig, W. Morphology of rapidly and slowly adapting mechanoreceptors in the hairless skin of the cat's hind foot. Brain Res. 28: 217-231, 1971.

41. Janig, W. The afferent innervation of the central pad of the cat's hind foot. Brain Res. 28: 203-216, 1971.

42. Johansson, R. S. and Vallbo, A. B. Detection of tactile stimuli. Thresholds of afferent units related to psychophysical thresholds in the human hand. J.Physiol (Lond) 297: 405-422, 1979.

43. Johnson, K. O. Reconstruction of population response to a vibratory stimulus in quickly adapting mechanoreceptive afferent fiber population innervating glabrous skin of the monkey. J.Neurophysiol. 37: 48-72, 1974.

44. Kelly, E. F., Trulsson, M., and Folger, S. E. Periodic microstimulation of single mechanoreceptive afferents produces frequency-following responses in human EEG. J.Neurophysiol. 77: 137-144, 1997.

45. Kirkpatrick, D. B. and Kruger, L. Physiological properties of neurons in the principal sensory trigeminal nucleus of the cat. Exp.Neurol. 48: 664-690, 1975. 
46. Knibestol, M. Stimulus-response functions of rapidly adapting mechanoreceptors in human glabrous skin area. J.Physiol (Lond) 232: 427-452, 1973.

47. Knibestol, M. Stimulus-response functions of slowly adapting mechanoreceptors in the human glabrous skin area. J.Physiol (Lond) 245: 63-80, 1975.

48. Lamotte, R. H. and Mountcastle, V. B. Disorders in somesthesis following lesions of parietal lobe. J.Neurophysiol. 42: 400-419, 1979.

49. Lebedev, M. A. and Nelson, R. J. High-frequency vibratory sensitive neurons in monkey primary somatosensory cortex: entrained and nonentrained responses to vibration during the performance of vibratory-cued hand movements. Exp.Brain Res. 111: 313-325, 1996.

50. Libet, B., Alberts, W. W., Wright, E. W., Delattre, L. D., Levin, G., and Feinstein, B. Production of Threshold Levels of Conscious Sensation by Electrical Stimulation of Human Somatosensory Cortex. J.Neurophysiol. 26: 546-578, 1964.

51. Lindblom, U. Properties of Touch Receptors in Distal Glabrous Skin of the Monkey. J.Neurophysiol. 28: 966-985, 1965.

52. Lloyd, D. M., Bolanowski, S. J., Jr., Howard, L., and McGlone, F. Mechanisms of attention in touch. Somatosens.Mot.Res. 16: 3-10, 1999. 
53. Makous, J. C., Friedman, R. M., and Vierck, C. J., Jr. Effects of a dorsal column lesion on temporal processing within the somatosensory system of primates. Exp.Brain Res. 112: 253-267, 1996.

54. Merzenich, M. M. and Harrington, T. H. The sense of flutter-vibration evoked by stimulation of the hairy skin of primates: comparison of human sensory capacity with the responses of mechanoreceptive afferents innervating the hairy skin of monkeys. Exp.Brain Res. 9: 236-260, 1969.

55. Mosso, J. A. and Kruger, L. Receptor categories represented in spinal trigeminal nucleus caudalis. J.Neurophysiol. 36: 472-488, 1973.

56. Mountcastle, V. B., Poggio, G. F., and Werner, G. The Relation of Thalamic Cell Response to Peripheral Stimuli Varied Over an Intensive Continuum. J.Neurophysiol. 26: 807-834, 1964.

57. Mountcastle, V. B., Talbot, W. H., Darian-Smith, I., and Kornhuber, H. H. Neural basis of the sense of flutter-vibration. Science 155: 597-600, 1967.

58. Mountcastle, V. B., Talbot, W. H., Sakata, H., and Hyvarinen, J. Cortical neuronal mechanisms in flutter-vibration studied in unanesthetized monkeys. Neuronal periodicity and frequency discrimination. J.Neurophysiol. 32: 452-484, 1969. 
59. Mountcastle, V. B. Neural mechanisms in somesthesia. In Mountcastle, V. B. ed. Medical Physiology Vol. I. St. Louis, C.V. Mosby Co. 1974, 307-347.

60. Mountcastle, V. B., Steinmetz, M. A., and Romo, R. Frequency discrimination in the sense of flutter: psychophysical measurements correlated with postcentral events in behaving monkeys. J.Neurosci. 10: 3032-3044, 1990.

61. Mountcastle, V. B., Steinmetz, M. A., and Romo, R. Cortical neuronal periodicities and frequency discrimination in the sense of flutter. Cold Spring Harb.Symp.Quant.Biol. 55:861-72, 1990.

62. Mountcastle, V. B. Temporal order determinants in a somesthetic frequency discrimination: sequential order coding. Ann.N.Y.Acad.Sci. 682:150-70, 1993.

63. Murray, G. M., Taub, D. R., Mackie, P. D., Zhang, H. Q., Ghosh, S., and Rowe, M. J. The effects of neonatal median nerve injury on the responsiveness of tactile neurones within the cuneate nucleus of the cat. J.Physiol (Lond) 505: 759-768, 1997.

64. Nathan, P. W., Smith, M. C., and Cook, A. W. Sensory effects in man of lesions of the posterior columns and of some other afferent pathways. Brain 109: 1003-1041, 1986. 
65. Nelson, R. J., Smith, B. N., and Douglas, V. D. Relationships between sensory responsiveness and premovement activity of quickly adapting neurons in areas $3 \mathrm{~b}$ and 1 of monkey primary somatosensory cortex. Exp.Brain Res. 84: 75-90, 1991.

66. Noordenobos, W. and Wall, P. D. Diverse sensory functions with an almost totally divided spinal cord. A case of spinal cord transection with preservation of part of one anterolateral quadrant. Pain 2: 185-195, 1976.

67. Oswaldo-Cruz, E. and Kidd, C. Functional Properties of Neurons in the Lateral Cervical Nucleus of the Cat. J.Neurophysiol. 27: 1-14, 1964.

68. Poggio, G. F. and Mountcastle, V. B. The Functional Properties of Ventrobasal Thalamic Neurons Studied in Unanesthetized Monkeys. J.Neurophysiol. 26: 775806, 1963.

69. Pubols, B. H., Jr. and Pubols, L. M. Tactile receptor discharge and mechanical properties of glabrous skin. Fed.Proc. 42: 2528-2535, 1983.

70. Romo, R., Hernandez, A., Zainos, A., and Salinas, E. Somatosensory discrimination based on cortical microstimulation. Nature 392: 387-390, 1998.

71. Romo, R., Brody, C. D., Hernandez, A., and Lemus, L. Neuronal correlates of parametric working memory in the prefrontal cortex. Nature 399: 470-473, 1999. 
72. Rowe, M. J., Turman, A. B., Murray, G. M., and Zhang, H. Q. Parallel organization of somatosensory cortical areas I and II for tactile processing.

Clin.Exp.Pharmacol.Physiol 23: 931-938, 1996.

73. Salter, M. W. and Henry, J. L. Physiological characteristics of responses of wide dynamic range spinal neurones to cutaneously applied vibration in the cat. Brain Res. 507: 69-84, 1990.

74. Talbot, W. H., Darian-Smith, I., Kornhuber, H. H., and Mountcastle, V. B. The sense of flutter-vibration: comparison of the human capacity with response patterns of mechanoreceptive afferents from the monkey hand. J.Neurophysiol. 31: 301-334, 1968.

75. Tapper, D. N., Galera-Garcia, C., and Brown, P. B. Sinusoidal mechanical stimulation of the tactile pad receptor: tuning curves. Brain Res. 36: 223-227, 1972.

76. Tapper, D. N., Brown, P. B., and Moraff, H. Functional organization of the cat's dorsal horn: connectivity of myelinated fiber systems of hairy skin. J.Neurophysiol. 36: 817-826, 1973.

77. Tommerdahl, M., Whitsel, B. L., Vierck, C. J., Jr., Favorov, O., Juliano, S., Cooper, B., Metz, C., and Nakhle, B. Effects of spinal dorsal column transection on the response of monkey anterior parietal cortex to repetitive skin stimulation. Cereb.Cortex 6: 131-155, 1996. 
78. Tommerdahl, M., Delemos, K. A., Favorov, O. V., Metz, C. B., Vierck, C. J., Jr., and Whitsel, B. L. Response of anterior parietal cortex to different modes of samesite skin stimulation. J.Neurophysiol. 80: 3272-3283, 1998.

79. Tommerdahl, M. Responses of contralateral SI and SII in cat to same-site cutaneous flutter versus vibration. J.Neurophysiol. 82: 1982-1992, 1999.

80. Tommerdahl, M. Response of anterior parietal cortex to cutaneous flutter versus vibration. J.Neurophysiol. 82: 16-33, 1999.

81. Torebjork, H. E. and Ochoa, J. L. Specific sensations evoked by activity in single identified sensory units in man. Acta Physiol Scand. 110: 445-447, 1980.

82. Torebjork, H. E., Schady, W., and Ochoa, J. Sensory correlates of somatic afferent fibre activation. Hum.Neurobiol. 3: 15-20, 1984.

83. Torebjork, H. E. Intraneural microstimulation in man. Its relation to specificity of tactile sensations. Brain. 110: 1509-1529, 1987.

84. Turman, A. B., Morley, J. W., Zhang, H. Q., and Rowe, M. J. Parallel processing of tactile information in cat cerebral cortex: effect of reversible inactivation of SII on SI responses. J.Neurophysiol. 73: 1063-1075, 1995. 
85. Vallbo, A. B. and Hagbarth, K. E. Mechnoreceptor activity recorded from human peripheral nerves. Electroencephalogr.Clin.Neurophysiol. 25: 407, 1968.

86. Vallbo, A. B. and Johansson, R. S. Properties of cutaneous mechanoreceptors in the human hand related to touch sensation. Hum.Neurobiol. 3: 3-14, 1984.

87. Vickery, R. M., Gynther, B. D., and Rowe, M. J. Synaptic transmission between single slowly adapting type I fibres and their cuneate target neurones in cat. J.Physiol (Lond) 474: 379-392, 1994.

88. Vierck, C. J., Jr. Tactile movement detection and discrimination following dorsal column lesions in monkeys. Exp.Brain Res. 20: 331-346, 1974.

89. Vierck, C. J., Jr., Cohen, R. H., and Cooper, B. Y. Effects of spinal lesions on temporal resolution of cutaneous sensations. Somatosens.Res. 3: 45-56, 1985.

90. Werner, G. and Mountcastle, V. B. Neural Activity in Mechanoreceptive Cutaneous Afferents: Stimulus-Response Relations, Weber Functions, and Information Transmission. J.Neurophysiol. 27: 396, 1965.

91. Whitehorn, D. and Burgess, P. R. Changes in polarization of central branches of myelinated mechanoreceptor and nociceptor fibers during noxious and innocuous stimulation of the skin. J.Neurophysiol. 36: 226-237, 1973. 
92. Whitsel, B. L., Favorov, O., Delemos, K. A., Lee, C., Tommerdahl, M., Essick, G. K., and Nakhle, B. SI neuron response variability is stimulus tuned and NMDA receptor dependent. J.Neurophysiol. 81: 2988-3006, 1999.

93. Wu, G., Ekedahl, R., Stark, B., Carlstedt, T., Nilsson, B., and Hallin, R. G. Clustering of Pacinian corpuscle afferent fibres in the human median nerve. Exp.Brain Res. 126: 399-409, 1999.

94. Zhang, H. Q., Murray, G. M., Turman, A. B., Mackie, P. D., Coleman, G. T., and Rowe, M. J. Parallel processing in cerebral cortex of the marmoset monkey: effect of reversible SI inactivation on tactile responses in SII. J.Neurophysiol. 76: 36333655, 1996. 


\section{CURRICULUM VITAE \\ Jeffrey J. Lawson}

November 14, 2000

PRESENT POSITION

TELEPHONE:

PERSONAL DATA:

EDUCATION:

AWARDS AND

FELLOWSHIPS:

SOCIETIES:

TEACHING

EXPERIENCE:
Graduate Student

Department of Physiology

West Virginia University Medical Center

Morgantown, WV 26506

(304) 293-3524

BORN: $5 / 8 / 70$

CITIZENSHIP: U.S.A.

FAMILY: $\quad$ Single

HOME

ADDRESS: $\quad 807$ Alpine St. \#9

Morgantown, WV 26505

(304) 599-2527

B.A., Biology, Washington and Jefferson College

Washington and Jefferson Presidential Scholarship Recipient, 1988 - 1992

Society for Neuroscience

Human Physiology for pre-professional students, 1996-Present

Human Physiology, neuroscience block, 1996-Present

\section{RESEARCH:}

My primary interest as a student has been the neurobiology of spinal cord neurons processing information from low threshold cutaneous mechanoreceptors. These cells are believed to play an important role in light touch sensation. 
My dissertation project involved the study of response characteristics of spinal dorsal horn neurons to electrical and mechanical skin stimuli. This series of studies has shown that there are several ways in which these neurons may be able to encode information about the frequency and amplitude of applied electrical and vibratory skin stimuli. I have focused on learning quantitative analytical techniques to investigate the information contained in trains of action potentials in sensory neurons.

I plan to continue my research career by looking at the relationship between the behavior of neurons in sensory pathways and perceptual events that reach consciousness.

\section{PUBLICATIONS}

\section{A. ABSTRACTS}

1. Lawson, J, R Millecchia, J L Culberson, S Stephens, P B Brown: Correlation of dermatomelets and dorsal horn cell RFs at the level of entry of dorsal rootlets. Soc. Neurosci. Abstr. 22:99, 1996.

2. Brown, PB, R Millecchia, J Lawson, J L Culberson, S Stephens. A model of lamina III - IV cell receptive fields. Soc. Neurosci. Abstr. 22:98, 1996.

3. Stephens, S, R Millecchia, J L Culberson, J Lawson, W Gladfelter, P B Brown: Rostrocaudal distributions of cord dorsum potentials and observed and modeled single-unit responses. Soc. Neurosci. Abstr. 22:99, 1996.

4. Millecchia, R, S Stephens, J Lawson, J Culberson, PB Brown, P Harton and L Hicks: Reconstruction of 2-D somatotrophy from 1-D CDPs. Soc. Neurosci. Abstr. 23:2342, 1997.

5. Stephens, S, R Millecchia, J Culberson, J Lawson, P Harton, M Fields, P B Brown: Unmasking of dorsal horn receptive fields by local anesthesia. Soc. Neurosci. Abstr. 23:2342, 1997.

6. Miller, M, M Field, I Goodman, P Harton, L Hicks, A Johnson, J Lawson, R Millecchia, V Odom, S Stephens, P Brown: Effects of location and orientation on acuity in 2 point discrimination in humans and cats: Relations to properties of somatosensory system. Soc. Neurosci. Abst. 24:435, 1998.

7. Lawson, J, R Millecchia, P Harton, J Culberson, M Miller, S Stephens, P Brown: Comparison of preand postsynaptic somatotopy in cat at the level of the dorsal horn. Soc. Neurosci. Abst. 24: 2079, 1998.

8. Millecchia, R, J Lawson, J Culberson, P Harton, I Goodman, PB Brown: A signal detection model of two point discrimination. Soc. Neurosci. Abstr. 25:402; 1999.

9. Lawson, J. R Millecchia, S Stephens, P Harton, J Culberson, PB Brown: Comparison of primary afferent projections predicted from cord dorsum potentials with HRF-filled axons. Soc. Neurosci. Abstr. 25:402;1999.

10. Brown, PB, R Millecchia, J Lawson, J Culberson, P Harton: Spatial convergence and divergence between cutaneous afferent axons and dorsal horn cells are not constant. Soc. Neurosci. Abstr. 25:402;1999.

\section{B. PEER REVIEWED PAPERS}


1. Brown, PB, R Millecchia, J Lawson, S Stephens, P Harton JL Culberson: Dorsal horn spatial representation of simple cutaneous stimuli. J. Neurophysiol. 79:983-998,1998.

\section{IN PRESS:}

1. PB Brown, P Harton, R Millecchia,J Lawson, Tisana Kunjara-Na-Ayudhya, S Tephens, M Miller, L Hicks, J Culberson: Spatial convergence and divergence between cutaneous afferent axons and dorsal horn cells are not constant. (accepted by J. Comp. Neurol.)

\section{IN PREPARATION:}

1. Millecchia, RJ, JL Lawson, S Stephens, P Harton, J Culberson, and PB Brown. Primary afferent projections to dorsal horn reconstructed from cord dorsum potentials: test of a quantitative developmental model. (to be submitted to J. Neurosci.)

2. Lawson, J, JL Culberson, R Millecchia, S Stephens, PB Brown. Embryonic dorsal root afflux and local mediolateral sorting establish adult dorsal horn presynaptic somatotopy. (to be submitted to $\mathrm{J}$. Neurosci.).

4. Brown, PB, R Millecchia, JL Culberson, S Stephens, J Lawson, P Harton: Quantitative analysis of dorsal horn cell receptive field changes in cats with spared $S_{1}$ (to be submitted to J. Neurosci.).

5. Brown, PB, R Millecchia, J Lawson, JL Culberson, S Stephens, P Harton: Quantitative analysis of dorsal horn cell receptive field changes in cats with crushed regenerated sural nerve and cut, nonregenerated $\mathrm{PFCN}_{3}$ (to be submitted to $\underline{\mathrm{J} \text {. Neurosci.) }}$ 